Division of Geological \& Geophysical Surveys

RAW-DATA FILE 2005-4

Version 1.1

\title{
GEOCHEMICAL DATA FROM REANALYSIS OF STREAM-SEDIMENT SAMPLES \\ COLLECTED IN 1982 FROM THE LIVENGOOD AREA, TOLOVANA MINING DISTRICT, ALASKA
}

by

D.J. Szumigala, C.C. Puchner, and R.E. Myers

$\$ 5.00$

December 2005

THIS REPORT HAS NOT BEEN REVIEWED FOR TECHNICAL CONTENT OR FOR CONFORMITY TO THE EDITORIAL STANDARDS OF DGGS

Released by

STATE OF ALASKA

DEPARTMENT OF NATURAL RESOURCES

Division of Geological \& Geophysical Surveys 3354 College Road

Fairbanks, Alaska 99709-3707 


\section{CONTENTS}

PAGE

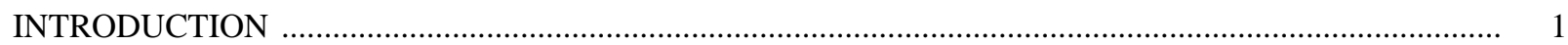

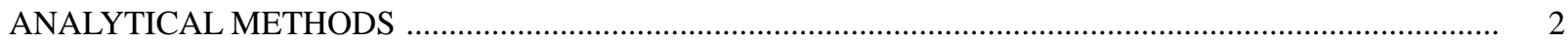

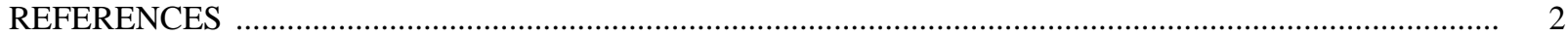

\section{TABLES}

TABLE 1. LOCATION AND NEW TRACE ELEMENT GEOCHEMICAL RESULTS FOR 902 STREAM-SEDIMENT SAMPLES COLLECTED IN THE LIVENGOOD AREA, LIVENGOOD

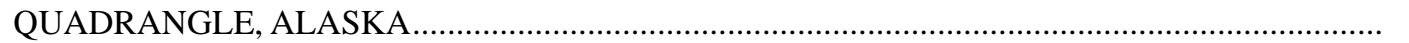

TABLE 2. DETECTION LIMITS FOR TRACE-ELEMENT GEOCHEMICAL ANALYSES 3 45

Note: This report (including all analytical data, tables, and map sheets) is available in digital format from the DGGS web site (http://wwwdggs.dnr.state.ak.us ) at no charge. The digital data are available as PDF files and Excel spreadsheets. 


\title{
GEOCHEMICAL DATA FROM REANALYSIS OF STREAM-SEDIMENT SAMPLES COLLECTED IN 1982 FROM THE LIVENGOOD AREA, TOLOVANA MINING DISTRICT, ALASKA
}

\author{
by \\ D.J. SZUMIGALA ${ }^{1}$, C.C. PUCHNER ${ }^{2}$, AND R.E. MYERS ${ }^{3}$ \\ ${ }^{1}$ Alaska Division of Geological \& Geophysical Surveys, 3354 College Rd, Fairbanks, AK 99709-3707 \\ ${ }^{2}$ Hesperus Geoscience, P.O. Box 74848, Fairbanks, AK 99707-4848 \\ ${ }^{3}$ AngloGold Ashanti North America Inc., 7400 East Orchard Rd, Suite 350, Greenwood Village, CO 80111
}

\section{INTRODUCTION}

The geochemical analyses tabulated in this report result from reanalysis of archived stream-sediment pulps. The stream-sediment samples were originally collected in 1982 as part of a geological and mineral investigation of the Livengood mining district by DGGS. The original geochemical data set included 1,597 stream-sediment samples, 297 pan-concentrate samples, and 305 rock samples from the Livengood B-3, B-4, C-3, and C-4 quadrangles (Albanese, 1983).

The new data set is the result of an agreement between DGGS and AngloGold Ashanti North America Inc. (AngloGold), a global gold company with 22 operations on four continents. The agreement allowed AngloGold to reanalyze selected DGGS geochemical samples collected in the Livengood area during 1982. The agreement gave AngloGold 5 months during 2004 to analyze the samples, after which AngloGold gave DGGS all analytical results from their (and third-party company) work and returned any unused sample pulps. The mission of this project is aligned with those of the Alaska Airborne Geophysical/Geological Mineral Inventory Program, to expand the knowledge base of Alaska's mineral resources and to catalyze private-sector mineral development. This data set complements data previously generated during the 2003 DGGS field investigations over part of the Livengood area.

David Szumigala found the archived sample pulps from the 1982 field study in the DGGS Fairbanks storage area in March 2004. Some pulps were destroyed by the weather during storage, some sample numbers were unreadable, and some of the original samples may not have had enough pulp material to archive. AngloGold Ashanti contract geologists, as part of an agreement with DGGS, selected 952 stream-sediment sample pulps (60 percent of the original stream-sediment sample set) as suitable for reanalysis. Fifty (50) sample pulps were rejected for analysis because of insufficient weight. AngloGold Ashanti geologists submitted the 902 sample pulps, including 40 additional blank and non-reference standards, to ALS Chemex for geochemical analysis by mid-year 2004. The geochemical analyses were given to DGGS in late 2004 by AngloGold Ashanti as a Microsoft Excel spreadsheet containing: a worksheet with compiled new analytical results including digitized sample coordinates; a worksheet containing analytical results for blank and standard reference samples included in the samples analyzed by ALS Chemex; and 4 worksheets of the assay certificate files as downloaded from the ALS Chemex client-accessible secured Website.

Location data were determined by digitizing sample locations as shown on location maps in Albanese (1983). Location data (in UTM coordinates with a Clark 1866, NAD27, UTM zone 6 projection) and analytical results for each sample are tabulated in Table 1.

AngloGold Ashanti provided funding for the geochemical analyses and digitizing of sample locations. Publication of this data is funded through State of Alaska General Funds. 


\begin{abstract}
ANALYTICAL METHODS
ALS Chemex in Vancouver, B.C., Canada performed all geochemical analyses tabulated in this report. Streamsediment sample pulps were used from the DGGS storage area in Fairbanks. Stream sediment samples were collected in 1982 with a steel shovel from the finer sandy portion of active channels or deepest most active part of dry creek beds. Organic-rich material was avoided. Float rock and stream characteristics were noted and recorded at each station. The pulps were prepared at the DGGS Geochemical Laboratory in 1982 by air-drying before screening at minus-80 mesh. Blank and non-reference standards were included at irregular intervals in the samples submitted to ALS Chemex in 2004. This report does not show the results from those control samples, but all control samples had analytical results within acceptable limits. Geochemical samples were analyzed for gold using atomic absorption spectrometry (AAS) following a fire assay fusion. Samples (0.50 grams) submitted for mercury analyses were digested with aqua regia for at least one hour in a hot water bath. After cooling, the resulting solution was diluted to $12.5 \mathrm{ml}$ with demineralized water and mixed. A portion of the sample was treated with stannous chloride to reduce the mercury. The resulting mercury was volatilized by argon-purging and measured by atomic absorption spectrometry (AAS). All other geochemical analyses (47 elements) were performed by a combination of inductively coupled plasma-atomic emission spectroscopy (ICP-AES) and inductively coupled plasma - mass spectrometry (ICP-MS) methods after $\mathrm{HF}-\mathrm{HNO}_{3}-\mathrm{HClO}_{4}$ acid digestion and a $\mathrm{HCl}$ leach. A prepared sample (0.500 gram) was digested with perchloric, nitric and hydrofluoric acids to dryness. The residue was taken up in nitric and hydrochloric acids and diluted to a final volume with deionized water. The resulting solution was analyzed by inductively coupled plasma-atomic emission spectrometry (ICP-AES). Following this analysis, the results were reviewed to ensure that base metal concentrations were less than $1 \%$, with the exception of silver, bismuth, and tungsten which have upper analytical limits of 100, 500, and $1000 \mathrm{ppm}$, respectively. Samples that meet these criteria were then diluted and analyzed by inductively coupled plasma - mass spectrometry (ICP-MS). Results were corrected for spectral interelement interferences. Samples that fail to meet the upper concentration limits would be analyzed by other methods (all results in this report were below the upper detection limits for any given element). This method of digestion is possibly incomplete for certain elements (Ba, $\mathrm{Cr}, \mathrm{Sn}, \mathrm{Ta}, \mathrm{Ti}, \mathrm{W}$, and $\mathrm{Zr}$ ) and may result in lower analytical results. Analytical detection limits are tabulated in Table 2.
\end{abstract}

\title{
REFERENCES
}

Albanese, M.D., 1983, Geochemical reconnaissance of the Livengood B-3, B-4, C-3, and C-4 Quadrangles, Alaska: summary of data on stream-sediment, pan-concentrate, and rock samples: Alaska Division of Geological \& Geophysical Surveys Report of Investigation 83-1, 55 p., 4 sheets, scale 1:63,360. 
Table 1. Location and New Trace Element Geochemical Results for 902 Stream-Sediment Samples collected in the Livengood Area, Livengood Quadrangle, Alaska IS = Insufficient Sample for Analysis

\begin{tabular}{|c|c|c|c|c|c|c|c|c|c|c|c|c|c|c|c|c|c|c|c|c|c|c|c|c|c|c|c|}
\hline MPLE & TM E & TM N & $\begin{array}{c}\mathrm{Au} \\
\mathrm{ppm}\end{array}$ & $\begin{array}{c}\mathrm{Ag} \\
\mathrm{ppm}\end{array}$ & $\begin{array}{l}\text { Al } \\
\%\end{array}$ & $\begin{array}{l}\text { As } \\
\text { ppm }\end{array}$ & $\begin{array}{l}\mathrm{Ba}^{*} \\
\mathrm{ppm}\end{array}$ & \begin{tabular}{|c|}
$\mathrm{Be}$ \\
$\mathrm{ppm}$ \\
\end{tabular} & $\begin{array}{c}\mathrm{Bi} \\
\mathrm{ppm}\end{array}$ & $\begin{array}{c}\mathrm{Ca} \\
\% \\
\end{array}$ & \begin{tabular}{|c|}
$\mathrm{Cd}$ \\
$\mathrm{ppm}$ \\
\end{tabular} & $\begin{array}{r}\mathrm{Ce} \\
\mathrm{ppm}\end{array}$ & $\begin{array}{l}\text { Co } \\
\text { ppm }\end{array}$ & $\begin{array}{l}\mathrm{Cr}^{*} \\
\mathrm{ppm}\end{array}$ & $\begin{array}{c}\text { Cs } \\
\text { ppm }\end{array}$ & $\begin{array}{r}\mathrm{Cu} \\
\mathrm{ppm}\end{array}$ & $\begin{array}{c}\mathrm{Fe} \\
\% \\
\end{array}$ & $\begin{array}{c}\mathrm{Ga} \\
\mathrm{ppm}\end{array}$ & $\begin{array}{c}\mathrm{Ge} \\
\mathrm{ppm}\end{array}$ & $\begin{array}{c}\mathrm{Hf} \\
\mathrm{ppm} \\
\end{array}$ & $\begin{array}{l}\mathrm{Hg} \\
\mathrm{ppm}\end{array}$ & $\begin{array}{c}\text { In } \\
\text { ppm }\end{array}$ & $\begin{array}{l}\mathrm{K} \\
\% \\
\end{array}$ & $\begin{array}{r}\mathrm{La} \\
\mathrm{ppm}\end{array}$ & $\begin{array}{c}\mathrm{Li} \\
\mathrm{ppm} \\
\end{array}$ & $\begin{array}{c}\mathrm{Mg} \\
\% \\
\end{array}$ & $\begin{array}{l}\mathrm{Mn} \\
\mathrm{ppm}\end{array}$ \\
\hline 3 & 1674 & 265559 & & 0.13 & 5.31 & 6.0 & 750 & 1.07 & 0.12 & & \begin{tabular}{|l}
0.74 \\
\end{tabular} & & 17.1 & & 2.21 & 32.4 & 3.11 & 11.80 & 0.13 & \begin{tabular}{|c|}
1.4 \\
\end{tabular} & 0.16 & 0.042 & \begin{tabular}{|l|}
0.94 \\
\end{tabular} & 27.2 & 22.8 & 1.28 & 716 \\
\hline 5 & & & & & \begin{tabular}{|l}
4.99 \\
\end{tabular} & 13.6 & 10 & 20 & & 19 & \begin{tabular}{|l|}
0.95 \\
\end{tabular} & 45.9 & 27. & & 2.1 & 87.5 & 4.93 & 14.25 & 0.15 & 2.3 & 0.78 & 0.062 & \begin{tabular}{|l}
0.98 \\
\end{tabular} & 19.4 & 8.3 & \begin{tabular}{|l|}
1.30 \\
\end{tabular} & 1195 \\
\hline 1 & & & 013 & 28 & 5.39 & 11.8 & 50 & 23 & 0.13 & 1.22 & \begin{tabular}{|l|}
1.08 \\
\end{tabular} & 52.7 & 27.9 & & 2.04 & 69.4 & 5.60 & 14.45 & 0.13 & 2.7 & 0.22 & 0.058 & \begin{tabular}{|l|}
0.97 \\
\end{tabular} & 22.8 & 8.4 & 1.23 & 1380 \\
\hline & & & & 30 & & & 20 & & & & & & & & & & & & & 24 & & & \begin{tabular}{|l}
0.85 \\
\end{tabular} & & & 1.24 & \\
\hline 9 & & & .012 & 0.14 & 4.82 & 21.4 & 720 & .01 & .14 & 1.89 & \begin{tabular}{|l|}
0.61 \\
\end{tabular} & 62.5 & 17.7 & 84 & 1.79 & 26.9 & 4.40 & 11.00 & 0.13 & 1.7 & 0.20 & 0.041 & \begin{tabular}{|l|}
0.91 \\
\end{tabular} & 29.5 & 19.4 & 1.00 & 1180 \\
\hline$\overline{1}$ & & & 015 & 0.16 & 5.41 & 10.4 & 980 & 30 & 0.11 & 1.34 & 1.26 & 60.3 & 25.6 & 108 & 1.89 & 54.6 & 5.13 & 13.95 & 0.17 & 2.5 & 0.14 & 0.056 & 0.91 & 27.6 & \begin{tabular}{|l|l|}
18. \\
\end{tabular} & 1.17 & 1115 \\
\hline & & & 05 & & \begin{tabular}{|l|l}
6.01 \\
\end{tabular} & 10.0 & 770 & & & & & & 8.7 & & 2.82 & 22.4 & 268 & 14.30 & 0.11 & 1.7 & $\overline{0.44}$ & & & 330 & & 0.81 & 320 \\
\hline 4 & & & 0.015 & 0.17 & 6.28 & 9.2 & 800 & 1.12 & 0.16 & 1.54 & 40 & 62.6 & 12.5 & 8 & 2.86 & 21.0 & 3.08 & 12.90 & 0.10 & 1.4 & 0.27 & 0.044 & 1.26 & 29.2 & 27.1 & 1.06 & 001 \\
\hline & & & .005 & 13 & 6.07 & 8.5 & 840 & 1.14 & 0.13 & 1.88 & 0.24 & 74.7 & 12.5 & & 2.27 & 25.8 & 3.11 & 13.05 & 0.13 & 1.6 & 1.95 & 0.042 & 1.25 & 35.6 & 22.9 & 1.08 & 562 \\
\hline & & & 006 & 07 & 6.32 & 3.0 & 850 & 1.13 & 0.12 & 1.86 & \begin{tabular}{|l}
0.23 \\
\end{tabular} & 72.1 & 11.8 & & 2.20 & 20.4 & 3.09 & 12.85 & 0.15 & 1.6 & 0.86 & 0.042 & 1.25 & 34.0 & 22.8 & 1.09 & 55 \\
\hline 5 & & & 0.008 & 0.16 & \begin{tabular}{|l|l|}
6.18 \\
\end{tabular} & 7.9 & 800 & .12 & 0.14 & 1.50 & \begin{tabular}{|l|l}
0.37 \\
\end{tabular} & 61.4 & 12.2 & 8 & 2.76 & 20.9 & 3.01 & 13.30 & 0.11 & 1.4 & 0.29 & 0.043 & \begin{tabular}{|l}
1.28 \\
\end{tabular} & 28.4 & 27.4 & 1.04 & 662 \\
\hline & & & $\overline{006}$ & 84 & 5.60 & 31.9 & 740 & 01 & 7 & 0.73 & \begin{tabular}{|l|}
0.41 \\
\end{tabular} & $0 . c^{-1}$ & 7.2 & & 5.79 & 06.0 & 3.19 & 12.50 & 0.14 & 1. & 9.07 & 0.072 & \begin{tabular}{|l|l}
1.76 \\
\end{tabular} & 44.9 & 16.7 & 0.80 & 247 \\
\hline & & & .007 & 54 & 82 & 1.0 & 740 & 37 & 2.69 & 1.10 & .42 & $\overline{0.4}$ & 25. & & 10.60 & 92.3 & 5.23 & 15.00 & 0.17 & 1. & 0.54 & .410 & 1.47 & 8.0 & 99.2 & 1.06 & 710 \\
\hline & & & .009 & 0.53 & 7.13 & 33.0 & 770 & 1.50 & 3.40 & 0.66 & \begin{tabular}{|l}
1.27 \\
\end{tabular} & 84.4 & 20.1 & 106 & 9.30 & 107.5 & 7.39 & 15.70 & 0.18 & 1.6 & 1.88 & 0.510 & 1.70 & 41.3 & 43.9 & 1.16 & 1770 \\
\hline & & & 007 & 0.39 & 6.16 & 2.6 & 720 & & & 1.22 & \begin{tabular}{|l|}
0.71 \\
\end{tabular} & & 17.8 & & 5.93 & 64.5 & 3.50 & 13.10 & 0.13 & 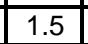 & 0.85 & .016 & \begin{tabular}{|l}
1.29 \\
\end{tabular} & 29.7 & 30.2 & 0.96 & 722 \\
\hline & & & 0.017 & 0.95 & 5.87 & 54.6 & 620 & $\overline{08}$ & 1.07 & 1.07 & \begin{tabular}{|l|}
2.84 \\
\end{tabular} & $\overline{0.8}$ & 19.8 & 96 & 12.25 & 70.8 & 4.05 & 2.30 & 0.13 & 1.3 & 3.47 & 0.160 & 1.29 & 23.2 & 33.6 & 1.04 & 1600 \\
\hline & & & $\overline{006}$ & 43 & 7.70 & 97.0 & 830 & 34 & 2.79 & 0.40 & 61 & 3.6 & 16. & 16 & 5.45 & 18. & 5.29 & 8.50 & 0.18 & 2.6 & 2.73 & .128 & 2.30 & 25.8 & 45.3 & 1.43 & 962 \\
\hline & & & 005 & $\overline{15}$ & 6.45 & & 50 & & & 32 & & & & & & & & 4.00 & 0.14 & & 44 & 049 & 1.23 & & 26.1 & $\overline{1.0}$ & 93 \\
\hline & & & 005 & 0.14 & 6.48 & 10.4 & 820 & 1.2 & 0.27 & 1.35 & 0.33 & 71.9 & 15. & 9 & 3.02 & 25.3 & 3.48 & 14.20 & 0.14 & 1.7 & 0.84 & 049 & 1.25 & 33.8 & 27.2 & 1.06 & 653 \\
\hline & & & $\overline{00 !}$ & & .04 & 8.9 & 770 & & & 1.39 & & $\overline{6}$ & 10 & & 2.6 & 20. & 3.02 & 12.75 & 0.14 & 1. & 0.84 & .044 & 1.21 & 29.6 & 23.2 & 0.96 & 411 \\
\hline & & & .005 & 15 & \begin{tabular}{|l}
6.56 \\
\end{tabular} & 10.0 & 860 & & 0.47 & 1.48 & \begin{tabular}{|l|}
0.42 \\
\end{tabular} & 6 & 25. & 8 & 2.81 & 21. & 3.54 & 3.95 & 0.17 & & & 042 & 34 & 9.9 & 6.3 & 1.02 & 1420 \\
\hline & & & .005 & 0.15 & 6.60 & 10.2 & 900 & 1.30 & 0.64 & 1.46 & 0.30 & 67.2 & 13. & 8 & 2.90 & 27.2 & 3.53 & 15.05 & 0.13 & 1.6 & 0.54 & .048 & 1.36 & 31.6 & 27.2 & 1.05 & 671 \\
\hline & & & .005 & 16 & & 9.7 & 710 & & & .74 & & 56. & 14. & & 5.1 .5 & 22. & 3.00 & 5.85 & 0.11 & 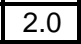 & 0.08 & .052 & \begin{tabular}{|l|l|} 
\\
\end{tabular} & 26.2 & 27.6 & 0.78 & 714 \\
\hline & & & 097 & & & & 750 & & & & & & & & & & & & & & & & & 27 & 24.2 & & 27 \\
\hline & & & .006 & 0.10 & \begin{tabular}{|l|l|}
6.01 \\
\end{tabular} & 9 & 760 & & 0.1 & .20 & .28 & 67. & 10. & & 2.4 & 20. & 2.99 & 13.95 & 0.1 & 2. & 0.06 & 0.044 & \begin{tabular}{|l}
1.13 \\
\end{tabular} & 31.9 & 24.7 & 0.85 & 433 \\
\hline & & & .005 & 20 & & 11.9 & 71 & & & 84 & & & 24 & & & 39 & 3.35 & 3.40 & 0.1 & & 1.1 & 043 & 1.19 & 27.0 & 4.1 & 0.80 & 1655 \\
\hline & & & 005 & 11 & 5.99 & $\begin{array}{l}8.3 \\
\end{array}$ & 850 & 1.28 & & .70 & 0.30 & 69.3 & & & 2.21 & 20.8 & 2.84 & 3.25 & 0. & 1 & 0.12 & & 1.24 & 32.7 & 4.2 & 0.98 & 532 \\
\hline & & & .005 & & $\sqrt{6}$ & 8. & 870 & & & 1.80 & & 78. & & & 2.2 & 20. & 3.02 & 3.55 & 0 & 2. & 0.13 & 04 & 1.24 & 37.1 & 24.5 & 0.98 & 537 \\
\hline & & & & 15 & 6. & 10.0 & 910 & & & 1.04 & & & & & & & 3.1 & 4.05 & & 2. & & & & 39 & 27.4 & 0.96 & 622 \\
\hline & & & & & & & 920 & & & & & & & & & & $\sqrt{31}$ & & & & 7 & & 1.30 & 36 & & 1.00 & 596 \\
\hline & & & 012 & 10 & \begin{tabular}{|l|l|}
6.08 \\
\end{tabular} & 9.7 & 790 & & & 1.58 & & 80.2 & & & 2.19 & 21.8 & 3.16 & 13.40 & 0.15 & 2. & 0.11 & .050 & 1.19 & 37.4 & 24.1 & 0.95 & 785 \\
\hline & & & & & 5.24 & 9 & 570 & & & & & 84 & & & & & 2.51 & 2.80 & & 1. & 0.24 & & 1.34 & 41.8 & 37.3 & 0.72 & 32 \\
\hline & & & .005 & & \begin{tabular}{|l}
6.41 \\
\end{tabular} & 13.8 & 220 & & & & & & & & & 40.4 & 3.30 & 0.60 & & & 251 & & 1.98 & 36.6 & 3 & 0.82 & 674 \\
\hline 97 & & 8 & .021 & 0.12 & \begin{tabular}{|l}
7.61 \\
\end{tabular} & 11.4 & 770 & 1.92 & 0.24 & 0.90 & \begin{tabular}{|l}
0.35 \\
\end{tabular} & 91.9 & 17.9 & 89 & 5.45 & 26.2 & 3.43 & 19.45 & 0.15 & 2.0 & 0.40 & 0.055 & 1.90 & 42.0 & 92.8 & 0.80 & 1005 \\
\hline & & & & & & 8.4 & 860 & & & 1.11 & & & 13.8 & & & & 3.33 & 5.45 & & 2.4 & 0.38 & & 1.58 & 35.2 & 7.7 & 0.90 & 833 \\
\hline & & & 207 & & & 8 & 720 & & & & & & 9. & & & & 2.51 & 4.80 & & 1.7 & 0.20 & .041 & & 27.6 & 6.9 & \begin{tabular}{|l}
0.78 \\
\end{tabular} & 407 \\
\hline & & 215 & 007 & 0.35 & 6.45 & 18.4 & 730 & 1. & 0.3 & 0.60 & 0.3 & 71. & 49. & & 5.5 & & 4.25 & 18.55 & 0.20 & 2.3 & 1.42 & .060 & \begin{tabular}{|l|l}
1.72 \\
\end{tabular} & 32.2 & 18.1 & 0.73 & 3230 \\
\hline & & & & & & & & & & & & & & & & & & & & & 80 & & \begin{tabular}{|l|l|} 
\\
\end{tabular} & 339 & 4 & 0.90 & 7730 \\
\hline & & & .005 & 0.32 & 6.19 & 18 & 630 & 1. & 0. & 0.69 & & 63 & & 95 & 4.28 & 74.8 & 4.34 & 15.05 & 19 & 1.8 & 1.14 & 0.053 & \begin{tabular}{|l|l}
1.68 \\
\end{tabular} & 29.1 & 9.8 & 0.86 & 4650 \\
\hline & & & .005 & \begin{tabular}{|l|}
0.13 \\
\end{tabular} & \begin{tabular}{|l|l|}
6.70 \\
\end{tabular} & 17.6 & 770 & 1. & 0.2 & 0.82 & 0. & 70 & 33.7 & 112 & 4.0 & 40.2 & 4.43 & 16.80 & 0 & 2.0 & 0.66 & 0.055 & 1.71 & 31.7 & 22.8 & \begin{tabular}{|l}
0.93 \\
\end{tabular} & 3490 \\
\hline & & & & & & & 78 & & & & & & & & & & 3.4 & & & 2.4 & 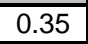 & & 1.37 & 30.2 & 2 & \begin{tabular}{|l}
0.92 \\
\end{tabular} & 1365 \\
\hline & & 727 & $<0.005$ & 0.14 & 5.70 & 11.4 & 690 & 1. & 0.2 & 1.00 & 0.21 & 58. & $\begin{array}{l}12.7 \\
\end{array}$ & 80 & 3.3 & 24.2 & 3.0 & 14.30 & & 1.8 & 0.21 & 0.044 & 1.30 & 27.8 & 24.2 & 0.82 & 800 \\
\hline & & I & 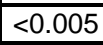 & & 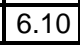 & 6.4 & 890 & & 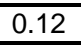 & & & 00.0 & 11.0 & & 2.00 & 15.8 & 3.02 & & & 1.8 & 0.11 & & & 29.1 & & 1.20 & 502 \\
\hline
\end{tabular}


Table 1. Location and New Trace Element Geochemical Results for 902 Stream-Sediment Samples collected in the Livengood Area, Livengood Quadrangle, Alaska IS = Insufficient Sample for Analysis

\begin{tabular}{|c|c|c|c|c|c|c|c|c|c|c|c|c|c|c|c|c|c|c|c|c|c|c|c|c|c|c|}
\hline SAMPLE & $\begin{array}{l}\text { Mo } \\
\mathrm{ppm}\end{array}$ & $\begin{array}{c}\mathrm{Na} \\
\% \\
\end{array}$ & $\begin{array}{l}\mathrm{Nb} \\
\mathrm{ppm}\end{array}$ & $\begin{array}{c}\mathrm{Ni} \\
\mathrm{ppm}\end{array}$ & $\begin{array}{c}\mathrm{P} \\
\mathrm{ppm}\end{array}$ & $\begin{array}{l}\mathrm{Pb} \\
\mathrm{ppm}\end{array}$ & $\begin{array}{l}\mathrm{Rb} \\
\mathrm{ppm}\end{array}$ & $\begin{array}{l}\mathrm{Re} \\
\mathrm{ppm}\end{array}$ & $\begin{array}{l}\mathrm{S} \\
\%\end{array}$ & $\begin{array}{l}\mathrm{Sb} \\
\mathrm{ppm}\end{array}$ & $\begin{array}{l}\text { Se } \\
\mathrm{ppm}\end{array}$ & $\begin{array}{l}S^{\text {Sn }} \\
\mathrm{ppm}\end{array}$ & $\begin{array}{c}\mathrm{Sr} \\
\mathrm{ppm}\end{array}$ & $\begin{array}{l}\mathrm{Ta}^{*} \\
\mathrm{ppm}\end{array}$ & $\begin{array}{c}\text { Te } \\
\text { ppm }\end{array}$ & $\begin{array}{c}\text { Th } \\
\mathrm{ppm}\end{array}$ & $\begin{array}{l}\mathrm{Ti}^{*} \\
\%\end{array}$ & $\begin{array}{c}\mathrm{Tl} \\
\mathrm{ppm}\end{array}$ & $\begin{array}{c}U \\
\mathrm{ppm}\end{array}$ & $\begin{array}{c}\mathrm{V} \\
\mathrm{ppm}\end{array}$ & $\begin{array}{l}W^{*} \\
\mathrm{ppm}\end{array}$ & $\begin{array}{c}\mathrm{Y} \\
\mathrm{ppm}\end{array}$ & $\begin{array}{c}\mathrm{Zn} \\
\mathrm{ppm}\end{array}$ & $\begin{array}{l}\mathrm{Zr}^{*} \\
\mathrm{ppm}\end{array}$ & $\begin{array}{c}\text { Pulp Wt } \\
\text { grams }\end{array}$ & $\begin{array}{c}\text { Lab } \\
\text { Report }\end{array}$ \\
\hline 3 & 0.57 & 0.92 & 8.4 & 52.6 & 1200 & 10.7 & 55.6 & 0.002 & 0.08 & 1.11 & 2 & 1.40 & 160.0 & \begin{tabular}{l|l}
0.59 \\
\end{tabular} & $<0.05$ & 6.8 & 0.397 & 0.30 & 1.9 & 116 & 1.1 & 22.6 & 166 & 60.4 & 28 & FA04030730 \\
\hline 5 & 3.92 & 42 & 14.6 & 66.7 & 1640 & 18.5 & 39.2 & 0.003 & 0.01 & 4.49 & 3 & 3.30 & 06.5 & 0.95 & 0.09 & 4.1 & 0.845 & 0.28 & 4.7 & 308 & 1.0 & 22.0 & 162 & 108.5 & 22 & FA04030730 \\
\hline 7 & 3.25 & 0.51 & 18.8 & 66.3 & 1690 & 14.2 & 40.1 & 0.002 & 0.03 & 4.37 & 3 & 5.30 & 108.5 & 1.19 & 0.08 & 5.0 & 1.265 & 0.27 & 3.7 & 284 & 0.8 & 22.7 & 231 & 121.5 & $\overline{32}$ & FA04030730 \\
\hline 8 & 3.11 & 0.44 & 16.1 & 82.6 & 2520 & 12.6 & 34.5 & 0.002 & 0.01 & 6.27 & 3 & 2.00 & 116.0 & 1.01 & 0.08 & 4.4 & 1.120 & 0.29 & 4.6 & 314 & 0.8 & 21.9 & 240 & 112.5 & 24 & FA04030730 \\
\hline 9 & 0.78 & .02 & 10.1 & 36.5 & 1700 & 12.8 & 40.7 & 0.002 & 0.04 & 2.17 & 2 & 1.70 & 163.5 & 0.69 & $<0.05$ & 7.7 & 0.524 & 0.25 & 2.0 & 138 & 0.9 & 18.5 & 129 & 66.3 & 25 & \\
\hline 10 & 2.21 & 0.67 & 16.1 & 60.7 & 2140 & 10.9 & 35.8 & 0.003 & 0.02 & 4.36 & 3 & 1.70 & 129.5 & \begin{tabular}{|c|}
1.08 \\
\end{tabular} & 0.08 & 5.9 & 1.115 & 0.27 & 3.4 & 270 & 0.9 & 20.7 & 194 & 109.0 & 53 & FA04030730 \\
\hline 34 & 1.30 & 1.17 & 9.6 & 26.2 & 740 & 14.4 & 65.6 & $<0.002$ & 0.03 & 2.00 & 2 & 1.80 & 174.5 & 0.72 & 0.05 & 8.7 & 0.392 & 0.37 & 2.7 & 146 & 1.3 & 15.1 & 86 & 64.1 & & 0730 \\
\hline 47 & 0.65 & 1.48 & 9.6 & 31.2 & 710 & 14.8 & 60.3 & $<0.002$ & 0.03 & 1.22 & 2 & 1.70 & 213.0 & \begin{tabular}{|l|}
0.71 \\
\end{tabular} & $<0.05$ & 8.1 & 0.412 & 0.35 & 2.1 & 129 & 1.2 & 16.2 & 93 & 58.0 & $\sqrt{2}$ & $\overline{\text { FA0403 }}$ \\
\hline 48 & 0.86 & 1.50 & 10.4 & 35.1 & 780 & 12.8 & 56.9 & 0.002 & 0.03 & 1.22 & 2 & 5.70 & 228.0 & 0.77 & $<0.05$ & 10.0 & 0.431 & 0.32 & 2.4 & 126 & 1.3 & 18.1 & 80 & 65.7 & $\sqrt{32}$ & FA04030730 \\
\hline 49 & 0.73 & 1.52 & 10.4 & 32.8 & 790 & 11.9 & 56.5 & $<0.002$ & 0.03 & 1.14 & & 1.50 & 229.0 & 0.76 & $<0.05$ & \begin{tabular}{|l|}
9.7 \\
\end{tabular} & 0.436 & 0.32 & 2.3 & 124 & 1.2 & 17.4 & 79 & 64.8 & & FA040 \\
\hline 50 & 0.63 & 1.50 & 9.5 & 30.8 & 700 & 14.6 & 60.9 & 0.002 & 0.03 & 1.14 & 2 & 1.60 & 215.0 & 0.68 & $<0.05$ & 8.0 & 0.401 & 0.36 & 1.9 & 126 & 1.2 & 15.7 & 92 & 54.3 & 37 & FA04 \\
\hline 52 & 11.55 & 0.99 & 6.5 & 20.0 & 810 & 42.0 & 105.0 & 0.002 & 0.21 & 2.66 & 3 & 2.70 & 163.5 & 0.44 & 0.41 & 8.4 & 0.298 & 0.85 & 2.9 & 118 & 7.9 & 11.0 & 68 & 53.3 & 26 & FA04030730 \\
\hline 57 & 6.85 & 1.06 & 10.2 & 47.0 & 1130 & 73.9 & 84.0 & 0.004 & 0.16 & 3.87 & 3 & 18.80 & \begin{tabular}{l|l|}
173.5 \\
\end{tabular} & 0.67 & 0.15 & 9.5 & 0.427 & 0.62 & 2.5 & 149 & 3.6 & 18.0 & 311 & 63.7 & & \\
\hline 58 & 15.65 & 0.83 & 9.8 & 48.3 & 1310 & 93.2 & 82.4 & 0.006 & 0.24 & 5.60 & 2 & 8.30 & 130.5 & 0.66 & 0.24 & 9.5 & 0.415 & 0.66 & 2.5 & 177 & 3.6 & 18.2 & 529 & 62.0 & 1 & FA04 \\
\hline 61 & 2.64 & 1.22 & 9.5 & 37.6 & 920 & 28.7 & 66.1 & 0.004 & 0.06 & 1.68 & 2 & 1.90 & 192.0 & 0.65 & 0.06 & 8.3 & 0.377 & 0.46 & 2.3 & 127 & 1.2 & 17.9 & 142 & 61.8 & 55 & FA04030730 \\
\hline & 13 & 0.71 & 6.5 & 46.8 & 1060 & 81.8 & 68.8 & 0.002 & 0.08 & 4.70 & 3 & 00 & 119.5 & 0.45 & 0.06 & 5.5 & 0.339 & 0.53 & 1.8 & 47 & 2.3 & 19.3 & 245 & 55.6 & & FAO \\
\hline 66 & 3.60 & 0.85 & 8.5 & 41.6 & 910 & 43.2 & 124.5 & 0.006 & 0.12 & 7.86 & 2 & 4.40 & 88.9 & 0.55 & 0.57 & 6.3 & 0.395 & 0.98 & 2.4 & 260 & 4.2 & 14.3 & 164 & 83.9 & 7 & FA04 \\
\hline$\overline{6}$ & 1.24 & 1.25 & 10.2 & 33.5 & 800 & 16.4 & 67.1 & 0.003 & 0.03 & 1.44 & $\overline{2}$ & $\overline{1.9}$ & 184.5 & 0.73 & 0.05 & 10.1 & 0.415 & 0.39 & 2.6 & 141 & 1.3 & 17.6 & 84 & 66.7 & & 730 \\
\hline$\overline{6}$ & 1.07 & 1.32 & 10.7 & 33.3 & 790 & 14.5 & 68.3 & 0.003 & 0.03 & 1.24 & 2 & 2.10 & 193.0 & \begin{tabular}{|l|l|}
0.74 \\
\end{tabular} & $<0.05$ & 9.4 & 0.429 & 0.40 & 2.3 & & 1.3 & 16.8 & & 69.0 & & 730 \\
\hline 71 & 0.71 & 1.32 & 9.7 & 29.2 & 790 & 12.4 & 61.7 & 0.004 & 0.04 & 1.11 & 2 & 1.70 & 201.0 & 0.66 & $<0.05$ & 8.5 & 0.378 & 0.36 & 2.1 & 121 & 1.1 & 15.4 & 78 & 61.8 & 2 & FA040 \\
\hline 73 & 0.81 & 1.39 & 10.5 & 31.5 & 830 & 15.1 & 70.6 & 0.002 & 0.03 & 1.46 & 2 & 2.10 & 210.0 & 0.78 & $<0.05$ & 8.9 & 0.427 & 0.40 & 2.1 & 133 & 1.3 & 16.4 & 93 & 65.5 & & FA04 \\
\hline 74 & 0.96 & 1.34 & 10.6 & 32.4 & 800 & 14.4 & 72.4 & 0.002 & 0.03 & 1.40 & 2 & 2.0 & 202.0 & 0.73 & $<0.05$ & 9.5 & 0.410 & 0.43 & 2.3 & & 1.5 & 16.7 & 90 & 62.7 & & $\overline{\mathrm{FAO} 2}$ \\
\hline 76 & 1.60 & 1.12 & 10.8 & 23.4 & 610 & 15.5 & 76.3 & $<0.002$ & 0.02 & 0.94 & & 2.20 & 154.5 & 0.72 & 0.05 & 7.9 & 0.392 & 0.47 & 2.5 & 132 & 1.3 & 14.0 & 77 & 86.6 & 1 & FA04030730 \\
\hline 8 & 1.30 & 1.29 & 10.1 & 22.9 & 580 & 13.4 & 64.7 & 0.003 & 0.03 & 0.89 & & 2.6 & 178.0 & 0.72 & 0.05 & 7.7 & 0.408 & 0.37 & 2.3 & & 1.1 & 15.0 & & 77.3 & & FA04 \\
\hline & 1.06 & 1.36 & 11.1 & 25.5 & 640 & 13.3 & 60.7 & 0.003 & 0.02 & 1.00 & 2 & 1.80 & 197.0 & 0.79 & $<0.05$ & 9.0 & 0.417 & 0.36 & 2.4 & 123 & 1.4 & 16.8 & 74 & 77.1 & & FA04 \\
\hline 8 & 2.23 & 0.95 & 10.4 & 34.1 & 810 & 17.0 & 65.4 & 0.003 & 0.03 & 1.24 & & 3.30 & 144.0 & 0.77 & 0.07 & 8.1 & 0.408 & 0.39 & 2.9 & 147 & 1.3 & 16.1 & 87 & 67.0 & & FA04 \\
\hline 87 & 0 & 1.49 & 10.4 & 29.5 & 810 & 12.4 & 58.1 & 0.003 & 0.02 & 1.07 & 2 & 1.6 & 236.0 & 0.77 & $<0.05$ & 9.2 & 0.402 & 0.35 & 2.4 & & 1. & 18.9 & & 73.1 & & 730 \\
\hline & 0.87 & 1.53 & 12.0 & 29.4 & 870 & 12.8 & 57.3 & 0.003 & 0.02 & 1.14 & & 1.7 & 241.0 & 0.93 & $<0.05$ & 10.7 & 0.457 & 0.33 & 2.7 & 12 & 1.5 & 20.8 & 87 & 80.0 & & FA04 \\
\hline$\overline{9 x}$ & 1.02 & 1.45 & 12.2 & 33.4 & 850 & 14.0 & 65.8 & 0.002 & 0.02 & 1.21 & & 1.80 & 228.0 & 0.89 & $<0.05$ & 11.3 & 0.447 & 0.37 & 2.9 & 133 & 1.4 & 21.4 & 89 & 85.0 & & 730 \\
\hline & 10 & 1.50 & 11.2 & 31.5 & 860 & 13.4 & 61.0 & 0.003 & 0.02 & 1.13 & 2 & 1.7 & 235.0 & \begin{tabular}{|l|l|}
0.80 \\
\end{tabular} & $<0.05$ & 10.2 & 0.425 & 0.38 & 2.6 & & 1.4 & 19.4 & 91 & 75.4 & & \\
\hline 9 & 1.16 & 1.46 & 11.2 & 29.0 & 830 & 13.6 & 57.5 & 0.003 & 0.02 & 1.14 & & 1.60 & 230.0 & \begin{tabular}{|l|l|}
0.79 \\
\end{tabular} & 0.05 & 10.6 & 0.447 & 0.35 & 2.8 & 132 & 1.5 & 19.6 & 86 & 83.8 & 7 & 730 \\
\hline$\overline{93}$ & 2.76 & 0.64 & 10.0 & 34.9 & 1940 & 11.1 & 72.7 & 0.008 & 0.06 & 2.37 & 4 & 1.80 & 117.5 & 0.76 & 0.05 & 9.5 & 0.397 & 0.66 & 3.6 & 150 & 1.2 & 22.2 & 11 & 76.3 & & 730 \\
\hline & 2.66 & 0.61 & 10.2 & 49.4 & 1420 & 14.9 & 98.5 & & 0.06 & 2.31 & & & 128.0 & 0.74 & 0.08 & 11.4 & 422 & 0.71 & 3.4 & & & 19.0 & 125 & 78.0 & & \\
\hline$\overline{9}$ & 0.89 & 1.07 & 11.4 & 33.2 & 570 & 24.1 & 110.0 & 0.002 & 0.03 & 2.11 & & 2.60 & 183.5 & 0.83 & $<0.05$ & 14.0 & 0.398 & 0.54 & 2.8 & 114 & 1.6 & 17.0 & 91 & 81.4 & & FA04030730 \\
\hline 99 & 1.57 & 0.80 & 11.4 & 41.7 & 890 & 12.3 & 78.9 & $<0.002$ & 0.02 & 1.80 & $<1$ & 1.70 & 134.5 & \begin{tabular}{|l|l|}
0.84 \\
\end{tabular} & $<0.05$ & 11.2 & 0.611 & 0.50 & 2.3 & 114 & 1.3 & 14.8 & 90 & 94.5 & 6 & FA040 \\
\hline & 1.22 & 1.20 & 9.9 & & 570 & 15.8 & & $<0.002$ & 0.04 & 1.37 & & & 182.0 & \begin{tabular}{l|l}
0.73 \\
\end{tabular} & 0.05 & 7.5 & & & 2.2 & & & & & 68.1 & & \\
\hline 102 & 5.93 & 0.66 & 10.5 & 35.0 & 1220 & 27.1 & 92.3 & $<0.002$ & 0.05 & 2.98 & 2 & 7.20 & 134.5 & 0.78 & 0.16 & 9.6 & 0.418 & 0.66 & 4.8 & 43 & 1.6 & 22.2 & 83 & 95.1 & 12 & FA04C \\
\hline 104 & 3.67 & 1.05 & 11.6 & 34.2 & 1260 & 25.6 & 94.9 & $<0.002$ & 0.07 & 1.74 & $<1$ & 2.20 & 169.5 & 0.88 & 0.08 & 10.5 & 0.464 & 0.57 & 2.8 & 167 & 1.5 & 18.8 & 90 & 85.0 & 18 & 730 \\
\hline & 5.83 & 0.64 & 8.9 & 39.9 & 1590 & 31.4 & 77.9 & $<0.002$ & 0.10 & 2.56 & $\overline{-1}$ & & 115.5 & 0.69 & 0.14 & 8.4 & 0.423 & 0.55 & 4.4 & & 1.4 & 19.8 & 104 & 75.7 & & FA04 \\
\hline 110 & 4.09 & 0.90 & 10.7 & 39.3 & 890 & 25.9 & 83.3 & $<0.002$ & 0.05 & 6.38 & $<$ & 2.10 & 144.0 & 0.77 & 0.10 & 9.0 & 0.502 & 0.54 & 2.5 & 164 & 1.6 & 14.4 & 138 & 81.5 & 21 & 730 \\
\hline & 1.92 & 1.18 & 10.4 & 30.5 & 800 & 18.5 & 69.8 & $<0.002$ & 0.04 & 1.76 & $<1$ & & 176.5 & 0.15 & 0.07 & 8.5 & 0.454 & 0.47 & 2.4 & 143 & 1.1 & 14.4 & 86 & 16.7 & & \\
\hline 117 & 2.33 & 1.11 & 9.8 & 26.1 & 710 & 15.9 & 72.6 & $<0.002$ & 0.04 & 1.52 & 1 & 1.70 & 172.5 & 0.73 & 0.06 & 7.2 & 0.402 & 0.46 & 2.3 & & 1.1 & 13.1 & $\overline{92}$ & 69.4 & 11 & FA04030730 \\
\hline 119 & 0.84 & 1.53 & 9.5 & 39.6 & 730 & 11.0 & 59.3 & $<0.002$ & 0.03 & 0.88 & $<1$ & 1.80 & 235.0 & \begin{tabular}{|l|l|}
0.72 & \\
\end{tabular} & $<0.05$ & 7.2 & 0.447 & 0.36 & 2.0 & 122 & 0.9 & 14.4 & 110 & 66.6 & 44 & FA04030730 \\
\hline
\end{tabular}


Table 1. Location and New Trace Element Geochemical Results for 902 Stream-Sediment Samples collected in the Livengood Area, Livengood Quadrangle, Alaska IS = Insufficient Sample for Analysis

\begin{tabular}{|c|c|c|c|c|c|c|c|c|c|c|c|c|c|c|c|c|c|c|c|c|c|c|c|c|c|c|c|}
\hline MPLE & ITM E & TM N & $\begin{array}{l}\mathrm{Au} \\
\mathrm{ppm}\end{array}$ & $\begin{array}{c}\mathrm{Ag} \\
\mathrm{ppm}\end{array}$ & $\begin{array}{l}\text { Al } \\
\%\end{array}$ & $\begin{array}{c}\text { As } \\
\text { ppm }\end{array}$ & $\begin{array}{l}\mathrm{Ba}^{*} \\
\mathrm{ppm}\end{array}$ & $\begin{array}{c}\mathrm{Be} \\
\mathrm{ppm}\end{array}$ & $\begin{array}{c}\mathrm{Bi} \\
\mathrm{ppm}\end{array}$ & $\begin{array}{c}\mathrm{Ca} \\
\% \\
\end{array}$ & \begin{tabular}{|c|}
$\mathrm{Cd}$ \\
$\mathrm{ppm}$ \\
\end{tabular} & $\begin{array}{r}\text { Ce } \\
\text { ppm }\end{array}$ & $\begin{array}{l}\text { Co } \\
\text { ppm }\end{array}$ & $\begin{array}{l}\mathrm{Cr}^{*} \\
\mathrm{ppm}\end{array}$ & $\begin{array}{r}\text { Cs } \\
\text { ppm }\end{array}$ & $\begin{array}{r}\mathrm{Cu} \\
\mathrm{ppm}\end{array}$ & $\begin{array}{c}\mathrm{Fe} \\
\% \\
\end{array}$ & $\begin{array}{l}\mathrm{Ga} \\
\mathrm{ppm}\end{array}$ & $\begin{array}{l}\mathrm{Ge} \\
\mathrm{ppm}\end{array}$ & $\begin{array}{c}\mathrm{Hf} \\
\mathrm{ppm} \\
\end{array}$ & $\begin{array}{l}\mathrm{Hg} \\
\mathrm{ppm}\end{array}$ & $\begin{array}{c}\text { In } \\
\text { ppm }\end{array}$ & $\begin{array}{l}\mathrm{K} \\
\% \\
\end{array}$ & $\begin{array}{c}\mathrm{La} \\
\mathrm{ppm}\end{array}$ & $\begin{array}{c}\mathrm{Li} \\
\mathrm{ppm} \\
\end{array}$ & $\begin{array}{c}\mathrm{Mg} \\
\% \\
\end{array}$ & $\begin{array}{l}\mathrm{Mn} \\
\mathrm{ppm}\end{array}$ \\
\hline 120 & 2700 & 77941 & $<0.005$ & 0.13 & \begin{tabular}{|l|l|}
6.05 \\
\end{tabular} & 14.9 & 710 & 1.24 & 0.23 & 0.88 & 0.36 & & 24.1 & 109 & 3.39 & 34.7 & 4.09 & 14.80 & 0.19 & \begin{tabular}{|l|}
2.2 \\
\end{tabular} & 0.38 & 0.051 & \begin{tabular}{|l|}
1.51 \\
\end{tabular} & 28.4 & 19.8 & 1.12 & 1790 \\
\hline 23 & & & 09 & & \begin{tabular}{|l}
6.03 \\
\end{tabular} & 9.1 & 360 & .00 & 0.1 & .52 & \begin{tabular}{|l|}
0.31 \\
\end{tabular} & 66.5 & 14.8 & & 2.6 & 3.6 & 3.21 & 13.80 & 0.19 & 2.0 & 0.23 & 0.043 & \begin{tabular}{|l|}
1.32 \\
\end{tabular} & 31.9 & 7.8 & \begin{tabular}{|l|l} 
\\
\end{tabular} & 973 \\
\hline 5 & & & 0.005 & 0.07 & 8.09 & 9.6 & 970 & 1.90 & 0.27 & 1.00 & 0.26 & 103.5 & 14.5 & 94 & 5.51 & 28.2 & 3.34 & 21.10 & 0.24 & 3.2 & 0.93 & 0.056 & \begin{tabular}{|l}
2.75 \\
\end{tabular} & 51.4 & 8.0 & 0.79 & 323 \\
\hline & & & & & & 8.3 & 310 & & & & & & & & & & & & & & & & 1.46 & & & 0.92 & \\
\hline 74 & & & 0.007 & .05 & 5.71 & 2.6 & 700 & 0.96 & 0.09 & 1.36 & \begin{tabular}{|l|}
0.11 \\
\end{tabular} & 51.4 & 7.1 & 76 & 2.23 & 8.6 & 2.08 & 14.00 & 0.16 & 1.3 & 0.19 & 0.036 & \begin{tabular}{|l|}
1.32 \\
\end{tabular} & 25.4 & 19.4 & 0.86 & 317 \\
\hline & & & .005 & 0.09 & 6.28 & 16.7 & 730 & .08 & 0.15 & $\begin{array}{ll}1.32 \\
\end{array}$ & 0.18 & 51.8 & 10.4 & 79 & 2.50 & 14.1 & 3.54 & 14.80 & 0.19 & 1.5 & 0.09 & 0.044 & 1.34 & 25.7 & 21.8 & 0.98 & 351 \\
\hline & & & 2005 & & \begin{tabular}{|l|l|}
5.41 \\
\end{tabular} & 22.4 & 670 & & 0.14 & 1.50 & 0.19 & 65.6 & 14.3 & & 2.06 & 14.9 & 5.39 & 12.70 & 0.24 & & 018 & & 1.08 & 31.9 & & 0.87 & \\
\hline & & & 0.009 & 0.10 & \begin{tabular}{|l|l|}
6.18 \\
\end{tabular} & 8.3 & 720 & 1.04 & 0.14 & 1.48 & \begin{tabular}{|l|l}
0.17 \\
\end{tabular} & 64.9 & 12.0 & 89 & 2.28 & 17.4 & 3.14 & 13.75 & 0.21 & 1.6 & 0.08 & 0.040 & \begin{tabular}{|l}
1.22 \\
\end{tabular} & 31.5 & \begin{tabular}{|l|}
27.8 \\
\end{tabular} & 1.02 & 539 \\
\hline & & & 0.005 & 10 & 5.94 & 7.4 & 700 & 0.96 & 0.12 & 1.48 & 0.18 & 63.8 & 11.3 & & 2.15 & 16.1 & 3.01 & 13.35 & 0.20 & 1.6 & 0.10 & 0.043 & \begin{tabular}{|l|l|}
1.15 \\
\end{tabular} & 31.0 & 22.9 & 1.00 & 500 \\
\hline & & & 0.007 & 09 & \begin{tabular}{|l|l}
5.97 \\
\end{tabular} & 9.1 & 720 & 1.07 & 0.14 & 1.60 & \begin{tabular}{|l|l|}
0.26 \\
\end{tabular} & 68.8 & 15.5 & 9 & 2.28 & 19.3 & 3.41 & 13.65 & 0.20 & 1.7 & 0.12 & 0.048 & \begin{tabular}{|l|l|}
1.14 \\
\end{tabular} & 33.1 & \begin{tabular}{|l|l|}
48.5 \\
\end{tabular} & \begin{tabular}{|l}
1.07 \\
\end{tabular} & 735 \\
\hline & & & 0.008 & 0.11 & \begin{tabular}{|l|l|}
6.10 \\
\end{tabular} & 11.0 & 790 & 1.12 & 0.16 & 1.42 & \begin{tabular}{|l|l}
0.23 \\
\end{tabular} & 71.4 & 14.3 & 9 & 2.68 & 21.1 & 3.27 & 15.10 & \begin{tabular}{|l|l|}
0.22 \\
\end{tabular} & 1.8 & 0.21 & 0.050 & \begin{tabular}{|l|l}
1.27 \\
\end{tabular} & 34.4 & \begin{tabular}{|c|}
30.1 \\
\end{tabular} & 0.98 & 659 \\
\hline & & & 0.008 & 12 & 5.88 & 11.2 & 740 & .16 & 0.18 & 1.38 & \begin{tabular}{|l|l}
0.22 \\
\end{tabular} & 7 & 12.6 & & 6.3 & $\angle 1.8$ & 3.26 & 13.50 & \begin{tabular}{|l}
0.22 \\
\end{tabular} & 1.6 & 0.15 & 0.046 & \begin{tabular}{|l|}
1.14 \\
\end{tabular} & 34.5 & 40.2 & 0.95 & 559 \\
\hline & & & $<0.005$ & 14 & 59 & 8.3 & 20 & $\overline{12}$ & 0.15 & 0.90 & 0.26 & 5. & 13. & 2 & 4.8 & 6. & 3.65 & 15.75 & 0.20 & 1.8 & 0.32 & 0.052 & 1.42 & 31.7 & 50.7 & 1.10 & 64 \\
\hline & & & 0.005 & 0.08 & 5.78 & 5.7 & 730 & 1.05 & 0.12 & 1.60 & \begin{tabular}{|l|l|}
0.18 \\
\end{tabular} & 69.7 & 10.5 & 83 & 2.12 & 15.7 & 2.66 & 13.60 & 0.23 & 1.8 & 0.15 & 0.040 & 1.19 & 33.3 & \begin{tabular}{|l|}
30.5 \\
\end{tabular} & 0.98 & 450 \\
\hline & & & & .08 & 6.00 & 50 & 770 & & & \begin{tabular}{|l|}
1.64 \\
\end{tabular} & \begin{tabular}{|l|l|}
0.20 \\
\end{tabular} & & 12.4 & & 2.39 & 6.3 & 3.09 & 4.65 & 0.22 & $\bar{E}$ & 0.16 & 0.045 & \begin{tabular}{|l|}
1.30 \\
\end{tabular} & 42.1 & 27.9 & \begin{tabular}{|l|l}
1.07 \\
\end{tabular} & 563 \\
\hline & & & 0.005 & 0.09 & 5.87 & 8.5 & 700 & 0.99 & 0.13 & 1.44 & 0.20 & 65.7 & 11.4 & 84 & 2.16 & 17.5 & 2.95 & 13.30 & 0.22 & 1.5 & 0.31 & .040 & \begin{tabular}{|l|l} 
\\
\end{tabular} & 31.9 & 22.3 & 0.95 & 180 \\
\hline & & & 0.006 & 0.09 & 5.32 & 7.7 & 650 & .06 & 0.13 & 1.40 & 0.20 & 5.7 & 10.8 & & 2.02 & 16. & 2.77 & 12.85 & 0.20 & 1.5 & 1.01 & .040 & 1.03 & 31.6 & 26.4 & 0.90 & 463 \\
\hline & & & 0.007 & 93 & 5.84 & 7.2 & 90 & & & 1.20 & $\overline{10}$ & & 8. & & & & 2.90 & 13.45 & 0.19 & & 0.15 & .093 & 1.38 & 25.0 & 19.7 & 0.9 & $\overline{13}$ \\
\hline & & & 0.008 & 0.89 & 6.26 & 21.6 & 780 & 1.13 & 0.39 & 1.44 & 1.92 & 61.2 & 15.5 & 9 & 4.44 & 30.9 & 3.12 & 14.20 & 0.21 & 1.7 & 0.21 & 076 & 1.33 & 29.5 & 23.5 & 1.03 & 1205 \\
\hline & & & 111 & 69 & 91 & 9.8 & 740 & & 0.39 & \begin{tabular}{|l|}
1.33 \\
\end{tabular} & 2.24 & & 23.7 & & 3.1 & 32. & 3.17 & 13.40 & 0.20 & 1.6 & 0.16 & 0.073 & 1.26 & 28.7 & \begin{tabular}{|l|}
19.2 \\
\end{tabular} & 0.96 & 2170 \\
\hline & & & 0.039 & 54 & 5.80 & 23.4 & 720 & 0.99 & 0.34 & 1.36 & \begin{tabular}{|l}
1.56 \\
\end{tabular} & & 13. & & 3.54 & 290 & 3.01 & 3.60 & 0.22 & 17 & 0.17 & 0.068 & 1.24 & 28.9 & 22.1 & 0.98 & 880 \\
\hline & & & 0.010 & 0.57 & 5.93 & 22.4 & 750 & 1.08 & 0.37 & 1.38 & \begin{tabular}{|l|}
1.50 \\
\end{tabular} & 61.8 & 14.2 & 9 & 3.94 & 29.8 & 2.94 & 14.25 & 0.19 & 1.6 & 0.13 & 0.068 & \begin{tabular}{|l|l}
1.32 \\
\end{tabular} & 30.4 & 26.0 & 1.00 & 941 \\
\hline & & & $\overline{006}$ & 15 & 5.90 & 6.5 & 940 & & & 1.77 & 0.43 & 6. & 14. & & 2.8 & 31. & 3.37 & 3.80 & 0.24 & 2.0 & 0.19 & .048 & \begin{tabular}{|l|}
1.11 \\
\end{tabular} & 28.0 & \begin{tabular}{|l|l}
17.2 \\
\end{tabular} & 1.11 & 666 \\
\hline & & & 0 & & 6.22 & 10.1 & 840 & & & 58 & & 57 & 12. & & 3.7 & & 3.59 & 4.70 & & 2.0 & & & 1.20 & 28 & \begin{tabular}{|l|l|}
17.8 \\
\end{tabular} & 1.0 & 13 \\
\hline & & & 0.005 & .19 & 6.67 & 12.5 & 920 & & 0.18 & 2.00 & 0.66 & & 19. & & 3.24 & 59. & 4.85 & 16.80 & 0.26 & 2. & 0.19 & .068 & 1.22 & 25.7 & 19.3 & 1.43 & 947 \\
\hline & & & 0 & 21 & & 11.2 & & & & 2.02 & & & & & $3 . \angle 6$ & 4.5 & 4.50 & 6.25 & 0.24 & 2. & 12 & 061 & 1.18 & 6.8 & 19.9 & 1.38 & $\overline{924}$ \\
\hline & & & $\overline{006}$ & 18 & 6.52 & 8.7 & 900 & 1.27 & 0.14 & 1.78 & 0.44 & 58.8 & 13. & & 2.69 & 37.3 & 3.75 & 15.60 & 0.24 & 2.5 & 0.16 & 050 & \begin{tabular}{|l|}
1.24 \\
\end{tabular} & 28.5 & 8.6 & 1.13 & 620 \\
\hline & & & & & 30 & 7.6 & 880 & & & 1.86 & 46 & 56 & 14. & & 2.7 & 35.5 & 3.45 & 15.60 & 0.26 & 2.3 & 0.30 & .048 & \begin{tabular}{|l|}
1.18 \\
\end{tabular} & 27.2 & \begin{tabular}{|l|l|}
18.0 \\
\end{tabular} & 1.09 & 776 \\
\hline & & & & 12 & 5.97 & 7 & 910 & & & & & & & & & & 3.0 & 1.10 & & 2. & & & & & 28.1 & 0.95 & 63 \\
\hline & & & & & & & & & & & & & & & & & 3.2 & 525 & & & & & & 35 & & 106 & 341 \\
\hline & & & .005 & 0.08 & \begin{tabular}{|l|l|}
6.03 \\
\end{tabular} & 11.8 & 830 & 1.48 & 0.18 & 1.57 & .31 & 77.2 & 14.2 & & 3.44 & 22.4 & 3.20 & 15.80 & 0.22 & 2.1 & 0.10 & .048 & 1.32 & 38.2 & 36.0 & 0.90 & 813 \\
\hline & & & & 0.08 & 5.99 & 9.1 & 870 & 1. & 0. & \begin{tabular}{|l|}
1.62 \\
\end{tabular} & \begin{tabular}{|l|l}
0.35 \\
\end{tabular} & 68 & & & 2.78 & 17.7 & 2.94 & 14.25 & 24 & 2.1 & 0.17 & .041 & 1.29 & 33.4 & 26.5 & 0.93 & 80 \\
\hline & & & 007 & & 5.96 & & 920 & & & & & & & & 2.63 & & 3.25 & 4.15 & & & 0.07 & & & 32.0 & 20.7 & 0.96 & $\overline{654}$ \\
\hline & & 7 & 0.006 & 0.12 & 5.71 & 6.3 & 890 & 1.30 & 0.14 & \begin{tabular}{|l|}
1.62 \\
\end{tabular} & \begin{tabular}{|l}
0.26 \\
\end{tabular} & 58.3 & 10.2 & & 2.50 & 18.7 & 2.67 & 14.00 & 0.21 & 1.8 & 0.23 & 0.038 & 1.28 & 28.4 & 27.5 & \begin{tabular}{|l}
0.92 \\
\end{tabular} & 428 \\
\hline & & & & & & 4 & 900 & & & \begin{tabular}{|c|}
1.66 \\
\end{tabular} & & & & & & & 2.68 & & & 1.9 & 0.14 & & 1.28 & 28.8 & .5 & 0.95 & 22 \\
\hline & & & 0 & & & 8 & 850 & & & 1.80 & & & & & 2. & & 2.78 & 3.80 & & 1.9 & 0.09 & .039 & 1.28 & 28.8 & 21.2 & 0.93 & 507 \\
\hline & & $2 / 1$ & 0.006 & 0.10 & 5.46 & 9.5 & 860 & 1.2 & 0.1 & \begin{tabular}{|l|}
1.92 \\
\end{tabular} & 0.34 & 63.8 & 12.5 & & 2.28 & 1.3 & 2.90 & 13.50 & 0.25 & 2.2 & 0.07 & .038 & \begin{tabular}{|l|}
1.23 \\
\end{tabular} & 30.9 & 24.7 & 0.98 & 812 \\
\hline & & & & & & & & & & & & & & & & & & & & & 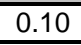 & & & 317 & 0 & L.04 & 27 \\
\hline & & & .005 & 0.12 & 6.19 & 9.0 & 930 & & 0. & 2.03 & & & 1. & & 2.41 & & 3.24 & 13.95 & 0.23 & 2.2 & 0.05 & 0.042 & \begin{tabular}{|l|l|}
1.34 \\
\end{tabular} & 29.9 & \begin{tabular}{|l|}
19.2 \\
\end{tabular} & 1.08 & 572 \\
\hline & & & .005 & \begin{tabular}{|l|}
0.14 \\
\end{tabular} & 5.50 & 8.3 & 830 & 1. & 0. & 1.88 & 0.35 & 63 & 11.6 & 8 & 2.3 & 20.0 & 2.88 & 13.45 & 0.24 & 2.0 & 0.10 & .040 & \begin{tabular}{|l|}
1.24 \\
\end{tabular} & 30.6 & 23.3 & \begin{tabular}{|l}
0.98 \\
\end{tabular} & 527 \\
\hline & & & & & & 8. & 92 & & & 2.06 & & & & & & & 3. & & & 2.. & 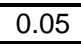 & & & 31.1 & -0 & \begin{tabular}{|l|l|} 
\\
\end{tabular} & 567 \\
\hline 250 & & 7270555 & 0.006 & 0.12 & 5.77 & 8.0 & 850 & 1.34 & 0.12 & 1.94 & 0.32 & 65.5 & 11.6 & 82 & 2.34 & 18.7 & 2.92 & 13.85 & 0.23 & 2.1 & 0.09 & 0.042 & $\begin{array}{ll}1.27 \\
\end{array}$ & 32.2 & 23.6 & 1.01 & 507 \\
\hline & 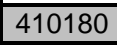 & 1261010 & 0.010 & \begin{tabular}{|l|}
0.12 \\
\end{tabular} & & 8.1 & 890 & & 0.17 & ... & & 02.1 & 11.6 & & 2.40 & $\angle 0.2$ & 3.04 & & & 2.1 & 0.06 & 0.040 & 1.30 & 31.1 & 24.0 & 1.03 & 551 \\
\hline
\end{tabular}


Table 1. Location and New Trace Element Geochemical Results for 902 Stream-Sediment Samples collected in the Livengood Area, Livengood Quadrangle, Alaska IS = Insufficient Sample for Analysis

\begin{tabular}{|c|c|c|c|c|c|c|c|c|c|c|c|c|c|c|c|c|c|c|c|c|c|c|c|c|c|c|}
\hline SAMPLE & $\begin{array}{l}\text { Mo } \\
\mathrm{ppm}\end{array}$ & $\begin{array}{c}\mathrm{Na} \\
\% \\
\end{array}$ & $\begin{array}{l}\mathrm{Nb} \\
\mathrm{ppm}\end{array}$ & $\begin{array}{c}\mathrm{Ni} \\
\mathrm{ppm}\end{array}$ & $\begin{array}{c}\mathrm{P} \\
\mathrm{ppm}\end{array}$ & $\begin{array}{l}\mathrm{Pb} \\
\mathrm{ppm}\end{array}$ & $\begin{array}{l}\mathrm{Rb} \\
\mathrm{ppm}\end{array}$ & $\begin{array}{l}\mathrm{Re} \\
\mathrm{ppm}\end{array}$ & $\begin{array}{l}\mathrm{S} \\
\%\end{array}$ & $\begin{array}{l}\mathrm{Sb} \\
\mathrm{ppm}\end{array}$ & $\begin{array}{l}\text { Se } \\
\mathrm{ppm}\end{array}$ & $\begin{array}{l}S^{\text {Sn }} \\
\mathrm{ppm}\end{array}$ & $\begin{array}{c}\mathrm{Sr} \\
\mathrm{ppm}\end{array}$ & $\begin{array}{l}\mathrm{Ta}^{*} \\
\mathrm{ppm}\end{array}$ & $\begin{array}{c}\text { Te } \\
\text { ppm }\end{array}$ & $\begin{array}{c}\text { Th } \\
\mathrm{ppm}\end{array}$ & $\begin{array}{l}\mathrm{Ti}^{*} \\
\%\end{array}$ & $\begin{array}{c}\mathrm{Tl} \\
\mathrm{ppm}\end{array}$ & $\begin{array}{c}U \\
\mathrm{ppm}\end{array}$ & \begin{tabular}{|c|}
$\mathrm{V}$ \\
$\mathrm{ppm}$
\end{tabular} & $\begin{array}{l}W^{*} \\
\mathrm{ppm}\end{array}$ & $\begin{array}{c}\mathrm{Y} \\
\mathrm{ppm}\end{array}$ & $\begin{array}{c}\mathrm{Zn} \\
\mathrm{ppm}\end{array}$ & $\begin{array}{l}\mathrm{Zr}^{*} \\
\mathrm{ppm}\end{array}$ & $\begin{array}{c}\text { Pulp Wt } \\
\text { grams }\end{array}$ & $\begin{array}{c}\text { Lab } \\
\text { Report }\end{array}$ \\
\hline 120 & 3.03 & 0.83 & 9.2 & 51.6 & 850 & 18.5 & 73.4 & $<0.002$ & 0.03 & 1.90 & $<1$ & 5.60 & 127.0 & 0.68 & 0.09 & 8.5 & 0.425 & 0.46 & 2.6 & 155 & 1.4 & 15.2 & 108 & 85.5 & 17 & FA04030730 \\
\hline 123 & 1.28 & & 9.9 & 36.0 & 780 & 13.2 & 60.2 & $<0.002$ & 0.03 & 1.18 & $<1$ & 1.60 & 0 & 0.73 & 0.05 & 8.8 & 0.451 & 0.41 & 2.4 & 132 & 1.5 & 16.5 & 91 & 75.1 & 53 & \\
\hline 165 & 0.68 & 0.83 & 13.2 & 35.8 & 610 & 16.1 & 143.0 & $<0.002$ & 0.04 & 0.73 & $<1$ & 2.60 & 144.5 & 1.08 & 0.05 & 18.6 & 0.476 & 0.69 & 2.9 & 104 & 1.6 & 19.4 & 87 & 126.0 & 20 & FA04030730 \\
\hline 167 & 1.66 & 0.75 & 9.8 & 41.5 & 790 & 13.1 & 79.3 & $<0.002$ & 0.03 & 1.73 & 1 & 1.70 & 134.5 & 0.73 & $<0.05$ & 10.4 & 0.424 & 0.52 & 2.3 & 101 & 1.2 & 14.4 & 95 & 80.3 & 19 & FA04030730 \\
\hline 174 & 0.51 & 42 & 8.9 & 20.4 & 390 & 9.8 & 62.2 & $<0.002$ & 0.04 & 0.82 & $<1$ & 1.80 & 198.5 & 0.67 & $<0.05$ & $\begin{array}{ll}6.9 \\
\end{array}$ & 0.402 & 0.38 & 1.5 & 89 & 1.1 & 10.4 & 52 & 51.1 & 17 & \\
\hline 175 & 0.88 & 1.30 & 8.5 & 26.8 & 720 & 11.4 & 72.4 & $<0.002$ & $\overline{0.04}$ & 0.92 & $<1$ & 1.70 & 189.5 & \begin{tabular}{|l|}
0.64 \\
\end{tabular} & $<0.05$ & 6.9 & 0.390 & 0.42 & 1.5 & 119 & 0.9 & 11.9 & 95 & 58.4 & 19 & FA04030730 \\
\hline 176 & 0.95 & 1.21 & 8.4 & 27.4 & 790 & 11.4 & 55.0 & $<0.002$ & 0.03 & 3.00 & $<1$ & 1.80 & 189.5 & 0.63 & $<0.05$ & 8.8 & 0.394 & 0.36 & 1.9 & 104 & 1.0 & 14.3 & 61 & 60.1 & 47 & 0730 \\
\hline 178 & 0.73 & 1.37 & 9.8 & 26.1 & 580 & 11.4 & 58.8 & $<0.002$ & 0.02 & 0.98 & $<1$ & 1.70 & 196.5 & 0.74 & $<0.05$ & 8.9 & 0.484 & 0.35 & 1.7 & 117 & 1.3 & 13.5 & 72 & 58.3 & 27 & FA040 \\
\hline 179 & 0.72 & 1.32 & 9.8 & 26.0 & 540 & 11.7 & 55.2 & $<0.002$ & 0.02 & 0.97 & $<1$ & 1.60 & 189.5 & 0.78 & $<0.05$ & 8.2 & 0.488 & 0.33 & 1.8 & 111 & 1.0 & 13.2 & 68 & 59.3 & 74 & FA04030730 \\
\hline 30 & 83 & 28 & 10.1 & 28.4 & 590 & 13.3 & 55.4 & $<0.002$ & 0.02 & 1.01 & $<1$ & 1.80 & 187.0 & 0.78 & $<0.05$ & 9.3 & 0.546 & 0.37 & 1.9 & 120 & 1.0 & 14.2 & & 65.2 & & 0730 \\
\hline 31 & 0.97 & 1.28 & 10.1 & 30.7 & 610 & 14.0 & 67.1 & $<0.002$ & 0.03 & 1.21 & 1 & 1.90 & 190.0 & 0.79 & $<0.05$ & 9.7 & 0.488 & 0.40 & 2.2 & 122 & 1.1 & 15.0 & 96 & 67.9 & 1 & FA04 \\
\hline 182 & 0.99 & 1.17 & 9.6 & 28.0 & 630 & 12.9 & 58.4 & $<0.002$ & 0.03 & 1.10 & $<1$ & 1.80 & 174.0 & 0.76 & $<0.05$ & 10.2 & 0.493 & 0.37 & 1.9 & 124 & 1.1 & 14.6 & 89 & 61.3 & 22 & FA0403 \\
\hline & & 1.29 & 10.0 & 45.8 & 910 & 17.6 & 70.9 & $<0.002$ & 0.02 & 0.86 & $<1$ & 1.60 & 139.5 & 0.76 & 0.05 & 8.0 & 0.523 & 0.44 & 1.8 & 156 & & 16.0 & 110 & 68.3 & & \\
\hline 186 & 0.56 & 1.46 & 9.8 & 28.1 & 610 & 11.0 & 55.9 & $<0.002$ & 0.02 & 0.94 & $<1$ & 1.60 & 213.0 & 0.74 & $<0.05$ & 8.6 & 0.433 & 0.35 & 1.9 & 105 & 1.1 & 15.5 & 79 & 64.8 & 3 & FA04 \\
\hline 187 & 0.69 & 1.48 & 11.4 & 30.7 & 690 & 12.5 & 61.5 & $<0.002$ & 0.02 & 1.07 & 1 & 1.70 & 214.0 & 0.92 & $<0.05$ & 10.9 & 0.517 & 0.38 & 2.2 & 122 & 1.5 & 17.0 & 77 & 77.1 & 2 & FA04030730 \\
\hline & 0.76 & 1.29 & 9.0 & 26.2 & 560 & 12.1 & 55.6 & $<0.002$ & 0.02 & 1.00 & & 1.50 & 186.5 & 0.68 & $<0.05$ & 8.9 & 443 & 0.37 & 1.9 & 111 & & 13.9 & & 56.4 & & \\
\hline 190 & 0.72 & 1.20 & 9.1 & 25.3 & 520 & 11.2 & 51.7 & $<0.002$ & 0.02 & 1.02 & $<1$ & 1.50 & 175.0 & 0.72 & $<0.05$ & 8.5 & 0.413 & 0.33 & 1.9 & 99 & 0.9 & 13.7 & 62 & 56.6 & $\overline{42}$ & FA04 \\
\hline$\overline{1}$ & 1.02 & 1.22 & 9.0 & 26.2 & 710 & 30.3 & 64.7 & $<0.002$ & 0.05 & 1.74 & $<1$ & 2.20 & 180.0 & \begin{tabular}{|l|}
0.66 \\
\end{tabular} & 0.06 & 6.6 & 0.425 & 0.50 & 1.7 & 117 & 1.6 & 11.2 & 112 & 59.4 & & \\
\hline & 0.87 & 1.31 & 9.7 & 32.8 & 750 & 36.3 & 64.1 & $<0.002$ & 0.03 & 2.06 & $<1$ & 2.20 & 204.0 & \begin{tabular}{|l|l}
0.71 \\
\end{tabular} & $<0.05$ & 7.9 & 436 & 0.51 & 1.8 & 123 & 1.4 & 14.9 & & 62.2 & & \\
\hline 96 & 1.00 & 1.23 & 9.0 & 30.6 & 750 & 27.6 & 63.7 & $<0.002$ & 0.04 & 1.42 & $<1$ & 2.00 & 189.5 & \begin{tabular}{l|l|}
0.66 \\
\end{tabular} & $<0.05$ & 7.5 & 0.421 & 0.49 & 1.8 & 118 & 1.6 & \begin{tabular}{|l|}
13.6 \\
\end{tabular} & 124 & 63.0 & 2 & FA040 \\
\hline & 84 & 1.24 & 9.1 & 30.3 & 670 & 22.3 & 61.6 & $<0.002$ & 0.03 & 1.36 & $<1$ & 2.00 & \begin{tabular}{|l|}
189.0 \\
\end{tabular} & \begin{tabular}{|l|}
0.70 \\
\end{tabular} & $<0.05$ & 7.6 & 0.428 & 0.47 & 1.8 & 117 & 2.1 & 13.4 & 119 & 61.7 & & FA04 \\
\hline & 84 & 1.29 & 9.3 & 30.6 & 680 & 26.7 & 66.1 & $<0.002$ & 0.03 & 1.52 & $<1$ & 2.00 & 198.5 & \begin{tabular}{|l|l|}
0.67 \\
\end{tabular} & 0.05 & 7.8 & 0.430 & 0.52 & 1.8 & 121 & 1.5 & 13.8 & 122 & 64. & & $\mathrm{FAO}$ \\
\hline & 1.46 & 1.30 & 11.0 & 32.5 & 780 & 12.3 & 54.7 & $<0.002$ & 0.04 & 0.93 & $<1$ & 1.60 & 200.0 & \begin{tabular}{|l|l}
0.79 \\
\end{tabular} & $<0.05$ & 6.7 & 0.558 & 0.39 & 2.1 & 148 & 1.1 & 16.9 & 106 & 81.4 & & FA04C \\
\hline & 93 & 1.03 & 9.6 & 36.2 & 830 & 12.9 & 65.0 & 0.002 & 0.08 & 1.25 & 1 & 1.80 & 178.0 & \begin{tabular}{|l|}
0.70 \\
\end{tabular} & 0.05 & 7.4 & 481 & 0.60 & 2.7 & & $\overline{0.9}$ & 19.0 & 15 & $\overline{80.2}$ & & $\overline{F A 0}$ \\
\hline & 4.03 & 1.21 & 11.6 & 41.6 & 740 & 12.5 & 61.1 & 0.002 & 0.08 & 1.28 & 1 & 2.50 & 189.0 & \begin{tabular}{|l|l|}
0.77 \\
\end{tabular} & 0.06 & 6.3 & 0.740 & 0.55 & 2.7 & 223 & 0.9 & 19.2 & 126 & 98.4 & & FA04 \\
\hline & 3.69 & 1.25 & 11.0 & 40.0 & 750 & 12.0 & 60.8 & 0.002 & 0.07 & 1.33 & 1 & 1.80 & 196.0 & \begin{tabular}{|c|}
0.78 \\
\end{tabular} & 0.06 & 6.8 & 0.678 & 0.51 & 2.6 & 209 & 0.9 & 20.0 & 137 & 96.7 & & FA04 \\
\hline & 15 & 1.46 & 11.4 & 30.6 & 700 & 11.5 & 57.4 & $<0.002$ & 0.05 & 1.27 & 1 & 1.70 & 223.0 & \begin{tabular}{|l|l}
0.75 \\
\end{tabular} & $<0.05$ & $\overline{7.4}$ & 0.603 & 0.48 & 2.6 & & 1. & 17.2 & 120 & 94 & & 730 \\
\hline & 1.99 & 33 & 11.6 & 32.2 & 760 & 11.8 & 60.0 & $<0.002$ & 0.04 & 1.06 & & 1.70 & 216.0 & \begin{tabular}{|l|}
0.76 \\
\end{tabular} & $<0.05$ & 7.5 & 0.558 & 0.46 & 2.3 & 15 & 1. & 16.9 & 102 & 89.5 & & FA04 \\
\hline & 1.02 & 1.40 & 11.2 & 27.7 & 800 & 13.2 & 64.3 & $<0.002$ & 0.04 & 1.02 & & 1.60 & 240.0 & \begin{tabular}{|l|l|}
0.77 \\
\end{tabular} & $<0.05$ & 8.6 & 0.431 & 0.41 & 2.3 & 21 & 1.3 & 15.5 & 96 & 84.2 & & 7730 \\
\hline & & 1.48 & 11.8 & 29.7 & 810 & 12.5 & 62.6 & $<0.002$ & 0.03 & 1.12 & & 1.80 & \begin{tabular}{|l|}
257.0 \\
\end{tabular} & \begin{tabular}{|c|}
0.83 \\
\end{tabular} & $<0.05$ & 9.5 & 0.496 & 0.39 & 2.3 & & 1.2 & 16.8 & 103 & 88. & & \\
\hline & 0.90 & 1.18 & 11.1 & 29.7 & 720 & 15.5 & 71.0 & $<0.002$ & 0.04 & 1.06 & 1 & 1.90 & 206.0 & \begin{tabular}{|l|}
0.76 \\
\end{tabular} & $<0.05$ & 9.9 & 0.416 & 0.41 & 2.1 & 114 & 1.1 & 16.5 & 91 & 80.9 & & 7730 \\
\hline 24 & 0.83 & 1.31 & 10.8 & 26.9 & 740 & 13.1 & 62.2 & $<0.002$ & 0.04 & 1.07 & 1 & 1.60 & 216.0 & \begin{tabular}{|l|}
0.78 \\
\end{tabular} & $<0.05$ & 8.8 & 0.435 & 0.38 & 2.0 & 1 & 1.2 & 15.7 & 87 & 76.3 & & FA04 \\
\hline & & 90 & 10.5 & 28.5 & 770 & 12.6 & 59.5 & $<0.00$ & 0.03 & 1.15 & & & 216.0 & \begin{tabular}{|l|}
0.74 \\
\end{tabular} & $<0.05$ & 8.2 & & 0.39 & 2.2 & 120 & & 16.0 & 208 & 75.7 & & \\
\hline 24 & 0.74 & 1.37 & 10.0 & 27.0 & 700 & 11.9 & 60.4 & $<0.002$ & 0.03 & 1.05 & & 1.50 & 219.0 & \begin{tabular}{|l|}
0.68 \\
\end{tabular} & $<0.05$ & 7.9 & 0.380 & 0.40 & 1.9 & 109 & 1.1 & 14.2 & 90 & 69.2 & & FA040? \\
\hline 243 & 0.72 & 1.44 & 10.4 & 27.1 & 730 & 12.0 & 59.3 & $<0.002$ & 0.02 & 1.03 & $<1$ & 1.60 & 229.0 & \begin{tabular}{|l|}
0.73 \\
\end{tabular} & $<0.05$ & 7.8 & 0.392 & 0.40 & 2.0 & 111 & 1.0 & 14.9 & 101 & 70.0 & & FA040 \\
\hline & & 1.36 & & 28.5 & 760 & 12.2 & & $<0.002$ & 0.03 & 1.06 & & & 229.0 & 0.68 & $<0.05$ & 8.4 & & 0.39 & 2.0 & & & & & 73.3 & & \\
\hline 245 & 0.84 & 1.37 & 11.4 & 29.1 & 810 & 11.0 & 57.5 & $<0.002$ & 0.03 & 1.00 & 1 & 1.50 & 232.0 & 0.83 & $<0.05$ & 8.4 & 0.410 & 0.36 & 2.1 & 107 & 1.1 & 16.8 & 94 & 79.8 & 67 & FA040 \\
\hline 246 & 0.90 & 1.45 & 11.3 & 28.4 & 860 & 11.1 & 56.7 & $<0.002$ & 0.03 & 0.98 & $<1$ & 1.60 & 245.0 & 0.77 & $<0.05$ & 9.1 & 0.449 & 0.36 & 2.1 & 116 & 1.5 & 16.2 & 88 & 88.1 & & \\
\hline & 0.89 & 1.46 & 10.6 & 29.9 & 870 & 12.6 & 60.1 & $<0.002$ & 0.03 & 1.09 & 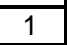 & & 244.0 & 0.73 & $<0.05$ & 9.1 & 0.455 & 0.39 & 2.4 & & & & 122 & 84.1 & & $\mathrm{FAO}$ \\
\hline 248 & 0.91 & 1.35 & 10.9 & 28.7 & 800 & 11.9 & 57.2 & $<0.002$ & 0.03 & 1.06 & 1 & 1.50 & 224.0 & 0.73 & $<0.05$ & 8.8 & 0.418 & 0.37 & 2.0 & 114 & 1.3 & 15.5 & 100 & 76.9 & 40 & 730 \\
\hline & 0.92 & 1.50 & 10.9 & 29.1 & 870 & 12.1 & 59.0 & $<0.002$ & 0.03 & 1.11 & $<1$ & 1.60 & 251.0 & 0.78 & $<0.05$ & 9.2 & 0.455 & 0.38 & 2.1 & & 1.9 & 16.2 & 166 & 78.3 & & \\
\hline 250 & 0.86 & 1.42 & 11.0 & 28.5 & 820 & 11.7 & 58.4 & $<0.002$ & 0.03 & 1.05 & 1 & 1.60 & 236.0 & \begin{tabular}{l|l|}
0.79 \\
\end{tabular} & $<0.05$ & 9.3 & 0.425 & 0.37 & 2.2 & 115 & 1.0 & 16.0 & 88 & 75.2 & 30 & FA04030730 \\
\hline 251 & 0.94 & 1.39 & 10.2 & 29.1 & 840 & 11.7 & 58.6 & $<0.002$ & 0.03 & 1.07 & 1 & 1.60 & 234.0 & 0.71 & $<0.05$ & 9.0 & 0.431 & 0.31 & 2.1 & 120 & 1.1 & 16.0 & 110 & 78.9 & 44 & FA04030730 \\
\hline
\end{tabular}


Table 1. Location and New Trace Element Geochemical Results for 902 Stream-Sediment Samples collected in the Livengood Area, Livengood Quadrangle, Alaska IS = Insufficient Sample for Analysis

\begin{tabular}{|c|c|c|c|c|c|c|c|c|c|c|c|c|c|c|c|c|c|c|c|c|c|c|c|c|c|c|c|}
\hline MPLE & TM E & TM N & $\begin{array}{l}\mathrm{Au} \\
\mathrm{ppm}\end{array}$ & $\begin{array}{c}\mathrm{Ag} \\
\mathrm{ppm}\end{array}$ & $\begin{array}{l}\text { Al } \\
\%\end{array}$ & $\begin{array}{l}\text { As } \\
\text { ppm }\end{array}$ & $\begin{array}{l}\mathrm{Ba}^{*} \\
\mathrm{ppm}\end{array}$ & \begin{tabular}{|c|}
$\mathrm{Be}$ \\
$\mathrm{ppm}$ \\
\end{tabular} & $\begin{array}{c}\mathrm{Bi} \\
\mathrm{ppm}\end{array}$ & $\begin{array}{c}\mathrm{Ca} \\
\%\end{array}$ & $\begin{array}{c}\mathrm{Cd} \\
\mathrm{ppm}\end{array}$ & $\begin{array}{c}\mathrm{Ce} \\
\mathrm{ppm}\end{array}$ & $\begin{array}{l}\text { Co } \\
\text { ppm }\end{array}$ & $\begin{array}{l}\mathrm{Cr}^{\star} \\
\mathrm{ppm}\end{array}$ & $\begin{array}{l}\text { Cs } \\
\text { ppm }\end{array}$ & $\begin{array}{c}\mathrm{Cu} \\
\mathrm{ppm}\end{array}$ & $\begin{array}{c}\mathrm{Fe} \\
\% \\
\end{array}$ & $\begin{array}{c}\mathrm{Ga} \\
\mathrm{ppm}\end{array}$ & $\begin{array}{c}\mathrm{Ge} \\
\mathrm{ppm}\end{array}$ & $\begin{array}{c}\mathrm{Hf} \\
\mathrm{ppm}\end{array}$ & $\begin{array}{l}\mathrm{Hg} \\
\mathrm{ppm}\end{array}$ & $\begin{array}{c}\text { In } \\
\text { ppm }\end{array}$ & $\begin{array}{l}\mathrm{K} \\
\% \\
\end{array}$ & $\begin{array}{r}\mathrm{La} \\
\mathrm{ppm}\end{array}$ & $\begin{array}{c}\mathrm{Li} \\
\mathrm{ppm} \\
\end{array}$ & $\begin{array}{c}\mathrm{Mg} \\
\% \\
\end{array}$ & $\begin{array}{l}\mathrm{Mn} \\
\mathrm{ppm}\end{array}$ \\
\hline 252 & 282 & 0733 & 0.005 & & \begin{tabular}{|l|l}
6.00 \\
\end{tabular} & 8.2 & 890 & & & & \begin{tabular}{|l|l|}
0.32 \\
\end{tabular} & & 112 & 88 & 2.33 & 19.0 & 3.06 & 13.60 & 0.25 & \begin{tabular}{|l|}
2.0 \\
\end{tabular} & 0.08 & 0.044 & \begin{tabular}{|l}
1.32 \\
\end{tabular} & 31.3 & 23.5 & 1.04 & 545 \\
\hline 54 & & & 005 & & \begin{tabular}{|l}
6.13 \\
\end{tabular} & 9.4 & 840 & & & & & & 3 & 8 & 2.5 & 22.5 & 3.18 & 14.90 & 0.25 & 1.9 & 0.15 & 0.044 & \begin{tabular}{|l|}
1.30 \\
\end{tabular} & 32.4 & 4.8 & 1.12 & 717 \\
\hline & & & 0.007 & 11 & 5.94 & 9.0 & 810 & .42 & 0.14 & 81 & \begin{tabular}{|l|}
0.29 \\
\end{tabular} & 8.8 & 13.2 & & 2.50 & 20.4 & 3.16 & 14.70 & 0.26 & 2.0 & 0.28 & .044 & \begin{tabular}{|l|l}
1.27 \\
\end{tabular} & 33.7 & 2.3 & 1.10 & 331 \\
\hline & & & & & & 10.1 & 70 & & & & & & & & & & & & 0.25 & & 0.10 & & 1.38 & & 30.9 & 1.20 & \\
\hline & & & .013 & 0.15 & 6.21 & 13.9 & 920 & 1.36 & 0.14 & 1.82 & \begin{tabular}{|l|l}
0.38 \\
\end{tabular} & 61.6 & 13.9 & 88 & 2.69 & 20.6 & 3.97 & 14.50 & 0.24 & 1.8 & 0.20 & 0.043 & \begin{tabular}{|l|}
1.36 \\
\end{tabular} & 29.9 & 28.1 & 1.12 & 1160 \\
\hline & & & .005 & 0.11 & \begin{tabular}{|l|l|}
6.18 \\
\end{tabular} & 8.6 & 880 & 36 & 0.13 & 1.76 & \begin{tabular}{|l|}
0.31 \\
\end{tabular} & 59.0 & 12.6 & 85 & 2.53 & 18.8 & 3.20 & 14.15 & 0.23 & 1.8 & 0.11 & 0.039 & 1.34 & 28.7 & 24.2 & 1.10 & 1100 \\
\hline & & & & & \begin{tabular}{|l|l|}
6.16 \\
\end{tabular} & 10.2 & 880 & & & 1.80 & & & & & 2.59 & & 3.41 & 14.25 & 0.24 & 18 & & & \begin{tabular}{|l|}
1.34 \\
\end{tabular} & 30.3 & & 1.10 & \\
\hline & & & 0.008 & 0.11 & \begin{tabular}{|l|l|}
5.67 \\
\end{tabular} & 8.4 & 780 & 1.32 & 0.12 & 1.99 & 0.22 & 66.8 & 12.7 & 8 & 2.37 & 20.5 & 2.94 & 13.85 & 0.25 & 2.0 & 0.11 & 0.041 & \begin{tabular}{|l}
1.27 \\
\end{tabular} & 32.6 & 25.7 & 1.06 & 567 \\
\hline & & & .005 & 10 & 5.81 & 7.2 & 800 & 1.14 & 0.11 & 2.31 & 0.22 & 67.9 & 11. & & 2.11 & 18.4 & 3.07 & 13.05 & 0.23 & 2.0 & 0.26 & 0.040 & \begin{tabular}{|l}
1.19 \\
\end{tabular} & 32.8 & 24.5 & 1.12 & 598 \\
\hline & & & .014 & 13 & \begin{tabular}{|l|l}
6.17 \\
\end{tabular} & 9.2 & 830 & 1.43 & 0.13 & 1.77 & \begin{tabular}{|l}
0.23 \\
\end{tabular} & 66.9 & 11. & $\overline{9}$ & 2.61 & 18.0 & 3.07 & 14.60 & 0.27 & 2.0 & 0.13 & 0.041 & 1.28 & 32.3 & 20.3 & 1.08 & 23 \\
\hline & & & .015 & 0.12 & 5.72 & 5.7 & 810 & 1.38 & 0.13 & 1.63 & \begin{tabular}{|l}
0.25 \\
\end{tabular} & 67.2 & 12. & 8 & 2.69 & 21.1 & 2.92 & 14.95 & 0.23 & 1.9 & 0.24 & 0.043 & \begin{tabular}{|l}
1.23 \\
\end{tabular} & 32.1 & \begin{tabular}{|l|}
27.2 \\
\end{tabular} & 1.04 & 438 \\
\hline & & & .005 & 09 & 5.91 & 4. & 760 & .18 & 0.11 & 1.73 & \begin{tabular}{|l}
0.17 \\
\end{tabular} & $\overline{6}$ & 10. & & 2.10 & 15.8 & 2.80 & 13.00 & 0.25 & 1.8 & 0.08 & 0.036 & \begin{tabular}{|l}
1.15 \\
\end{tabular} & 30.1 & 21.8 & 1.02 & 10 \\
\hline & & & 014 & 12 & 37 & 7.3 & 860 & & & 90 & 23 & 9.1 & 11. & & .48 & 18. & 3.29 & 14.30 & 0.25 & $\sqrt{2}$ & 01 & .042 & \begin{tabular}{|l}
1.29 \\
\end{tabular} & 33 & 1.1 & 116 & 99 \\
\hline & & & $<0.005$ & 0.12 & 6.12 & 7.8 & 900 & 1.36 & 0.16 & 1.76 & 0.31 & 62.4 & 13.3 & 8 & 2.72 & 24.5 & 3.17 & 14.70 & 0.26 & 1.9 & 0.15 & 0.040 & 1.44 & 30.4 & 21.6 & 1.09 & 654 \\
\hline & & & .005 & & 5.51 & 5.4 & 1120 & & & 1.09 & & & 11.7 & & $\bar{R}$ & 15.2 & 2.61 & 14.10 & 0.24 & & 0.20 & 039 & \begin{tabular}{|l|}
1.38 \\
\end{tabular} & 37.8 & 38.3 & 0.73 & 334 \\
\hline & & & 0.009 & 38 & 6.66 & 9.0 & 1270 & 1.55 & 0.17 & 1.56 & .64 & 87.7 & 13.4 & & 3.9 & 25. & 3.19 & 15.90 & 0.25 & 2.6 & 0.46 & .046 & 1.78 & 42. & 5.4 & 0.99 & 98 \\
\hline & & & 0.020 & .12 & \begin{tabular}{|l|l|}
5.44 \\
\end{tabular} & 9.5 & 740 & .20 & .16 & 1.06 & 0.22 & 1.8 & 8.1 & & 2.34 & 16. & 2.61 & 12.75 & 0.22 & 1. & 0.20 & 038 & \begin{tabular}{|l|l|}
1.12 \\
\end{tabular} & 26.0 & 16.2 & 0.72 & 290 \\
\hline & & & 0.020 & $\overline{09}$ & 5.31 & & 720 & & & $\overline{32}$ & & & & & & & 2.94 & 1.85 & & & 0.18 & $\overline{036}$ & 1.04 & 29.7 & 7.4 & 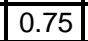 & \\
\hline & & & 0.024 & 0.07 & \begin{tabular}{|l|}
4.99 \\
\end{tabular} & 10.6 & 710 & 1.06 & 0.13 & 1.30 & 0.19 & 59.9 & 10.5 & 8 & 1.96 & 15.6 & 2.66 & 12.80 & \begin{tabular}{|l}
0.22 \\
\end{tabular} & 1.8 & 0.14 & $\overline{041}$ & 1.02 & 29.1 & 9.0 & 0.75 & 448 \\
\hline & & & 520 & & 5.73 & 6.0 & 810 & & & 50 & & & 9. & & 2.0 & 18. & 2.59 & 13.40 & \begin{tabular}{|l}
0.23 \\
\end{tabular} & 1. & 0.5 & 42 & \begin{tabular}{|l}
1.17 \\
\end{tabular} & 29.7 & 20.4 & 0.89 & 353 \\
\hline & & & 0.028 & 10 & \begin{tabular}{|l|l|}
5.15 \\
\end{tabular} & 15.4 & 730 & & & 35 & & & 9.5 & & 1.8 & 16.2 & 3.41 & 2.15 & 0.26 & & 0.26 & & & & 4.8 & & 41 \\
\hline & & & 0.037 & 0.11 & 5.43 & 9.2 & 810 & 1.40 & 0.16 & 1.26 & 0.29 & 56.9 & 13.4 & & 2.56 & 20.6 & 2.78 & 14.70 & 0.23 & 1.7 & 0.30 & .043 & \begin{tabular}{|l|}
1.22 \\
\end{tabular} & 27.6 & 26.0 & 0.83 & 492 \\
\hline & & & 027 & 15 & 5.75 & 5. & 650 & & & 2.48 & 39 & & IU. & & 3.5 & 29. & 3.15 & 3.35 & $0.2 \varepsilon$ & 1. & 0.20 & .044 & 1.08 & 29.0 & 25.3 & 1.01 & 674 \\
\hline & & & & & 4. & 3. & 470 & & & 70 & & & & & & & & & & & & & & 23.3 & \begin{tabular}{|l|}
23.7 \\
\end{tabular} & 0.91 & 79 \\
\hline & & & 0.005 & 20 & 5.61 & 5. & 710 & 1. & 0.09 & 1.38 & 0.58 & & & 20 & 3.9 & 19. & 3.00 & 14.05 & 0.24 & 2. & 1.06 & .041 & \begin{tabular}{|l|l}
1.19 \\
\end{tabular} & 31.0 & 26.0 & \begin{tabular}{|l}
1.67 \\
\end{tabular} & 427 \\
\hline & & & & 28 & & 12.4 & 60 & & & 1.29 & & & 1 & & & & 3.38 & 5.05 & 0.25 & &. .5 & 051 & 1.2 & 8.9 & 33.5 & 0.93 & 741 \\
\hline & & & 022 & 37 & 6.38 & 9.5 & 1400 & 1.50 & 0. & 1.17 & \begin{tabular}{|l}
0.47 \\
\end{tabular} & 61.2 & & & 6.30 & 31. & 3.04 & 15.90 & 0.24 & 2.1 & 1.19 & 047 & \begin{tabular}{|l|l|}
1.43 \\
\end{tabular} & 30.2 & 32.8 & 0.95 & 353 \\
\hline & & & 075 & & 5.87 & 16.8 & 970 & & & 1.42 & 54 & & 20. & & 4.3 & 30. & 4.35 & 5.10 & 0.2 & 2.0 & 0.67 & .049 & \begin{tabular}{|l|}
1.37 \\
\end{tabular} & 25.7 & 28.4 & 1.04 & 3210 \\
\hline & & & & & 5.81 & 8. & 860 & & & 1.34 & & & 17 & & & & 3.3 & 4.15 & 0.2 & & & & & 26 & \begin{tabular}{|l|}
33.4 \\
\end{tabular} & 1.12 & 1360 \\
\hline & & & & & & & 720 & & & & & & & & & & 20 & 3,30 & $\overline{02}$ & & $\sqrt{25}$ & $\overline{0}$ & $\overline{1.2}$ & 22 & & 0.96 & $109 \mathrm{C}$ \\
\hline & & & 0.186 & 29 & 5.09 & 5. & 750 & & & 1.28 & .43 & 55. & 20 & & 4.3 & 23. & 3.05 & 1.85 & 0.22 & 1. & 0.88 & .045 & 1.02 & 24.8 & 22.6 & 0.86 & 1660 \\
\hline & & & & & 6.06 & 5 & 730 & & & 1.26 & & 60 & 15 & & & & 3.17 & & & 1.7 & 0.44 & & 1.22 & 28.5 & 30.9 & 1.10 & 788 \\
\hline & & & 0 & & 5.95 & 64.6 & 680 & & & & & & & & 3. & 320 & 3.34 & .00 & & & 0.2 & .059 & 1.28 & 33.9 & 9.8 & 1.06 & $\overline{421}$ \\
\hline & 520 & 55895 & 0.014 & 0.21 & 5.73 & 65.4 & 630 & 1.28 & 2.70 & 1.12 & 0.23 & 78.3 & 11. & & 3.74 & 134.5 & 3.22 & 14.35 & 0.24 & 1.6 & 0.13 & .066 & 1.24 & 38.1 & 22.8 & 0.98 & 413 \\
\hline & & & & & & & 690 & & & & & & & & & & & & & & & & 1.22 & & $4 . c$ & 0.93 & 60 \\
\hline & & & 0 & & & 74. & 730 & & & 25 & 0.2 & 108.0 & & & 5.7 & 03.5 & 3.79 & 5.20 & 0 & 1 . & 0.1 & .079 & \begin{tabular}{|l|}
1.38 \\
\end{tabular} & 56.3 & 23.4 & 1.06 & 726 \\
\hline & & $24 \pi$ & 0.030 & 0.31 & 6.01 & 5.2 & 810 & 1.2 & 0. & 1.54 & \begin{tabular}{|l}
0.76 \\
\end{tabular} & 66.4 & 10. & 103 & 8.3 & 22. & 2.70 & 13.65 & 0.23 & 1.6 & .23 & .044 & \begin{tabular}{|l|}
1.18 \\
\end{tabular} & 31.8 & 49.3 & 0.90 & 919 \\
\hline & & & & & & & & & & & & & & & & & & & & & 2 & & \begin{tabular}{|l|l|}
1.14 \\
\end{tabular} & 255 & 0 & 80 & 66 \\
\hline & & & 013 & 0.24 & 6.14 & 5.7 & 730 & & 0. & 2.11 & \begin{tabular}{|l}
0.56 \\
\end{tabular} & & 11 & & 8. & & 3.01 & 13.70 & 0.24 & 1.5 & 0.23 & 0.044 & \begin{tabular}{|l|}
1.26 \\
\end{tabular} & 30.4 & 0.9 & \begin{tabular}{|l|l|}
1.04 \\
\end{tabular} & 1125 \\
\hline & & & & 0.26 & 6.69 & 8.9 & 820 & 1. & 0. & 1.37 & \begin{tabular}{|l|l|} 
\\
\end{tabular} & 58 & 13. & 100 & 3.52 & 28 & 3.28 & 15.30 & 0.25 & 1.7 & 0.10 & 0.051 & 1.30 & 29.7 & 27.7 & 1.09 & 506 \\
\hline & & & & & & 4.9 & 760 & & & & & & & 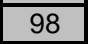 & & & 2.88 & & & 1. &.$\perp$ & & \begin{tabular}{|l|}
1.21 \\
\end{tabular} & 30.8 & 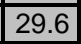 & 1.08 & 771 \\
\hline & & 7252 & 0.00 & 0.16 & 6.28 & $\begin{array}{l}7.9 \\
\end{array}$ & 770 & 1.22 & 0.43 & 1.58 & 0.30 & 68. & 14. & 101 & 3.4 & 26. & 3.3 & 14.95 & 0. & 1.7 & 0.14 & 05 & 1.23 & 32.3 & 32.5 & 1.16 & 29 \\
\hline & & & 0.015 & 0.18 & 10.0 & 7.5 & 790 & & 0.41 & & & 68.5 & 13.1 & 100 & 0.00 & ¿2..0 & 3.100 & & & & 0.12 & 0.045 & 1.23 & 32.4 & & 1.08 & 92 \\
\hline
\end{tabular}


Table 1. Location and New Trace Element Geochemical Results for 902 Stream-Sediment Samples collected in the Livengood Area, Livengood Quadrangle, Alaska IS = Insufficient Sample for Analysis

\begin{tabular}{|c|c|c|c|c|c|c|c|c|c|c|c|c|c|c|c|c|c|c|c|c|c|c|c|c|c|c|}
\hline SAMPLE & $\begin{array}{l}\text { Mo } \\
\text { ppm }\end{array}$ & $\begin{array}{c}\mathrm{Na} \\
\%\end{array}$ & $\begin{array}{l}\mathrm{Nb} \\
\mathrm{ppm}\end{array}$ & $\begin{array}{c}\mathrm{Ni} \\
\mathrm{ppm}\end{array}$ & $\begin{array}{c}\mathrm{P} \\
\mathrm{ppm}\end{array}$ & $\begin{array}{l}\mathrm{Pb} \\
\mathrm{ppm}\end{array}$ & $\begin{array}{l}\mathrm{Rb} \\
\mathrm{ppm}\end{array}$ & $\begin{array}{l}\mathrm{Re} \\
\mathrm{ppm}\end{array}$ & $\begin{array}{l}\mathrm{S} \\
\%\end{array}$ & $\begin{array}{l}\text { Sb } \\
\text { ppm }\end{array}$ & $\begin{array}{c}\text { Se } \\
\text { ppm }\end{array}$ & $\begin{array}{l}S^{S} n^{*} \\
\text { ppm }\end{array}$ & $\begin{array}{c}\mathrm{Sr} \\
\mathrm{ppm}\end{array}$ & $\begin{array}{l}\mathrm{Ta}^{*} \\
\mathrm{ppm}\end{array}$ & $\begin{array}{c}\text { Te } \\
\text { ppm }\end{array}$ & $\begin{array}{c}\text { Th } \\
\text { ppm }\end{array}$ & $\begin{array}{l}\mathrm{Ti}^{*} \\
\%\end{array}$ & $\begin{array}{c}\mathrm{Tl} \\
\mathrm{ppm}\end{array}$ & $\begin{array}{c}U \\
\mathrm{ppm}\end{array}$ & $\begin{array}{c}\mathrm{V} \\
\mathrm{ppm}\end{array}$ & $\begin{array}{l}W^{*} \\
\mathrm{ppm}\end{array}$ & \begin{tabular}{|c|}
$\mathrm{Y}$ \\
$\mathrm{ppm}$
\end{tabular} & $\begin{array}{l}\mathrm{Zn} \\
\mathrm{ppm}\end{array}$ & $\begin{array}{l}\mathrm{Zr}^{*} \\
\mathrm{ppm}\end{array}$ & $\begin{array}{c}\text { Pulp Wt } \\
\text { grams }\end{array}$ & $\begin{array}{c}\text { Lab } \\
\text { Report }\end{array}$ \\
\hline 252 & 0.88 & 1.43 & 11.2 & 28.2 & 860 & 11.1 & 56.7 & \begin{tabular}{|c|}
$<0.002$ \\
\end{tabular} & 0.03 & 1.03 & 1 & 1.50 & 238.0 & 0.76 & $<0.05$ & 8.6 & 0.452 & 0.35 & 2.1 & \begin{tabular}{|l|}
119 \\
\end{tabular} & 1.1 & 15.4 & 103 & 74.2 & 55 & FA04030730 \\
\hline 254 & 0.66 & 1.39 & 10.8 & 34.7 & 840 & 12.1 & 61.8 & $\begin{array}{l}<0.002 \\
\end{array}$ & 0.04 & 1.17 & 1 & & 254.0 & 0.76 & $<0.05$ & 8.8 & 0.426 & 0.38 & 2.2 & 115 & 1.0 & 16.2 & 108 & 70.7 & 35 & $A 04030730$ \\
\hline 255 & .64 & .35 & 11.5 & 34.3 & 820 & 11.6 & 61.2 & $<0.002$ & 0.04 & 1.15 & $<1$ & 1.70 & 249.0 & 0.84 & $<0.05$ & 9.0 & 0.448 & 0.38 & 2.2 & 117 & 1.1 & 16.2 & 86 & 77.5 & 44 & $=\mathrm{A} 04030730$ \\
\hline 256 & 0.57 & 1.54 & 11.4 & 32.8 & 890 & 11.4 & 62.6 & $<0.002$ & 0.04 & 1.01 & & 1.60 & 281.0 & 0.79 & $<0.05$ & 10.1 & 0.497 & 0.39 & 2.3 & 126 & 1.2 & 16.4 & 106 & 77.1 & 4 & FA04030730 \\
\hline 257 & 67 & 36 & 10.6 & 33.3 & 960 & 12.4 & 64.1 & \begin{tabular}{l|}
$<0.002$ \\
\end{tabular} & 0.08 & 1.12 & 1 & 1.60 & 259.0 & 0.72 & $<0.05$ & 8.7 & 0.421 & 0.40 & 2.2 & 122 & 1.1 & \begin{tabular}{|l|l|}
15.4 & \\
\end{tabular} & 101 & 67.7 & & \\
\hline 258 & 0.66 & 1.37 & 10.1 & 31.3 & 820 & 11.4 & 62.3 & $<0.002$ & 0.05 & 1.02 & 1 & 1.60 & 249.0 & 0.70 & $<0.05$ & 8.0 & 0.416 & 0.41 & 2.1 & 117 & 1.0 & 14.0 & 99 & 67.1 & & FA04030730 \\
\hline 259 & 0.67 & 1.33 & 10.4 & 32.6 & 860 & 11.9 & 63.2 & $<0.002$ & 0.05 & 1.08 & $<1$ & 1.70 & 253.0 & 0.72 & $<0.05$ & 8.4 & 0.438 & 0.41 & 2.2 & 121 & 1.0 & 15.0 & 102 & 68.3 & 2 & FA04030730 \\
\hline 260 & .70 & 1.37 & \begin{tabular}{|l|l}
10.7 \\
\end{tabular} & 32.0 & 740 & 11.5 & 56.3 & $<0.002$ & 0.02 & 1.25 & $<1$ & 1.50 & 268.0 & 0.77 & $<0.05$ & 8.8 & 0.422 & 0.36 & 1.9 & 110 & 1.2 & \begin{tabular}{|l|}
15.2 \\
\end{tabular} & 77 & 75.2 & & FA04030730 \\
\hline 261 & 0.64 & 1.39 & 10.4 & 30.4 & 760 & 10.6 & 51.7 & $<0.002$ & 0.05 & 1.02 & $<1$ & 1.40 & 287.0 & 0.75 & $<0.05$ & 8.8 & 0.462 & 0.34 & 2.0 & 115 & 1.0 & 15.3 & 81 & 73.4 & 6 & FA04030730 \\
\hline 2 & & 36 & 10.8 & 31.2 & 750 & 11.0 & 61.2 & $<0.002$ & 0.03 & 1.12 & & 1.70 & 220.0 & 0.74 & $<0.05$ & 8.9 & 0.458 & 0.41 & 2.1 & 120 & 1.2 & 15.9 & & 78.3 & & FA04030730 \\
\hline 33 & .66 & 1.32 & 11.0 & 33.0 & 700 & 11.8 & 61.9 & $<0.002$ & 0.03 & 1.06 & 1 & 1.80 & 209.0 & 0.74 & $<0.05$ & 9.0 & 0.421 & 0.41 & 2.1 & 115 & 1.1 & 16.2 & 87 & 71.4 & & FA04 \\
\hline 264 & 0.46 & 1.48 & 10.2 & 26.4 & 640 & 10.9 & 51.2 & $<0.002$ & 0.02 & 0.88 & $<1$ & 1.50 & 217.0 & 0.72 & $<0.05$ & 8.0 & 0.472 & 0.35 & 2.0 & 114 & 1.0 & 14.7 & 75 & 66.0 & & FA04030730 \\
\hline & 0.66 & 1.44 & 11.0 & 30.5 & 820 & 11.9 & 60.3 & $<0.002$ & 0.03 & 1.02 & & 1.70 & 231.0 & 0.73 & $<0.05$ & 9.5 & 0.491 & 0.38 & 2.2 & 128 & 1.1 & \begin{tabular}{|l|}
15.4 \\
\end{tabular} & 94 & 77.6 & & \\
\hline 266 & 0.74 & 1.30 & 10.6 & 35.5 & 790 & 12.6 & 66.2 & $<0.002$ & 0.03 & 1.17 & $<1$ & 1.70 & 214.0 & 0.75 & $<0.05$ & 8.8 & 0.451 & 0.44 & 2.0 & 123 & 1.1 & \begin{tabular}{|l|}
15.8 \\
\end{tabular} & 97 & 70.6 & & 30730 \\
\hline 323 & 1.09 & 0.91 & 11.2 & 28.2 & 680 & 11.4 & 72.9 & $<0.002$ & 0.05 & 0.94 & & 1.70 & 139.0 & 0.80 & $<0.05$ & 11.4 & 0.436 & 0.49 & 2.7 & 102 & 1.2 & 15.2 & 136 & 89.1 & 2 & FA04030730 \\
\hline 3 & 64 & 0.87 & 11.6 & 40.5 & 1070 & 13.5 & 87.0 & 0.002 & 0.09 & 1.92 & 2 & 1.90 & 156.5 & 0.82 & $<0.05$ & 12.4 & 0.529 & 0.67 & 3.0 & 131 & 1.4 & 17.6 & 135 & 101.5 & & \\
\hline 351 & 0.98 & 1.15 & 9.5 & 21.6 & 570 & 12.5 & 54.3 & $<0.002$ & 0.03 & 1.27 & $<1$ & 3.10 & 172.5 & 0.64 & $<0.05$ & 7.3 & 0.410 & 0.39 & 2.2 & 117 & 1.0 & \begin{tabular}{|l|}
11.6 \\
\end{tabular} & 60 & 62.8 & & FA04 \\
\hline 3 & 0.95 & 1.23 & 9.5 & 20.9 & 640 & 11.7 & 47.8 & $<0.002$ & 0.03 & 1.15 & $<1$ & 1.90 & 190.0 & 0.66 & $<0.05$ & 8.2 & 0.427 & 0.33 & 2.1 & 112 & $0 .{ }^{2}$ & 12.8 & 5 & 67.2 & & 730 \\
\hline & 0.85 & 1.21 & 9.7 & 23.2 & 580 & 11.7 & 52.8 & $<0.002$ & 0.02 & 1.17 & $<1$ & 3.20 & 186.5 & 0.72 & $<0.05$ & 7.8 & 0.395 & 0.32 & 2.2 & 102 & 1.0 & 14.5 & 56 & 64.6 & & 0730 \\
\hline 354 & 0.55 & 1.40 & 10.0 & 25.1 & 590 & 12.4 & 53.5 & $<0.002$ & 0.02 & 1.17 & 1 & 2.00 & 215.0 & 0.75 & $<0.05$ & 8.3 & 0.434 & 0.34 & 2.3 & 113 & 1.1 & 14.8 & 67 & 70.9 & 3 & FA04030730 \\
\hline 3 & 1.03 & 1.23 & \begin{tabular}{|l|}
8.9 \\
\end{tabular} & 23.3 & 860 & 10.7 & 50.7 & $<0.002$ & 0.06 & 1.04 & $<1$ & 1.70 & 190.5 & 0.67 & $<0.05$ & 7.6 & 0.387 & 0.34 & 2.1 & 102 & 0.6 & 13.8 & 59 & 62.4 & & 0730 \\
\hline & .83 & 1.23 & 9.7 & 26.8 & 660 & 13.3 & 65.7 & 0.005 & 0.03 & 1.21 & & 1.90 & 191.5 & 0.68 & $<0.05$ & 8.1 & 0.365 & 0.42 & 2.2 & 10 & 1. & 14.2 & 72 & 64.4 & & 730 \\
\hline 3 & 0.66 & 0.96 & 7.6 & 32.6 & 1080 & 12.0 & 67.5 & $<0.002$ & 0.10 & 0.98 & & 1.80 & 175.0 & 0.55 & $<0.05$ & 7.3 & 0.374 & 0.37 & 2.2 & 107 & 0. & 21.3 & 96 & 60.0 & & FA04030730 \\
\hline & 0.43 & 0.93 & 6.4 & 27.9 & 820 & 12.5 & 54.0 & $<0.002$ & 0.11 & 0.87 & & 1.80 & 162.5 & 0.47 & $<0.05$ & 5.8 & 0.327 & 0.29 & 1.7 & 8 & 0.7 & 15.4 & 74 & 53.1 & & 5730 \\
\hline 3 & .02 & 1.09 & 14.6 & 94.8 & 630 & 9.5 & 66.9 & $<0.002$ & 0.10 & 1.77 & & 2.60 & 148.5 & 1.06 & $<0.05$ & 8.1 & 0.389 & 0.63 & 2.0 & 161 & 0.9 & 16.8 . & 156 & 121.5 & & 0730 \\
\hline 3 & 1.91 & 0.73 & 10.2 & 115.0 & 670 & 14.4 & 84.2 & \begin{tabular}{|l|}
0.002 \\
\end{tabular} & 0.06 & 2.09 & & 2.70 & 160.0 & \begin{tabular}{|l|l|}
0.74 \\
\end{tabular} & $<0.05$ & 8.4 & 0.367 & 0.77 & 2.5 & 15 & 1.1 & 16.0 & 109 & 89.0 & & 0730 \\
\hline 364 & 1.53 & 0.75 & 10.2 & 66.2 & 730 & 14.0 & 85.5 & 0.003 & 0.06 & 2.00 & 2 & 2.10 & 154.0 & 0.71 & \begin{tabular}{|l|}
0.06 \\
\end{tabular} & 8.7 & 0.413 & 0.84 & 2.9 & 1 & & 16.6 & 104 & 79 & & 0730 \\
\hline & 1.31 & 0.90 & 9.1 & 63.9 & 870 & 13.6 & 71.6 & $<0.002$ & 0.07 & 1.40 & & 1.90 & 167.0 & 0.62 & 0.06 & 7.2 & 0.386 & 0.49 & 2.2 & 12 & & \begin{tabular}{|l|}
17.1 \\
\end{tabular} & 126 & 76. & & 30730 \\
\hline 3 & 0.85 & 1.15 & 8.7 & 51.5 & 790 & 12.3 & 62.7 & $<0.002$ & 0.06 & 1.18 & & 1.70 & 167.5 & 0.62 & 0.05 & 7.3 & 0.398 & 0.43 & 1.8 & 14 & 1. & 15.3 & 138 & 65.9 & & 0730 \\
\hline & 0.93 & 0.83 & 8.1 & 55.7 & 900 & 12.9 & 65.8 & $<0.002$ & 0.08 & 1.21 & & 2.1 & 155.0 & 0.52 & 0.05 & 6.6 & 0.363 & 0.45 & 2.0 & & & \begin{tabular}{|l|l|}
13.4 \\
\end{tabular} & 122 & 68. & & \\
\hline 374 & 0.65 & 0.85 & 7.1 & 31.2 & 1120 & 12.5 & 58.1 & $<0.002$ & 0.11 & 1.15 & & 1.80 & 153.5 & 0.51 & $<0.05$ & 6.0 & 0.347 & 0.39 & 1.6 & 10 & 0. & \begin{tabular}{|l|}
16.4 \\
\end{tabular} & 83 & 48.8 & & 7730 \\
\hline 375 & 0.56 & 1.20 & 9.7 & 35.3 & 730 & 13.4 & 65.0 & $<0.002$ & 0.07 & 1.13 & 1 & 2.00 & 166.0 & 0.68 & $<0.05$ & 7.5 & 0.449 & 0.42 & 1.9 & 12 & $\overline{1}$ & \begin{tabular}{|l|}
14.8 \\
\end{tabular} & 118 & 60.2 & 2 & 0730 \\
\hline & 0.82 & 1.27 & 10.4 & 32.1 & 650 & & 75.4 & & 0.06 & 1.79 & & 3.80 & 185.5 & 0.77 & \begin{tabular}{|l|}
0.17 \\
\end{tabular} & 8.5 & & 0.62 & 2.1 & & & \begin{tabular}{|l|l|}
13.7 \\
\end{tabular} & 156 & & & \\
\hline 389 & 0.85 & 1.14 & 10.6 & 33.7 & 640 & 13.8 & 83.7 & $<0.002$ & 0.04 & 1.49 & & 5.10 & 158.0 & 0.72 & 0.15 & 8.9 & 0.480 & 0.63 & 1.9 & 119 & 144.0 & 13.2 & 216 & 59.7 & $\varepsilon$ & FA04030730 \\
\hline 391 & 0.80 & 1.23 & 10.4 & 32.5 & 580 & 12.3 & 74.0 & \begin{tabular}{|c|}
$<0.002$ \\
\end{tabular} & 0.03 & 1.63 & $<1$ & 3.70 & 174.5 & 0.73 & 0.10 & 9.1 & 0.453 & 0.51 & 2.1 & 114 & 32.0 & 13.4 & 281 & 66.1 & & 0730 \\
\hline & & 1.13 & 10.8 & 39.9 & 810 & 16.4 & 85.6 & \begin{tabular}{|l|}
0.004 \\
\end{tabular} & 0.04 & 2.21 & & 5.30 & 167.0 & 0.78 & 0.18 & 9.0 & 0.503 & 0.61 & 2.2 & & 389.0 & 15.2 & 154 & 67.7 & & \\
\hline 394 & 0.54 & 1.06 & \begin{tabular}{|l|}
8.8 \\
\end{tabular} & 39.3 & 850 & 19.1 & 61.2 & $<0.002$ & 0.08 & 1.35 & $\perp$ & 1.60 & 227.0 & 0.63 & $<0.05$ & 8.1 & 0.419 & 0.45 & 2.3 & 117 & \begin{tabular}{|l|}
1.8 \\
\end{tabular} & 16.8 & 283 & 59.5 & 16 & FA04C \\
\hline 395 & 0.41 & 0.98 & 10.0 & 42.6 & 710 & 19.0 & 61.9 & $<0.002$ & 0.10 & 1.26 & 1 & 1.80 & 282.0 & 0.77 & $<0.05$ & 7.6 & 0.457 & 0.43 & 1.8 & 110 & 1. & 17.2 & 534 & 61.3 & & 5730 \\
\hline & 0.37 & 1.10 & \begin{tabular}{|l|}
8.7 \\
\end{tabular} & 39.4 & 760 & 16.8 & 61.0 & $<0.002$ & 01 & 1.17 & & 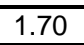 & 271.0 & \begin{tabular}{|l|l|}
0.64 \\
\end{tabular} & $<0.05$ & 7.4 & 0.429 & 0.43 & 1.8 & & & \begin{tabular}{|l|l|}
14.7 \\
\end{tabular} & 157 & 57.3 & & 7730 \\
\hline 400 & 0.84 & 1.41 & 10.1 & 31.3 & 670 & 18.7 & 67.0 & \begin{tabular}{|l|}
$<0.002$ \\
\end{tabular} & 0.04 & 1.71 & $<1$ & 1.80 & 189.0 & 0.70 & 0.05 & 8.7 & 0.480 & 0.45 & 2.2 & 134 & 1. & \begin{tabular}{|l|l|}
13.7 \\
\end{tabular} & 140 & 61.9 & 36 & 730 \\
\hline & 0.59 & 1.34 & 10.2 & 28.9 & 560 & 15.8 & 61.6 & \begin{tabular}{l|l|}
$<002$ \\
\end{tabular} & 0.04 & 1.60 & 1 & 1.80 & 195.5 & 0.72 & $<0.05$ & 8.4 & 0.489 & 0.41 & 2.1 & 12 & & 14.9 & 148 & 60.1 & & \\
\hline 4 & 0.78 & 1.44 & 10.2 & 34.2 & 680 & 14.3 & 63.6 & $<0.002$ & 0.03 & 1.48 & 1 & 1.70 & 201.0 & 0.78 & $<0.05$ & 9.1 & 0.476 & 0.41 & 2.2 & $12 \mathrm{~s}$ & 3.1 & 15.0 & 130 & 62.6 & 32 & FA04030730 \\
\hline 406 & 0.70 & 1.39 & 10.5 & 31.8 & 680 & 14.7 & 62.3 & $<0.002 \mid$ & 0.03 & 1.60 & & 1.80 & 206.0 & 0.78 & 0.05 & 9.0 & 0.461 & 0.42 & 2.3 & 119 & 1.7 & 15.1 & 120 & 66.2 & $\angle 0$ & FA04030730 \\
\hline
\end{tabular}


Table 1. Location and New Trace Element Geochemical Results for 902 Stream-Sediment Samples collected in the Livengood Area, Livengood Quadrangle, Alaska IS = Insufficient Sample for Analysis

\begin{tabular}{|c|c|c|c|c|c|c|c|c|c|c|c|c|c|c|c|c|c|c|c|c|c|c|c|c|c|c|c|}
\hline MPLE & TM E & TM N & $\begin{array}{l}\mathrm{Au} \\
\mathrm{ppm}\end{array}$ & $\begin{array}{c}\mathrm{Ag} \\
\mathrm{ppm}\end{array}$ & $\begin{array}{l}\text { Al } \\
\%\end{array}$ & $\begin{array}{l}\text { As } \\
\text { ppm }\end{array}$ & $\begin{array}{l}\mathrm{Ba}^{*} \\
\mathrm{ppm}\end{array}$ & \begin{tabular}{|c|}
$\mathrm{Be}$ \\
$\mathrm{ppm}$ \\
\end{tabular} & $\begin{array}{c}\mathrm{Bi} \\
\mathrm{ppm}\end{array}$ & $\begin{array}{c}\mathrm{Ca} \\
\%\end{array}$ & $\begin{array}{c}\mathrm{Cd} \\
\mathrm{ppm}\end{array}$ & $\begin{array}{c}\mathrm{Ce} \\
\mathrm{ppm}\end{array}$ & $\begin{array}{l}\text { Co } \\
\text { ppm }\end{array}$ & $\begin{array}{l}\mathrm{Cr}^{*} \\
\mathrm{ppm}\end{array}$ & $\begin{array}{l}\text { Cs } \\
\text { ppm }\end{array}$ & $\begin{array}{c}\mathrm{Cu} \\
\mathrm{ppm}\end{array}$ & $\begin{array}{c}\mathrm{Fe} \\
\% \\
\end{array}$ & $\begin{array}{c}\mathrm{Ga} \\
\mathrm{ppm}\end{array}$ & $\begin{array}{c}\mathrm{Ge} \\
\mathrm{ppm}\end{array}$ & $\begin{array}{c}\mathrm{Hf} \\
\mathrm{ppm}\end{array}$ & $\begin{array}{l}\mathrm{Hg} \\
\mathrm{ppm}\end{array}$ & $\begin{array}{c}\text { In } \\
\text { ppm }\end{array}$ & $\begin{array}{l}\mathrm{K} \\
\% \\
\end{array}$ & $\begin{array}{r}\mathrm{La} \\
\mathrm{ppm}\end{array}$ & $\begin{array}{c}\mathrm{Li} \\
\mathrm{ppm} \\
\end{array}$ & $\begin{array}{c}\mathrm{Mg} \\
\% \\
\end{array}$ & $\begin{array}{l}\mathrm{Mn} \\
\mathrm{ppm}\end{array}$ \\
\hline 434 & 2056 & 61564 & & 0.24 & 5.02 & & 700 & 1.20 & & 0.80 & 0.56 & & 187 & 76 & & 47.5 & 3.09 & 13.55 & 0.22 & \begin{tabular}{|l}
1.9 \\
\end{tabular} & 0.18 & 0.046 & 1.02 & 26.5 & 23.3 & 0.66 & 1155 \\
\hline 64 & & & & & \begin{tabular}{|l|l}
4.67 \\
\end{tabular} & 77.8 & 420 & \begin{tabular}{|l}
1.60 \\
\end{tabular} & 0.32 & & \begin{tabular}{|l|}
0.83 \\
\end{tabular} & 74.4 & 9.6 & & .37 & 8.5 & 2.31 & 13.55 & 0.23 & 2.4 & 0.34 & 0.036 & \begin{tabular}{|l}
1.53 \\
\end{tabular} & 37.5 & 6.6 & \begin{tabular}{|l|l|}
0.53 \\
\end{tabular} & 339 \\
\hline & 424 & & 006 & 12 & 2.92 & 8.2 & 550 & 87 & 0.12 & 76 & .74 & 8.1 & 12.0 & & & 2.8 & 1.56 & 7.43 & 0.25 & 1.2 & 0.22 & .022 & \begin{tabular}{|l|l}
0.73 \\
\end{tabular} & 9.3 & 2.2 & 4.81 & 57 \\
\hline & & & & & 5.82 & & 90 & & & & & & 36 & & & & 3.18 & 14.80 & 0.27 & 19 & 0.47 & & \begin{tabular}{|l|}
1.27 \\
\end{tabular} & 30.6 & & 1.10 & 17 \\
\hline & 547 & 68165 & .005 & 0.11 & \begin{tabular}{|l|l|}
5.74 \\
\end{tabular} & 12.6 & 820 & 1.28 & 0.14 & 1.84 & .33 & 69.2 & 12.2 & 85 & 2.41 & 20.4 & 2.94 & 14.80 & 0.26 & 2.0 & 0.31 & 0.047 & 1.26 & 33.3 & 11.6 & \begin{tabular}{|l|l}
1.04 \\
\end{tabular} & 531 \\
\hline & & & 006 & 11 & 5.44 & 8.4 & 780 & 1.26 & 0.16 & 1.32 & 0.28 & 61.8 & 14.5 & 84 & 2.52 & 17.8 & 2.86 & 14.10 & 0.25 & 1.9 & 0.22 & 0.047 & 1.16 & 29.6 & 21.7 & 0.89 & 331 \\
\hline & & & & & 5.66 & 8. & 860 & 1.20 & & & 0.29 & & 13 & & 233 & $\overline{0.5}$ & $\overline{3.06}$ & 12.95 & 0.26 & 1.8 & 0.24 & & & & & 1.04 & \\
\hline & & & 012 & 10 & 5.71 & 7.2 & 820 & 1.31 & 0.12 & 1.72 & .24 & 75.9 & 11.8 & $\overline{9}$ & 2.23 & 17.1 & 2.97 & 13.75 & 0.30 & 2.1 & 0.32 & 0.044 & 1.18 & 36.7 & 18.2 & 1.04 & 529 \\
\hline & & & & $\overline{09}$ & 5.83 & 5.9 & 870 & 1.18 & 0.13 & 1.83 & 0.23 & 67.9 & 10.5 & & 2.14 & 14.2 & 2.81 & 13.50 & 0.25 & 2.0 & 0.15 & 0.041 & $\begin{array}{l}1.20 \\
\end{array}$ & 32.2 & 21.3 & 1.03 & 481 \\
\hline & & & $\overline{006}$ & 11 & \begin{tabular}{|l}
6.00 \\
\end{tabular} & 5.4 & 900 & 1.26 & 0.1 & 1.97 & \begin{tabular}{|l}
0.23 \\
\end{tabular} & 74.8 & 10.7 & $\overline{0}$ & 2.12 & 4.8 & 2.96 & 13.65 & 0.27 & 2.1 & 0.17 & 0.043 & 1.25 & 36.3 & 20.4 & 1.08 & 521 \\
\hline & & & .007 & 0.07 & 5.87 & 7.3 & 850 & 1.18 & 0.12 & 1.63 & 0.26 & 59.6 & 10. & 79 & 2.05 & 14.7 & 2.80 & 13.25 & 0.16 & 1.8 & 0.18 & .040 & \begin{tabular}{|l}
1.22 \\
\end{tabular} & 28.8 & \begin{tabular}{|l|}
23.6 \\
\end{tabular} & 0.95 & 452 \\
\hline & & & 016 & 17 & \begin{tabular}{|l}
6.29 \\
\end{tabular} & 19.8 & 860 & .44 & 0.21 & 1.00 & \begin{tabular}{|l|}
0.61 \\
\end{tabular} & & 19. & & 5.45 & 4. & 3.29 & 16.15 & 0.13 & 2.6 & 0.39 & .049 & \begin{tabular}{|l|}
1.30 \\
\end{tabular} & 30.1 & 26.4 & 0.82 & 015 \\
\hline & & & 011 & 11 & 5.98 & 6.2 & 810 & 38 & ).14 & .34 & 49 & 4.1 & 16. & & 5 & $\overline{9}$ & 3.17 & 14.60 & 0.15 & $\overline{1.8}$ & 0.28 & .047 & \begin{tabular}{|l|l|}
1.15 \\
\end{tabular} & 9.8 & 5.3 & 0.97 & 906 \\
\hline & & & 010 & 0.10 & \begin{tabular}{|l|l|}
5.60 \\
\end{tabular} & 9.5 & 780 & 1.34 & 0.16 & 1.70 & 0.46 & 59.8 & 17.0 & 92 & 2.38 & 24.1 & 3.35 & 13.70 & 0.18 & 1.8 & 0.19 & 0.040 & 1.04 & 28.4 & 24.2 & 0.95 & 1545 \\
\hline & & & & & \begin{tabular}{|l|l|}
5.97 \\
\end{tabular} & & & & & \begin{tabular}{l|l}
1.65 \\
\end{tabular} & & & 18.7 & & 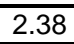 & 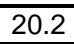 & 3.46 & 14.45 & 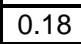 & 1.8 & 0.15 & 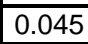 & 1.21 & 31.1 & 26.6 & \begin{tabular}{|l|l|}
1.12 \\
\end{tabular} & 510 \\
\hline & & & 0.005 & 36 & \begin{tabular}{|l|l|}
8.15 \\
\end{tabular} & 13.7 & 1490 & 87 & 0.35 & 0.36 & 0.24 & 81. & 3.5 & 0 & 8.15 & 14. & 2.07 & 2.00 & 0.09 & 2.4 & 0.32 & .062 & \begin{tabular}{|l}
0.96 \\
\end{tabular} & 40.1 & 78.4 & 0.33 & 133 \\
\hline & & & 011 & 35 & \begin{tabular}{|l|l|}
7.60 \\
\end{tabular} & 18.8 & 120 & .60 & .27 & 1.02 & 0.21 & 64.2 & 13. & 10 & 7.61 & 48.2 & 3.68 & 20.70 & 0.14 & 2.0 & 0.53 & .054 & \begin{tabular}{|l}
0.96 \\
\end{tabular} & 30.9 & 70.5 & 0.83 & 563 \\
\hline & & & $\overline{005}$ & $\overline{22}$ & \begin{tabular}{|l|l|}
6.71 \\
\end{tabular} & & 30 & & & 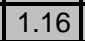 & & & & & & & & 7.85 & 0.1 & & 30 & 54 & 0.90 & 9.3 & 3.8 & 0.83 & $\overline{50}$ \\
\hline & & & .005 & .17 & \begin{tabular}{|l|}
6.57 \\
\end{tabular} & 11.5 & 790 & 1.29 & 0.19 & 1.09 & 0.24 & 60.2 & 11. & 8 & 3.82 & 34.4 & 3.31 & 17.30 & 0.14 & 2.0 & 0.40 & 052 & 1.08 & 28.7 & 38.1 & 0.80 & 438 \\
\hline & & & .005 & & 6.62 & 2.4 & 760 & & & 1.16 & & 7 & 28 & & 3.9 & 32. & 4.19 & 16.70 & 0.1 & 2. & 0.4 & .055 & 1.10 & 32.9 & 61.7 & \begin{tabular}{|l|l|}
0.77 \\
\end{tabular} & 070 \\
\hline & & & 020 & 19 & 6.55 & 14.8 & 790 & 1.58 & 0.16 & 1.28 & \begin{tabular}{|l|}
0.46 \\
\end{tabular} & 76.6 & 16. & & 3.5 & 24.2 & 3.58 & 6.45 & 0.23 & 2.2 & 0.49 & .055 & 1.30 & 36.7 & 1.1 & 0.88 & 688 \\
\hline & & & .005 & 0.64 & 5.78 & 20.8 & 960 & 1.62 & 0.16 & \begin{tabular}{|c|}
0.78 \\
\end{tabular} & \begin{tabular}{|l}
0.75 \\
\end{tabular} & 56.8 & 21. & & 5.69 & 30.7 & 2.57 & 15.60 & 0.13 & 2.2 & 0.42 & .045 & 0.75 & 27.3 & 65.2 & 0.51 & 861 \\
\hline & & & 011 & 51 & 33 & 2.7 & 900 & & & 1.58 & & $5 \%$ & 16. & & 5.7 & 28. & 2.43 & 5.95 & 0.1 & 2.0 & 0.56 & .043 & 0.90 & 29.6 & 89.2 & 0.53 & 758 \\
\hline & & & 005 & & & 22.2 & 830 & & & 0.92 & & & & & & & & & & 2.4 & & & \begin{tabular}{|l|l}
1.42 \\
\end{tabular} & 34.4 & 64.8 & 0.66 & 27 \\
\hline & & & 005 & .23 & 6.56 & 23.9 & 870 & & 0.2 & .09 & 0.54 & 87 & 16. & & & 40.2 & 4.02 & 17.25 & 0.2 & 2. & 0.77 & .071 & 1.44 & 42.1 & 71.0 & 0.72 & 820 \\
\hline & & & 007 & 20 & & 5.1 & & & & 14 & & & & & & & 3.80 & 6.15 & 0.23 & & 8.8 & 062 & 1.37 & 33.7 & 9.9 & 0.74 & 691 \\
\hline & & & 019 & 31 & 6.61 & 11.2 & 810 & 1.60 & 0.20 & 0.94 & 0.43 & 67.4 & 13.6 & 10 & 4.79 & 38.5 & 3.33 & 17.25 & 0.18 & 1.9 & 0.31 & 062 & \begin{tabular}{|l|}
1.39 \\
\end{tabular} & 33.8 & 9.8 & \begin{tabular}{|l}
0.89 \\
\end{tabular} & 75 \\
\hline & & & $\overline{014}$ & & 71 & 8.5 & 830 & & & 1.24 & & 74. & 15. & & 3.7 & 29.5 & 3.32 & 6.70 & & 1.9 & 0.30 & .056 & \begin{tabular}{|l|}
1.30 \\
\end{tabular} & 36.2 & 33.7 & 1.02 & 590 \\
\hline & & & & & & 7 & & & & & & & & & & & & & & & & & & & 8.6 & 1.1 & 1765 \\
\hline & & & $\overline{005}$ & & & & & & & & & & & & & & & & & & & $\overline{01}$ & $\sqrt{13}$ & 37 & & 10 & 688 \\
\hline & & & .005 & 10 & & 8.2 & 860 & & & 1.45 & & 7.8 & & & 2.8 & 4.2 & 3.24 & 0.30 & 0.19 & 1.7 & 0.40 & .049 & 1.41 & 29.2 & 6.2 & 0.99 & 536 \\
\hline & & & & & & 6.4 & 750 & & & 1.54 & 0. & $\overline{90}$ & & $\overline{1 c}$ & & & 3.27 & & & 2.0 & & & 1.23 & & \begin{tabular}{|l|}
32.1 \\
\end{tabular} & \begin{tabular}{|l|l}
1.04 \\
\end{tabular} & 56 \\
\hline & & & 005 & & & 17.4 & 770 & & & & & & & & 3 & & 4.44 & & & $\overline{1.9}$ & 0.63 & 0 & 1.28 & 32.2 & 3 & 0.96 & 816 \\
\hline & & 54 & .005 & 0.13 & 6.68 & 12.5 & 790 & 1.54 & 0. & 1.49 & 0.34 & $7 \varepsilon$ & & & 2.9 & 30. & 3.81 & 15.80 & 0.22 & 1.9 & 0.45 & 0.056 & 1.20 & 37.8 & \begin{tabular}{|l|l|}
32.4 \\
\end{tabular} & 1.01 & 805 \\
\hline & & & & & & 7.3 & & & & & & & & & & & & & & & $4 \pm$ & & 1.20 & 0.9 & 6. & .98 & 03 \\
\hline & & & 005 & & & 6. & 68 & & & & & & & & 2. & & 3.12 & .20 & & 1 & 0.24 & .050 & & 34.9 & 6 & 0.98 & 514 \\
\hline & & 20 & $<0.005$ & 0.08 & 6.72 & 8.7 & 660 & 1. & 0. & 1.30 & 0.32 & 62.1 & 17.7 & 106 & 3.73 & 21.1 & 3.73 & 15.85 & 0.20 & 1.7 & 0.23 & .059 & 1.12 & 31.0 & 35.6 & 1.04 & 17 \\
\hline & & & & & & & & & & & & & & & & & & & & & 0 & & & 3 & 0.4 & 800 & 53 \\
\hline & 920 & 7 & 0.016 & 0.34 & \begin{tabular}{|l}
6.72 \\
\end{tabular} & .3 & & & 0. & 1.50 & & 62.1 & & 8 & 3. & 2 & 4.88 & 16.50 & 20 & 1.9 & 0.42 & .056 & \begin{tabular}{|l|l|}
1.44 \\
\end{tabular} & 29.4 & \begin{tabular}{|l|}
25.0 \\
\end{tabular} & 1.06 & 4730 \\
\hline & & & 0.010 & 0.26 & \begin{tabular}{|c|c|} 
\\
\end{tabular} & 30.1 & 140 & 1.6 & 0.2 & 1.50 & \begin{tabular}{|l|}
1.11 \\
\end{tabular} & 67 & 40.2 & 8 & 3. & 27. & 5.02 & 16.45 & 0.23 & 1.9 & 0.59 & .052 & 1.44 & 30.1 & 27.2 & 1.04 & 5360 \\
\hline & & & & & & & & & & & & & & & & & 5. & & & 1. & 0.60 & & 1.28 & 33.1 & 4 & 0.98 & 7860 \\
\hline $54 !$ & & 726 & 0.00 & 0.10 & \begin{tabular}{|l|l|}
6.30 \\
\end{tabular} & 9.0 & 810 & 1. & 0.1 & 2.00 & 0.30 & 117 & 15. & 167 & $2.4 \varepsilon$ & & 3.7 & - & & 2.8 & 0.26 & 052 & 1.22 & 58.6 & 8.6 & 1.20 & 945 \\
\hline & & & 0.009 & & & 6.9 & 910 & & & & & 60.1 & 10.4 & 10 & & & 2.72 & & & & 0.14 & & 1.18 & 30.2 & & 0.79 & \\
\hline
\end{tabular}


Table 1. Location and New Trace Element Geochemical Results for 902 Stream-Sediment Samples collected in the Livengood Area, Livengood Quadrangle, Alaska IS = Insufficient Sample for Analysis

\begin{tabular}{|c|c|c|c|c|c|c|c|c|c|c|c|c|c|c|c|c|c|c|c|c|c|c|c|c|c|c|}
\hline SAMPLE & $\begin{array}{l}\text { Mo } \\
\text { ppm }\end{array}$ & $\begin{array}{c}\mathrm{Na} \\
\% \\
\end{array}$ & \begin{tabular}{|l|}
$\mathrm{Nb}$ \\
$\mathrm{ppm}$
\end{tabular} & $\begin{array}{r}\mathrm{Ni} \\
\mathrm{ppm}\end{array}$ & $\begin{array}{c}\mathrm{P} \\
\mathrm{ppm}\end{array}$ & $\begin{array}{c}\mathrm{Pb} \\
\mathrm{ppm}\end{array}$ & $\begin{array}{l}\mathrm{Rb} \\
\mathrm{ppm}\end{array}$ & $\begin{array}{c}\mathrm{Re} \\
\mathrm{ppm}\end{array}$ & $\begin{array}{l}\mathrm{S} \\
\%\end{array}$ & $\begin{array}{l}\mathrm{Sb} \\
\mathrm{ppm}\end{array}$ & \begin{tabular}{|c|} 
Se \\
ppm
\end{tabular} & $\begin{array}{l}S^{S n^{*}} \\
\text { ppm }\end{array}$ & $\begin{array}{c}\mathrm{Sr} \\
\mathrm{ppm}\end{array}$ & $\begin{array}{l}\mathrm{Ta}^{*} \\
\mathrm{ppm}\end{array}$ & $\begin{array}{c}\text { Te } \\
\text { ppm }\end{array}$ & $\begin{array}{c}\text { Th } \\
\text { ppm }\end{array}$ & $\begin{array}{l}\mathrm{Ti}^{*} \\
\%\end{array}$ & $\begin{array}{c}\mathrm{Tl} \\
\mathrm{ppm}\end{array}$ & $\begin{array}{c}\mathrm{U} \\
\mathrm{ppm}\end{array}$ & $\begin{array}{c}\mathrm{V} \\
\mathrm{ppm}\end{array}$ & $\begin{array}{l}W^{*} \\
\text { ppm }\end{array}$ & \begin{tabular}{|c|}
$\mathrm{Y}$ \\
$\mathrm{ppm}$ \\
\end{tabular} & $\begin{array}{l}\mathrm{Zn} \\
\mathrm{ppm}\end{array}$ & $\begin{array}{l}\mathrm{Zr}^{\star} \\
\mathrm{ppm}\end{array}$ & $\begin{array}{c}\text { Pulp Wt } \\
\text { grams }\end{array}$ & $\begin{array}{c}\text { Lab } \\
\text { Report }\end{array}$ \\
\hline 434 & 2.60 & 0.79 & 9.1 & 31.4 & 910 & 14.2 & 64.1 & $<0.002$ & 0.04 & 1.55 & 1 & 1.60 & 136.5 & 0.68 & 0.09 & 7.4 & 0.372 & 0.49 & 3.4 & 138 & 1.0 & 15.1 & 89 & 73.6 & 39 & FA04030730 \\
\hline 464 & 2.58 & 0.65 & 11.9 & 26.9 & 2310 & 22.7 & 79.2 & $<0.002$ & 0.08 & 4.81 & 1 & 2.20 & 202.0 & 0.76 & 0.08 & 12.8 & 0.369 & 1.14 & 5.8 & 218 & 2.5 & 16.6 & 98 & 100.0 & 19 & FA04030730 \\
\hline 465 & 0.74 & 0.54 & 5.9 & 28.8 & 1370 & 9.8 & 34.1 & $<0.002$ & 0.04 & 2.69 & & 1.00 & 149.0 & \begin{tabular}{|l|l|}
0.38 \\
\end{tabular} & $<0.05$ & 5.9 & 0.205 & 0.51 & 2.5 & 82 & 0.9 & 12.9 & 118 & 46.2 & & FA04030730 \\
\hline 468 & .88 & & 10.4 & 33.1 & 810 & 13.8 & 63.5 & $<0,002$ & 0.04 & 3.56 & & 1.60 & 211.0 & 0.75 & $<0.05$ & 8.7 & 0.417 & 0.44 & 2.3 & 116 & 12 & 16.2 & 84 & 69.5 & & \\
\hline 469 & 0.69 & 1.41 & 11.2 & 30.4 & 850 & 12.1 & 60.1 & $<0.002$ & 0.03 & 1.94 & 1 & 1.60 & 226.0 & 0.79 & $<0.05$ & 9.5 & 0.446 & 0.39 & 2.4 & 114 & 1.2 & 16.4 & 80 & 73.7 & 30 & FA04030730 \\
\hline 2 & 1.08 & 1.19 & 10.2 & 28.4 & 680 & 12.8 & 58.2 & $<0.002$ & 0.03 & 0.98 & 1 & 1.80 & 191.0 & 0.74 & $<0.05$ & 8.1 & 0.403 & 0.40 & 2.3 & 114 & 1.0 & 14.7 & 80 & 69.8 & 3 & FA04030730 \\
\hline 473 & 0.94 & 1.28 & \begin{tabular}{|l|}
9.6 \\
\end{tabular} & 37.7 & 720 & 12.3 & 54.9 & $<0.002$ & 0.03 & 0.97 & 1 & 1.60 & 200.0 & 0.70 & \begin{tabular}{|l|}
0.05 \\
\end{tabular} & 8.5 & 0.410 & 0.38 & 2.4 & 116 & 1.0 & 15.0 & 95 & 70.6 & $\overline{14}$ & FA04030730 \\
\hline 474 & 0.83 & 1.38 & 11.0 & 32.8 & 750 & 12.1 & 55.1 & $<0.002$ & 0.02 & 0.98 & & 1.70 & 220.0 & 0.85 & $<0.05$ & 10.3 & 0.460 & 0.35 & 2.4 & 114 & 1.1 & 16.4 & 84 & 77.6 & 30 & FA04030730 \\
\hline & & .45 & $\overline{1.0}$ & 29.0 & 740 & 11.0 & 55.4 & $<0.002$ & 0.02 & 0.93 & & 1.60 & 229.0 & 0.81 & $<0.05$ & 8.8 & 0.443 & 0.38 & 2.4 & 113 & 1.6 & 15.4 & & 70.8 & & FA04030730 \\
\hline 4 & 0.68 & 1.49 & 11.0 & 28.6 & 800 & 11.2 & 54.9 & $<0.002$ & 0.02 & 0.90 & 7 & 1.70 & 238.0 & 0.84 & $<0.05$ & 10.1 & 0.499 & 0.37 & 2.5 & 123 & 1.3 & 16.0 & 97 & 77.6 & 55 & FA04030730 \\
\hline 477 & 0.67 & 1.38 & 9.9 & 27.7 & 730 & 11.3 & 55.2 & $<0.002$ & 0.02 & 1.00 & 1 & 1.50 & 229.0 & 0.70 & $<0.05$ & 8.3 & 0.420 & 0.35 & 2.2 & 113 & 1.2 & 16.0 & 92 & 63.2 & 44 & FA04030730 \\
\hline & 1.98 & 88 & 8.9 & 38.5 & 1040 & 14.6 & 69.4 & $<0.002$ & 0.04 & 1.94 & & 1.90 & 158.0 & 0.65 & \begin{tabular}{|l|}
0.08 \\
\end{tabular} & 8.3 & 0.398 & 0.48 & 3.2 & 174 & 1.1 & 22.4 & & 72.8 & & FA04030730 \\
\hline & 1.04 & 1.07 & 10.2 & 39.0 & 830 & 12.2 & 58.6 & $<0.002$ & 0.04 & 1.28 & 7 & 1.60 & 174.5 & 0.69 & 0.05 & 7.6 & 0.489 & 0.36 & 2.5 & 132 & 1.0 & 17.9 & 94 & 64.3 & 14 & FA04030730 \\
\hline 4 & 0.77 & 1.00 & 9.5 & 38.7 & 850 & 11.8 & 54.6 & $<0.002$ & 0.06 & 1.16 & 1 & 1.70 & 172.0 & 0.66 & $<0.05$ & 7.7 & 0.416 & 0.37 & 2.3 & 116 & 1.0 & 18.2 & 82 & 62.4 & 19 & FA04030730 \\
\hline & 0.79 & 1.20 & 9.9 & 45.5 & 940 & 11.3 & 62.1 & $<0.002$ & 0.04 & 1.16 & & 1.50 & 199.0 & 0.66 & $<0.05$ & 8.6 & 0.410 & 0.37 & 2.3 & 116 & 1.2 & 17.2 & 90 & 62.3 & & FA04030730 \\
\hline & 0.96 & 0.46 & 12.5 & 18.9 & 860 & 24.1 & 64.5 & $<0.002$ & 0.04 & 1.42 & & 3.10 & 168.0 & 0.86 & 0.05 & 11.8 & 0.477 & 0.51 & 2.4 & 132 & 1.5 & 19.3 & 39 & 82.1 & 18 & FA04030730 \\
\hline & 1.34 & 0.74 & 11.2 & 31.8 & 860 & 16.5 & 59.4 & $<0.002$ & 0.05 & 2.42 & & 2.20 & 163.5 & \begin{tabular}{|l|l|}
0.77 \\
\end{tabular} & 0.05 & 9.6 & 0.572 & 0.45 & 2.2 & 178 & 1.2 & 19.0 & 6 & 71.1 & & FA04030730 \\
\hline & 1.14 & 0.84 & 11.6 & 31.7 & 670 & 12.2 & 51.8 & $<0.002$ & 0.04 & 1.64 & 1 & 2.00 & 158.0 & 0.77 & $<0.05$ & 8.2 & 0.717 & 0.39 & 2.0 & 169 & 1. & 16.2 & 7 & & & FA04030730 \\
\hline 5 & 1.16 & 0.82 & 12.2 & 31.1 & 640 & 12.8 & 61.4 & $<0.002$ & 0.05 & 1.32 & 1 & 1.80 & 142.5 & 0.73 & $<0.05$ & 7.5 & 0.531 & 0.40 & 2.1 & 138 & 1.1 & 16.2 & 71 & 71.4 & 22 & FA04030730 \\
\hline & 1.26 & 0.74 & 15.6 & 44.8 & 790 & 12.7 & 66.1 & $<0.002$ & 0.07 & 1.56 & & 2.30 & 137.0 & 0.92 & $<0.05$ & 8.3 & 0.638 & 0.41 & 2.2 & 144 & 1.3 & 20.5 & 134 & 80.2 & & FA04030730 \\
\hline & .48 & .08 & 14.0 & 35.3 & 810 & 13.8 & 70.9 & $<0.002$ & 0.04 & 2.56 & & 1.90 & 185.5 & 0.90 & $<0.05$ & 9.9 & 0.508 & 0.46 & 2.5 & 134 & 1. & 17.8 & 98 & 78. & & \\
\hline & 0.83 & 0.33 & 10.2 & 53.5 & 720 & 13.0 & 52.1 & $<0.002$ & 0.06 & 1.86 & & 2.10 & 99.1 & 0.69 & $<0.05$ & 7.9 & 0.488 & 0.41 & 2.2 & 128 & 1.3 & 22.9 & 150 & 77.6 & 18 & FA04030730 \\
\hline & 0.84 & 0.42 & 8.5 & 52.8 & 1030 & 13.7 & 66.3 & 0.002 & 0.15 & 1.52 & & 2.00 & 126.5 & 0.57 & $<0.05$ & 8.6 & 0.354 & 0.46 & 2.6 & 120 & 1.3 & 21.3 & 176 & 74.1 & & FA04030730 \\
\hline & 2.08 & 0.68 & 17.9 & 43.2 & 1040 & 14.9 & 79.9 & 0.003 & 0.08 & 3.03 & 3 & 2.20 & 147.0 & \begin{tabular}{|l|}
1.02 \\
\end{tabular} & \begin{tabular}{|l|}
0.05 \\
\end{tabular} & 9.6 & 0.476 & 0.48 & 2.3 & 132 & 1.3 & 18.8 & 123 & 89.3 & 4 & FA04030730 \\
\hline & 2.16 & 0.74 & 19.9 & 44.1 & 1320 & 14.2 & 80.3 & 0.003 & 0.07 & 3.87 & & 2.10 & 174.0 & 1.16 & 0.06 & 11.7 & 0.614 & 0.50 & 2.7 & 145 & 1.5 & 20.6 & 124 & 85.7 & & FA04030730 \\
\hline & 2.29 & \begin{tabular}{|l|}
0.82 \\
\end{tabular} & 16.6 & 40.0 & 1140 & 13.7 & 74.5 & 0.004 & 0.06 & 3.64 & 3 & 1.90 & 172.5 & 0.98 & 0.06 & 9.5 & 0.498 & 0.45 & 2.2 & 138 & 2. & \begin{tabular}{|l|}
18.6 \\
\end{tabular} & 116 & 73.3 & & FA04030730 \\
\hline & 1.66 & 0.98 & 11.0 & 33.7 & 840 & 13.8 & 83.3 & 0.002 & 0.06 & 1.36 & & 2.00 & 179.0 & 0.72 & 0.06 & 7.9 & 0.453 & 0.66 & 2.3 & 159 & 1.3 & \begin{tabular}{|l|l|}
18.8 \\
\end{tabular} & 87 & 71.4 & 2 & FA04030730 \\
\hline & 1.12 & 1.15 & 10.5 & 39.8 & 790 & 14.3 & 71.9 & $<0.002$ & 0.04 & 1.08 & & 2.20 & 186.0 & \begin{tabular}{|l|l|}
0.71 \\
\end{tabular} & $<0.05$ & 8.6 & 0.443 & 0.50 & 2.2 & 132 & 1.2 & 17.1 & 90 & 65.0 & 3 & FA04030730 \\
\hline & & 1.04 & 10.0 & 54.3 & 850 & 12.2 & 70.4 & 0.002 & 0.07 & 0.99 & & 1.80 & 189.0 & 0.66 & $<0.05$ & 8.5 & 0.439 & 0.48 & 2.4 & 140 & & 21.1 & 109 & 68. & & \\
\hline & 0.85 & 1.35 & 11.7 & 38.7 & 720 & 12.8 & 72.4 & 0.002 & 0.02 & 1.16 & & 2.10 & 206.0 & 0.83 & $<0.05$ & \begin{tabular}{|l|}
10.1 \\
\end{tabular} & 0.488 & 0.48 & 2.5 & 136 & 1. & \begin{tabular}{|l|}
19.1 \\
\end{tabular} & 96 & 70. & 2 & FA04030730 \\
\hline 5 & 0.79 & 1.35 & 10.2 & 36.0 & 640 & 13.2 & 70.6 & $<0.002$ & 0.03 & 1.30 & 1 & 1.90 & 218.0 & 0.70 & $<0.05$ & 8.3 & 0.407 & 0.41 & 1.9 & 119 & 1.1 & \begin{tabular}{|l|}
16.2 \\
\end{tabular} & 105 & 60.6 & 36 & FA04030730 \\
\hline & 0.67 & 1.41 & 11.8 & 36.5 & 710 & 11.1 & 61.0 & $<0.002$ & 0.04 & 1.02 & & 1.80 & 217.0 & 0.83 & $<0.05$ & 10.5 & 0.512 & 0.37 & 2.3 & 125 & 1.5 & \begin{tabular}{|l|}
18.6 \\
\end{tabular} & 116 & 70.6 & & \\
\hline & 1.51 & 1.32 & \begin{tabular}{|l|}
9.6 \\
\end{tabular} & 41.6 & 1020 & 13.6 & 63.1 & $<0.002$ & 0.04 & 1.34 & & 1.80 & 194.5 & 0.65 & \begin{tabular}{|l|}
0.05 \\
\end{tabular} & \begin{tabular}{|l|}
8.2 \\
\end{tabular} & 0.455 & 0.36 & 2.0 & 150 & 1.0 & \begin{tabular}{|l|}
17.2 \\
\end{tabular} & 104 & 65.0 & 34 & FA04030730 \\
\hline 5 & 1.02 & 1.33 & 10.2 & 39.8 & 800 & 13.6 & 64.9 & $<0.002$ & 0.04 & 1.26 & & 1.90 & 213.0 & 0.70 & $<0.05$ & 9.7 & 0.469 & 0.43 & 2.3 & 142 & 1.7 & \begin{tabular}{|l|l|}
19.4 \\
\end{tabular} & 119 & 72.5 & 26 & FA04030730 \\
\hline & 0.75 & 1.35 & \begin{tabular}{|l|}
10.2 \\
\end{tabular} & 34.2 & 660 & 12.0 & 61.8 & $<0.002$ & 0.04 & 1.04 & & 1.70 & 214.0 & 0.69 & $<0.05$ & 9.0 & 0.440 & 0.38 & 2.2 & 123 & & \begin{tabular}{|l|}
17.4 \\
\end{tabular} & 84 & 60. & & FA04030730 \\
\hline & 0.65 & 1.47 & 10.0 & 32.1 & 660 & 10.3 & 52.9 & $<0.002$ & 0.03 & 0.87 & 1 & 1.70 & 213.0 & 0.69 & $<0.05$ & 9.3 & 0.479 & 0.34 & 2.1 & 122 & 1.2 & \begin{tabular}{|l|l|}
17.8 \\
\end{tabular} & 85 & 61.8 & 5 & FA04030730 \\
\hline 540 & 0.96 & 1.48 & 9.9 & 38.3 & 680 & 13.7 & 57.8 & $<0.002$ & 0.02 & 0.88 & 1 & 1.80 & 167.0 & 0.65 & $<0.05$ & 7.5 & 0.504 & 0.37 & 1.7 & 150 & 0.9 & 17.4 & 102 & 59.1 & 2 & FA04030730 \\
\hline & 1.12 & 1.16 & 11.8 & 34.0 & 640 & 12.0 & 69.2 & & 8 & 0.97 & & 1.90 & 166.5 & & $<0.05$ & 10.2 & 0.519 & 0.44 & 2.6 & 137 & & \begin{tabular}{|l|}
17.6 \\
\end{tabular} & 89 & 78.5 & & FA04030730 \\
\hline 544 & 1.44 & 1.16 & 10.0 & 46.4 & 890 & 21.0 & 74.9 & $<0.002$ & 0.05 & 1.77 & 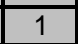 & 2.00 & 206.0 & 0.65 & $<0.05$ & 8.2 & 0.401 & 0.50 & 2.1 & 134 & 1.2 & 20.0 & 165 & 70.3 & 15 & FA04030730 \\
\hline & 1.59 & 1.15 & 9.9 & 50.7 & 930 & 17.9 & 76.6 & $<0.002$ & 0.05 & 1.78 & & 1.90 & 209.0 & 0.65 & \begin{tabular}{|l|}
0.05 \\
\end{tabular} & 8.5 & 0.377 & 0.50 & 2.1 & 136 & 1.2 & \begin{tabular}{|l|l|}
18.8 \\
\end{tabular} & 128 & 69.0 & 23 & 730 \\
\hline & 1.80 & 1.07 & 9.0 & 55.2 & 1060 & 19.5 & 69.4 & $<0.002$ & 0.06 & 1.83 & 2 & 1.70 & 202.0 & 0.61 & $<0.05$ & 8.7 & 0.368 & 0.46 & 2.0 & 138 & 1.0 & \begin{tabular}{|l|}
20.2 \\
\end{tabular} & 150 & 67.6 & 24 & FA0403C \\
\hline 547 & 0.94 & 1.36 & 14.4 & 38.5 & 910 & 12.2 & 59.2 & $<0.002$ & 0.02 & 1.24 & 2 & 1.90 & 235.0 & 1.06 & $<0.05$ & 14.2 & 0.622 & 0.37 & 3.1 & 134 & 1.4 & 22.9 & 85 & 96.2 & 35 & 0730 \\
\hline 549 & 1.24 & 1.23 & 10.3 & 26.5 & 700 & 11.8 & 61.6 & 0.002 & 0.04 & 1.06 & 2 & 1.70 & 203.0 & 0.72 & $<0.05$ & 8.0 & 0.406 & 0.42 & 2.4 & 118 & 1.2 & 15.4 & 90 & 73.2 & 15 & FA04030730 \\
\hline
\end{tabular}


Table 1. Location and New Trace Element Geochemical Results for 902 Stream-Sediment Samples collected in the Livengood Area, Livengood Quadrangle, Alaska IS = Insufficient Sample for Analysis

\begin{tabular}{|c|c|c|c|c|c|c|c|c|c|c|c|c|c|c|c|c|c|c|c|c|c|c|c|c|c|c|c|}
\hline MPLE & TM E & TM N & $\begin{array}{c}\mathrm{Au} \\
\mathrm{ppm}\end{array}$ & $\begin{array}{c}\mathrm{Ag} \\
\mathrm{ppm}\end{array}$ & $\begin{array}{l}\text { Al } \\
\%\end{array}$ & $\begin{array}{l}\text { As } \\
\text { ppm }\end{array}$ & $\begin{array}{l}\mathrm{Ba}^{*} \\
\mathrm{ppm}\end{array}$ & \begin{tabular}{|c|}
$\mathrm{Be}$ \\
$\mathrm{ppm}$ \\
\end{tabular} & $\begin{array}{c}\mathrm{Bi} \\
\mathrm{ppm}\end{array}$ & $\begin{array}{c}\mathrm{Ca} \\
\% \\
\end{array}$ & \begin{tabular}{|c|}
$\mathrm{Cd}$ \\
$\mathrm{ppm}$ \\
\end{tabular} & $\begin{array}{c}\mathrm{Ce} \\
\mathrm{ppm}\end{array}$ & $\begin{array}{l}\mathrm{Co} \\
\mathrm{ppm}\end{array}$ & $\begin{array}{l}\mathrm{Cr}^{*} \\
\mathrm{ppm}\end{array}$ & $\begin{array}{l}\text { CS } \\
\text { ppm }\end{array}$ & $\begin{array}{r}\mathrm{Cu} \\
\mathrm{ppm}\end{array}$ & $\begin{array}{c}\mathrm{Fe} \\
\% \\
\end{array}$ & $\begin{array}{c}\mathrm{Ga} \\
\mathrm{ppm}\end{array}$ & $\begin{array}{c}\mathrm{Ge} \\
\mathrm{ppm}\end{array}$ & $\begin{array}{c}\mathrm{Hf} \\
\mathrm{ppm} \\
\end{array}$ & $\begin{array}{l}\mathrm{Hg} \\
\mathrm{ppm}\end{array}$ & $\begin{array}{c}\text { In } \\
\mathrm{ppm}\end{array}$ & $\begin{array}{l}\mathrm{K} \\
\% \\
\end{array}$ & $\begin{array}{r}\mathrm{La} \\
\mathrm{ppm}\end{array}$ & $\begin{array}{c}\mathrm{Li} \\
\mathrm{ppm} \\
\end{array}$ & $\begin{array}{c}\mathrm{Mg} \\
\% \\
\end{array}$ & $\begin{array}{l}\mathrm{Mn} \\
\mathrm{ppm}\end{array}$ \\
\hline 550 & 6051 & 81994 & 0.007 & $\begin{array}{l}0.12 \\
\end{array}$ & \begin{tabular}{|l|l}
6.10 \\
\end{tabular} & 6.7 & 950 & 1.50 & 0.13 & 1.68 & \begin{tabular}{|l}
0.32 \\
\end{tabular} & 69.7 & 110 & 83 & 2.53 & 16.8 & 2.87 & 14.55 & 0.20 & 2.2 & 0.08 & 0.045 & 1.25 & 34.1 & 23.8 & 0.92 & 492 \\
\hline 51 & & & 005 & & \begin{tabular}{|l}
6.17 \\
\end{tabular} & 7.0 & 950 & 1.66 & & 96 & \begin{tabular}{|l|}
0.30 \\
\end{tabular} & 61.3 & 11. & & 2.33 & 8. & 2.99 & 14.45 & 0.19 & 2.0 & 0.10 & 0.045 & \begin{tabular}{|l}
1.31 \\
\end{tabular} & 29.7 & 1.8 & 1.03 & 528 \\
\hline & & & 0.005 & 10 & 5.84 & 7.6 & 920 & 1.40 & 0.12 & 1.83 & 33 & 3.2 & $\begin{array}{l}11.3 \\
\end{array}$ & 80 & 2.19 & 8.2 & 3.06 & 3.65 & 0.29 & 2.2 & 0.10 & 0.038 & \begin{tabular}{|l|}
1.22 \\
\end{tabular} & 29.7 & 0.5 & 0.99 & 573 \\
\hline & & & 0.012 & & 6.15 & 11.1 & 90 & & & & & & & & & & & 4.95 & 028 & 21 & & & 1.38 & 27.7 & 34.5 & 0.96 & 1490 \\
\hline & & 32738 & .010 & .07 & \begin{tabular}{|l}
5.89 \\
\end{tabular} & 6.6 & 910 & .36 & 0.12 & 1.64 & 0.34 & 53.9 & 11.6 & 72 & 2.30 & 7.2 & 2.86 & 13.75 & 0.28 & 2.0 & 0.11 & 0.034 & 1.26 & 26.4 & 31.9 & 0.91 & 745 \\
\hline & & & 011 & 07 & 6.01 & $\overline{7.6}$ & 930 & 1.50 & 0.12 & 1.70 & 0.28 & 59.3 & 11.6 & 79 & 2.39 & 18.9 & 3.02 & 14.40 & 0.29 & 2.2 & 0.10 & .038 & 1.26 & 29.2 & 30.6 & 0.96 & 552 \\
\hline & & & $\overline{005}$ & & 5.65 & $\overline{7.1}$ & 890 & 1.29 & & 1.56 & \begin{tabular}{|l|}
0.31 \\
\end{tabular} & 52.8 & & & 2.42 & & 285 & & 0.31 & 1.9 & & & & 26.0 & 32.7 & 0.90 & \\
\hline & & & 0.005 & 0.09 & 5.84 & 7.5 & 910 & 1.44 & 0.13 & 1.62 & \begin{tabular}{|l|}
0.35 \\
\end{tabular} & 55.7 & 12.4 & 7 & 2.39 & 19.6 & 3.02 & 13.85 & 0.27 & 2.0 & 0.09 & 0.038 & 1.23 & 27.3 & 29.1 & 0.92 & $\overline{794}$ \\
\hline & & & 005 & 07 & 5.65 & & 890 & 1.36 & 0.11 & 1.68 & 0.29 & 2.3 & 11.1 & 13 & 2.21 & 7.8 & 2.85 & 13.30 & 0.25 & 2.0 & 0.15 & 0.036 & 1.23 & 25.6 & 27.3 & 0.93 & 578 \\
\hline & & & 008 & 06 & \begin{tabular}{|l}
5.73 \\
\end{tabular} & . & 840 & 1.31 & 0.10 & 2.03 & \begin{tabular}{|l|l}
0.27 \\
\end{tabular} & 6.8 & 11.1 & 112 & 2.00 & 2.2 & 3.13 & 13.65 & 0.32 & 3.2 & 0.06 & 0.041 & \begin{tabular}{ll|}
1.15 \\
\end{tabular} & 46.8 & 25.1 & 1.02 & 639 \\
\hline & & & 0.005 & 0.07 & 5.70 & 6.5 & 870 & 1.43 & 0.11 & 1.84 & 0.21 & 64.6 & 10.5 & & 2.03 & 15.6 & 2.87 & 13.45 & 0.29 & 2.2 & 0.10 & 0.037 & \begin{tabular}{|l|}
1.19 \\
\end{tabular} & 30.0 & 27.1 & 0.95 & 484 \\
\hline & & & 005 & 05 & \begin{tabular}{|l|l}
5.69 \\
\end{tabular} & & 850 & .24 & 0.10 & 1.90 & \begin{tabular}{|l|}
0.20 \\
\end{tabular} & 9. & 10.6 & 10 & 1.95 & . & 3.02 & 13.55 & 0.31 & 2.9 & 0.05 & 0.041 & \begin{tabular}{|l|l|}
1.15 \\
\end{tabular} & 42.1 & 23.6 & 0.95 & 585 \\
\hline & & & .005 & $\overline{09}$ & .74 & & 930 & $\overline{31}$ & 10 & 1.86 & 0.30 & $\overline{9.0}$ & 11. & & 205 & 8 & 3.14 & 13.45 & 0.34 & 23 & 1.04 & .041 & \begin{tabular}{|l|l|}
1.14 \\
\end{tabular} & 31 & 4.7 & 0.98 & 25 \\
\hline & & & .005 & 0.22 & 5.86 & 6.8 & 770 & 1.26 & 0.15 & 1.46 & 0.33 & 54.6 & 14.3 & 8 & 2.66 & 40.9 & 3.46 & 14.60 & 0.28 & 2.2 & 0.13 & 0.044 & 1.00 & 26.3 & 24.9 & 0.94 & 768 \\
\hline & & & 006 & & \begin{tabular}{|l|l}
6.08 \\
\end{tabular} & & 950 & & & 1.84 & 035 & & 12.3 & & 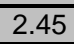 & 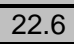 & 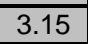 & 4.85 & 0.30 & 2.2 & 0.19 & 041 & \begin{tabular}{|l|}
1.28 \\
\end{tabular} & 30.7 & 29.2 & \begin{tabular}{|l|}
1.02 \\
\end{tabular} & 552 \\
\hline & & & .005 & 0.08 & 5.89 & 6.8 & 910 & 1.42 & 0.12 & 1.80 & 0.29 & 63.7 & 12 . & 8 & 2.17 & 21.8 & 3.01 & 14.05 & 0.28 & 2.2 & 0.09 & .042 & 1.21 & 29.4 & 6.5 & 0.99 & 540 \\
\hline & & & .005 & .09 & 5.98 & 6. & 890 & 1.30 & 0.12 & 1.87 & 0.26 & 99.9 & 11. & & 2.15 & $19.2 \mathrm{Z}$ & 3.06 & 14.20 & 0.28 & 2.5 & 0.18 & .041 & 1.21 & 32.5 & 25.2 & 1.04 & 496 \\
\hline & & & 005 & 07 & 5.82 & & 880 & & & 87 & & & & & & & 3.06 & 3.45 & 0.30 & 2. & 0.06 & $\overline{038}$ & 1.16 & 31.3 & 4.2 & 1.06 & $\overline{24}$ \\
\hline & & & .005 & 0.07 & 5.64 & 6.8 & 870 & 1.36 & 0.11 & 1.85 & \begin{tabular}{|l}
0.26 \\
\end{tabular} & 65.9 & 11.3 & 7 & 2.09 & 17.3 & 2.91 & 13.70 & 0.34 & 2.1 & 0.15 & $\overline{039}$ & 1.18 & 30.5 & 24.9 & 1.00 & 490 \\
\hline & & & .005 & & 5.82 & & 920 & & & 1.88 & 0.4 & 6. & 12. & & 2.4 & 24. & 3.16 & 13.85 & 0.32 & 2.3 & 0.20 & .042 & $1.2 \mathrm{Y}$ & 30.4 & 24.8 & \begin{tabular}{|l|}
1.08 \\
\end{tabular} & 619 \\
\hline & & & .005 & 08 & \begin{tabular}{|l|l|}
6.13 \\
\end{tabular} & 8.0 & 960 & & & 2.15 & & 80.3 & 11. & & 2.2 & 18. & 3.18 & 4.40 & 0.33 & & 0.08 & .044 & 1.32 & 37.5 & 26.9 & 1.16 & 530 \\
\hline & & & 0.006 & 0.09 & 5.80 & 7. & 930 & 1.36 & 0.12 & 1.66 & \begin{tabular}{|l}
0.32 \\
\end{tabular} & 63.0 & 9.4 & & 2.34 & 23.4 & 2.88 & 13.85 & 0.29 & 2.1 & 0.21 & .042 & 1.26 & 29.6 & 26.5 & 0.96 & 356 \\
\hline & & & 016 & 0.24 & 6.40 & 7. & 020 & 1.4 & & 1.64 & 0.5 & 59. & 13. & & $3.1 \mathrm{Y}$ & 02. & 3.62 & 17.60 & 0.35 & 2.6 & 0.92 & .066 & 0.91 & 30.2 & 27.6 & 0.91 & 306 \\
\hline & & & 005 & 10 & 90 & 6 & 200 & & & 60 & & 46. & 18. & & & & & 16. & & 2.6 & & & 0.92 & 21.6 & \begin{tabular}{|l|l}
21.2 \\
\end{tabular} & 1.03 & 24 \\
\hline & & & .005 & .09 & 6.96 & 4. & 1020 & .08 & 0.09 & 2.20 & 0.61 & 40. & 21.2 & & 1.9 & 40.7 & 5.03 & 17.35 & 0.36 & 2. & 0.52 & 0.058 & 0.79 & 19.1 & 22.9 & 1.46 & 783 \\
\hline & & & .005 & 15 & & 5.3 & 970 & & & 1.90 & & & & & . & .7 & 4.92 & 6.45 & 0.34 & 2. & .90 & .052 & 0.93 & 24.1 & 26.0 & 1.21 & 825 \\
\hline & & & .005 & 18 & \begin{tabular}{|l|l|} 
\\
\end{tabular} & 12.2 & 900 & 1.29 & 0.1 & 1.66 & \begin{tabular}{|l}
1.47 \\
\end{tabular} & 52.1 & 16.7 & & 2.45 & 24.2 & 3.71 & 12.00 & 0.31 & 1.7 & 0.50 & 038 & \begin{tabular}{|l|}
1.06 \\
\end{tabular} & 24.6 & 5.4 & 0.83 & 1955 \\
\hline & & & .005 & 08 & .73 & 6.3 & 890 & 1.4 & & 1.78 & & 66. & 10. & & 2.1 & 16. & 2.87 & 13.60 & 0.29 & 2.3 & 0.19 & .038 & \begin{tabular}{|l|}
1.24 \\
\end{tabular} & 31.0 & 27.0 & 0.98 & 472 \\
\hline & & & 005 & .17 & 5.60 & 6.5 & 300 & & & 1.19 & & & & & & & & & & & 0.5 & & 1.2 & 27. & 37.2 & 0.9 & 6290 \\
\hline & & & 005 & & & 7 & 910 & & & & & & & & & & 30 & 465 & & 2 & $\overline{01}$ & $\overline{0}$ & & & 30 & $\overline{0 .}$ & 518 \\
\hline & & & .005 & 0.11 & 6.26 & 8.0 & 950 & 1.51 & & \begin{tabular}{ll|}
1.66 \\
\end{tabular} & .31 & 54.9 & & & 2.6 & 0.7 & 3.09 & 14.85 & 0.29 & 2.0 & 0.33 & .041 & 1.36 & 26.5 & 28.0 & 0.98 & 487 \\
\hline & & & .005 & & 5.91 & 6.8 & 870 & & & \begin{tabular}{|l|}
1.70 \\
\end{tabular} & \begin{tabular}{|l|l}
0.33 \\
\end{tabular} & 62 & & & & & 2.98 & 4.30 & 0.29 & 2.1 & 11 & & 1.25 & 29.2 & 26.1 & 0.95 & 669 \\
\hline & & & 005 & & .96 & 7. & 870 & & & \begin{tabular}{|l|}
1.78 \\
\end{tabular} & & & & & 2. & & 3.06 & 4.20 & 29 & 2.4 & 0.13 & 0 & 1.23 & 35.4 & 24.0 & 0.96 & 722 \\
\hline & 372 & 19 & $<0.005$ & 0.09 & 6.19 & 8.7 & 950 & 1.34 & 0.1 & 1.76 & \begin{tabular}{|l}
0.39 \\
\end{tabular} & 63.9 & 13.2 & & 2.61 & 2.6 & 3.23 & 14.65 & 0.30 & 2.1 & 0.18 & .041 & 1.32 & 30.1 & 26.7 & 0.98 & 858 \\
\hline & & & & & & 7.8 & 910 & & & \begin{tabular}{|l|}
1.86 \\
\end{tabular} & & & & & & & 3.12 & & & 2.8 & & & 1.24 & 40.9 & 3.9 & 1.00 & 40 \\
\hline & & & .005 & & 5. & 9. & 950 & & & 1.82 & & 66 & & & 2. & & 3.27 & 4.00 & & 2.3 & 0.12 & $\overline{039}$ & 1.22 & 31.3 & 26.4 & 0.99 & 601 \\
\hline & & 2.14 & 0.013 & 0.06 & 6.06 & 7.2 & 960 & 1.37 & 0. & 1.94 & \begin{tabular}{|l}
0.28 \\
\end{tabular} & 88.8 & 11.8 & 104 & 2.25 & 0.8 & 3.32 & 14.35 & 0.34 & 2.7 & 0.09 & .045 & \begin{tabular}{|l|l|}
1.24 \\
\end{tabular} & 41.3 & \begin{tabular}{|l|}
23.8 \\
\end{tabular} & 1.04 & 774 \\
\hline & & & & & & & & & & 2.13 & & & & & & & & & & & 80 & {[} & 1.32 & 318 & 8.2 & 110 & 59 \\
\hline & & & $<0.005$ & 0.08 & \begin{tabular}{|l|l|}
6.15 \\
\end{tabular} & 7.2 & 940 & & 0. & 1.83 & \begin{tabular}{|l}
0.28 \\
\end{tabular} & 57.9 & 12 & & 2.4 & & 3.05 & 14.90 & 29 & 2.0 & 0.14 & .038 & 1.31 & 27.3 & 6.1 & 1.02 & 511 \\
\hline & & & 0.007 & 0.07 & 5.98 & 7.0 & 900 & 1. & 0. & 1.88 & \begin{tabular}{|l|l|}
0.26 \\
\end{tabular} & 64.7 & 12.4 & 8 & 2.20 & & 3.08 & 14.70 & 0.30 & 2.2 & 0.08 & 1.039 & \begin{tabular}{|l|}
1.22 \\
\end{tabular} & 30.2 & 24.7 & 1.02 & 523 \\
\hline & & & & & & 5. & 860 & & & & & 73.7 & & & 2. & & 3.02 & & & 2.5 & 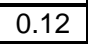 & & 1.17 & 34.0 & 22. & 1.03 & 522 \\
\hline 602 & & 7284452 & 0.015 & 0.04 & 5.80 & 5.7 & 890 & 1.22 & 0.1 & 2.01 & \begin{tabular}{|l|l|} 
\\
\end{tabular} & 95.4 & 11.6 & 106 & 1.9 & 15.2 & 3.19 & 13.90 & 0 & 3.0 & 0.08 & 0 & 1.16 & 44.4 & 11.6 & 1.04 & 597 \\
\hline & & 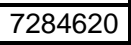 & $<0.005$ & 0.11 & & 6.5 & 890 & & 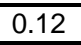 & & & 59.1 & 11.7 & & 2.10 & 19.4 & 2.95 & & & \begin{tabular}{|l|l} 
\\
\end{tabular} & 0.14 & & \begin{tabular}{|l|l|} 
\\
\end{tabular} & 27.8 & & 0.97 & \\
\hline
\end{tabular}


Table 1. Location and New Trace Element Geochemical Results for 902 Stream-Sediment Samples collected in the Livengood Area, Livengood Quadrangle, Alaska IS = Insufficient Sample for Analysis

\begin{tabular}{|c|c|c|c|c|c|c|c|c|c|c|c|c|c|c|c|c|c|c|c|c|c|c|c|c|c|c|}
\hline SAMPLE & $\begin{array}{l}\text { Mo } \\
\text { ppm }\end{array}$ & $\begin{array}{c}\mathrm{Na} \\
\%\end{array}$ & $\begin{array}{l}\mathrm{Nb} \\
\mathrm{ppm}\end{array}$ & $\begin{array}{r}\mathrm{Ni} \\
\mathrm{ppm}\end{array}$ & $\begin{array}{c}\mathrm{P} \\
\mathrm{ppm}\end{array}$ & $\begin{array}{l}\mathrm{Pb} \\
\mathrm{ppm}\end{array}$ & $\begin{array}{l}\mathrm{Rb} \\
\mathrm{ppm}\end{array}$ & $\begin{array}{l}\mathrm{Re} \\
\mathrm{ppm}\end{array}$ & $\begin{array}{l}\mathrm{S} \\
\%\end{array}$ & $\begin{array}{c}\mathrm{Sb} \\
\mathrm{ppm}\end{array}$ & \begin{tabular}{c|} 
Se \\
ppm
\end{tabular} & $\begin{array}{l}S^{S} n^{*} \\
\text { ppm }\end{array}$ & $\begin{array}{c}\mathrm{Sr} \\
\mathrm{ppm}\end{array}$ & $\begin{array}{l}\mathrm{Ta}^{*} \\
\mathrm{ppm}\end{array}$ & $\begin{array}{c}\text { Te } \\
\text { ppm }\end{array}$ & $\begin{array}{c}\text { Th } \\
\text { ppm }\end{array}$ & $\begin{array}{l}\mathrm{Ti}^{\star} \\
\% \\
\end{array}$ & \begin{tabular}{c|}
$\mathrm{Tl}$ \\
$\mathrm{ppm}$
\end{tabular} & $\begin{array}{c}\mathrm{U} \\
\mathrm{ppm}\end{array}$ & $\begin{array}{c}\mathrm{V} \\
\mathrm{ppm}\end{array}$ & $\begin{array}{l}W^{*} \\
\text { ppm }\end{array}$ & \begin{tabular}{|c|}
$\mathrm{Y}$ \\
$\mathrm{ppm}$
\end{tabular} & $\begin{array}{l}\text { Zn } \\
\text { ppm }\end{array}$ & $\begin{array}{l}\mathrm{Zr}^{*} \\
\mathrm{ppm}\end{array}$ & $\begin{array}{c}\text { Pulp Wt } \\
\text { grams }\end{array}$ & $\begin{array}{c}\text { Lab } \\
\text { Report }\end{array}$ \\
\hline 550 & 0.87 & 1.42 & 11.2 & 29.8 & 790 & 11.4 & 61.4 & $<0.002$ & 0.02 & 1.12 & \begin{tabular}{|l|}
2 \\
\end{tabular} & 1.60 & 236.0 & 0.76 & $<0.05$ & 8.8 & 0.435 & 0.40 & 2.5 & 119 & 1.0 & 17.8 & 144 & 78.0 & 45 & FA04030730 \\
\hline 551 & 0.89 & 1.48 & 10.5 & 30.4 & 870 & 11.1 & 58.8 & $<0.002$ & 0.02 & 1.08 & 1 & 1.50 & 253.0 & 0.72 & $<0.05$ & 8.0 & 0.413 & 0.38 & 2.1 & 118 & 1.2 & 16.9 & 91 & 67.3 & 50 & FA04030730 \\
\hline 552 & 0.85 & 1.34 & 10.2 & 28.6 & 880 & 11.6 & 55.0 & $<0.002$ & 0.03 & 1.20 & 1 & 1.50 & 231.0 & \begin{tabular}{|l|}
0.72 \\
\end{tabular} & $<0.05$ & 8.6 & 0.424 & 0.33 & 2.2 & 116 & 1.2 & 16.6 & 151 & 67.6 & 41 & FA04030730 \\
\hline 553 & 1.08 & 24 & 10.4 & 34.0 & 870 & 12.4 & 66.0 & $<0.002$ & 0.03 & 1.12 & & 1.60 & 214.0 & \begin{tabular}{|l|}
0.69 \\
\end{tabular} & \begin{tabular}{|l|}
0.05 \\
\end{tabular} & 8.4 & 0.402 & 0.43 & 2.2 & 126 & 1.2 & 16.6 & 124 & 66.4 & & \\
\hline 554 & 0.77 & 1.33 & 9.4 & 27.5 & 810 & 11.5 & 58.2 & 0.002 & 0.03 & 1.12 & 2 & 1.50 & 226.0 & \begin{tabular}{|l|l|}
0.66 \\
\end{tabular} & $<0.05$ & 8.0 & 0.381 & 0.35 & 2.0 & 110 & 1.1 & 15.2 & 118 & 62.5 & 19 & FA04030730 \\
\hline 55 & 0.86 & 1.35 & 10.4 & 29.1 & 820 & 11.4 & 58.7 & $<0.002$ & 0.03 & 0.98 & 2 & 1.50 & 230.0 & \begin{tabular}{|l|}
0.74 \\
\end{tabular} & $<0.05$ & 9.0 & 0.419 & 0.36 & 2.2 & 118 & 1.0 & 16.4 & 87 & 71.6 & 36 & FA04030730 \\
\hline 556 & .90 & 1.24 & 9.4 & 29.5 & 760 & 11.5 & 59.1 & 0.002 & 0.03 & 2.22 & 1 & 1.50 & 211.0 & \begin{tabular}{|l|}
0.67 \\
\end{tabular} & 0.05 & 8.0 & 0.371 & 0.35 & 2.0 & 110 & 0.9 & 15.2 & 92 & 62.0 & & \\
\hline 558 & 0.88 & 1.27 & 9.7 & 28.9 & 730 & 11.1 & 59.1 & $\begin{array}{l}<0.002 \\
\end{array}$ & 0.04 & 1.14 & 1 & 1.50 & 216.0 & \begin{tabular}{|l|l|}
0.68 \\
\end{tabular} & $<0.05$ & 7.7 & 0.390 & 0.37 & 2.1 & 113 & 1.0 & 15.4 & 110 & 62.9 & 29 & FA04030730 \\
\hline 5 & 0.81 & 1.30 & \begin{tabular}{|l|}
9.5 \\
\end{tabular} & 27.6 & 800 & 10.8 & 54.9 & $<0.002$ & 0.03 & 1.16 & & 1.40 & 222.0 & \begin{tabular}{|l|l|}
0.68 \\
\end{tabular} & $<0.05$ & \begin{tabular}{|l|}
7.8 \\
\end{tabular} & 0.384 & 0.34 & 2.0 & 110 & 1.0 & 15.1 & 142 & 62.0 & 30 & FA04030730 \\
\hline 560 & 67 & 1.34 & 13.5 & 26.3 & 950 & 10.0 & 51.6 & $<0.002$ & 0.02 & 0.88 & & 1.50 & 242.0 & \begin{tabular}{|l|l|}
1.08 \\
\end{tabular} & $<0.05$ & \begin{tabular}{|l|l}
12.7 \\
\end{tabular} & 0.574 & 0.29 & 3.0 & 124 & 1.6 & 20.5 & 90 & 96.6 & & FA04030730 \\
\hline 561 & 0.74 & 1.35 & 10.2 & 26.8 & 800 & 10.7 & 52.4 & \begin{tabular}{l|}
$<0.002$ \\
\end{tabular} & 0.02 & 1.00 & & 1.40 & 232.0 & 0.75 & $<0.05$ & 9.0 & 0.435 & 0.32 & 2.2 & 116 & 1.1 & 16.4 & 94 & 64.6 & 4 & FA04030730 \\
\hline 5 & 0.80 & 1.35 & 12.1 & 26.2 & 870 & 10.4 & 50.6 & \begin{tabular}{|l|}
0.002 \\
\end{tabular} & 0.02 & 0.86 & & 1.50 & 238.0 & \begin{tabular}{|l|l|}
0.88 \\
\end{tabular} & $<0.05$ & 11.9 & 0.542 & 0.29 & 2.7 & 120 & 1.2 & 19.1 & 83 & 90.0 & 4 & FA04030730 \\
\hline 5 & 0.87 & 1.33 & 10.9 & 27.7 & 840 & 10.6 & 51.7 & 0.002 & 0.02 & 0.93 & & 1.50 & 227.0 & \begin{tabular}{|l|}
0.79 \\
\end{tabular} & $<0.05$ & 9.0 & 0.505 & 0.33 & 2.5 & 124 & 1.6 & 17.3 & 111 & 72.9 & & FA04030730 \\
\hline 565 & 1.84 & 1.03 & 10.0 & 30.3 & 690 & 11.7 & 58.4 & 0.002 & 0.04 & 1.00 & & 1.60 & 179.5 & \begin{tabular}{|l|l|}
0.71 \\
\end{tabular} & 0.05 & 7.4 & 0.447 & 0.42 & 2.4 & 140 & 1.0 & 16.2 & 97 & 76.8 & 2 & FA04030730 \\
\hline & 0.92 & 1.35 & 10.8 & 31.7 & 820 & 12.0 & 60.1 & $<0.002$ & 0.03 & 1.08 & & 1.50 & 235.0 & 0.73 & $<0.05$ & 8.9 & 0.446 & 0.36 & 2.2 & 124 & 1.0 & 17.5 & 91 & 74.3 & & FA04030730 \\
\hline 68 & 0.91 & 1.36 & 10.4 & 28.8 & 770 & 11.7 & 54.7 & $<0.002$ & 0.03 & 0.99 & 2 & 1.40 & 231.0 & \begin{tabular}{|l|}
0.72 \\
\end{tabular} & $<0.05$ & 8.9 & 0.430 & 0.34 & 2.2 & 120 & 1.2 & 16.9 & 86 & 70.3 & & FA04030730 \\
\hline 569 & 0.89 & 1.42 & 11.4 & 28.6 & 820 & 11.4 & 52.8 & $<0.002$ & 0.02 & 1.06 & & 1.50 & 242.0 & \begin{tabular}{|l|}
0.81 \\
\end{tabular} & $<0.05$ & 9.3 & 0.502 & 0.33 & 2.4 & 126 & 1.2 & 17.8 & 9 & 74.4 & o & FA04030730 \\
\hline & $\overline{82}$ & 1.42 & 10.4 & 26.8 & 750 & 0.8 & 50.3 & $<0.002$ & 0.02 & 0.90 & 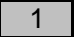 & 1.40 & 238.0 & \begin{tabular}{|l|l|}
0.74 \\
\end{tabular} & $<0.05$ & 9.2 & 0.459 & 0.32 & 2.4 & 121 & 1.1 & 16.4 & & 68.1 & & \\
\hline 571 & 0.83 & 1.39 & 10.6 & 28.2 & 770 & 11.3 & 53.2 & $<0.002$ & 0.03 & 0.96 & & 1.40 & 237.0 & 0.75 & $<0.05$ & 8.8 & 0.436 & 0.32 & 2.2 & 115 & 1.0 & 16.8 & 87 & 67.7 & 82 & 30730 \\
\hline 5 & 1.25 & 1.31 & 10.5 & 31.8 & 820 & 12.0 & 54.9 & \begin{tabular}{|l|}
0.003 \\
\end{tabular} & 0.04 & 1.13 & & 1.50 & 230.0 & 0.73 & $<0.05$ & 9.0 & 0.441 & 0.37 & 2.4 & 126 & 1.0 & 18.0 & 103 & 77.1 & & A04030730 \\
\hline & 0.96 & 1.46 & 12.0 & 30.3 & 980 & 11.6 & 56.3 & $<0.002$ & 0.03 & 1.11 & 2 & 1.50 & 260.0 & 0.86 & $<0.05$ & 11.8 & 0.482 & 0.34 & 2.5 & 124 & 1.2 & 19.3 & 98 & 80.1 & & A04030730 \\
\hline 576 & 0.89 & 1.30 & \begin{tabular}{|l|}
9.9 \\
\end{tabular} & 28.5 & 810 & 11.6 & 56.2 & $<0.002$ & 0.04 & 1.12 & & 1.40 & 224.0 & \begin{tabular}{|l|l|} 
& \\
\end{tabular} & $<0.05$ & 8.8 & 0.400 & 0.36 & 2.2 & 117 & 0.9 & 17.4 & 93 & 64.3 & 7 & FA04030730 \\
\hline 5 & 1.91 & 1.23 & 10.0 & 35.6 & 940 & 12.1 & 52.5 & 0.003 & 0.06 & 0.79 & & 1.90 & 167.0 & \begin{tabular}{|l|}
0.67 \\
\end{tabular} & $<0.05$ & 6.7 & 0.503 & 0.42 & 2.7 & 152 & 0.9 & 28.1 & 117 & 87.5 & & FA04030730 \\
\hline & 1.48 & 1.76 & 10.7 & 29.3 & 800 & 10.9 & 44.1 & 0.002 & 0.03 & 0.74 & & 1.60 & 200.0 & 0.72 & $<0.05$ & 6.0 & 0.651 & 0.32 & 2.1 & 180 & 1. & 18.6 & 116 & 79 & & 7730 \\
\hline 5 & 1.20 & 1.76 & 9.9 & 46.0 & 760 & 8.8 & 38.8 & 0.002 & 0.03 & 0.64 & & 1.50 & 217.0 & \begin{tabular}{|l|}
0.65 \\
\end{tabular} & $<0.05$ & 4.7 & 0.615 & 0.24 & 1.5 & 182 & $\overline{0.9}$ & 19.7 & 143 & 78.1 & & FA04030730 \\
\hline & 1.31 & 1.32 & 8.9 & 44.7 & 820 & 9.6 & 49.1 & 0.003 & 0.06 & 0.89 & & 1.50 & 197.0 & \begin{tabular}{|l|}
0.61 \\
\end{tabular} & $<0.05$ & 5.7 & 0.456 & 0.35 & 1.8 & 156 & 0. & 21.6 & 206 & 77. & & FA04030730 \\
\hline & 1.22 & 0.98 & 8.0 & 33.0 & 1350 & 12.0 & 58.8 & 0.002 & 0.11 & 1.30 & 2 & 1.50 & 185.0 & \begin{tabular}{|l|}
0.61 \\
\end{tabular} & $<0.05$ & 7.5 & 0.311 & 0.36 & 1.9 & 103 & 0.9 & 15.3 & 118 & 52.9 & & 30730 \\
\hline 5 & 0.78 & 1.40 & 11.0 & 28.3 & 840 & 10.9 & 53.6 & $<0.002$ & 0.02 & 1.00 & & 1.40 & 235.0 & \begin{tabular}{|l|}
0.81 \\
\end{tabular} & $<0.05$ & 9.1 & 0.437 & 0.33 & 2.4 & 111 & 1.2 & 16.6 & 101 & 70.5 & 5 & A04030730 \\
\hline & 1.60 & 1.05 & 8.8 & 44.9 & 1040 & 14.0 & 65.0 & \begin{tabular}{|l|}
0.002 \\
\end{tabular} & 0.0 & 1.28 & & 1. & 201.0 & \begin{tabular}{|l|}
0.62 \\
\end{tabular} & $<0.05$ & 7.8 & 0.336 & 0.41 & 1.9 & 12 & & 16.8 & 172 & & & \\
\hline & 0.83 & 1.43 & 10.6 & 29.5 & 840 & 12.3 & 59.0 & $<0.002$ & 0.03 & 1.10 & & 1.50 & 241.0 & 0.75 & $<0.05$ & 9.6 & 0.424 & 0.36 & 2.4 & & & 17.2 & 116 & 68 & & FA04030730 \\
\hline 588 & 0.79 & 1.37 & 9.6 & 30.7 & 810 & 12.3 & 62.7 & 0.002 & 0.03 & 1.06 & & 1.50 & 231.0 & 0.69 & $<0.05$ & 8.2 & 0.378 & 0.36 & 2.1 & 116 & 1.0 & 15.6 & 94 & 64.3 & & FA04030730 \\
\hline & 1.06 & 1.33 & 10.5 & 29.7 & 790 & 12.1 & 57.4 & $<0.002$ & 0.03 & 1.02 & & 1.5 & 227.0 & \begin{tabular}{|l|}
0.71 \\
\end{tabular} & $<0.05$ & 8.5 & 0.417 & 0.34 & 2.3 & 115 & 1. & 16.6 & 170 & 66.5 & & \\
\hline & 1.08 & 1.36 & 11.2 & 29.1 & 840 & 11.7 & 57.7 & \begin{tabular}{|l|}
0.002 \\
\end{tabular} & 0.02 & 1.02 & & 1.50 & 237.0 & 0.80 & $<0.05$ & 10.2 & 0.455 & 0.35 & 2.7 & 118 & 1. & 18.2 & 106 & 77.1 & 2 & FA04030730 \\
\hline 593 & 1.14 & 1.36 & 10.4 & 32.4 & 840 & 12.9 & 60.9 & $<0.002$ & 0.03 & 1.15 & 2 & 1.50 & 234.0 & \begin{tabular}{|l|}
0.72 \\
\end{tabular} & $<0.05$ & 9.1 & 0.411 & 0.35 & 2.4 & 119 & 1.2 & 17.0 & 182 & 69.2 & 3 & FA04030730 \\
\hline & 0.84 & 1.36 & 12.1 & 29.9 & 900 & 11.8 & 55.6 & $<0.002$ & 0.02 & 1.06 & & 1.60 & 238.0 & \begin{tabular}{|l|}
0.87 \\
\end{tabular} & $<0.05$ & 12.1 & 0.496 & 0.31 & 2.9 & 119 & & \begin{tabular}{|l|l|}
19.4 \\
\end{tabular} & 94 & 04.0 & & \\
\hline & 0.97 & 1.29 & 10.6 & 32.1 & 840 & 12.8 & 56.6 & \begin{tabular}{|l|}
$<0.002$ \\
\end{tabular} & 0.04 & 1.13 & 2 & 1.50 & 224.0 & \begin{tabular}{|l|l|}
0.78 \\
\end{tabular} & $<0.05$ & \begin{tabular}{|l|}
9.5 \\
\end{tabular} & 0.422 & 0.34 & 2.4 & 118 & 1. & \begin{tabular}{|l|l|}
17.4 & \\
\end{tabular} & 329 & 68.9 & 36 & FA04030730 \\
\hline 596 & 0.85 & 1.38 & 12.4 & 30.7 & 900 & 11.5 & 56.0 & $<0.002$ & 0.03 & 1.02 & & 1.60 & 240.0 & \begin{tabular}{|l|l|}
0.88 \\
\end{tabular} & $<0.05$ & 11.7 & 0.519 & 0.32 & 2.8 & 126 & 1.3 & \begin{tabular}{|l|}
20.4 \\
\end{tabular} & 120 & 87.2 & 2 & A04030730 \\
\hline & 1.00 & 1.40 & 11.0 & 32.1 & 870 & 12.7 & 59.6 & & 0.03 & 1.27 & & 1.60 & 248.0 & \begin{tabular}{|l|l|}
0.82 \\
\end{tabular} & $<0.05$ & \begin{tabular}{|l|}
8.8 \\
\end{tabular} & 0.430 & 0.35 & 2.4 & 122 & & 17.9 & 197 & 2.1 & & A04030730 \\
\hline 598 & 0.91 & 1.41 & 10.4 & 29.5 & 790 & 10.8 & 58.1 & $<0.002$ & 0.03 & 1.27 & 1 & 1.60 & 239.0 & 0.70 & $<0.05$ & 7.6 & 0.411 & 0.34 & 2.1 & 117 & 1.1 & 16.0 & 88 & 69.1 & 57 & FA04030730 \\
\hline 600 & 0.93 & 1.42 & 10.8 & 28.3 & 810 & 10.9 & 53.9 & \begin{tabular}{|l|}
$<0.002$ \\
\end{tabular} & 0.02 & 1.02 & 1 & 1.50 & 242.0 & \begin{tabular}{|l|l|}
0.77 \\
\end{tabular} & $<0.05$ & 8.3 & 0.458 & 0.34 & 2.3 & 122 & 1.5 & 17.0 & 86 & 72.3 & 80 & FA04030730 \\
\hline & 0.81 & 1.43 & 11.6 & 27.6 & 840 & 9.9 & 50.5 & $\begin{array}{l}<0.002 \\
\end{array}$ & 0.02 & 0.93 & & 1.50 & 246.0 & 0.87 & $<0.05$ & 9.1 & 0.515 & 0.28 & 2.4 & 123 & 1. & 18.0 & 80 & 80.9 & & \\
\hline 602 & 0.78 & 1.37 & 12.8 & 26.6 & 950 & 9.7 & 49.3 & $<0.002$ & 0.02 & 0.96 & 1 & 1.50 & 245.0 & 0.93 & $<0.05$ & 12.7 & 0.569 & 0.29 & 3.0 & 126 & 1.8 & 20.0 & 101 & 100.0 & 56 & FA04030730 \\
\hline 603 & 0.82 & 1.33 & \begin{tabular}{|l|}
9.9 \\
\end{tabular} & 28.3 & 800 & 10.9 & 52.9 & \begin{tabular}{|c|c|}
$<002$ \\
\end{tabular} & 0.03 & 1.00 & & 1.50 & 229.0 & 0.72 & $<0.05$ & 8.0 & 0.405 & 0.31 & 2.0 & 112 & 0.9 & 16.6 & 98 & 65.6 & 56 & FA04030730 \\
\hline
\end{tabular}


Table 1. Location and New Trace Element Geochemical Results for 902 Stream-Sediment Samples collected in the Livengood Area, Livengood Quadrangle, Alaska IS = Insufficient Sample for Analysis

\begin{tabular}{|c|c|c|c|c|c|c|c|c|c|c|c|c|c|c|c|c|c|c|c|c|c|c|c|c|c|c|c|}
\hline MPLE & TM E & TM N & $\begin{array}{l}\mathrm{Au} \\
\mathrm{ppm}\end{array}$ & $\begin{array}{c}\mathrm{Ag} \\
\mathrm{ppm}\end{array}$ & $\begin{array}{l}\text { Al } \\
\%\end{array}$ & $\begin{array}{l}\text { As } \\
\text { ppm }\end{array}$ & $\begin{array}{l}\mathrm{Ba}^{*} \\
\mathrm{ppm}\end{array}$ & $\begin{array}{c}\mathrm{Be} \\
\mathrm{ppm}\end{array}$ & $\begin{array}{c}\mathrm{Bi} \\
\mathrm{ppm}\end{array}$ & $\begin{array}{c}\mathrm{Ca} \\
\%\end{array}$ & $\begin{array}{c}\mathrm{Cd} \\
\mathrm{ppm}\end{array}$ & $\begin{array}{c}\mathrm{Ce} \\
\mathrm{ppm}\end{array}$ & $\begin{array}{r}\text { Co } \\
\text { ppm }\end{array}$ & $\begin{array}{l}\mathrm{Cr}^{*} \\
\mathrm{ppm}\end{array}$ & $\begin{array}{l}\text { Cs } \\
\text { ppm }\end{array}$ & $\begin{array}{c}\mathrm{Cu} \\
\mathrm{ppm}\end{array}$ & $\begin{array}{c}\mathrm{Fe} \\
\% \\
\end{array}$ & $\begin{array}{c}\mathrm{Ga} \\
\mathrm{ppm}\end{array}$ & $\begin{array}{c}\mathrm{Ge} \\
\mathrm{ppm}\end{array}$ & $\begin{array}{c}\mathrm{Hf} \\
\mathrm{ppm}\end{array}$ & $\begin{array}{l}\mathrm{Hg} \\
\mathrm{ppm}\end{array}$ & $\begin{array}{c}\text { In } \\
\text { ppm }\end{array}$ & $\begin{array}{l}\mathrm{K} \\
\% \\
\end{array}$ & $\begin{array}{r}\mathrm{La} \\
\mathrm{ppm}\end{array}$ & $\begin{array}{c}\mathrm{Li} \\
\mathrm{ppm} \\
\end{array}$ & $\begin{array}{c}\mathrm{Mg} \\
\% \\
\end{array}$ & $\begin{array}{l}\mathrm{Mn} \\
\mathrm{ppm}\end{array}$ \\
\hline 604 & 3783 & 34896 & IS & \begin{tabular}{|l|}
0.07 \\
\end{tabular} & 5.45 & 7.3 & 850 & 1.31 & & 1.84 & \begin{tabular}{|l|l|}
0.22 \\
\end{tabular} & 65.4 & 10.2 & 79 & 1.91 & 17.0 & 2.69 & 12.75 & 0.17 & & 0.10 & 0.038 & \begin{tabular}{|l|}
1.14 \\
\end{tabular} & 31.0 & 21.4 & 0.94 & 476 \\
\hline 07 & & & & & \begin{tabular}{|l}
6.37 \\
\end{tabular} & 3.7 & 710 & & & & \begin{tabular}{|l|l|}
0.18 \\
\end{tabular} & 106.0 & 10 & 63 & 7.30 & 2. & 3.14 & 16.15 & 0.32 & 1.5 & 0.14 & 0.048 & \begin{tabular}{|l|}
1.20 \\
\end{tabular} & 49.7 & 2.2 & \begin{tabular}{|l|}
1.14 \\
\end{tabular} & 74 \\
\hline & & & .005 & 22 & 6.64 & 4.7 & 750 & 19 & 0.17 & 45 & & \begin{tabular}{|l}
133.5 \\
\end{tabular} & 12.6 & 64 & 11.10 & 5.0 & 3.41 & 17.80 & 0.43 & 16 & 0.09 & 0.050 & 1.25 & 62.9 & 3.9 & 1.31 & 500 \\
\hline & & & & & & 2.9 & 90 & & & & & & & & & & & & & & & & & & 9.0 & 1138 & \\
\hline 11 & & 37754 & 007 & .13 & 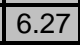 & 24.7 & 780 & .73 & 0.17 & 1.94 & \begin{tabular}{|l|}
0.26 \\
\end{tabular} & 129.5 & 18.9 & 85 & 4.64 & 14.1 & 5.21 & 16.25 & 0.44 & 2.1 & 0.11 & 0.049 & 1.24 & 61.9 & 29.1 & 1.08 & 1130 \\
\hline & & & 005 & 0.14 & \begin{tabular}{|c|}
6.35 \\
\end{tabular} & 19.6 & 750 & 2.01 & 0.16 & 2.37 & \begin{tabular}{|l|}
0.30 \\
\end{tabular} & 153.5 & 19.7 & 73 & 5.61 & 15.1 & 5.51 & 16.90 & 0.46 & 2.0 & 0.10 & 0.057 & 1.20 & 72.9 & \begin{tabular}{|l|}
29.3 \\
\end{tabular} & 1.22 & 1380 \\
\hline & & & & 13 & 7.49 & 63.8 & 570 & & & 2.93 & \begin{tabular}{|l|}
3.68 \\
\end{tabular} & 222.0 & & 50 & 9.47 & 01 & 3.87 & 20.40 & 0.51 & 4.0 & & & \begin{tabular}{|l|}
1.48 \\
\end{tabular} & & & 1.24 & 974 \\
\hline & & & .005 & 95 & \begin{tabular}{|l|l|}
7.14 \\
\end{tabular} & 65.4 & 650 & 2.86 & 0.80 & 2.71 & \begin{tabular}{|l|}
3.18 \\
\end{tabular} & \begin{tabular}{|l|l}
176.0 \\
\end{tabular} & 11. & 57 & 33.70 & 31.1 & 3.40 & 19.35 & 0.43 & 2.0 & 0.20 & 0.077 & 1.48 & 84.1 & 5.8 & 1.14 & 341 \\
\hline & & & & 74 & 7.14 & 10.4 & 940 & 62 & 0.17 & 0.97 & \begin{tabular}{|l|}
1.19 \\
\end{tabular} & 58.8 & 15.2 & 174 & 6.68 & 46.3 & 3.81 & 17.85 & 0.31 & 2.1 & 0.21 & 0.058 & \begin{tabular}{|l|}
1.30 \\
\end{tabular} & 29.0 & 44.1 & 1.31 & 549 \\
\hline & & & 0.039 & 54 & \begin{tabular}{|l|l|}
6.79 \\
\end{tabular} & 6.1 & 50 & .56 & 0.16 & 1.28 & \begin{tabular}{|l|l|}
0.55 \\
\end{tabular} & 63.1 & 14.2 & 164 & 5.12 & 33.2 & 3.33 & 16.40 & 0.31 & 2.3 & 0.21 & 0.049 & \begin{tabular}{|l|l}
1.32 \\
\end{tabular} & 34.4 & 7.6 & 1.12 & 72 \\
\hline & & & 0.007 & 0.41 & 6.62 & 5. & 730 & 1.53 & 0.14 & 1.26 & 0.46 & 62.3 & 13.5 & 168 & 4.69 & 40.8 & 3.08 & 15.95 & 0.29 & 2.1 & 0.22 & 0.047 & \begin{tabular}{|l}
1.32 \\
\end{tabular} & 33.0 & 51.5 & 1.04 & 25 \\
\hline & & & 022 & 39 & \begin{tabular}{|l|l|}
6.25 \\
\end{tabular} & 7. & 760 & 62 & & 1.30 & \begin{tabular}{|l|l|}
0.48 \\
\end{tabular} & 6 & 13.9 & 14 & 4.84 & +7.0 & 3.07 & 16.00 & 0.29 & 2.1 & 0.21 & 0.049 & 1.28 & 30.4 & 43.9 & 0.94 & 510 \\
\hline & & & 0.027 & 30 & 41 & 5. & DO & $\overline{40}$ & & .19 & 40 & 7.8 & 12. & & 3.9 & 3. & 2.69 & 15.70 & 0.25 & 21 & 0.40 & .043 & 1.26 & 8.1 & 4.4 & 0.92 & $\overline{04}$ \\
\hline & & & 0.018 & .21 & 6.33 & 8.3 & 790 & 1.52 & 0.14 & 1.18 & \begin{tabular}{|l|l|}
0.31 \\
\end{tabular} & 61.1 & 14.5 & 146 & 3.52 & 31.5 & 3.32 & 15.60 & \begin{tabular}{|l}
0.27 \\
\end{tabular} & 2.1 & 0.19 & 0.044 & 1.20 & 29.1 & 40.6 & 0.94 & 488 \\
\hline & & & & 16 & 4.96 & & 760 & & & 1.16 & \begin{tabular}{|l|l|}
0.49 \\
\end{tabular} & & 16.6 & & & 10.4 & 7.69 & 12.10 & 0.36 & 1.3 & 0.25 & 040 & 0.96 & 24.5 & 23.6 & 0.69 & 539 \\
\hline & & & 0.051 & 14 & \begin{tabular}{|l|l|}
5.70 \\
\end{tabular} & 26.1 & 830 & .2 & 0.1 & 1.12 & 0.36 & 0.7 & 11. & 7 & 2.51 & 16. & 4.44 & 14.75 & 0.30 & $\overline{1.8}$ & 0.22 & 041 & \begin{tabular}{|l}
1.17 \\
\end{tabular} & 24.8 & 5.7 & & 62 \\
\hline & & & 0.058 & .10 & 5.51 & 10.0 & 760 & .18 & ).12 & 1.18 & .22 & 49.8 & 9. & & 2.15 & 11. & 2.95 & 3.80 & 0.25 & 1.8 & 0.15 & .036 & \begin{tabular}{|l|}
1.10 \\
\end{tabular} & 24.0 & 22.3 & 0.77 & 298 \\
\hline & & & 0.018 & $\overline{10}$ & 5.93 & 8 & & & & 36 & & & 9 & & & & 2.88 & 4.95 & & & & $\overline{44}$ & 1.21 & & 3.8 & 0.88 & \\
\hline & & & .012 & .14 & 6.36 & 8.2 & 900 & 1.42 & 0.16 & 1.44 & 0.23 & 58.6 & 12.2 & 8 & 2.53 & 19.4 & 3.28 & 16.05 & 0.30 & 2.1 & 0.12 & 044 & 1.28 & 28.6 & 27.4 & 0.96 & $\overline{514}$ \\
\hline & & & .005 & 06 & 5.97 & 4. & 810 & - & & 1.36 & .16 & & 8. & & 1.9 & 10. & 2.37 & 14.15 & 0.2 & 1.8 & 0.1 & .038 & \begin{tabular}{|l|l|}
1.18 \\
\end{tabular} & 25.9 & 21.3 & 0.86 & 299 \\
\hline & & & 009 & 07 & & 3 & $\overline{78}$ & & & 38 & & & 8 & & & & & & & & & & 1.20 & & 3.0 & & 31 \\
\hline & & & 0.008 & 0.08 & 6.11 & 5.5 & 840 & 1.36 & 0.14 & 1.46 & \begin{tabular}{|l|l|}
0.23 \\
\end{tabular} & 59.4 & 10.3 & & 2.21 & 16.3 & 2.70 & 14.90 & 0.28 & 2.0 & 0.09 & .041 & \begin{tabular}{|l|}
1.24 \\
\end{tabular} & 28.0 & 24.1 & 0.94 & 372 \\
\hline & & & 0 & 09 & 6.44 & 8 & 910 & & & 1.73 & 0.28 & 6 & 12. & & 2.6 & 21. & 3.40 & 6.30 & 0.3 & 2. & 0.09 & .044 & \begin{tabular}{|l|}
1.37 \\
\end{tabular} & 31.1 & 27.5 & 1.07 & 508 \\
\hline & & & & & & 6 & 670 & & & 28 & & & 9.2 & & & & & & & & & & 0.97 & 21.5 & 6.0 & & 97 \\
\hline & & & 0.015 & 09 & 6.70 & 8. & 950 & $\overline{144}$ & 0.1 & 1.86 & 3.31 & 6. & 13. & & 2.62 & 21. & 3.48 & 16.30 & 0.3 & 2. & 0.06 & 0.045 & 1.40 & 30.7 & 28.4 & 1.09 & 561 \\
\hline & & & & & & 8 & & & & 76 & & & & & & & 3.46 & 5.90 & 0.3 & & & 045 & 1.37 & 28.4 & 9.1 & 1.06 & 548 \\
\hline & & & & 26 & 6.04 & 7. & 760 & 1.20 & & 1.23 & \begin{tabular}{|l|}
3.09 \\
\end{tabular} & & & & 4.67 & 22.5 & 2.74 & 5.65 & 0.30 & 1.7 & 0.54 & 042 & \begin{tabular}{|l|}
1.21 \\
\end{tabular} & 25.4 & 36.3 & 1.02 & 770 \\
\hline & & & & & & 7. & 670 & & & 1.16 & & & 1 & & 4.6 & 14. & 3.09 & 6.35 & 0.3 & 1.9 & 0.25 & 1.046 & \begin{tabular}{|l|}
1.14 \\
\end{tabular} & 25.4 & 39.7 & 1.12 & 769 \\
\hline & & & & 67 & & 9 & & & & & 0.57 & & & & & & 3.3 & .35 & & & & & & 25.3 & 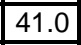 & 1.1 & 766 \\
\hline & & & & & & $\overline{9}$ & & & & & 276 & & & & & & $\sqrt{31}$ & 5,15 & $\sqrt{01}$ & & $\sqrt{01}$ & $\overline{0.5}$ & $\overline{1.2}$ & 27. & 4.9 & 0.93 & 1275 \\
\hline & & & $\overline{040}$ & 92 & 6.12 & - & 710 & & & 93 & & & 26 & & 6.8 & 5. & 3.45 & 15.00 & 0.10 & 1. & 0.44 & .053 & 1.22 & 25.5 & 46.4 & 0.98 & 1830 \\
\hline & & & & 46 & 6.36 & 10.2 & 690 & & $\overline{0 .}$ & 0.60 & 0.34 & 45 & & & & & 3.25 & $\overline{6.20}$ & 0. & & $0 . \angle U$ & & 1.30 & 24.6 & 50.7 & 0.92 & 330 \\
\hline & & & 005 & & 7.31 & 33.1 & 760 & & & & & & & & & & 5.14 & 7.40 & & & 0.76 & 059 & 1.47 & 24.1 & 71.0 & 1.41 & 1985 \\
\hline & & 53 & 0.009 & 0.17 & 6.20 & 5.7 & 800 & 1.32 & 0.1 & 1.42 & \begin{tabular}{|l}
0.23 \\
\end{tabular} & 58.6 & 10.7 & 89 & 3.07 & 19. & 2.75 & 14.85 & 0.10 & 1.7 & 0.20 & 0.050 & 1.23 & 32.0 & 28.5 & 1.00 & 437 \\
\hline & & & & & & 18.3 & & & & & & & & & & & 3.99 & & & & 3.52 & & 1.29 & 29.5 & 3.3 & 1.21 & 1200 \\
\hline & & & & & & 7.6 & 880 & & & & & & & & 12 & & 3.01 & 4.50 & & & 0.51 & .051 & \begin{tabular}{|l|}
1.24 \\
\end{tabular} & 38.4 & 55.0 & 0.86 & 3140 \\
\hline & & 254 & 012 & 0.31 & \begin{tabular}{|l|l|}
5.91 \\
\end{tabular} & 11.5 & 670 & 1.1 & 0. & 1.25 & \begin{tabular}{|l|l|} 
\\
\end{tabular} & $\begin{array}{l}62.7 \\
\end{array}$ & 15. & 116 & 4.5 & & 3.12 & 14.40 & 0.10 & 1.8 & 0.19 & .050 & \begin{tabular}{|l|}
1.21 \\
\end{tabular} & 33.1 & 34.7 & 1.05 & 962 \\
\hline & & & & & & & 770 & & & & & & & & & & & & & & 80 & & & & 0.1 & 1.15 & 2630 \\
\hline & & & .005 & 0.55 & \begin{tabular}{|l}
6.55 \\
\end{tabular} & 5.8 & 770 & & 0. & 1.06 & & 53.1 & 1 & & 7.26 & $\begin{array}{l}32.6 \\
\end{array}$ & 3.76 & 15.75 & 0 & 1.6 & 0.38 & 0.109 & \begin{tabular}{|l|l|}
1.37 \\
\end{tabular} & 26.7 & 88.4 & 1.09 & 1890 \\
\hline & & & 0.026 & 0.39 & \begin{tabular}{|l|l|}
6.49 \\
\end{tabular} & 11.6 & 810 & 1. & 0. & 1.35 & \begin{tabular}{|l|l|}
0.45 \\
\end{tabular} & 70 & 13 & 132 & 4.9 & 24.4 & 3.28 & 15.60 & 0.12 & 2.0 & 0.33 & 0.073 & \begin{tabular}{|l|l}
1.33 \\
\end{tabular} & 37.9 & 30.2 & \begin{tabular}{|l}
1.03 \\
\end{tabular} & 770 \\
\hline & & & & & & $10 . C$ & 880 & & & & & & & & & & 3.2 & & & 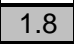 & 0.24 & & \begin{tabular}{|l}
1.32 \\
\end{tabular} & 34.6 & 6 & 1.02 & 1110 \\
\hline 764 & & 7258 & 0.00 & 0.41 & 6.34 & 9.2 & 850 & 1. & 0.19 & 1.53 & 0.75 & 63. & 15. & 10 & 3.9 & 23. & 3.11 & 14.90 & & 1.7 & 0.31 & $.05 \varepsilon$ & 1.34 & 33.1 & 42.5 & 0.99 & 1355 \\
\hline & & & 0.017 & 0.40 & 10.0 & 9.9 & 750 & & 0.20 & 1.40 & 0.41 & 10.0 & 12.1 & 110 & 1.00 & & 2.96 & & & & 0.25 & 0.082 & 1.27 & 38.5 & & 0.98 & 126 \\
\hline
\end{tabular}


Table 1. Location and New Trace Element Geochemical Results for 902 Stream-Sediment Samples collected in the Livengood Area, Livengood Quadrangle, Alaska IS = Insufficient Sample for Analysis

\begin{tabular}{|c|c|c|c|c|c|c|c|c|c|c|c|c|c|c|c|c|c|c|c|c|c|c|c|c|c|c|}
\hline SAMPLE & $\begin{array}{l}\text { Mo } \\
\text { ppm }\end{array}$ & $\begin{array}{c}\mathrm{Na} \\
\%\end{array}$ & $\begin{array}{c}\mathrm{Nb} \\
\mathrm{ppm}\end{array}$ & $\begin{array}{c}\mathrm{Ni} \\
\mathrm{ppm}\end{array}$ & $\begin{array}{c}\mathrm{P} \\
\mathrm{ppm}\end{array}$ & $\begin{array}{l}\mathrm{Pb} \\
\mathrm{ppm}\end{array}$ & $\begin{array}{l}\mathrm{Rb} \\
\mathrm{ppm}\end{array}$ & $\begin{array}{l}\mathrm{Re} \\
\mathrm{ppm}\end{array}$ & $\begin{array}{l}\mathrm{S} \\
\%\end{array}$ & $\begin{array}{l}\text { Sb } \\
\text { ppm }\end{array}$ & $\begin{array}{c}\text { Se } \\
\text { ppm }\end{array}$ & $\begin{array}{l}S^{S} n^{*} \\
\text { ppm }\end{array}$ & $\begin{array}{c}\mathrm{Sr} \\
\mathrm{ppm}\end{array}$ & $\begin{array}{l}\mathrm{Ta}^{*} \\
\mathrm{ppm}\end{array}$ & $\begin{array}{c}\text { Te } \\
\text { ppm }\end{array}$ & $\begin{array}{c}\text { Th } \\
\text { ppm }\end{array}$ & $\begin{array}{l}\mathrm{Ti}^{*} \\
\%\end{array}$ & $\begin{array}{c}\mathrm{Tl} \\
\mathrm{ppm}\end{array}$ & $\begin{array}{c}U \\
\mathrm{ppm}\end{array}$ & $\begin{array}{c}\mathrm{V} \\
\mathrm{ppm}\end{array}$ & $\begin{array}{l}\mathrm{W}^{*} \\
\mathrm{ppm}\end{array}$ & \begin{tabular}{|c|}
$\mathrm{Y}$ \\
$\mathrm{ppm}$
\end{tabular} & $\begin{array}{c}\mathrm{Zn} \\
\mathrm{ppm}\end{array}$ & $\begin{array}{l}\mathrm{Zr}^{*} \\
\mathrm{ppm}\end{array}$ & $\begin{array}{c}\text { Pulp Wt } \\
\text { grams }\end{array}$ & $\begin{array}{c}\text { Lab } \\
\text { Report }\end{array}$ \\
\hline 604 & 0.69 & 1.42 & 10.6 & 25.2 & 810 & 10.8 & 46.0 & 0.002 & 0.02 & 0.96 & 1 & 1.40 & 237.0 & 0.88 & $<0.05$ & 8.4 & 0.426 & 0.28 & 2.3 & 108 & 1.3 & 17.0 & 85 & 68.3 & 53 & FA04030730 \\
\hline 607 & 0.45 & 44 & 18.7 & 18.9 & 1750 & 15.6 & 83.4 & $<0.002$ & 0.02 & 0.89 & 1 & 2.30 & 293.0 & 1.32 & $<0.05$ & 19.4 & 0.435 & 0.45 & 6.3 & 106 & 1.2 & 18.1 & 108 & 45.1 & 22 & \\
\hline 608 & .57 & .49 & 22.8 & 20.1 & 2310 & 18.0 & 91.1 & $<0.002$ & 0.03 & 1.14 & 1 & 2.70 & 374.0 & 1.46 & $<0.05$ & 19.5 & 0.491 & 0.54 & 6.2 & 108 & 1.7 & 22.2 & 90 & 46.5 & 3 & A04030730 \\
\hline 610 & 0.39 & 1.74 & 29.3 & 19.6 & 2940 & 14.2 & 77.2 & $<0.002$ & 0.01 & 1.06 & & 3.40 & 405.0 & 2.00 & $<0.05$ & 23.4 & 0.509 & 0.41 & 5.2 & 106 & 1.4 & 28.5 & 136 & 50.0 & & FA0403 \\
\hline & 80 & 31 & 20.3 & 25.0 & 2220 & 14.5 & 74.8 & $<0.002$ & 0.03 & 1.11 & 1 & 2.30 & 272.0 & 1.40 & $<0.05$ & 18.1 & 0.497 & 0.45 & 5.1 & 140 & 15 & 22.1 & 100 & 69.7 & & \\
\hline 612 & 0.83 & 1.41 & 30.9 & 22.6 & 2740 & 15.6 & 77.9 & $<0.002$ & 0.02 & 1.12 & 2 & 3.10 & 333.0 & 2.20 & $<0.05$ & 33.3 & 0.543 & 0.48 & 8.7 & 143 & 1.9 & 27.0 & 133 & 57.7 & & FA04030730 \\
\hline & & 97 & 49.9 & 14.9 & 3550 & 103.0 & 124.5 & $<0.002$ & 0.03 & 3.69 & & 7.50 & 521.0 & 4.03 & $<0.05$ & 32.2 & 0.629 & 0.65 & 13.4 & 118 & 2.8 & $\begin{array}{ll}33.8 \\
\end{array}$ & 255 & 125.5 & & 0730 \\
\hline$\overline{16}$ & 62 & .84 & 32.6 & 19.1 & 2940 & 91.6 & 129.5 & $<0.002$ & 0.04 & 3.37 & 2 & 6.30 & 578.0 & 2.38 & $<0.05$ & 25.1 & 0.481 & 1.10 & 11.4 & 107 & 8.7 & \begin{tabular}{|l|}
28.5 \\
\end{tabular} & 373 & 61.3 & & $\overline{\text { FA0403 }}$ \\
\hline 701 & 1.26 & 0.77 & 7.8 & 121.5 & 990 & 14.6 & 83.0 & $<0.002$ & 0.05 & 1.32 & & 2.00 & 152.5 & 0.53 & $<0.05$ & 7.3 & 0.327 & 0.61 & 2.9 & 166 & 1.0 & 21.0 & 162 & 72.5 & & FA04030730 \\
\hline 3 & 00 & 79 & 7.6 & 101.5 & 710 & 12.6 & 69.4 & $<0.002$ & 0.07 & 1.58 & & 1.80 & 203.0 & 0.52 & $<0.05$ & 8.1 & 0.348 & 0.52 & 3.5 & 154 & & \begin{tabular}{|l|}
22.4 \\
\end{tabular} & 190 & 79.5 & & 0730 \\
\hline$\overline{54}$ & 90 & .79 & 7.5 & 97.0 & 620 & 11.3 & 66.9 & $<0.002$ & 0.08 & 1.10 & 2 & 1.70 & 199.5 & 0.50 & $<0.05$ & 7.7 & 0.358 & 0.49 & 3.2 & 150 & 10 & 19.9 & 275 & 73.5 & & 730 \\
\hline 705 & 1.09 & 0.71 & 7.9 & 91.1 & 630 & 12.0 & 67.6 & $<0.002$ & 0.09 & 1.30 & 2 & 1.70 & 198.0 & 0.54 & $<0.05$ & 7.7 & 0.342 & 0.51 & 3.6 & 148 & 1.0 & 21.0 & 251 & 74.1 & & FA04030730 \\
\hline & & .93 & 8.1 & 70.6 & 530 & 11.5 & 64.9 & $<0.002$ & 0.11 & 1.12 & & 2.00 & 190.5 & 0.53 & $<0.05$ & 7.7 & 0.354 & 0.48 & 2.7 & 134 & & \begin{tabular}{|l|}
16.4 \\
\end{tabular} & 268 & 71.1 & & \\
\hline 707 & 1.02 & 0.96 & 8.3 & 73.9 & 620 & 12.6 & 63.5 & $<0.002$ & 0.06 & 1.25 & 2 & 1.70 & 189.5 & 0.58 & $<0.05$ & 8.3 & 0.362 & 0.43 & 2.9 & 135 & 1.0 & 17.1 & 95 & 68.2 & & FA040 \\
\hline 727 & 2.09 & 0.90 & 7.5 & 27.3 & 1700 & 14.3 & 47.5 & $<0.002$ & 0.06 & 1.36 & & 1.60 & 166.0 & 0.55 & $<0.05$ & 6.4 & 0.311 & 0.28 & 1.9 & 132 & 0.9 & 14.8 & 84 & 55.8 & & FA04030730 \\
\hline & & 1.08 & 8.9 & 27.7 & 1200 & 15.8 & 60.3 & $<0.002$ & 0.05 & 1.50 & & 1.80 & 179.5 & 0.61 & $<0.05$ & 7.2 & 0.364 & 0.35 & 2.1 & 126 & & 14.2 & 97 & 61.8 & & \\
\hline 730 & .13 & 1.18 & 9.0 & 22.7 & 800 & 12.4 & 56.9 & $<0.002$ & 0.04 & 1.08 & & 1.70 & 185.5 & 0.64 & $<0.05$ & 6.8 & 0.369 & 0.31 & 2.0 & 104 & 1. & 14.0 & 8 & 59.3 & & FA04 \\
\hline & 02 & 1.31 & 10.2 & 24.0 & 760 & 12.7 & 57.8 & $<0.002$ & 0.03 & 1.18 & & 1.70 & 206.0 & 0.73 & $<0.05$ & 8.3 & 0.423 & 0.33 & 2.1 & 113 & & \begin{tabular}{|l|}
14.2 \\
\end{tabular} & & 63. & & 730 \\
\hline & 0.86 & 1.32 & 10.2 & 27.9 & 760 & 13.2 & 62.7 & 0.002 & 0.03 & 1.18 & 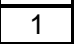 & 2.00 & 210.0 & 0.72 & $<0.05$ & 8.5 & 0.433 & 0.40 & 2.4 & & & 15.4 & 104 & 68. & & 7730 \\
\hline 733 & 0.32 & 1.47 & 9.9 & 20.7 & 520 & 11.2 & 51.1 & $<0.002$ & 0.01 & 1.06 & & 1.50 & 219.0 & 0.71 & $<0.05$ & 7.3 & 0.413 & 0.30 & 2.0 & 108 & 1.1 & 13.4 & 63 & 60.5 & & FA04030730 \\
\hline 7 & 55 & 1.36 & 10.8 & 21.7 & 600 & 11.7 & 55.3 & $<0.002$ & 0.02 & 1.02 & & 1.60 & 210.0 & 0.76 & $<0.05$ & 8.6 & 0.452 & 0.32 & 2.3 & 112 & & 14.2 & & 67.0 & & FA040 \\
\hline & 53 & 37 & 10.1 & 26.1 & 680 & 11.7 & 57.7 & $<0.002$ & 0.03 & 1.06 & & 1.70 & 216.0 & 0.70 & $<0.05$ & 8.1 & 0.420 & 0.35 & 2.2 & 114 & & 15.2 & 91 & 64. & & $\mathrm{FAO}$ \\
\hline & .94 & 1.37 & 11.1 & 30.6 & 860 & 13.0 & 63.1 & $<0.002$ & 0.03 & 1.24 & & 1.80 & 227.0 & 0.81 & $<0.05$ & 9.2 & 0.455 & 0.36 & 2.4 & 128 & & 17.1 & 124 & 70.9 & & FA04030730 \\
\hline & 68 & 0.98 & 7.8 & 21.7 & 620 & 9.2 & 45.3 & 0.002 & 0.03 & 0.88 & & 1.30 & 162.5 & 0.55 & $<0.05$ & 6.5 & 328 & 0.27 & 1.7 & 92 & & 12.2 & 72 & 51. & & 730 \\
\hline & 96 & 1.45 & 11.0 & 30.3 & 850 & 13.0 & 63.9 & $<0.002$ & 0.03 & 1.24 & 2 & 1.70 & 238.0 & 0.75 & $<0.05$ & 9.4 & 0.451 & 0.39 & 2.3 & 128 & 1.2 & 17.1 & 124 & 70.2 & & FA04 \\
\hline & .95 & 1.38 & 10.6 & 29.3 & 840 & 12.9 & 63.3 & $<0.002$ & 0.04 & 1.24 & & 1.90 & 226.0 & 0.76 & $<0.05$ & 8.6 & 0.444 & 0.35 & 2.2 & 127 & & \begin{tabular}{|l|}
16.2 \\
\end{tabular} & 122 & 65.9 & & 0730 \\
\hline & & .10 & 9.1 & 32.5 & 830 & 60.8 & 66.4 & $<0.002$ & 0.07 & 6.63 & & 2.80 & 171.0 & 0.65 & $<0.05$ & 6.6 & 0.415 & 0.40 & 1.8 & 12 & & 16. & 342 & 56 & & 7730 \\
\hline & & .28 & 9.7 & 30.5 & 570 & 33.4 & 63.3 & 0.002 & 0.03 & 3.52 & & 2.10 & 163.0 & 0.69 & $<0.05$ & 7.3 & 0.485 & 0.38 & 1.8 & 136 & & \begin{tabular}{|l|l|}
13.7 \\
\end{tabular} & 13 & 63 & & FA04 \\
\hline & .86 & 1.27 & 10.1 & 32.6 & 610 & 32.5 & 63.3 & $<0.002$ & 0.03 & 3.08 & & 2.10 & 150.0 & 0.68 & $<0.05$ & 6.8 & 0.506 & 0.40 & 1.8 & 144 & & 13.6 & 12 & 60.4 & & 7730 \\
\hline & & 1.26 & 10.0 & 28.4 & 610 & 38.0 & 65.5 & $<0.002$ & 0.03 & 3.43 & & 2.00 & 172.0 & 0.73 & $<0.05$ & 6.6 & 0.455 & 0.42 & 1.8 & 133 & & 14.0 & 123 & 68 & & \\
\hline & 24 & 1.06 & 10.3 & 33.4 & 840 & 102.0 & 69.5 & 0.002 & 0.04 & 10.10 & & 5.30 & 143.5 & 0.73 & \begin{tabular}{|l|}
0.05 \\
\end{tabular} & 6.2 & 0.465 & 0.44 & 1.8 & 148 & & \begin{tabular}{|l|}
15.2 \\
\end{tabular} & 230 & 66.5 & & 730 \\
\hline & $\overline{00}$ & 0.92 & 9.7 & 33.0 & 700 & 31.0 & 84.6 & $<0.002$ & 0.04 & 1.84 & $<1$ & 2.20 & 111.0 & 0.72 & $<0.05$ & 6.4 & 0.439 & 0.55 & 1.8 & 152 & & 12.4 & 118 & 66.9 & & FA0403 \\
\hline & & & 10.3 & & 980 & 68.5 & 74.8 & & 0.03 & 5.88 & & 42.80 & & 0.77 & \begin{tabular}{|l|}
0.06 \\
\end{tabular} & 6.0 & & 0.48 & 1.9 & 196 & & & 246 & & & \\
\hline & 0.56 & 1.30 & 10.6 & 28.9 & 640 & 15.3 & 62.5 & $<0.002$ & 0.03 & 1.31 & $<1$ & 2.00 & 203.0 & 0.84 & $<0.05$ & 8.9 & 0.440 & 0.36 & 2.1 & 116 & & 16.2 & 88 & 67.2 & & FA04030730 \\
\hline 755 & 1.06 & 1.22 & 10.6 & 44.6 & 820 & 34.3 & 67.1 & $<0.002$ & 0.03 & 6.03 & 1 & 6.80 & 148.0 & 0.78 & $<0.05$ & 7.2 & 0.496 & 0.42 & 2.0 & 155 & 1.5 & 17.4 & 169 & 66.3 & & 0730 \\
\hline & & & \begin{tabular}{|l|}
8.3 \\
\end{tabular} & & 980 & & & & 0.09 & & & & & & & & & & & & & & & 66.2 & & \\
\hline 758 & 0.76 & 1.34 & 11.3 & 30.9 & 690 & 23.4 & 58.4 & $<0.002$ & 0.03 & 3.03 & $<1$ & 1.80 & 177.0 & 0.86 & $<0.05$ & 8.9 & 0.541 & 0.36 & 2.1 & 135 & 1.3 & 15.4 & 112 & 73.4 & & FA04030730 \\
\hline 759 & 0.90 & 1.30 & 10.9 & 40.8 & 770 & 23.2 & 65.7 & $<0.002$ & 0.03 & 2.60 & 1 & 1.90 & 174.5 & 0.81 & $<0.05$ & 7.8 & 0.552 & 0.42 & 2.1 & 160 & & 18.1 & 182 & 73.5 & & \\
\hline & 1.02 & 1.11 & 10.0 & 41.2 & 850 & 27.3 & 81.8 & $<0.002$ & 0.04 & 2.44 & $<1$ & & 150.5 & 0.73 & $<0.05$ & 7.0 & 0.456 & 0.56 & 2.0 & 146 & & & 124 & 70.0 & & $\mathrm{FAO} 2$ \\
\hline 761 & 1.00 & 1.27 & 12.2 & 34.8 & 740 & 33.5 & 69.5 & $<0.002$ & 0.03 & 2.56 & 1 & 2.40 & 191.0 & 0.92 & $<0.05$ & 10.0 & 0.535 & 0.47 & 2.5 & 140 & 1.3 & \begin{tabular}{|l|}
18.1 \\
\end{tabular} & 161 & 86.5 & 2 & FA04030730 \\
\hline & 0.95 & 1.28 & 11.8 & 35.9 & 820 & 27.7 & 69.8 & & 0.05 & 1.63 & & 2.30 & 207.0 & 0.87 & $<0.05$ & 10.1 & 0.459 & 0.48 & 2.4 & 131 & 1. & 17.8 & 156 & 70.3 & & \\
\hline 764 & 0.95 & 1.30 & 10.8 & 35.2 & 780 & 26.6 & 69.3 & $<0.002$ & 0.04 & 1.57 & $<1$ & 2.90 & 206.0 & 0.82 & $<0.05$ & 9.1 & 0.483 & 0.44 & 2.2 & 132 & 1.3 & 17.4 & 133 & 72.1 & 2 & FA04030730 \\
\hline $10 J$ & 0.84 & 1.36 & 11.8 & \begin{tabular}{|l|}
31.8 \\
\end{tabular} & 690 & 61.2 & 66.7 & $<0.002 \mid$ & 0.03 & 3.27 & & 3.00 & 207.0 & 0.86 & $<0.05$ & 9.7 & 0.484 & 0.41 & 2.3 & 124 & 1.6 & 17.7 & 128 & 75.7 & 44 & FA04030730 \\
\hline
\end{tabular}


Table 1. Location and New Trace Element Geochemical Results for 902 Stream-Sediment Samples collected in the Livengood Area, Livengood Quadrangle, Alaska IS = Insufficient Sample for Analysis

\begin{tabular}{|c|c|c|c|c|c|c|c|c|c|c|c|c|c|c|c|c|c|c|c|c|c|c|c|c|c|c|c|}
\hline MPLE & TM E & TM N & $\begin{array}{l}\mathrm{Au} \\
\mathrm{ppm}\end{array}$ & $\begin{array}{c}\mathrm{Ag} \\
\mathrm{ppm}\end{array}$ & $\begin{array}{l}\text { Al } \\
\%\end{array}$ & $\begin{array}{l}\text { As } \\
\text { ppm }\end{array}$ & $\begin{array}{l}\mathrm{Ba}^{*} \\
\mathrm{ppm}\end{array}$ & \begin{tabular}{|c|}
$\mathrm{Be}$ \\
$\mathrm{ppm}$ \\
\end{tabular} & $\begin{array}{c}\mathrm{Bi} \\
\mathrm{ppm}\end{array}$ & $\begin{array}{c}\mathrm{Ca} \\
\%\end{array}$ & \begin{tabular}{|c|}
$\mathrm{Cd}$ \\
$\mathrm{ppm}$ \\
\end{tabular} & $\begin{array}{c}\mathrm{Ce} \\
\mathrm{ppm}\end{array}$ & $\begin{array}{l}\text { Co } \\
\text { ppm }\end{array}$ & $\begin{array}{l}\mathrm{Cr}^{*} \\
\mathrm{ppm}\end{array}$ & $\begin{array}{l}\text { Cs } \\
\text { ppm }\end{array}$ & $\begin{array}{l}\mathrm{Cu} \\
\mathrm{ppm}\end{array}$ & $\begin{array}{c}\mathrm{Fe} \\
\% \\
\end{array}$ & $\begin{array}{c}\mathrm{Ga} \\
\mathrm{ppm} \\
\end{array}$ & $\begin{array}{c}\mathrm{Ge} \\
\mathrm{ppm}\end{array}$ & $\begin{array}{c}\mathrm{Hf} \\
\mathrm{ppm}\end{array}$ & $\begin{array}{l}\mathrm{Hg} \\
\mathrm{ppm}\end{array}$ & $\begin{array}{c}\text { In } \\
\text { ppm }\end{array}$ & $\begin{array}{l}\mathrm{K} \\
\% \\
\end{array}$ & $\begin{array}{c}\mathrm{La} \\
\mathrm{ppm}\end{array}$ & $\begin{array}{c}\mathrm{Li} \\
\mathrm{ppm} \\
\end{array}$ & $\begin{array}{c}\mathrm{Mg} \\
\% \\
\end{array}$ & $\begin{array}{l}\mathrm{Mn} \\
\mathrm{ppm}\end{array}$ \\
\hline 767 & 2308 & 58445 & & 0.37 & & 10.8 & 780 & 1.30 & 0.19 & & 0.59 & & 14.3 & & & 22.3 & 3.23 & & 0.12 & \begin{tabular}{|l}
1.6 \\
\end{tabular} & 0.32 & 0.076 & \begin{tabular}{|l}
1.29 \\
\end{tabular} & 33.1 & 55.8 & 1.00 & 1075 \\
\hline 99 & & & & 14 & \begin{tabular}{|l}
5.51 \\
\end{tabular} & 9.3 & 500 & & $\overline{0.2}$ & & \begin{tabular}{|l|}
0.31 \\
\end{tabular} & 51.3 & 21. & & 3.22 & 3.9 & 3.20 & 15.05 & 0.09 & 1.8 & 0.15 & 0.051 & \begin{tabular}{|l}
1.28 \\
\end{tabular} & 26.7 & 7.4 & \begin{tabular}{|l|l}
0.66 \\
\end{tabular} & 1230 \\
\hline & & & .005 & 16 & 5.25 & 8.6 & 660 & .08 & 0.19 & 88 & .19 & 54.2 & 10.5 & 75 & 2.73 & 29.7 & 2.75 & 3.10 & 0.10 & 1.8 & 0.12 & 0.042 & \begin{tabular}{|l|}
1.08 \\
\end{tabular} & 28.7 & 6.5 & 0.67 & 512 \\
\hline & & & & 19 & \begin{tabular}{|l}
5.91 \\
\end{tabular} & 11.2 & 730 & & & & & & & & 3.33 & 37 & & 5.05 & & & 0.19 & & 1.28 & 30.8 & 9.6 & \begin{tabular}{|l|l|} 
\\
\end{tabular} & 627 \\
\hline 34 & & & .011 & 0.15 & 5.92 & 8.9 & 810 & 1.36 & 0.22 & .14 & 0.41 & 65.5 & 17.1 & 8 & 3.36 & 29.3 & 2.98 & 15.15 & 0.11 & 1.9 & 0.61 & 0.047 & 1.35 & 34.7 & 9.6 & \begin{tabular}{|l}
0.76 \\
\end{tabular} & 1620 \\
\hline & & & 011 & 0.13 & 5.75 & 8.7 & 850 & 1.32 & 0.17 & 1.50 & 0.35 & 61.9 & 11.5 & & 2.57 & 23.2 & 2.96 & 14.05 & 0.11 & 1.8 & 0.12 & 0.043 & 1.20 & 33.9 & 18.8 & 0.85 & 674 \\
\hline & & & & 0.16 & 5.73 & 8.9 & 810 & 1.28 & & & 0.36 & & 13.9 & & 2.96 & 28.8 & 2.95 & 13.65 & 0.09 & 18 & 0.22 & 0.043 & 1.23 & & 18.1 & \begin{tabular}{|l|l}
0.77 \\
\end{tabular} & 926 \\
\hline & & & 0.010 & 0.14 & \begin{tabular}{|l|l|}
6.17 \\
\end{tabular} & 10.4 & 930 & 1.38 & 0.20 & 1.32 & \begin{tabular}{|l|}
0.40 \\
\end{tabular} & 60.4 & 13.1 & 8 & 2.86 & 28.3 & 3.10 & 15.10 & 0.11 & 1.8 & 0.15 & 0.049 & \begin{tabular}{|l|}
1.26 \\
\end{tabular} & 32.9 & 21.8 & 0.86 & 387 \\
\hline & & & .005 & 15 & 6.38 & 11.4 & 1000 & 1.50 & 0.20 & 1.58 & 0.32 & 64.7 & 15.2 & & 3.17 & 25.7 & 3.41 & 15.80 & 0.11 & 1.9 & 0.12 & 0.053 & 1.34 & 35.3 & 21.5 & 1.00 & 943 \\
\hline & & & 005 & 12 & 6.08 & 8.7 & 930 & 1.34 & 0.14 & 1.73 & \begin{tabular}{|l|}
0.31 \\
\end{tabular} & 67.1 & 12. & & 2.48 & 19.1 & 3.02 & 14.35 & 0.12 & 2.0 & 0.19 & 0.047 & 1.29 & 35.7 & 20.0 & 0.93 & 84 \\
\hline & & & 0.005 & 0.11 & 5.96 & 8.1 & 910 & 1.34 & 0.14 & 1.64 & 0.28 & 58.4 & 12.1 & 8 & 2.48 & 17.4 & 2.87 & 14.15 & 0.10 & 1.8 & 0.17 & 0.041 & 1.25 & 32.7 & 18.8 & 0.90 & 718 \\
\hline & & & 007 & 14 & 5.98 & 8.2 & 910 & 1.42 & 0.14 & 1.63 & \begin{tabular}{|l|}
0.29 \\
\end{tabular} & 05.9 & 12.5 & & 2.54 & 17.5 & 2.86 & 14.20 & 0.10 & 1.1 & 0.24 & 0.047 & 1.29 & 31.1 & 19.4 & 0.90 & 727 \\
\hline & & & .005 & 15 & \begin{tabular}{|l|l|}
7.19 \\
\end{tabular} & 10.5 & 100 & 69 & & 2.01 & 32 & 1.4 & 15.2 & & 3.15 & 2. & 3.51 & 18.20 & 0.13 & 2. & 0.26 & .055 & 1.60 & 43.3 & 2.9 & 1.10 & 31 \\
\hline & & & 646 & 0.24 & 5.11 & 10.8 & 910 & 1.20 & 0.16 & 1.24 & 0.35 & 59.9 & 11.0 & 112 & 4.01 & 23.4 & 2.49 & 12.75 & 0.10 & 1.8 & 0.31 & 0.040 & 1.04 & 32.7 & 17.6 & 0.73 & 693 \\
\hline & & & & 0.32 & & & 970 & & & 1.19 & \begin{tabular}{|l|}
0.41 \\
\end{tabular} & & 13.1 & & 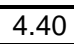 & 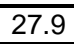 & 2.56 & 12.60 & 0.10 & 1.8 & 0.39 & 0.042 & \begin{tabular}{|l}
1.09 \\
\end{tabular} & 31.0 & 19.6 & \begin{tabular}{|l|l|}
0.71 \\
\end{tabular} & 351 \\
\hline & & & 0.017 & 0.20 & 4.08 & 111.7 & 730 & 0.94 & 0.1 & 2.51 & 5.43 & 38.8 & 9.0 & 65 & $\overline{4.3}$ & 39. & 2.22 & 9.38 & 0.10 & 1.1 & 0.9 & 0.035 & 0.79 & 21.8 & 9.8 & 0.83 & 574 \\
\hline & & & 0.008 & 0.20 & 3.62 & 10.9 & 690 & 0.98 & 0.12 & 2.54 & \begin{tabular}{|l}
5.68 \\
\end{tabular} & 36.8 & 8. & & 3.93 & 41. & 1.97 & 8.87 & 0.09 & 1.0 & 0.91 & .032 & \begin{tabular}{|l|}
0.72 \\
\end{tabular} & 20.7 & 19.8 & 0.77 & 712 \\
\hline & & & 0.005 & 16 & 6.12 & 16.7 & 70 & & & 26 & & & 14. & & & & 3.24 & 14.15 & 0.1 & & 0.22 & 048 & 1.37 & 31.3 & 30.1 & 1.03 & 42 \\
\hline & & & 0.010 & 0.14 & 5.68 & 8.5 & 710 & 1.17 & 0.18 & 1.09 & \begin{tabular}{|l|}
0.23 \\
\end{tabular} & 59.3 & 10.6 & 84 & 3.16 & 17. & 2.55 & 13.95 & 0.10 & 1.8 & 0.11 & 046 & \begin{tabular}{|l|}
1.27 \\
\end{tabular} & 31.8 & 6.8 & \begin{tabular}{|l|l|}
0.74 \\
\end{tabular} & 586 \\
\hline & & & 024 & 14 & 5.52 & 9. & 720 & & & 1.16 & .28 & & 12.2 & & 2.8 & 19. & 2.67 & 13.30 & 0.1 & $1.7 \mathrm{r}$ & 0.19 & .042 & 1.20 & 29.4 & 15.6 & 0.75 & 384 \\
\hline & & & 019 & 15 & 5.50 & 9.4 & 750 & & 0.17 & 16 & 23 & & 12. & & 3.0 & & 2.61 & 4.25 & & 1.7 & & .042 & 1.24 & & 7.1 & & 89 \\
\hline & & & 0.007 & 0.20 & 5.75 & 9.2 & 760 & 1.00 & 0.19 & 0.74 & 0.19 & 49.3 & 7.5 & & 3.39 & 14.4 & 2.69 & 15.05 & 0.14 & 1.8 & 0.20 & .043 & \begin{tabular}{|l|}
1.16 \\
\end{tabular} & 27.4 & 25.4 & 0.67 & 266 \\
\hline & & & $\overline{011}$ & 21 & \begin{tabular}{|l}
6.60 \\
\end{tabular} & 1 & 710 & 1 & & 1.54 & 0.53 & 62. & 25. & & 4.1 & 32. & 3.68 & 15.90 & 0.16 & 1.8 & 0.44 & .055 & 1.22 & 32.2 & 52.0 & 0.88 & 1135 \\
\hline & & & & & 6.53 & & 750 & & & 1.46 & 0.42 & 60.4 & 16. & & 30 & & 3.11 & & & 2. & & & 1.27 & 30.9 & 8.5 & 0.85 & 868 \\
\hline & & & 0.005 & 0.42 & 6.45 & 17.6 & 800 & 1. & & 1.09 & 0.36 & & 17 & & 48 & 27. & 3.52 & 16.05 & 0.17 & 2 & 0.76 & 0.057 & \begin{tabular}{|l|}
1.37 \\
\end{tabular} & 38.1 & \begin{tabular}{|l|}
54.3 \\
\end{tabular} & 0.68 & 958 \\
\hline & & & 008 & 0.06 & & 9.1 & 6 & & & 1.30 & 16 & $54 . !$ & & & & 4. & 3.47 & 4.25 & 0.18 & 2.1 & .10 & .048 & 0.94 & 28.4 & 9.4 & \begin{tabular}{|l|l|}
0.78 \\
\end{tabular} & 456 \\
\hline & & & .005 & 0.06 & 5.86 & 10.9 & 620 & 0.99 & & 1.22 & 0.22 & 50.4 & & & 2,23 & 28.7 & 3.66 & 5.30 & 0.17 & 2.0 & 14 & 051 & \begin{tabular}{|l|}
0.98 \\
\end{tabular} & 27.3 & 0.9 & \begin{tabular}{|l|} 
\\
\end{tabular} & 429 \\
\hline & & & 005 & & 6.50 & 8.3 & 720 & & & 1.20 & 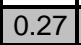 & 54 & 13. & & 2.8 & 31.6 & 3.12 & 5.45 & 0 & 2.0 & 0.26 & 05 & \begin{tabular}{|l|}
1.04 \\
\end{tabular} & 28.6 & 27.7 & 0.74 & 505 \\
\hline & & & 005 & & 6.11 & & 870 & & & & & & & & & & & .05 & & & & & 1.1 & 31 & 49.7 & 0.78 & 19 \\
\hline & & & $\overline{005}$ & & & & 690 & & & & & & & & & & $\sqrt{13}$ & $=10$ & & & $\sqrt{16}$ & $\overline{06}$ & & 36 & 5.1 & 0.51 & 685 \\
\hline & & & .005 & 12 & 6.25 & 3.9 & 690 & & 0.24 & .58 & & 0 & & & 4.8 & 0.5 & 4.27 & 6.50 & 0.21 & 1.9 & 0.71 & .070 & 1.55 & 41.3 & 7.7 & 0.50 & 675 \\
\hline & & & 005 & 25 & 5.50 & 25.7 & 600 & 1. & 0. & 0.67 & 0.39 & $\overline{61}$ & & & & & 4.05 & 3.50 & & 2.1 & 0.54 & & 1.28 & 29.4 & 59.2 & 0.50 & 591 \\
\hline & & & n08 & & \begin{tabular}{|l|}
6.46 \\
\end{tabular} & 25.7 & 1000 & & & & & & & & & & 3.52 & 1.10 & & & 0.44 & .052 & 1.28 & 42.6 & 6.5 & 0.84 & 575 \\
\hline & & 6 & 009 & 0.39 & 6.30 & 27.6 & 710 & 1.64 & 0.22 & 0.79 & 0.85 & 76.8 & 17.5 & 83 & 4.93 & 45.3 & 3.86 & 16.05 & 0.23 & 2.6 & 0.82 & 0.068 & 1.50 & 38.1 & 65.7 & \begin{tabular}{|l|} 
\\
\end{tabular} & 689 \\
\hline & & & & & & 17.9 & 810 & & & & & & & & & & & & & 2.0 & 0.32 & & 1.45 & 33.3 & 31.5 & 0.11 & 52 \\
\hline & & & 008 & 28 & 6.53 & 19.4 & 880 & & & 10 & & & & & 5.2 & 2 & 3.29 & .30 & & 2. & 0.33 & 0.052 & 1.67 & 34.0 & 31.4 & 0.70 & 466 \\
\hline & & 7247 & 0.012 & 1.10 & 5.91 & 21.1 & 1060 & 1.43 & 0.1 & 1.36 & \begin{tabular}{|l|}
1.75 \\
\end{tabular} & 94.9 & 13.7 & 117 & 6.59 & 42.5 & 2.74 & 15.40 & 0.18 & 2.3 & 0.83 & 0.053 & 1.76 & 50.6 & 31.6 & \begin{tabular}{|l|l|}
0.63 \\
\end{tabular} & 417 \\
\hline & & & & & & & & & & & & & & & & & & & & & 011 & & 1.17 & & 3.1 & 1061 & 60 \\
\hline & & & 0.005 & 0.72 & 5.60 & 17.5 & 950 & & 0. & 1.37 & 1.46 & 88.0 & 13 & & 5. & 35.1 & 2.61 & 14.20 & 15 & 2.3 & 0.66 & .044 & 1.54 & 46.8 & 33.0 & 0.63 & 489 \\
\hline & & & IS & & 7.15 & 11.4 & 910 & 1. & 0. & 1.06 & 0.29 & 85 & 15.4 & 8 & 6.3 & 27. & 3.26 & 17.50 & 0 & 2.7 & 0.40 & .054 & 2.20 & 42.2 & 72.3 & \begin{tabular}{|l|l|} 
\\
\end{tabular} & 47 \\
\hline & & & & & 77 & 9. & 940 & & & & & & & & & & 3.63 & & & 2. & & & 2.01 & 46.4 & 35.2 & 0.88 & 25 \\
\hline 926 & & 7244064 & 0.016 & 0.19 & 7.16 & 8.6 & 870 & 1.69 & 0.26 & 1.08 & 0.19 & 108.0 & 17.3 & 100 & 4.0 & 29. & 3.43 & 18.55 & 0. & 3.2 & 18.30 & .059 & 2.13 & 53.6 & 42.0 & 0.81 & 884 \\
\hline & & $12 \pi 000$ & 0.011 & \begin{tabular}{|l|}
0.16 \\
\end{tabular} & & 7.6 & 810 & & 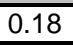 & & & 18.1 & 15.3 & 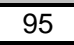 & 0.17 & & 3.28 & & & & 0.33 & & 1.64[ & 39.9 & & 0.91 & \\
\hline
\end{tabular}


Table 1. Location and New Trace Element Geochemical Results for 902 Stream-Sediment Samples collected in the Livengood Area, Livengood Quadrangle, Alaska IS = Insufficient Sample for Analysis

\begin{tabular}{|c|c|c|c|c|c|c|c|c|c|c|c|c|c|c|c|c|c|c|c|c|c|c|c|c|c|c|}
\hline SAMPLE & $\begin{array}{l}\text { Mo } \\
\text { ppm }\end{array}$ & $\begin{array}{c}\mathrm{Na} \\
\%\end{array}$ & $\begin{array}{l}\mathrm{Nb} \\
\mathrm{ppm}\end{array}$ & $\begin{array}{c}\mathrm{Ni} \\
\mathrm{ppm}\end{array}$ & $\begin{array}{c}\mathrm{P} \\
\mathrm{ppm}\end{array}$ & $\begin{array}{l}\mathrm{Pb} \\
\mathrm{ppm}\end{array}$ & $\begin{array}{l}\mathrm{Rb} \\
\mathrm{ppm}\end{array}$ & $\begin{array}{c}\mathrm{Re} \\
\mathrm{ppm}\end{array}$ & $\begin{array}{l}\mathrm{S} \\
\%\end{array}$ & $\begin{array}{c}\mathrm{Sb} \\
\mathrm{ppm}\end{array}$ & \begin{tabular}{|c|} 
Se \\
ppm
\end{tabular} & $\begin{array}{l}\text { Sn* } \\
\text { ppm }\end{array}$ & $\begin{array}{c}\mathrm{Sr} \\
\mathrm{ppm}\end{array}$ & $\begin{array}{l}\mathrm{Ta}^{\star} \\
\mathrm{ppm}\end{array}$ & $\begin{array}{c}\mathrm{Te} \\
\mathrm{ppm}\end{array}$ & \begin{tabular}{|c|} 
Th \\
ppm
\end{tabular} & $\begin{array}{l}\mathrm{Ti}^{\star} \\
\%\end{array}$ & $\begin{array}{c}\mathrm{TI} \\
\mathrm{ppm}\end{array}$ & \begin{tabular}{|c|} 
\\
ppm
\end{tabular} & $\begin{array}{c}\mathrm{V} \\
\mathrm{ppm}\end{array}$ & $\begin{array}{l}W^{*} \\
\text { ppm }\end{array}$ & $\begin{array}{c}\mathrm{Y} \\
\mathrm{ppm}\end{array}$ & $\begin{array}{c}\mathrm{Zn} \\
\mathrm{ppm}\end{array}$ & $\begin{array}{l}Z r^{\star} \\
\mathrm{ppm}\end{array}$ & $\begin{array}{c}\text { Pulp Wt } \\
\text { grams }\end{array}$ & $\begin{array}{c}\text { Lab } \\
\text { Report }\end{array}$ \\
\hline 767 & 0.85 & 1.32 & 10.6 & 33.8 & 740 & 59.7 & 67.9 & $<0.002$ & 0.03 & 1.89 & $<1$ & 2.70 & 199.5 & 0.78 & $<0.05$ & \begin{tabular}{|l|}
8.2 \\
\end{tabular} & 0.474 & 0.42 & 2.1 & 127 & 1.1 & 16.3 & 158 & 69.8 & 8 & FA04030730 \\
\hline 799 & $\overline{84}$ & 68 & 11.1 & 31.9 & 680 & 18.5 & 56.3 & $<0.002$ & 0.03 & 1.53 & 1 & 2.10 & 21.5 & 0.76 & 0.09 & 7.4 & 0.454 & 0.39 & 3.0 & 167 & 12 & 15.8 & 91 & 80.8 & 17 & FA04030730 \\
\hline 802 & 1.66 & 0.90 & 9.8 & 22.0 & 690 & 13.5 & 55.2 & $<0.002$ & 0.03 & 1.35 & $<1$ & 1.90 & 152.0 & 0.74 & 0.07 & 8.2 & 0.423 & 0.38 & 2.8 & 135 & 1.1 & 14.4 & 68 & 77.2 & 20 & FA04030730 \\
\hline & 2.18 & 96 & 11.0 & 26.6 & 830 & 17.2 & 67.7 & $<0.002$ & 0.04 & 1.28 & 1 & 1.80 & 160.0 & 0.78 & 0.06 & 8.6 & 0.441 & 0.43 & 3.0 & 141 & 1.5 & 16.0 & 83 & 79.4 & 27 & FA04030730 \\
\hline 804 & 1.54 & 1.05 & 11.5 & 26.7 & 710 & 15.8 & 71.0 & $<0.002$ & 0.03 & 1.11 & $<1$ & 1.80 & 183.5 & 0.89 & 0.05 & 9.8 & 0.439 & 0.42 & 2.7 & 128 & 1.6 & \begin{tabular}{|l|}
19.8 \\
\end{tabular} & 83 & 79.6 & 21 & FA04030730 \\
\hline 805 & 1.26 & 1.21 & 11.0 & 26.4 & 790 & 13.6 & 59.3 & $<0.002$ & 0.03 & 1.12 & 1 & 1.60 & 209.0 & 0.81 & $<0.05$ & 10.0 & 0.439 & 0.38 & 2.7 & 121 & 1.3 & 16.8 & 79 & 75.3 & 3 & FA04030730 \\
\hline 806 & 1.52 & .06 & 10.1 & 25.2 & 850 & & 0.2 & $<0.002$ & 0.04 & 1.21 & & 1.60 & 185.0 & \begin{tabular}{|l|l|}
0.77 \\
\end{tabular} & \begin{tabular}{l|l|}
0.05 \\
\end{tabular} & 8.3 & 0.419 & 0.39 & 2.6 & 128 & & 17.2 & 89 & 71.7 & & \\
\hline 807 & 1.54 & 1.18 & 11.2 & 29.0 & 620 & 15.0 & 60.5 & $<0.002$ & 0.03 & 1.33 & $\overline{1}$ & 1.90 & 206.0 & 0.81 & 0.05 & 9.3 & 0.442 & 0.44 & 2.9 & 133 & 1.6 & 19.4 & 89 & 79.4 & 22 & FA04030730 \\
\hline 812 & 1.30 & 1.35 & 12.0 & 32.8 & 840 & 14.8 & 65.7 & $<0.002$ & 0.02 & 1.42 & 1 & 1.90 & 234.0 & 0.87 & $<0.05$ & 9.6 & 0.470 & 0.43 & 2.5 & 139 & 1.2 & 19.0 & 89 & 81.0 & 22 & FA04030730 \\
\hline 813 & 0.89 & 1.39 & 12.2 & 28.7 & 880 & 12.4 & 59.8 & $<0.002$ & 0.02 & 1.13 & 1 & 1.80 & 241.0 & \begin{tabular}{|l|}
0.92 \\
\end{tabular} & $<0.05$ & 9.8 & 0.483 & 0.35 & 2.5 & 123 & 13 & 18.0 & 82 & 82.0 & & \\
\hline 814 & 0.81 & 1.38 & 11.1 & 27.7 & 810 & 11.4 & 58.6 & $<0.002$ & 0.02 & 1.05 & $<1$ & 1.60 & 234.0 & 0.83 & $<0.05$ & 8.6 & 0.431 & 0.36 & 2.3 & 115 & 1.1 & 16.8 & 79 & 75.6 & 3 & FA04030730 \\
\hline 815 & 0.85 & 1.40 & 11.2 & 28.6 & 830 & 11.8 & 58.5 & $<0.002$ & 0.02 & 1.13 & $<1$ & 1.60 & 238.0 & 0.83 & $<0.05$ & 8.6 & 0.421 & 0.34 & 2.2 & 115 & 1.2 & 16.6 & 82 & 73.7 & $\angle$ & FA04030730 \\
\hline 16 & 1.08 & 1.76 & \begin{tabular}{|l|}
15.2 \\
\end{tabular} & 34.8 & 1060 & 15.3 & 73.7 & $<0.002$ & 0.03 & 1.47 & 1 & 2.00 & 301.0 & 1.10 & $<0.05$ & 11.8 & 0.565 & 0.43 & 3.2 & 144 & 1.7 & 22.9 & 96 & 102.0 & & FA04030730 \\
\hline 822 & 1.90 & 1.02 & 10.2 & 27.3 & 1040 & 12.2 & 54.8 & $<0.002$ & 0.04 & 2.06 & 1 & 1.60 & 208.0 & 0.79 & 0.05 & 8.4 & 0.412 & 0.71 & 2.8 & 161 & 1.1 & 17.1 & 82 & 74.3 & 2 & FA04030730 \\
\hline 2 & 2.04 & 0.98 & 9.8 & 29.6 & 1280 & 13.3 & 54.9 & $<0.002$ & 0.04 & 2.18 & & 1.60 & 208.0 & 0.76 & 0.06 & 7.7 & 0.421 & 0.69 & 3.0 & 170 & 1.4 & \begin{tabular}{|l|}
17.4 \\
\end{tabular} & 83 & 73.4 & & FA04030730 \\
\hline & 1.98 & 0.53 & 5.7 & 56.1 & 1240 & 36.6 & 44.8 & $<0.002$ & 0.25 & 1.74 & 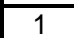 & 1.50 & 143.5 & 0.42 & 0.05 & 4.9 & 0.263 & 3.02 & 4.4 & 102 & & 20.2 & 1135 & 48 & & 0730 \\
\hline 825 & 2.26 & \begin{tabular}{|l|l|}
0.47 \\
\end{tabular} & 5.4 & 52.9 & 1200 & 35.7 & 43.3 & $<0.002$ & 0.26 & 1.65 & 1 & 1.60 & 133.5 & 0.39 & $<0.05$ & 4.6 & 0.234 & 3.00 & 4.9 & 94 & 0.6 & 20.4 & 1110 & 47.6 & 1 & FA04030730 \\
\hline 8 & 1.24 & 1.11 & 10.9 & 52.1 & 940 & 12.9 & 68.8 & $<0.002$ & 0.04 & 1.97 & & 1.50 & 197.5 & \begin{tabular}{|l|}
0.77 \\
\end{tabular} & $<0.05$ & 8.4 & 0.443 & 0.47 & 2.7 & 136 & 1. & 17.6 & 104 & 79.5 & & FA04030730 \\
\hline$\overline{66}$ & $\overline{1.72}$ & 1.10 & 11.0 & 21.3 & 670 & 13.8 & 67.3 & $<0.002$ & 0.03 & 1.63 & 1 & 1.80 & 180.0 & 0.86 & $<0.05$ & 9.3 & 0.433 & 0.42 & 2.7 & 127 & 1. & 14.4 & 65 & 73 & & FA04030730 \\
\hline 867 & 1.54 & 1.12 & $\overline{10.2}$ & 22.1 & 750 & 13.4 & 59.7 & $<0.002$ & 0.03 & 1.53 & & 1.60 & 188.5 & 0.78 & 0.05 & 7.9 & 0.399 & 0.36 & 2.4 & 119 & 1.2 & 15.0 & $\overline{6}$ & 69.0 & & FA04030730 \\
\hline 8 & 1.52 & 1.12 & 10.6 & 22.0 & 790 & 13.2 & 63.8 & $<0.002$ & 0.04 & 1.42 & & 1.80 & 189.0 & 0.74 & $<0.05$ & 7.9 & 0.402 & 0.38 & 2.4 & 121 & & 15.2 & & 70 & & 0730 \\
\hline 902 & 0.97 & \begin{tabular}{|l|}
0.77 \\
\end{tabular} & 9.6 & 24.1 & 690 & 14.9 & 62.0 & $<0.002$ & 0.04 & 0.89 & 1 & 1.80 & 129.0 & 0.71 & $<0.05$ & 7.4 & 0.407 & 0.45 & 1.9 & 114 & 1.1 & 11.4 & 83 & 61.9 & 2 & FA04030730 \\
\hline 904 & 0.98 & 0.79 & 12.6 & 44.3 & 760 & 13.8 & 69.4 & 0.002 & 0.07 & 1.60 & & 1.80 & 149.5 & \begin{tabular}{|l|}
0.79 \\
\end{tabular} & $<0.05$ & 7.8 & 0.416 & 0.44 & 2.2 & 154 & 1. & 21.1 & 126 & 62.3 & 2 & FA04030730 \\
\hline & 0.79 & 0.91 & 12.0 & 33.6 & 730 & 13.2 & 69.7 & $<0.002$ & 0.11 & 1.70 & & 2.30 & 169.0 & 0.82 & $<0.05$ & 8.1 & 0.428 & 0.44 & 2.1 & 130 & & 16.2 & 99 & 70. & & FA040 \\
\hline 907 & 1.62 & 0.75 & 16.0 & 35.3 & 840 & 15.1 & 74.6 & 0.002 & 0.04 & 3.02 & & 2.40 & 169.5 & 1.05 & 0.05 & 9.2 & 0.575 & 0.48 & 2.0 & 154 & 12 & 16.0 & 98 & 73.9 & & FA04030730 \\
\hline 908 & 0.84 & 0.99 & 11.2 & 25.7 & 540 & 11.2 & 48.6 & $<0.002$ & 0.02 & 1.08 & & 1.80 & 154.0 & 0.78 & $<0.05$ & 7.9 & 0.638 & 0.30 & 1.7 & 138 & & 13.0 & 6 & 69.9 & & FA04030730 \\
\hline & $\overline{92}$ & 0.94 & 9.9 & 28.6 & 620 & 12.2 & 52.2 & $<0.002$ & 0.03 & 1.17 & & 1.70 & 149.5 & 0.70 & $<0.05$ & 7.2 & 0.544 & 0.32 & 1.8 & 141 & & 13.2 & 66 & 64 & 4 & \\
\hline 9 & 0.77 & 0.85 & 9.7 & 31.9 & 640 & 13.0 & 56.9 & $<0.002$ & 0.06 & 1.02 & & 1.80 & 142.5 & 0.69 & $<0.05$ & 7.7 & 0.478 & 0.35 & 1.8 & 132 & & \begin{tabular}{|l|l}
14.7 \\
\end{tabular} & 104 & 66.1 & & FA04030730 \\
\hline 911 & 1.19 & 0.80 & 11.4 & 39.8 & 650 & 13.7 & 61.1 & 0.002 & 0.07 & 0.97 & & 1.80 & 148.5 & 0.78 & $<0.05$ & 8.2 & 0.465 & 0.44 & 1.9 & 131 & & 15.4 & 111 & 68. & & FA04030730 \\
\hline & 2.07 & \begin{tabular}{|l|}
0.46 \\
\end{tabular} & 22.4 & 46.6 & 1430 & 16.4 & 83.1 & 0.003 & 0.06 & 2.59 & & 1.90 & 143.5 & 1.26 & \begin{tabular}{|l|}
0.08 \\
\end{tabular} & 9.5 & 0.407 & 0.52 & 1.7 & 37 & & 14.6 & 154 & & & FA04030730 \\
\hline 913 & 2.03 & \begin{tabular}{|l|}
0.45 \\
\end{tabular} & 23.1 & 46.7 & 1440 & 16.3 & 84.7 & 0.003 & 0.06 & 2.64 & & 1.90 & 149.0 & 1.28 & 0.07 & 9.5 & 0.410 & 0.46 & 1.7 & 135 & 1. & 14.8 & 154 & 64.9 & 2 & FA04030730 \\
\hline 914 & 1.78 & \begin{tabular}{|l|}
0.45 \\
\end{tabular} & 17.6 & 39.7 & 1020 & 13.4 & 65.3 & $<0.002$ & 0.05 & 2.32 & & 1.80 & 112.5 & 1.04 & 0.06 & 7.8 & 0.427 & 0.39 & 1.6 & 130 & 1.4 & 13.0 & 146 & 67.6 & & FA04030731 \\
\hline & 1.67 & \begin{tabular}{|l|}
0.93 \\
\end{tabular} & \begin{tabular}{|l|}
15.4 \\
\end{tabular} & 30.5 & 900 & 12.5 & 61.6 & $<0.002$ & 0.04 & 2.55 & & 2.10 & 192.0 & 0.97 & $<0.05$ & 11.1 & 0.585 & 0.41 & 2.3 & 149 & & 16.6 & 97 & & & FA04030731 \\
\hline 916 & 1.92 & 0.53 & 22.4 & 49.5 & 1140 & 16.3 & 81.2 & 0.003 & 0.08 & 2.52 & 3 & 2.00 & 137.5 & 1.20 & 0.08 & 10.0 & 0.429 & 0.50 & 2.4 & 138 & & \begin{tabular}{|l|}
17.1 \\
\end{tabular} & 148 & 89.6 & & FA04030731 \\
\hline 917 & 1.60 & 0.69 & \begin{tabular}{|l|}
10.4 \\
\end{tabular} & 34.4 & 1200 & 13.3 & 76.0 & $<0.002$ & 0.08 & 6.79 & & 2.00 & 127.0 & 0.68 & 0.05 & \begin{tabular}{|l|}
9.0 \\
\end{tabular} & 0.467 & 0.54 & 2.3 & 135 & 1.2 & 18.1 & 96 & 66.5 & 2 & FA04030731 \\
\hline & 1.63 & \begin{tabular}{|l|}
0.67 \\
\end{tabular} & \begin{tabular}{|l|l|}
10.7 \\
\end{tabular} & 34.4 & 1440 & & 79.2 & $<0.002$ & 0.08 & 6.73 & & 2.10 & 123.0 & \begin{tabular}{|l|}
0.74 \\
\end{tabular} & $<0.05$ & 9.2 & 0.489 & 0.57 & 2.3 & 137 & & & 104 & 68.9 & & FA04030731 \\
\hline 919 & 5.41 & \begin{tabular}{|l|}
0.58 \\
\end{tabular} & 9.9 & 46.1 & 2520 & 13.3 & 83.7 & 0.006 & 0.06 & 3.54 & 5 & 2.00 & 151.5 & 0.70 & 0.06 & 9.5 & 0.386 & 0.83 & 3.8 & 204 & 1.2 & 29.3 & 188 & 78.5 & 20 & FA04030731 \\
\hline 9 & 2.21 & 0.67 & 9.4 & 25.5 & 870 & 10.5 & 66.4 & 0.002 & 0.08 & 2.60 & & 1.70 & 136.0 & 0.59 & 0.05 & 7.9 & 0.355 & 0.53 & 2.4 & 118 & & \begin{tabular}{|l|}
15.3 \\
\end{tabular} & 102 & 64.5 & 1 & FA04030731 \\
\hline & 4.07 & 0.62 & 9.9 & 37.5 & 2000 & 12.8 & 82.5 & 0.004 & 0.06 & 3.25 & 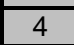 & 1.90 & 152.0 & 0.66 & 0.07 & 9.4 & 0.441 & 0.79 & 3.8 & 191 & & \begin{tabular}{|l|}
25.4 \\
\end{tabular} & 174 & 77.7 & & FA04030731 \\
\hline 923 & 1.03 & \begin{tabular}{|l|}
0.64 \\
\end{tabular} & \begin{tabular}{|l|}
10.7 \\
\end{tabular} & 35.3 & 690 & 21.1 & 112.5 & $<0.002$ & 0.06 & 1.33 & 2 & 2.30 & 148.0 & 0.80 & $<0.05$ & 14.8 & 0.414 & 0.62 & 2.8 & 104 & 1.6 & \begin{tabular}{|l|}
17.8 \\
\end{tabular} & 88 & 89.1 & 20 & FA04030731 \\
\hline & 0.98 & \begin{tabular}{|l|}
0.84 \\
\end{tabular} & 12.6 & 36.2 & 620 & 18.4 & 98.5 & $<0.002$ & 0.05 & 1.06 & & 2.50 & 180.5 & 0.90 & $<0.05$ & 13.6 & 0.468 & 0.55 & 2.6 & 115 & & -1 & 106 & $78 . \varepsilon$ & 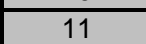 & FA04030731 \\
\hline 926 & 0.74 & 0.81 & \begin{tabular}{|l|}
13.8 \\
\end{tabular} & 38.1 & 570 & 16.1 & 100.0 & $<0.002$ & 0.06 & 0.89 & 1 & 2.60 & 187.0 & 0.95 & $<0.05$ & 16.8 & 0.492 & 0.55 & 3.0 & 104 & $1 . !$ & 20.2 & 128 & 112.5 & 15 & FA0403 \\
\hline 927 & 0.91 & 1.08 & 12.1 & 35.6 & 600 & 14.5 & 76.7 & $<0.002$ & 0.05 & 1.30 & & 2.00 & 206.0 & \begin{tabular}{|l|}
0.81 \\
\end{tabular} & $<0.05$ & \begin{tabular}{|l|}
11.5 \\
\end{tabular} & \begin{tabular}{|l|}
0.471 \\
\end{tabular} & \begin{tabular}{|l|}
0.44 \\
\end{tabular} & \begin{tabular}{|l|}
2.7 \\
\end{tabular} & 107 & 1.4 & \begin{tabular}{|l|}
17.2 \\
\end{tabular} & \begin{tabular}{|l|}
107 \\
\end{tabular} & 74.8 & 25 & FA0403073. \\
\hline
\end{tabular}


Table 1. Location and New Trace Element Geochemical Results for 902 Stream-Sediment Samples collected in the Livengood Area, Livengood Quadrangle, Alaska IS = Insufficient Sample for Analysis

\begin{tabular}{|c|c|c|c|c|c|c|c|c|c|c|c|c|c|c|c|c|c|c|c|c|c|c|c|c|c|c|c|}
\hline MPLE & TM E & TM N & $\begin{array}{c}\mathrm{Au} \\
\mathrm{ppm}\end{array}$ & $\begin{array}{l}\mathrm{Ag} \\
\mathrm{ppm}\end{array}$ & $\begin{array}{l}\text { Al } \\
\%\end{array}$ & $\begin{array}{l}\text { As } \\
\text { ppm }\end{array}$ & $\begin{array}{l}\mathrm{Ba}^{*} \\
\mathrm{ppm}\end{array}$ & $\begin{array}{c}\mathrm{Be} \\
\mathrm{ppm} \\
\end{array}$ & $\begin{array}{c}\mathrm{Bi} \\
\mathrm{ppm}\end{array}$ & $\begin{array}{c}\mathrm{Ca} \\
\% \\
\end{array}$ & $\begin{array}{c}\mathrm{Cd} \\
\mathrm{ppm}\end{array}$ & $\begin{array}{c}\mathrm{Ce} \\
\mathrm{ppm}\end{array}$ & $\begin{array}{l}\mathrm{Co} \\
\mathrm{ppm}\end{array}$ & $\begin{array}{l}\mathrm{Cr}^{*} \\
\mathrm{ppm}\end{array}$ & $\begin{array}{l}\text { CS } \\
\text { ppm }\end{array}$ & $\begin{array}{r}\mathrm{Cu} \\
\mathrm{ppm}\end{array}$ & $\begin{array}{c}\mathrm{Fe} \\
\% \\
\end{array}$ & $\begin{array}{c}\mathrm{Ga} \\
\mathrm{ppm}\end{array}$ & $\begin{array}{l}\mathrm{Ge} \\
\mathrm{ppm}\end{array}$ & $\begin{array}{c}\mathrm{Hf} \\
\mathrm{ppm} \\
\end{array}$ & $\begin{array}{l}\mathrm{Hg} \\
\mathrm{ppm}\end{array}$ & $\begin{array}{c}\text { In } \\
\text { ppm }\end{array}$ & $\begin{array}{l}\mathrm{K} \\
\% \\
\end{array}$ & $\begin{array}{r}\mathrm{La} \\
\mathrm{ppm}\end{array}$ & $\begin{array}{c}\mathrm{Li} \\
\mathrm{ppm} \\
\end{array}$ & $\begin{array}{c}\mathrm{Mg} \\
\% \\
\end{array}$ & $\begin{array}{l}\mathrm{Mn} \\
\mathrm{ppm}\end{array}$ \\
\hline 928 & 9199 & 44412 & $<0.005$ & \begin{tabular}{|l|l|}
0.17 \\
\end{tabular} & \begin{tabular}{|l|l|}
6.54 \\
\end{tabular} & 8.0 & 770 & 1.36 & 0.19 & & \begin{tabular}{|l}
0.23 \\
\end{tabular} & 82.8 & 15.9 & 97 & & 23.2 & 3.30 & 15.80 & 0.14 & \begin{tabular}{|l|}
2.5 \\
\end{tabular} & 0.26 & 0.052 & 1.61 & 41.8 & 36.5 & 0.90 & 723 \\
\hline 29 & & & 006 & & \begin{tabular}{|l|l}
7.05 \\
\end{tabular} & 8.1 & 890 & & & 32 & \begin{tabular}{|l|}
0.19 \\
\end{tabular} & 89. & 17. & & & 5.9 & 3.47 & 17.65 & 0.15 & 2.8 & 0.89 & 0.054 & \begin{tabular}{|l|}
2.04 \\
\end{tabular} & 45.0 & 9.0 & \begin{tabular}{|l|l}
0.90 \\
\end{tabular} & 50 \\
\hline & & & .005 & 50 & 7.10 & 2.8 & 1340 & 60 & 0.23 & 82 & 58 & 0.0 & 12.7 & 04 & .7 & 3.7 & 3.02 & 8.15 & .15 & 3.0 & 0.41 & 054 & \begin{tabular}{|l|}
2.12 \\
\end{tabular} & 47.3 & 5.8 & 0.65 & 63 \\
\hline & & & & 41 & \begin{tabular}{|l|l|}
7.18 \\
\end{tabular} & 1.2 & 660 & & & & & 40 & 13.8 & & & $\overline{3.2}$ & 2.99 & 8.05 & 0.16 & & 0.56 & & \begin{tabular}{|l|}
2.21 \\
\end{tabular} & 4 & 0.7 & 0.72 & 25 \\
\hline & & & 0.017 & 36 & \begin{tabular}{|l|l|}
6.97 \\
\end{tabular} & 10.5 & 020 & .62 & 0.23 & .06 & .54 & 87.0 & 14.4 & 99 & 32 & 30.3 & 2.94 & 18.70 & 0.16 & 2.8 & 1.01 & 0.053 & 2.21 & 44.2 & 2.2 & 0.73 & 787 \\
\hline & & & .005 & 29 & 7.01 & 8.8 & 1020 & $\overline{49}$ & 0.21 & 1.24 & \begin{tabular}{|l|l|}
0.47 \\
\end{tabular} & 76.9 & 17.1 & 101 & 4.63 & $\overline{8.8}$ & 3.29 & 17.30 & 0.17 & 2.4 & 0.54 & .053 & 1.93 & 38.7 & 53.9 & 0.89 & 1795 \\
\hline & & & .026 & & 6.19 & & 900 & & & & \begin{tabular}{|l|l|}
0.46 \\
\end{tabular} & 79.3 & 16.8 & & 15 & & 289 & 16.90 & 0.14 & 2.4 & & & & 39.1 & & 0.79 & \\
\hline & & & 0.012 & 16 & 6.00 & 7.2 & 710 & $\overline{09}$ & 0.17 & 0.65 & 15 & 70.3 & 8.9 & 80 & 3.24 & 12.9 & 2.39 & 15.30 & 0.13 & 2.4 & 0.13 & 0.037 & 1.59 & 34.5 & 1.7 & 0.65 & 337 \\
\hline & & & .005 & 21 & 18 & & 720 & 1.23 & 0.21 & $\overline{93}$ & 0.35 & 82.4 & 20.3 & & 2.89 & 7.5 & 2.93 & 15.65 & 0.16 & 2.5 & 0.17 & 0.045 & 1.50 & 39.9 & 34.8 & 0.76 & 325 \\
\hline & & & .005 & 12 & 31 & & 740 & 1.30 & 0.18 & 84 & \begin{tabular}{|l}
0.23 \\
\end{tabular} & 82.1 & 21.0 & & 2.99 & 55 & 2.84 & 15.90 & 0.14 & 2.4 & 0.29 & 0.045 & \begin{tabular}{|l|}
1.57 \\
\end{tabular} & 39.2 & 2.4 & 0.75 & 332 \\
\hline & & & 0.010 & 0.15 & 6.25 & 6. & 760 & 1.30 & 0.16 & 0.88 & \begin{tabular}{|l|l|} 
\\
\end{tabular} & 86.8 & 20. & & 2.91 & 15.8 & 2.86 & 15.10 & 0.14 & 3.3 & 0.13 & 0.048 & \begin{tabular}{|l|l}
1.67 \\
\end{tabular} & 44.8 & \begin{tabular}{|l|}
34.6 \\
\end{tabular} & \begin{tabular}{|l}
0.77 \\
\end{tabular} & 916 \\
\hline & & & .005 & 14 & .58 & & 820 & 4 & & .97 & \begin{tabular}{|l}
0.53 \\
\end{tabular} & & 28 & & .1 & 9. & 3.11 & 16.35 & 0.16 & 2.7 & 0.20 & 0.048 & \begin{tabular}{|l|}
1.71 \\
\end{tabular} & 44.4 & 36.9 & \begin{tabular}{|l}
0.77 \\
\end{tabular} & $3 / 6$ \\
\hline & & & .005 & 21 & & & 850 & 51 & & .94 & 45 & 2.7 & 22. & & 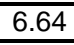 & 1 & 3.30 & 17.25 & 0.15 & 2. & 0.27 & .052 & \begin{tabular}{|l|l}
1.82 \\
\end{tabular} & $\overline{0}$ & 7.8 & 0.80 & 610 \\
\hline & & & 0.005 & 0.11 & 6.89 & 6.8 & 780 & 1.52 & 0.16 & 0.84 & \begin{tabular}{|l|l|}
0.28 \\
\end{tabular} & 82.8 & 16.5 & 8 & 6.93 & 15.6 & 3.05 & 16.60 & 0.16 & 2.7 & 0.21 & .046 & 1.74 & 41.8 & 53.2 & 0.73 & 927 \\
\hline & & & 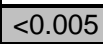 & & & & 820 & & & & & & 11.3 & & & & 2.72 & .35 & 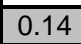 & 1.9 & & .040 & \begin{tabular}{|l|}
1.25 \\
\end{tabular} & & 20.0 & 0.86 & 549 \\
\hline & & & .005 & 16 & 5.35 & 4.5 & 1320 & & 0.1 & 1.12 & 24 & 64. & 8.3 & 7 & 2.9 & $\overline{20.8}$ & 2.17 & 2.60 & 0.10 & 2.2 & 0.23 & 039 & \begin{tabular}{|l|}
1.21 \\
\end{tabular} & 30.9 & 0.1 & & 322 \\
\hline & & & .005 & 14 & 5.31 & & 970 & .14 & 0.11 & .46 & 24 & 6.7 & 10. & & 2.2 & 18. & 2.41 & 2.30 & 0.12 & 2. & 0.16 & .040 & 1.28 & 33.2 & 0.2 & 0.81 & 408 \\
\hline & & & .005 & $\overline{15}$ & & & 44 & & & 71 & & & & & & & & 2.35 & & & & & 1.. & & 8.8 & $\overline{0.9}$ & 78 \\
\hline & & & 0.005 & 0.23 & 5.46 & 9. & 1000 & 1.21 & 0.16 & 1.07 & 0.38 & 68.5 & 20. & & 3.26 & 37.0 & 3.05 & 13.70 & 0.14 & 2.4 & 0.73 & $\overline{046}$ & \begin{tabular}{|l|}
1.27 \\
\end{tabular} & 33.3 & 18.9 & 0.73 & 1215 \\
\hline & & & 005 & & 5 & & & & & 1.29 & & & & & 2.8 & 34. & 3.29 & 13.25 & 0.14 & 2.2 & 0.34 & 042 & 1.26 & 33.0 & \begin{tabular}{|l|l|}
18.6 \\
\end{tabular} & 0.84 & 1040 \\
\hline & & & 0.005 & 27 & 5.10 & 9 & 1100 & & & 44 & & & 20. & & $2.6 s$ & 45. & 3.18 & 1.90 & 0.13 & & 0.36 & 044 & & 33.3 & 9.8 & & 1465 \\
\hline & & & $<0.005$ & 0.22 & 5.27 & 8. & 1020 & 1.14 & 0.15 & 1.36 & 1.10 & 85.8 & 23. & & 2.84 & 38.0 & 2.93 & 12.35 & 0.14 & 2.1 & 0.60 & .042 & \begin{tabular}{|l|l|}
1.14 \\
\end{tabular} & 38.3 & 20.9 & 0.77 & 1385 \\
\hline & & & .005 & 18 & \begin{tabular}{|l|l|}
5.80 \\
\end{tabular} & & 940 & & & 31 & 41 & & 16. & & 3.1 & 27. & 3.19 & 4.25 & 0.15 & 2.4 & 0.30 & .048 & 1.26 & 36.3 & 32.5 & 0.85 & 328 \\
\hline & & & .005 & 16 & 5.98 & 7. & 870 & & & 62 & 24 & 102. & 12. & & 2.7 & & & & & 3.1 & & & \begin{tabular}{|l}
1.28 \\
\end{tabular} & 52.7 & \begin{tabular}{|l|}
25.1 \\
\end{tabular} & 0.94 & 513 \\
\hline & & & .008 & $\overline{14}$ & 5.86 & & 880 & & 0.1 & 62 & 37 & 86.3 & 19. & & 2.7 & & 3.24 & 3.55 & 0.1 & 2. & 0.2 & 0.045 & 1.25 & 43.6 & 25.7 & 0.91 & 1120 \\
\hline & & & 005 & 15 & & & 830 & & & 52 & & & & & .5 & & 2.79 & 2.80 & & & & 040 & 1.23 & 3.9 & 3.6 & 0.85 & 683 \\
\hline & & & .005 & 16 & 5.75 & 8. & 850 & & & 1.54 & \begin{tabular}{|l|l|}
0.38 \\
\end{tabular} & & 17. & & 2.69 & & 2.88 & 3.40 & & 2.3 & 0.29 & & \begin{tabular}{|l|}
1.30 \\
\end{tabular} & 36.2 & 4.8 & 0.85 & 1070 \\
\hline & & & .005 & & & & 670 & & & 24 & & & 9. & & 3.2 & & 2.83 & 3.90 & 0 & 2. & 0.05 & 0 & 1.36 & 47.7 & 26.8 & 0.79 & 428 \\
\hline & & & & & & & 800 & & & & & & & & & & & & & & & & & & 0.3 & $0 . \varepsilon$ & 13 \\
\hline & & & & & & & & & & & & & & & & & & & & & & & & & & & \\
\hline & & & .005 & 15 & 5.74 & 8.0 & 800 & & & 1.37 & & 74.6 & & & 2.73 & & 2.80 & 3.80 & 13 & 2.3 & 0.21 & .044 & 1.30 & 39.0 & 3.7 & 0.83 & 622 \\
\hline & & & & & & 8 & 780 & & & 42 & & & & & & & & & & 2.3 & & & 1.26 & 38 & 3 & 0.19 & 776 \\
\hline & & & .005 & & & & 820 & & & & & & & & & & 2.71 & & & $\sqrt{22}$ & $\overline{0.2}$ & & & 34 & & $\overline{0.81}$ & 1045 \\
\hline & & & 0.006 & 0.12 & 5.62 & 8.0 & 810 & & 0. & 1.50 & 0.34 & 69.8 & 13 & & 2.60 & & 2.73 & 13.65 & & 2.3 & 0.16 & .039 & 1.31 & 35.5 & 24.5 & 0.83 & 745 \\
\hline & & & & & & 8.8 & 800 & & & & & & & & & & & & & & & & 1.36 & & 3.8 & 0.85 & 06 \\
\hline & & & 007 & & 5. & 9 & 830 & & & & & & & & 2. & & 2.84 & 3.50 & & 2. & 0.22 & $\overline{41}$ & 1.32 & 34.8 & 23. & 0.85 & 734 \\
\hline & & $\overline{9}$ & 0.014 & 0.13 & 5.61 & 7.5 & 790 & 1. & 0. & 1.72 & \begin{tabular}{|l|l|}
0.29 \\
\end{tabular} & 82.2 & 13.9 & 100 & 2.3 & & 2.81 & 13.45 & & 2.9 & 0.45 & 04 & 1.28 & 43.8 & 23.5 & 0.88 & 780 \\
\hline & & & & & & & & & & & & & & & & & & & & & & & & & 3.2 & 0.98 & 27 \\
\hline 1013 & & & 0.007 & 0.33 & \begin{tabular}{|l|}
5.91 \\
\end{tabular} & & 670 & & 0. & 1.73 & & & 15.9 & & 6.27 & & 5.69 & 15.10 & 20 & 2.0 & 0.19 & 0.056 & \begin{tabular}{|l|}
1.28 \\
\end{tabular} & 67.5 & 6.7 & \begin{tabular}{|l|}
0.93 \\
\end{tabular} & 835 \\
\hline & & & $<0.005$ & \begin{tabular}{|l|}
0.29 \\
\end{tabular} & \begin{tabular}{|l|l|}
6.99 \\
\end{tabular} & 11.8 & 660 & 2. & 0. & 2.59 & 0. & 188.5 & 13.5 & 6 & 7.1 & & 3.53 & 17.95 & 0 & 2.1 & 0.14 & 0 & \begin{tabular}{|l|}
1.46 \\
\end{tabular} & 93.7 & 29.4 & \begin{tabular}{|l}
1.27 \\
\end{tabular} & 447 \\
\hline & & & & & & & & & & 2.43 & & & & & & & & & & 1. & 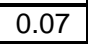 & & 1.45 & 90.5 & 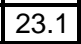 & 1.30 & $\overline{06}$ \\
\hline 101 & & 724 & 0.007 & 0.33 & 6.33 & 27.2 & 640 & 2.3 & 0.4 & 2.31 & 0.22 & $\overline{174}$ & 17.1 & 67 & 6.7 & & 3.84 & 16.30 & 0 & 2.3 & 0.15 & & 1.35 & 90.3 & 6.5 & 1.14 & 030 \\
\hline & & & $<0.005$ & & & 43.6 & 680 & & & & & & 25.9 & & $1+1$ & & 4.21 & & & & 0.36 & & & 74.1 & & 1.04 & 100 \\
\hline
\end{tabular}


Table 1. Location and New Trace Element Geochemical Results for 902 Stream-Sediment Samples collected in the Livengood Area, Livengood Quadrangle, Alaska IS = Insufficient Sample for Analysis

\begin{tabular}{|c|c|c|c|c|c|c|c|c|c|c|c|c|c|c|c|c|c|c|c|c|c|c|c|c|c|c|}
\hline SAMPLE & $\begin{array}{l}\text { Mo } \\
\text { ppm }\end{array}$ & $\begin{array}{c}\mathrm{Na} \\
\%\end{array}$ & $\begin{array}{l}\mathrm{Nb} \\
\mathrm{ppm}\end{array}$ & $\begin{array}{r}\mathrm{Ni} \\
\mathrm{ppm}\end{array}$ & $\begin{array}{c}\mathrm{P} \\
\mathrm{ppm}\end{array}$ & $\begin{array}{l}\mathrm{Pb} \\
\mathrm{ppm}\end{array}$ & $\begin{array}{l}\mathrm{Rb} \\
\mathrm{ppm}\end{array}$ & $\begin{array}{l}\mathrm{Re} \\
\mathrm{ppm}\end{array}$ & $\begin{array}{l}\mathrm{S} \\
\%\end{array}$ & $\begin{array}{l}\text { Sb } \\
\text { ppm }\end{array}$ & $\begin{array}{c}\text { Se } \\
\text { ppm }\end{array}$ & $\begin{array}{l}S^{S} n^{*} \\
\text { ppm }\end{array}$ & $\begin{array}{c}\mathrm{Sr} \\
\mathrm{ppm}\end{array}$ & $\begin{array}{l}\mathrm{Ta}^{*} \\
\mathrm{ppm}\end{array}$ & $\begin{array}{c}\text { Te } \\
\text { ppm }\end{array}$ & $\begin{array}{l}\text { Th } \\
\text { ppm }\end{array}$ & $\begin{array}{l}\mathrm{Ti}^{\star} \\
\% \\
\end{array}$ & $\begin{array}{c}\mathrm{Tl} \\
\mathrm{ppm}\end{array}$ & $\begin{array}{c}U \\
\mathrm{ppm}\end{array}$ & $\begin{array}{c}\mathrm{V} \\
\mathrm{ppm}\end{array}$ & $\begin{array}{l}W^{*} \\
\text { ppm }\end{array}$ & \begin{tabular}{|c|}
$\mathrm{Y}$ \\
$\mathrm{ppm}$
\end{tabular} & $\begin{array}{l}\mathrm{Zn} \\
\mathrm{ppm}\end{array}$ & $\begin{array}{l}\mathrm{Zr}^{*} \\
\mathrm{ppm}\end{array}$ & $\begin{array}{c}\text { Pulp Wt } \\
\text { grams }\end{array}$ & $\begin{array}{c}\text { Lab } \\
\text { Report }\end{array}$ \\
\hline 928 & 0.91 & 1.04 & 12.4 & 38.2 & 590 & 14.1 & 79.0 & \begin{tabular}{|c|}
$<0.002$ \\
\end{tabular} & 0.04 & 1.13 & 1 & 2.20 & 198.5 & 0.92 & $<0.05$ & 12.0 & 0.490 & 0.43 & 2.8 & 107 & 1.2 & 17.8 & 114 & 80.6 & 20 & FA04030731 \\
\hline 929 & 0.77 & 93 & 13.6 & 38.8 & 600 & 13.9 & 88.4 & \begin{tabular}{|c|c|}
$<002$ \\
\end{tabular} & 0.05 & 0.91 & 1 & & 202.0 & 0.90 & $<0.05$ & 13.1 & 0.538 & 0.47 & 2.4 & 116 & 1.3 & 18.0 & 114 & 88.7 & 31 & \\
\hline 930 & 3.61 & 0.63 & 11.1 & 35.7 & 890 & 15.9 & 101.5 & $<0.002$ & 0.06 & 1.84 & 3 & 2.30 & 138.5 & 0.78 & 0.06 & 13.5 & 0.429 & 0.80 & 3.6 & 139 & 1.3 & \begin{tabular}{|l|}
18.2 \\
\end{tabular} & 108 & 102.0 & $\overline{1 \varepsilon}$ & FA04030731 \\
\hline 931 & 2.08 & 0.59 & 10.9 & 37.0 & 770 & 17.3 & 109.0 & $<0.002$ & 0.06 & 4.06 & & 2.30 & 134.0 & 0.76 & 0.06 & 13.6 & 0.402 & 0.73 & 3.5 & 124 & 1.8 & 19.5 & 108 & 93.2 & $\Delta$ & FA04030731 \\
\hline 932 & .14 & 58 & 11.2 & 39.3 & 730 & 16.7 & 106.0 & $<0.002$ & 0.06 & 3.92 & 3 & 2.40 & 128.5 & 0.77 & 0.08 & 13.4 & 0.427 & 0.72 & 3.4 & 123 & 1.8 & 20.2 & 98 & 95.9 & & \\
\hline 33 & 1.47 & 0.69 & 10.7 & 44.6 & 720 & 15.6 & 94.9 & $<0.002$ & 0.07 & 2.85 & 2 & 2.00 & 155.0 & 0.72 & 0.07 & 12.5 & 0.420 & 0.66 & 3.1 & 114 & 1.6 & 18.0 & 132 & 84.5 & 20 & FA04030731 \\
\hline 9 & 1.45 & 0.63 & 10.4 & 43.9 & 650 & 15.4 & 92.4 & $<0.002$ & 0.06 & 2.75 & & 2.10 & 144.0 & 0.73 & 0.05 & 12.5 & 0.421 & 0.63 & 3.0 & 115 & 1.6 & \begin{tabular}{|l|}
18.3 \\
\end{tabular} & 106 & 81.5 & 15 & FA04030731 \\
\hline 35 & 0.77 & 0.84 & \begin{tabular}{|l|}
9.7 \\
\end{tabular} & 21.5 & 530 & 13.0 & 85.7 & $<0.002$ & 0.04 & 0.69 & 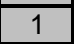 & 2.00 & 113.0 & 0.70 & 0.05 & 10.0 & 0.380 & 0.50 & 2.3 & 93 & 1.0 & 12.0 & 70 & 78.9 & & FA04030731 \\
\hline 936 & 0.71 & 0.86 & 10.3 & 30.1 & 540 & 16.6 & 78.5 & $<0.002$ & 0.04 & 0.76 & & 1.90 & 139.0 & 0.74 & $<0.05$ & 11.7 & 0.426 & 0.47 & 2.8 & 98 & 1.1 & 17.5 & 91 & 86.0 & 3 & $\overline{\text { FA040 }}$ \\
\hline 9 & .63 & 86 & 10.2 & 27.3 & 520 & 17.4 & 81.0 & $<0.002$ & 0.04 & 0.68 & & 1.90 & 133.5 & 0.73 & $<0.05$ & 11.0 & 0.414 & 0.48 & 2.6 & 98 & 1.1 & 15.9 & 84 & 83.1 & & 0731 \\
\hline 9 & .52 & 0.84 & 11.4 & 29.7 & 460 & 16.5 & 82.3 & $<0.002$ & 0.03 & 0.64 & & 1.90 & 136.5 & 0.90 & $<0.05$ & 14.2 & 0.439 & 0.46 & 3.0 & 92 & 1.2 & 19.6 & 95 & 108.5 & & 7731 \\
\hline 9 & 0.62 & 0.87 & 10.4 & 34.1 & 540 & 17.8 & 85.6 & \begin{tabular}{l|}
$<0.002$ \\
\end{tabular} & 0.04 & 0.68 & & 2.00 & 156.5 & 0.78 & $<0.05$ & 12.2 & 0.420 & 0.49 & 3.0 & 99 & 1.1 & 20.0 & 209 & 93.6 & 3 & FA04030731 \\
\hline & & 0.84 & \begin{tabular}{|l|}
9.6 \\
\end{tabular} & 36.0 & 660 & 18.5 & 90.4 & $<0.002$ & 0.05 & 0.81 & & 2.00 & 167.5 & \begin{tabular}{|l|l|}
0.68 \\
\end{tabular} & $<0.05$ & \begin{tabular}{|l|l}
11.7 \\
\end{tabular} & 0.386 & 0.58 & 3.5 & 102 & 1.2 & \begin{tabular}{|l|l|}
19.4 \\
\end{tabular} & 185 & 77.2 & & \\
\hline 9 & 0.53 & 0.86 & 10.2 & 28.8 & 490 & 15.8 & 87.8 & $<0.002$ & 0.03 & 0.63 & 1 & 2.00 & 142.0 & 0.84 & 0.05 & 12.1 & 0.398 & 0.51 & 2.9 & 94 & 1.1 & 17.6 & 86 & 87.8 & 1 & 0731 \\
\hline 975 & 0.93 & 1.15 & 8.9 & 27.9 & 730 & 10.5 & 53.1 & $<0.002$ & 0.03 & 1.00 & & 1.40 & 208.0 & 0.65 & $<0.05$ & 6.8 & 0.369 & 0.33 & 1.9 & 114 & 0.9 & 15.2 & 83 & 64.6 & 3 & FA04030731 \\
\hline & 56 & .04 & 9.7 & 22.5 & 600 & 11.4 & 59.4 & $<0.002$ & 0.03 & 0.90 & & 1.60 & 182.0 & 0.76 & $<0.05$ & 7.6 & 0.382 & 0.34 & 2.2 & 112 & 1.0 & 15.0 & 69 & 69.2 & & \\
\hline 977 & 0.69 & 1.20 & 9.7 & 24.9 & 700 & 10.4 & 54.5 & $<0.002$ & 0.02 & 0.95 & & 1.40 & 215.0 & 0.65 & $<0.05$ & 8.0 & 0.382 & 0.34 & 2.0 & 107 & 1.0 & \begin{tabular}{|l|}
15.8 \\
\end{tabular} & 70 & 72.1 & $\overline{5}$ & 0731 \\
\hline$\overline{9}$ & 0.71 & 1.25 & 10.0 & 26.5 & 760 & 11.0 & 53.5 & $<0.002$ & 0.02 & 1.07 & & 1.40 & 231.0 & 0.74 & $<0.05$ & 8.2 & 0.413 & 0.32 & 2.1 & 116 & 1. & 17.0 & 79 & 71. & & 731 \\
\hline & 3.17 & 0.87 & 11.6 & 36.0 & 1000 & 14.2 & 57.7 & $<0.002$ & 0.06 & 1.56 & 7 & 1.60 & 167.5 & \begin{tabular}{|l|}
0.81 \\
\end{tabular} & \begin{tabular}{|l|}
0.07 \\
\end{tabular} & 7.7 & 0.454 & 0.38 & 3.1 & 141 & 1. & 18.2 & 86 & 84. & & 0731 \\
\hline 991 & 1.65 & 0.95 & 11.2 & 50.4 & 940 & 11.6 & 54.7 & $<0.002$ & 0.05 & 1.42 & & 1.60 & 179.5 & 0.75 & 0.05 & 7.3 & 0.517 & 0.32 & 2.8 & 135 & 1.1 & 19.4 & 106 & 77.6 & $\overline{2}$ & 30731 \\
\hline 9 & 1.80 & 0.86 & \begin{tabular}{|l|}
9.3 \\
\end{tabular} & 46.5 & 1060 & 14.1 & 50.4 & $<0.002$ & 0.08 & 1.54 & & 1.50 & 164.5 & $\begin{array}{ll}0.61 \\
\end{array}$ & 0.06 & 6.9 & 0.395 & 0.34 & 2.7 & 131 & $\overline{0 . \varepsilon}$ & 18.8 & 108 & 69.5 & & 0731 \\
\hline & .43 & 0.90 & 9.6 & 42.3 & 940 & 13.9 & 54.4 & 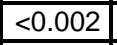 & 0.07 & 1.27 & & 1.50 & 173.0 & $\begin{array}{ll}0.68 \\
\end{array}$ & 0.05 & 7.7 & 0.402 & 0.37 & 2.7 & 12 & 1. & \begin{tabular}{|l|}
21.6 \\
\end{tabular} & 102 & 73. & & 731 \\
\hline 9 & 1.31 & 1.06 & 10.6 & 35.0 & 780 & 14.5 & 60.0 & \begin{tabular}{|l|}
0.002 \\
\end{tabular} & 0.04 & 1.12 & & 1.80 & 192.5 & $\begin{array}{ll}0.73 \\
\end{array}$ & 0.06 & 8.4 & 0.435 & 0.36 & 2.5 & 120 & 1. & \begin{tabular}{|l|}
18.1 \\
\end{tabular} & 87 & 74.7 & & 30731 \\
\hline 9 & 0.89 & 1.24 & 13.2 & 30.2 & 870 & 12.7 & 56.2 & $<0.002$ & 0.03 & 0.97 & & 1.80 & 231.0 & 0.94 & $<0.05$ & 13.0 & 0.541 & 0.35 & 3.0 & 124 & 1. & 20.7 & $r$ & 96.3 & & 731 \\
\hline & .01 & 1.18 & 11.4 & 30.5 & 860 & 12.5 & 57.3 & $<0.002$ & 0.03 & 1.00 & & 1.70 & 220.0 & \begin{tabular}{|l|}
0.79 \\
\end{tabular} & 0.05 & 10.4 & 0.467 & 0.35 & 2.4 & 121 & 1.3 & 19.4 & 85 & 83.4 & & 0731 \\
\hline 998 & 0.79 & 1.20 & 10.8 & 27.0 & 770 & 12.0 & 55.3 & $<0.002$ & 0.03 & 0.99 & & 1.60 & 220.0 & \begin{tabular}{|l|}
0.72 \\
\end{tabular} & $<0.05$ & 8.6 & 0.404 & 0.34 & 2.3 & 10 & 1.2 & 17.2 & 8 & 73.5 & & 0731 \\
\hline & 0.79 & 1.22 & 10.4 & 28.6 & 780 & 11 & 57.9 & $<0.002$ & 0.03 & 0.95 & & 1.60 & 223.0 & 0.73 & $<0.05$ & 8.5 & 0.407 & 0.37 & 2.2 & 1 & & 18.0 & & & & \\
\hline & 0.76 & 1.18 & 11.0 & 20.8 & 590 & 13.9 & 59.8 & $<0.002$ & 0.02 & 0.88 & & 1.70 & 204.0 & \begin{tabular}{|l|l|}
0.84 \\
\end{tabular} & $<0.05$ & 10.3 & 0.461 & 0.33 & 2.3 & 11 & 1 & 16.0 & 6 & 80.2 & & 0731 \\
\hline 1001 & 0.72 & 1.32 & 10.3 & 22.2 & 540 & 10.4 & 49.1 & $<0.002$ & 0.03 & 0.73 & & 1.60 & 262.0 & \begin{tabular}{|l|l|}
0.78 \\
\end{tabular} & $<0.05$ & 7.5 & 0.427 & 0.32 & 1.9 & 10 & 1. & 14.4 & 62 & 73.5 & 2 & 0731 \\
\hline & & 1.31 & \begin{tabular}{|l|}
9.8 \\
\end{tabular} & 26.4 & 650 & & 60.1 & $<0.002$ & 0.03 & 1.12 & & 1.90 & 265.0 & \begin{tabular}{|l|}
0.72 \\
\end{tabular} & $<0.05$ & 7.5 & 0.404 & 0.35 & 1.9 & 1 & & 15.1 & 83 & 72. & & \\
\hline & 0.88 & 1.25 & \begin{tabular}{|l|}
10.4 \\
\end{tabular} & 24.7 & 650 & 12.4 & 56.9 & $<<0.002$ & 0.03 & 0.91 & & 1.70 & 246.0 & \begin{tabular}{|l|}
0.72 \\
\end{tabular} & $<0.05$ & 8.5 & 0.436 & 0.35 & 2.0 & 11 & 1. & 16.0 & 7 & 73. & 2 & 7731 \\
\hline 100 & 0.76 & 1.22 & \begin{tabular}{|l|}
10.2 \\
\end{tabular} & 24.4 & 660 & 11.7 & 55.1 & $<0.002$ & 0.03 & 0.87 & & 1.60 & 223.0 & 0.70 & $<0.05$ & 9.2 & 0.417 & 0.35 & 2.3 & $\overline{11}$ & & 16.5 & & 70. & & 7731 \\
\hline & 0.92 & 1.20 & & 27.3 & 700 & & 61.5 & & 0.03 & 1.02 & & 1.70 & 219.0 & 0.68 & $<0.05$ & 8.6 & 0.399 & 0.38 & 2.2 & & & 16.8 & & & & \\
\hline 100 & 0.95 & 1.21 & 10.6 & 27.4 & 740 & 12.2 & 56.6 & $<0.002$ & 0.03 & 0.92 & & 1.60 & 221.0 & 0.71 & 0.05 & 8.3 & 0.408 & 0.36 & 2.1 & 11 & 1. & 17.3 & 82 & 68.8 & 3 & FA04030731 \\
\hline 1008 & 0.85 & 1.19 & 11.2 & 29.2 & 790 & 11.7 & 56.2 & $<0.002$ & 0.03 & 1.03 & & 1.60 & 225.0 & 0.79 & $<0.05$ & 9.0 & 0.454 & 0.34 & 2.3 & 120 & 1. & \begin{tabular}{|l|}
18.8 \\
\end{tabular} & 8 & 77.7 & 3 & 0731 \\
\hline & 0.89 & 1.20 & 9.4 & 28.5 & 780 & & 56.3 & & & 0.99 & & & 222.0 & 0.68 & $<0.05$ & 8.6 & 0.390 & 0.36 & 2.3 & & & 17.6 & & 68.4 & & \\
\hline 1010 & 0.80 & 1.29 & 12.5 & 27.1 & 820 & 10.6 & 54.7 & $<0.002$ & 0.02 & 0.92 & 1 & 1.70 & 240.0 & 0.90 & $<0.05$ & 10.6 & 0.497 & 0.34 & 2.5 & 119 & 1. & 20.1 & 77 & 89.7 & 25 & FA04 \\
\hline 1011 & 0.42 & 1.55 & 29.8 & 18.9 & 1930 & 22.8 & 79.1 & $<0.002$ & 0.02 & 1.47 & & 5.40 & 324.0 & 2.40 & 0.05 & 19.4 & 0.490 & 0.48 & 5.5 & 107 & & 24.3 & 70 & 95.7 & 3 & \\
\hline & 0.91 & 1.22 & 23.9 & 21.4 & & 23.3 & 83.4 & $<0.002$ & 0.04 & 1.72 & & & 294.0 & 1.60 & 0.05 & 21.4 & 0.436 & 0.51 & 6.2 & & & \begin{tabular}{|l|}
22.8 \\
\end{tabular} & 1 & 57.0 & & \\
\hline 101 & 0.69 & 1.61 & 38.4 & 19.6 & 3110 & 20.5 & 92.9 & \begin{tabular}{|l|}
$<0.002$ \\
\end{tabular} & 0.02 & 1.38 & 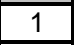 & 5.40 & 425.0 & 2.66 & 0.05 & 26.8 & 0.555 & 0.54 & 6.7 & 12 & 1 & 30.8 & 8 & 56.4 & 37 & 7731 \\
\hline & 0.70 & 1.65 & 49.5 & 15.5 & 2270 & 23.4 & 98.2 & & 0.02 & 2.00 & & 4.70 & 444.0 & 3.68 & $<0.05$ & 19.6 & 0.626 & 0.55 & 5.3 & & & 24.3 & 8 & 40.1 & 20 & \\
\hline 1016 & 0.65 & 1.44 & 39.9 & 20.0 & 2720 & 21.3 & 87.1 & \begin{tabular}{|c|}
$<0.002$ \\
\end{tabular} & 0.02 & 1.26 & 1 & 4.40 & 367.0 & 2.78 & $<0.05$ & 20.9 & 0.564 & 0.50 & 6.0 & $11 \mathrm{~s}$ & 1.6 & 28.4 & 80 & 63.0 & 22 & FA04030731 \\
\hline 1017 & 0.79 & 1.32 & 26.6 & 21.7 & 2200 & 23.8 & 86.3 & $<0.002$ & 0.03 & 1.30 & & 3.90 & 339.0 & 1.67 & $<0.05$ & 23.6 & 0.459 & 0.58 & 6.3 & 126 & 1.5 & 24.6 & 79 & 49.1 & 33 & FA04030731 \\
\hline
\end{tabular}


Table 1. Location and New Trace Element Geochemical Results for 902 Stream-Sediment Samples collected in the Livengood Area, Livengood Quadrangle, Alaska IS = Insufficient Sample for Analysis

\begin{tabular}{|c|c|c|c|c|c|c|c|c|c|c|c|c|c|c|c|c|c|c|c|c|c|c|c|c|c|c|c|}
\hline MPLE & TM E & TM N & $\begin{array}{c}\mathrm{Au} \\
\mathrm{ppm}\end{array}$ & \begin{tabular}{|c|}
$\mathrm{Ag}$ \\
$\mathrm{ppm}$
\end{tabular} & $\begin{array}{l}\mathrm{Al} \\
\% \\
\end{array}$ & $\begin{array}{c}\text { As } \\
\text { ppm }\end{array}$ & $\begin{array}{l}\mathrm{Ba}^{*} \\
\mathrm{ppm}\end{array}$ & \begin{tabular}{|c|}
$\mathrm{Be}$ \\
$\mathrm{ppm}$
\end{tabular} & $\begin{array}{c}\mathrm{Bi} \\
\mathrm{ppm}\end{array}$ & $\begin{array}{c}\mathrm{Ca} \\
\% \\
\end{array}$ & \begin{tabular}{|c|}
$\mathrm{Cd}$ \\
$\mathrm{ppm}$
\end{tabular} & $\begin{array}{l}\mathrm{Ce} \\
\mathrm{ppm}\end{array}$ & $\begin{array}{l}\text { Co } \\
\text { ppm }\end{array}$ & $\begin{array}{l}\mathrm{Cr}^{\star} \\
\mathrm{ppm}\end{array}$ & $\begin{array}{c}\text { Cs } \\
\text { ppm }\end{array}$ & $\begin{array}{c}\mathrm{Cu} \\
\mathrm{ppm}\end{array}$ & $\begin{array}{c}\mathrm{Fe} \\
\% \\
\end{array}$ & $\begin{array}{c}\mathrm{Ga} \\
\mathrm{ppm}\end{array}$ & $\begin{array}{c}\mathrm{Ge} \\
\mathrm{ppm}\end{array}$ & $\begin{array}{c}\mathrm{Hf} \\
\mathrm{ppm}\end{array}$ & $\begin{array}{l}\mathrm{Hg} \\
\mathrm{ppm}\end{array}$ & $\begin{array}{c}\text { In } \\
\text { ppm }\end{array}$ & $\begin{array}{l}\mathrm{K} \\
\% \\
\end{array}$ & $\begin{array}{c}\mathrm{La} \\
\mathrm{ppm}\end{array}$ & \begin{tabular}{|c|}
$\mathrm{Li}$ \\
$\mathrm{ppm}$
\end{tabular} & $\begin{array}{c}\mathrm{Mg} \\
\%\end{array}$ & $\begin{array}{l}\mathrm{Mn} \\
\mathrm{ppm}\end{array}$ \\
\hline 018 & 8748 & 0623 & $<0.005$ & 0.36 & & 17.0 & 660 & 2.19 & 0.42 & & \begin{tabular}{|l|}
0.18 \\
\end{tabular} & 147.0 & 14.1 & & 6.72 & 16.4 & 3.33 & 16.50 & 0.17 & \begin{tabular}{|l|}
2.1 \\
\end{tabular} & 0.10 & & 1.40 & 74.9 & 27.6 & & 829 \\
\hline 102 & & & & & 6.06 & 14.6 & $\overline{00}$ & $\overline{58}$ & $\overline{0.2}$ & 72 & \begin{tabular}{|l|}
0.22 \\
\end{tabular} & 122.0 & 15. & 91 & $\overline{4.4 !}$ & 5. & 3.34 & 14.70 & 0.15 & 2.6 & 0.22 & 0.050 & 1.30 & 64.6 & 26.2 & 0.98 & 63 \\
\hline & & & 116 & & & .2 & 30 & 55 & 0.24 & & 14 & 120.0 & 3.6 & & 4.1 & 5.4 & 3.28 & 14.60 & 0.17 & 2.7 & 0.13 & 0.055 & 1.32 & 5.2 & 4.9 & 1.00 & 89 \\
\hline & & & & & & & & & & & & & & & 32 & & 2.86 & 13.40 & & 20 & & & 131 & & & 095 & \\
\hline 1057 & & & .013 & 14 & 5.27 & 8.6 & 880 & .09 & .18 & .91 & \begin{tabular}{|l|l|}
0.34 \\
\end{tabular} & 54.4 & 8.1 & 7 & 2.67 & 20.6 & 2.31 & 13.80 & 0.13 & 1.9 & 0.10 & 0.039 & 1.23 & 27.7 & 3.7 & 0.67 & 81 \\
\hline & & & 005 & $\overline{12}$ & \begin{tabular}{|l|l|}
5.74 \\
\end{tabular} & 12.0 & 650 & .33 & 0.19 & 0.70 & \begin{tabular}{|l|l|}
0.18 \\
\end{tabular} & 68.0 & 11. & $\overline{82}$ & 3.60 & 19.8 & 2.93 & 16.30 & 0.16 & 2.0 & 0.15 & 0.044 & 1.53 & 32.1 & 27.2 & 0.67 & 295 \\
\hline & & & & $\overline{12}$ & \begin{tabular}{|l|l|}
5.47 \\
\end{tabular} & 6.3 & 570 & 0.98 & & 0.88 & \begin{tabular}{|l|}
0.16 \\
\end{tabular} & 56.6 & 7.7 & & 2.65 & $\overline{48}$ & 2.41 & 11.90 & $\overline{0.10}$ & 1.6 & 0.14 & 0.035 & 1.24 & 269 & 22.3 & 0.68 & 297 \\
\hline & & & 0.011 & 09 & 5.82 & 9.0 & 800 & 18 & 0.16 & 1.21 & \begin{tabular}{|l|}
0.29 \\
\end{tabular} & 53.2 & 15.5 & 7 & 2.51 & 5.8 & 2.84 & 14.05 & 0.14 & 2.0 & 0.08 & $\overline{041}$ & 1.30 & 27.0 & 24.4 & 0.82 & 346 \\
\hline & & & & & \begin{tabular}{|l|}
3.33 \\
\end{tabular} & & & & & 0.99 & & & & & & & & 8.08 &.$\angle 0$ & & 0.38 & & 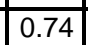 & & & \begin{tabular}{|l}
0.47 \\
\end{tabular} & \\
\hline & & & 035 & 67 & \begin{tabular}{|l|l}
7.80 \\
\end{tabular} & 3.5 & 30 & 36 & 23 & .06 & 32 & $\overline{51.1}$ & 20. & $\overline{05}$ & 10.75 & 12.2 & 4.60 & 18.10 & 0.17 & 1.7 & 0.15 & 0.062 & 1.38 & 26.5 & 40.2 & 1.29 & 120 \\
\hline 10 & & & 0.032 & 0.65 & \begin{tabular}{|l}
6.36 \\
\end{tabular} & 13.6 & 740 & 1.24 & 0.23 & 0.78 & \begin{tabular}{|l|}
0.27 \\
\end{tabular} & 50.0 & 17.4 & 106 & 9.60 & 41.2 & 3.77 & 17.95 & 0.18 & 1.8 & 0.21 & .057 & 1.25 & 24.8 & 39.2 & 1.02 & 726 \\
\hline & & & & & \begin{tabular}{|l|}
5.47 \\
\end{tabular} & & & & & & & & & & 3.01 & & & 14.30 & & 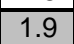 & & & \begin{tabular}{|l|}
1.27 \\
\end{tabular} & & & & \\
\hline & & & 0.010 & 16 & \begin{tabular}{|l|l|}
6.10 \\
\end{tabular} & 9.1 & $\overline{00}$ & 31 & 0.27 & 1.03 & & $\sqrt{93}$ & 14. & & 3.9 & 20.3 & 2.94 & 15.85 & 0.16 & 2.1 & 0.16 & 0.050 & 1.43 & 0. & 2.5 & 1100 & 96 \\
\hline & & & $\overline{0}$ & 0.16 & 5.68 & 12.8 & 700 & 1.26 & 0.51 & 1.24 & \begin{tabular}{|l|}
0.22 \\
\end{tabular} & 60.1 & 13. & 8 & 2.91 & 23.9 & 2.76 & 14.20 & 0.15 & 1.9 & 0.27 & .045 & 1.30 & 29.9 & 23.2 & 0.93 & 532 \\
\hline & & & & 20 & 5.66 & 6.8 & & & & 1.28 & & & & & & & & 14.40 & & 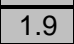 & 0.18 & & \begin{tabular}{|l|}
1.32 \\
\end{tabular} & & 22.8 & & 739 \\
\hline & & & 0.029 & 0.22 & 5.70 & 46 & 30 & 1.1 & $0.6 \mathrm{C}$ & 1.13 & 0.26 & $\overline{6}$ & 12. & & 3.47 & 24. & 2.69 & 14.35 & 0.1 & 1.9 & $<0.01$ & 054 & 1.26 & 32. & 22.2 & 0.85 & 599 \\
\hline & & & & & \begin{tabular}{|l|}
6.41 \\
\end{tabular} & & & & & 77 & 06 & & 22. & 102 & 11.55 & 1. & 3.65 & 16.15 & 0.17 & 1.7 & 0.65 & .061 & 1.25 & 30.7 & 45.6 & \begin{tabular}{|l|l}
0.97 \\
\end{tabular} & 315 \\
\hline & & & 0.010 & 52 & & & & $\overline{12}$ & & .05 & & & 16. & & 6.07 & & & 14.95 & 014 & $\sqrt{17}$ & & & & & & & \\
\hline & & & 0.012 & 0.94 & \begin{tabular}{|l|l|}
6.78 \\
\end{tabular} & 14.2 & 770 & 1.15 & 0.20 & 1.08 & \begin{tabular}{|l|l|}
1.44 \\
\end{tabular} & 57.5 & 22.6 & 106 & 7.29 & 44.9 & 3.78 & 15.85 & 0.16 & 1.9 & 0.40 & 0.058 & 1.32 & 29.8 & \begin{tabular}{|l|l|}
46.7 \\
\end{tabular} & 1.15 & 1380 \\
\hline & & & & 28 & .01 & 7.8 & 700 & & & 1.02 & \begin{tabular}{|l|l|}
0.33 \\
\end{tabular} & 5 & 14.9 & & 5.1 & 35. & 3.08 & 16.10 & 0.1 & 1. & 0.28 & .050 & 1.36 & 29.8 & 34.6 & 1.05 & 746 \\
\hline & & & & & 64 & 1 & 720 & & 0.17 & 94 & & & & & 5. & 33 & 3.8 & b. & & 1. & 0.5 & & 1. & & 43.2 & 1.2 & 445 \\
\hline & & & & .44 & 5.93 & 12.4 & 600 & 17 & $\overline{0.1}$ & 95 & 88 & & 17. & 1 & 5.08 & 36 & 3.55 & 15.95 & 0.1 & 2. & 0.77 & .060 & \begin{tabular}{|l|} 
\\
\end{tabular} & 30.4 & 40.6 & 1.14 & 903 \\
\hline & & & & & & 9.9 & 6 & & & $\overline{04}$ & & & & & & 9 & 3.39 & 5.25 & 0. & & .58 & .051 & 1.28 & 31.3 & 37.4 & 1.1 & 826 \\
\hline & & & & 20 & 5.78 & 11.5 & 830 & & 0. & 39 & 30 & 58.3 & 14. & 85 & 3.6 & 21. & 3.01 & 5.10 & $\overline{0.1}$ & 1. & 51 & & \begin{tabular}{|l|}
1.32 \\
\end{tabular} & 30.1 & 27.1 & \begin{tabular}{|l|}
0.91 \\
\end{tabular} & 3570 \\
\hline & & & & & \begin{tabular}{|l|l|}
6.17 \\
\end{tabular} & 8. & 700 & & & 1.24 & & & & & & 29. & 3.08 & 4.65 & 0.1 & 1. & 0.50 & .044 & 1.32 & 30.6 & 31.4 & \begin{tabular}{|l|l|}
1.05 \\
\end{tabular} & 844 \\
\hline & & & 0.152 & 0.33 & 6.31 & 8.5 & 750 & & & 1.28 & 69 & & 14. & & & 27. & 3.20 & 4.40 & 0.15 & & 0.48 & .052 & $\begin{array}{l}1.29 \\
\end{array}$ & 30.9 & 32.9 & 1.08 & 1480 \\
\hline & & & & 0.38 & \begin{tabular}{|l|}
6.49 \\
\end{tabular} & 10.8 & 710 & 1.36 & & 1.22 & \begin{tabular}{|l|}
0.69 \\
\end{tabular} & & & & & 618 & 3.72 & 6.50 & 017 & 2. & 0.47 & & 1.49 & 34.7 & 2.1 & 1.18 & 895 \\
\hline & & & 0 & 29 & .71 & 7. & 660 & & & .23 & & 9. & 13. & & 3.4 & 22. & 2.82 & 4.15 & & 1. & 0.41 & .042 & \begin{tabular}{|l|} 
\\
\end{tabular} & 32.8 & 30.4 & 1.00 & 821 \\
\hline & & & & & 6.23 & 8 & & & & 1.30 & & & & & & & & & & & & & 1.33 & 34 & 33.1 & 1.10 & 035 \\
\hline & & & 0.039 & 23 & 5.99 & & 670 & & & .41 & $\mid 032$ & & & & 3. & 19 & 3.1 & 44.45 & & 2. & $\overline{0.2}$ & .04 & 1.26 & 42 & 29 . & 1.07 & 792 \\
\hline & & & 0.023 & 2.63 & 5.34 & 8.4 & 560 & & $\overline{0.2}$ & 1.32 & \begin{tabular}{|l|}
3.83 \\
\end{tabular} & & 14. & & 8.4 & 2. & 2.80 & 13.95 & 0.13 & 1. & .90 & 136 & \begin{tabular}{|l|}
1.13 \\
\end{tabular} & 26.7 & 17.9 & 0.91 & 330 \\
\hline & & & & 1.46 & 5.49 & 39.0 & 660 & & & & & & & & & & & & & 1. & & & 1.10 & 7.4 & 9.4 & 0.79 & 831 \\
\hline & & & & & 05 & & 890 & & & 13 & & & & & 5.3 & 9. & 4.05 & 6.10 & & 2. & 0.92 & 102 & 1.32 & 32.5 & 34.6 & 0.95 & 7160 \\
\hline & & & & 1.07 & 5.35 & & 63 & & 0. & 1.38 & 2.01 & & & & 4.9 & & 2.5 & 13.50 & & 1. & & 1069 & 1.17 & 28.0 & 32.1 & 0.86 & 964 \\
\hline & & & & & & & & & & & & & & & & & & & & & & & & & 07 & 10 & \\
\hline & & & & & 5. & 29.7 & 740 & & & 1.20 & & & & & & & 2.97 & 14.50 & & 1. & .50 & .065 & 1.30 & 31.4 & 31.7 & 0.87 & 2300 \\
\hline & & & 006 & 15 & 5.21 & 8.2 & 740 & & 0. & 1.36 & \begin{tabular}{|l|}
0.31 \\
\end{tabular} & & 10 & & 2.3 & & 2.54 & 12.90 & 0.15 & 2.2 & .11 & .037 & \begin{tabular}{|l|}
1.17 \\
\end{tabular} & 31.4 & 7.6 & \begin{tabular}{|l|}
0.77 \\
\end{tabular} & 522 \\
\hline & & & & & & & & & & & & & & & & & & & & & & & & & & 7 70 & 88 \\
\hline 11 & 5565 & 32 & .005 & 16 & 5.38 & 8.8 & 780 & & 0. & 1.39 & 34 & & 12 & & 2.5 & 20.9 & 2.72 & 13.10 & & 2.2 & 0.1 & 0.040 & 1.19 & 33.5 & 9.3 & 0.79 & 882 \\
\hline & & & & 0.12 & 5.24 & 7.4 & 750 & & & 1.58 & & & 11 & & 2. & & 2.65 & & & 2.3 & .07 & .040 & \begin{tabular}{|l|}
1.17 \\
\end{tabular} & 36.7 & $8.7 \mid$ & 0.83 & 539 \\
\hline & & & & & & & 660 & & & & & & & & & & & & & & & & & 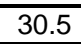 & & 0.66 & 36 \\
\hline 112 & & 7266923 & 0.005 & 0.14 & 5.25 & 8.5 & 750 & 1.1 & 0.14 & $\overline{1.3 .}$ & 0.34 & 58 & 12. & 75 & 2.56 & 24. & 2.64 & 13.00 & & 2.0 & 0.09 & & 1.17 & 30.3 & 8.2 & 0.76 & 611 \\
\hline & & & & & & 11.1 & 780 & & & & & & 14.1 & & & 28.4 & 3.03 & & & & 0.13 & 0.043 & 1.29 & 36.1 & 22.0 & 0.79 & 330 \\
\hline
\end{tabular}


Table 1. Location and New Trace Element Geochemical Results for 902 Stream-Sediment Samples collected in the Livengood Area, Livengood Quadrangle, Alaska IS = Insufficient Sample for Analysis

\begin{tabular}{|c|c|c|c|c|c|c|c|c|c|c|c|c|c|c|c|c|c|c|c|c|c|c|c|c|c|c|}
\hline SAMPLE & $\begin{array}{l}\text { Mo } \\
\text { ppm }\end{array}$ & $\begin{array}{c}\mathrm{Na} \\
\%\end{array}$ & \begin{tabular}{|l|}
$\mathrm{Nb}$ \\
$\mathrm{ppm}$
\end{tabular} & $\begin{array}{c}\mathrm{Ni} \\
\mathrm{ppm}\end{array}$ & $\begin{array}{c}\mathrm{P} \\
\mathrm{ppm}\end{array}$ & $\begin{array}{l}\mathrm{Pb} \\
\mathrm{ppm}\end{array}$ & $\begin{array}{l}\mathrm{Rb} \\
\mathrm{ppm}\end{array}$ & $\begin{array}{l}\mathrm{Re} \\
\mathrm{ppm}\end{array}$ & $\begin{array}{l}\mathrm{S} \\
\%\end{array}$ & $\begin{array}{c}\mathrm{Sb} \\
\mathrm{ppm}\end{array}$ & \begin{tabular}{|c|} 
Se \\
ppm
\end{tabular} & $\begin{array}{l}\text { Sn* } \\
\text { ppm }\end{array}$ & $\begin{array}{c}\mathrm{Sr} \\
\mathrm{ppm}\end{array}$ & $\begin{array}{l}\mathrm{Ta}^{\star} \\
\mathrm{ppm}\end{array}$ & $\begin{array}{c}\mathrm{Te} \\
\mathrm{ppm}\end{array}$ & \begin{tabular}{|c|} 
Th \\
ppm
\end{tabular} & $\begin{array}{l}\mathrm{Ti}^{\star} \\
\%\end{array}$ & $\begin{array}{c}\mathrm{TI} \\
\mathrm{ppm}\end{array}$ & $\begin{array}{c}\mathrm{U} \\
\mathrm{ppm}\end{array}$ & $\begin{array}{c}\mathrm{V} \\
\mathrm{ppm}\end{array}$ & $\begin{array}{l}W^{*} \\
\text { ppm }\end{array}$ & $\begin{array}{c}\mathrm{Y} \\
\mathrm{ppm}\end{array}$ & $\begin{array}{c}\mathrm{Zn} \\
\mathrm{ppm}\end{array}$ & $\begin{array}{l}\mathrm{Zr}^{*} \\
\mathrm{ppm}\end{array}$ & $\begin{array}{c}\text { Pulp Wt } \\
\text { grams }\end{array}$ & $\begin{array}{c}\text { Lab } \\
\text { Report }\end{array}$ \\
\hline 1018 & 0.72 & \begin{tabular}{|l|l|}
1.44 \\
\end{tabular} & 29.6 & 21.6 & 2260 & 19.6 & 90.3 & $<0.002$ & 0.02 & 1.24 & 1 & 4.00 & 354.0 & 2.14 & $<0.05$ & 22.7 & 0.528 & 0.48 & 5.3 & 117 & 1.7 & 24.6 & 77 & 58.5 & 20 & FA04030731 \\
\hline 020 & 0.68 & 28 & 24.1 & 26.4 & $\overline{460}$ & 15.5 & $\overline{2.3}$ & 0.002 & 0.02 & 1.22 & 1 & 2.60 & 67.0 & 1.81 & 0.05 & 18.9 & 0.567 & 0.43 & 4.8 & 123 & 2.0 & 21.4 & 80 & 75.4 & 23 & FA04030731 \\
\hline 1021 & 0.67 & 1.32 & 23.2 & 26.1 & 1360 & 14.6 & 67.4 & $<0.002$ & 0.02 & 1.59 & 1 & 2.60 & 266.0 & 1.69 & $<0.05$ & \begin{tabular}{|l|}
19.4 \\
\end{tabular} & 0.588 & 0.42 & 4.5 & 125 & 1.5 & 21.6 & 78 & 77.5 & 2 & FA04030731 \\
\hline 022 & 0.62 & .30 & \begin{tabular}{|l|}
13.7 \\
\end{tabular} & 27.4 & 1060 & 12.0 & 59.1 & $<0.002$ & 0.03 & 1.04 & & 2.00 & 257.0 & \begin{tabular}{|l|}
0.93 \\
\end{tabular} & $<0.05$ & \begin{tabular}{|l|}
14.3 \\
\end{tabular} & 0.459 & 0.37 & 3.2 & 111 & 1.1 & 17.9 & 77 & 63.6 & 8 & FA04030731 \\
\hline 1057 & 1.04 & .97 & 9.7 & 21.9 & 670 & 12.5 & 60.2 & $<0.002$ & 0.03 & 1.02 & 1 & 1.90 & 160.5 & \begin{tabular}{|l|}
0.67 \\
\end{tabular} & 0.05 & \begin{tabular}{|l|}
8.0 \\
\end{tabular} & 0.376 & 0.43 & 2.5 & 107 & 1.2 & 13.0 & 80 & 62.5 & 18 & FA04030731 \\
\hline 60 & 2.27 & 0.70 & 9.7 & 30.3 & 650 & 14.5 & 71.9 & $<0.002$ & 0.03 & 1.34 & & 4.40 & 131.5 & 0.72 & 0.06 & 8.7 & 0.377 & 0.45 & 2.3 & 122 & 1.4 & 12.9 & 84 & 61.8 & 34 & FA04030731 \\
\hline & $\overline{0.95}$ & 84 & 7.7 & 19.4 & 610 & & 52.3 & $<0002$ & 0.03 & 0.90 & & 1.60 & 143.5 & 0.54 & $<0.05$ & 7.0 & 0.342 & 0.36 & 1.9 & 104 & & & & & & \\
\hline 1064 & 0.97 & 1.16 & 9.8 & 25.8 & 770 & 12.0 & 61.1 & $<0.002$ & 0.03 & 1.15 & 1 & 1.70 & 196.0 & 0.69 & $<0.05$ & 7.9 & 0.374 & 0.40 & 2.1 & 112 & 1.0 & 14.2 & 91 & 60.1 & 22 & FA04030731 \\
\hline 1065 & 4.99 & \begin{tabular}{|l|}
0.58 \\
\end{tabular} & 5.1 & 24.7 & 1510 & 10.3 & 37.6 & $<0.002$ & 0.12 & 1.29 & 2 & 1.20 & 123.5 & 0.33 & 0.05 & 5.7 & 0.207 & 0.24 & 1.6 & 106 & 0.6 & 15.9 & 305 & 35.3 & 1 & FA04030731 \\
\hline 1073 & 1.27 & \begin{tabular}{|l|}
1.06 \\
\end{tabular} & 9.7 & 42.7 & 1170 & 17.4 & 78.8 & $<0.002$ & 0.06 & 1.12 & 1 & 2.30 & 166.0 & \begin{tabular}{|l|}
0.60 \\
\end{tabular} & 0.07 & 7.2 & 0.419 & 0.54 & 1.9 & 164 & 1.6 & 17.6 & 161 & 57.5 & & \\
\hline 75 & 1.20 & \begin{tabular}{|l|}
0.83 \\
\end{tabular} & 8.8 & 42.5 & 940 & 17.5 & 75.2 & $<0.002$ & 0.05 & 1.07 & 1 & 2.10 & 128.0 & \begin{tabular}{|l|}
0.61 \\
\end{tabular} & 0.08 & 7.3 & 0.400 & 0.56 & 2.0 & 159 & 1.8 & \begin{tabular}{|l|}
16.1 \\
\end{tabular} & 141 & 57.1 & 2 & FA04030731 \\
\hline 1077 & 0.62 & 1.11 & 9.8 & 29.1 & 620 & 11.2 & 62.7 & $<0.002$ & 0.03 & 0.97 & 1 & 2.10 & 183.0 & 0.65 & $<0.05$ & 7.7 & 0.396 & 0.39 & 1.8 & 111 & 1.3 & 13.8 & 92 & 59.4 & 2 & FA04030731 \\
\hline 78 & 0.66 & 1.14 & 11.2 & 34.4 & 620 & 12.0 & 67.8 & $<0.002$ & 0.03 & 0.98 & & 2.10 & 169.5 & \begin{tabular}{|l|}
0.75 \\
\end{tabular} & 0.05 & 8.4 & 0.464 & 0.44 & 2.0 & 133 & & 14.9 & 92 & 67.0 & & \\
\hline 79 & 0.66 & 1.13 & 10.0 & 30.3 & 630 & 11.5 & 61.4 & $<0.002$ & 0.03 & 1.08 & & 2.30 & 186.5 & 0.70 & $<0.05$ & 8.2 & 0.415 & 0.42 & 2.0 & 116 & 1.7 & 14.8 & 94 & 59.4 & 2 & FA04030731 \\
\hline 8. & 0.59 & 1.06 & 9.8 & 31.3 & 580 & 12.5 & 65.6 & $<0.002$ & 0.05 & 1.07 & & 2.30 & 184.0 & \begin{tabular}{|l|}
0.65 \\
\end{tabular} & 0.05 & 7.5 & 0.402 & 0.43 & 2.0 & 111 & 1.3 & 14.6 & 100 & 60.6 & & FA04030731 \\
\hline & 0.79 & 1.01 & 9.9 & 30.5 & 590 & 11.3 & 61.8 & $<0.002$ & 0.02 & 1.02 & & 2.80 & 167.0 & \begin{tabular}{|l|l|}
0.67 \\
\end{tabular} & 0.06 & 7.8 & 0.424 & 0.45 & 2.1 & 118 & & 14.8 & 69 & 60. & & 0731 \\
\hline 1 & 1.28 & \begin{tabular}{|l|}
0.71 \\
\end{tabular} & 7.7 & 47.8 & 970 & 27.6 & 85.0 & $<0.002$ & 0.07 & 2.03 & & 2.20 & 125.0 & 0.54 & 0.08 & 6.9 & 0.332 & 0.62 & 2.0 & 148 & 1.1 & 23.6 & 258 & 57.5 & 1 & FA04030731 \\
\hline & 1.08 & \begin{tabular}{|l|}
1.02 \\
\end{tabular} & 8.7 & 44.3 & 900 & 18.4 & 66.0 & $<0.002$ & 0.06 & 1.47 & & 1.80 & 154.0 & 0.56 & 0.06 & 6.5 & 0.415 & 0.43 & 1.8 & 142 & & 21.6 & 244 & 56.7 & & FA04030731 \\
\hline & 1.22 & \begin{tabular}{|l|}
0.98 \\
\end{tabular} & 9.2 & 46.1 & 940 & 39.3 & 74.5 & $<0.002$ & 0.05 & 2.25 & & 3.20 & 158.0 & \begin{tabular}{|l|}
0.57 \\
\end{tabular} & 0.08 & 6.9 & 0.419 & 0.52 & 2.0 & 151 & 1. & 21.9 & 192 & 65. & 2 & FA04030731 \\
\hline$\overline{888}$ & 0.88 & 1.06 & 10.1 & 41.7 & 630 & 13.7 & 72.1 & $<0.002$ & 0.03 & 0.90 & & 2.60 & 165.5 & 0.70 & 0.05 & 7.2 & 0.442 & 0.41 & 2.0 & 135 & 13 & 17.0 & 99 & 63.4 & & FA04030731 \\
\hline 89 & 95 & 1.11 & 10.0 & 4 & 750 & 20.1 & 63.5 & $<0.002$ & 0.03 & 1.43 & & 2.10 & 147.0 & 0.70 & 0.07 & 6.5 & 0.497 & 0.41 & 1.8 & 156 & & 18.5 & 126 & 59. & & 0731 \\
\hline 90 & 1.42 & \begin{tabular}{|l|}
1.08 \\
\end{tabular} & 10.2 & 49.6 & 730 & 27.6 & 62.0 & $<0.002$ & 0.03 & 2.03 & 1 & 6.90 & 166.5 & \begin{tabular}{|l|}
0.66 \\
\end{tabular} & 0.07 & 6.9 & 0.500 & 0.40 & 2.0 & 153 & 1.5 & 19.4 & 139 & 63.6 & 3 & FA04030731 \\
\hline 91 & 0.86 & \begin{tabular}{|l|l|}
1.14 & \\
\end{tabular} & 10.2 & 42.7 & 710 & 25.2 & 61.0 & $<0.002$ & 0.02 & 1.80 & & 4.70 & 166.5 & \begin{tabular}{|l|}
0.67 \\
\end{tabular} & 0.06 & 7.3 & 0.486 & 0.39 & 2.0 & 140 & 1.5 & 19.5 & 116 & 65.0 & & FA04030731 \\
\hline & 0.64 & \begin{tabular}{|l|}
1.09 \\
\end{tabular} & 9.5 & 37.4 & 780 & 11 & 60.6 & $<0.002$ & 0.05 & 3.09 & & 1.90 & 226.0 & 0.62 & 0.06 & 7.2 & 0.368 & 0.40 & 2.0 & & & 16.4 & 76 & 60.9 & & 0731 \\
\hline 1093 & 0.69 & 1.12 & 9.4 & 38.6 & 740 & 22.0 & 60.9 & $<0.002$ & 0.04 & 1.49 & & 1.80 & 196.5 & 0.65 & $<0.05$ & 7.1 & 0.423 & 0.38 & 1.9 & 125 & 1.1 & 18.2 & 104 & 57.4 & $?$ & FA04030731 \\
\hline 1094 & 0.72 & 1.12 & 9.5 & 39.1 & 800 & 20.4 & 62.0 & $<0.002$ & 0.05 & 1.43 & & 1.80 & 207.0 & \begin{tabular}{|l|l|}
0.67 \\
\end{tabular} & 0.06 & 7.3 & 0.407 & 0.37 & 1.9 & 120 & 1. & 17.6 & 104 & 58.3 & & FA04030731 \\
\hline & & 0.99 & 10.0 & 50.0 & 910 & 16.8 & 67.4 & $<0.002$ & 0.04 & 1.67 & & 2.20 & 184.5 & 0.66 & 0.07 & 6.9 & 0.468 & 0.48 & 1.8 & & & \begin{tabular}{|l|l}
25.7 \\
\end{tabular} & 119 & 63 & & \\
\hline & 0.74 & \begin{tabular}{|l|l|}
1.14 \\
\end{tabular} & 10.0 & 37.1 & 660 & 24.2 & 60.2 & $<0.002$ & 0.03 & 1.61 & & 1.70 & 195.0 & 0.66 & 0.05 & 8.2 & 0.442 & 0.38 & 1.9 & 117 & & 17.1 & 96 & 62. & & FA04030731 \\
\hline & 0.76 & 1.20 & 10.6 & 40.4 & 750 & 27.3 & 61.7 & $<0.002$ & 0.03 & 1.85 & & 2.70 & 201.0 & 0.72 & 0.05 & 8.1 & 0.473 & 0.41 & 2.1 & 1 & & 17.6 & 107 & 64. & & FA04030731 \\
\hline & 0.65 & \begin{tabular}{|l|}
1.24 \\
\end{tabular} & 12.0 & 36.4 & 740 & 22.1 & 59.1 & $<0.002$ & 0.03 & 1.57 & & 1.80 & 204.0 & \begin{tabular}{|l|}
0.88 \\
\end{tabular} & $<0.05$ & 9.6 & 0.540 & 0.35 & 2.4 & 28 & & 19.8 & 92 & 73. & & FA04030731 \\
\hline 1102 & 0.76 & 0.80 & \begin{tabular}{|l|}
8.2 \\
\end{tabular} & 40.8 & 680 & 69.1 & 62.2 & $<0.002$ & 0.07 & 9.24 & & 2.30 & 208.0 & 0.52 & 0.06 & 6.1 & 0.374 & 0.45 & 1.9 & 123 & 1.0 & 18.0 & 273 & 51.8 & 1 & FA04030731 \\
\hline & 0.64 & \begin{tabular}{|l|}
0.85 \\
\end{tabular} & 8.7 & 35.4 & 740 & 38.5 & 62.1 & $<0.002$ & 0.06 & 2.18 & & 1.90 & 171.5 & \begin{tabular}{|l|}
0.58 \\
\end{tabular} & 0.06 & 6.8 & 0.371 & 0.46 & 1.9 & 110 & 1 & 16.2 & 142 & 57.3 & & FA04030731 \\
\hline & & \begin{tabular}{|l|}
1.02 \\
\end{tabular} & 9.3 & & 820 & 53.7 & 65.2 & $<0.002$ & 0.03 & 6.17 & & 4.80 & 172.5 & 0.63 & 0.07 & 7.7 & 0.412 & 0.43 & 2.0 & 130 & & 19.3 & 192 & 74.0 & & FA04030731 \\
\hline 06 & 0.43 & \begin{tabular}{|l|l|}
0.97 \\
\end{tabular} & 7.9 & 34.9 & 660 & 30.9 & 60.4 & $<0.002$ & 0.10 & 2.83 & 1 & 1.90 & 199.0 & 0.54 & $<0.05$ & 7.0 & 0.349 & 0.40 & 1.6 & 104 & .8 & 16.2 & 152 & 51.4 & & FA04030731 \\
\hline 07 & 0.63 & \begin{tabular}{|l|}
1.02 \\
\end{tabular} & 9.3 & 34.5 & 710 & 28.9 & 63.1 & $<0.002$ & 0.07 & 2.20 & & 1. & 214.0 & 0.62 & $<0.05$ & 7.3 & 0.394 & 0.41 & 1.9 & 109 & 1.1 & \begin{tabular}{|l|}
15.3 \\
\end{tabular} & 131 & 57.4 & & FA04030731 \\
\hline & 0.63 & \begin{tabular}{|l|}
0.95 \\
\end{tabular} & 9.7 & 38.0 & 690 & & 67.1 & $<0.002$ & 0.06 & 2.16 & & 2. & 182.0 & \begin{tabular}{|l|}
0.68 \\
\end{tabular} & \begin{tabular}{|l|}
0.05 \\
\end{tabular} & 7.5 & 0.413 & 0.42 & 2.0 & 116 & & \begin{tabular}{|l|}
15.6 \\
\end{tabular} & 144 & 60.7 & & FA04030731 \\
\hline 11 & 0.92 & \begin{tabular}{|l|}
1.07 \\
\end{tabular} & 10.2 & 25.4 & 650 & 11.0 & 55.3 & $<0.002$ & 0.03 & 1.03 & 1 & 2.60 & 192.5 & 0.75 & 0.05 & 7.9 & 0.409 & 0.33 & 2.3 & 112 & 0 & 16.0 & 68 & 71.9 & 1 & FA04030731 \\
\hline & 1.36 & \begin{tabular}{|l|}
1.08 \\
\end{tabular} & 10.0 & 26.2 & 670 & 12 & 56.4 & $<0.002$ & 0.03 & 1.13 & & 1. & 185.0 & 0.70 & 0.05 & 7.9 & 0.401 & 0.39 & 2.3 & 119 & & 15.0 & 69 & 68.1 & & \\
\hline & 1.13 & 1.08 & \begin{tabular}{|l|}
10.2 \\
\end{tabular} & 26.7 & 690 & 12.0 & 56.8 & $<0.002$ & 0.03 & 1.12 & & 1. & 196.0 & \begin{tabular}{|l|}
0.71 \\
\end{tabular} & 0.05 & 8.6 & 0.402 & 0.36 & 2.7 & 118 & & 18.4 & 7. & 70.3 & 2 & FA04030731 \\
\hline 1122 & 0.92 & 1.20 & \begin{tabular}{|l|}
10.8 \\
\end{tabular} & 25.7 & 720 & 11.0 & 55.5 & $<0.002$ & 0.03 & 1.00 & -1 & 1.60 & 215.0 & \begin{tabular}{|l|}
0.71 \\
\end{tabular} & $<0.05$ & 9.6 & 0.442 & 0.38 & 2.4 & 116 & 1.2 & \begin{tabular}{|l|}
18.0 \\
\end{tabular} & 72 & 73.2 & 19 & FA04030731 \\
\hline & 1.95 & \begin{tabular}{|l|}
0.81 \\
\end{tabular} & \begin{tabular}{|l|}
9.7 \\
\end{tabular} & 30.3 & 730 & 14.9 & 58.1 & $<0.002$ & 0.02 & 1.34 & & 1.60 & 152.0 & 0.70 & \begin{tabular}{|l|}
0.09 \\
\end{tabular} & 8.5 & 0.378 & 0.48 & 3.4 & 118 & & \begin{tabular}{|l|}
16.2 \\
\end{tabular} & . & 67.0 & 3 & FA04030731 \\
\hline 1124 & 1.17 & 1.13 & 9.9 & 26.5 & 640 & 11.8 & 55.4 & $<0.002$ & 0.03 & 1.13 & 1 & 1.50 & 195.0 & 0.70 & 0.05 & 7.8 & 0.382 & 0.39 & 2.5 & 111 & 1.4 & 16.0 & 69 & 62.4 & 22 & FA0403 \\
\hline 1125 & 1.30 & 0.93 & 10.2 & 30.8 & 670 & 16.1 & 65.0 & $<0.002$ & \begin{tabular}{|l|}
0.02 \\
\end{tabular} & 1.14 & & 1.90 & 176.5 & 0.66 & 0.08 & $\begin{array}{ll}9.9 \\
\end{array}$ & \begin{tabular}{|l|}
0.384 \\
\end{tabular} & \begin{tabular}{|l|}
0.44 \\
\end{tabular} & 2.4 & 115 & 1.4 & 18.4 & 83 & 69.5 & 20 & FA04030731 \\
\hline
\end{tabular}


Table 1. Location and New Trace Element Geochemical Results for 902 Stream-Sediment Samples collected in the Livengood Area, Livengood Quadrangle, Alaska IS = Insufficient Sample for Analysis

\begin{tabular}{|c|c|c|c|c|c|c|c|c|c|c|c|c|c|c|c|c|c|c|c|c|c|c|c|c|c|c|c|}
\hline MPLE & TME & $\mathrm{MN}$ & $\begin{array}{c}\mathrm{Au} \\
\mathrm{ppm}\end{array}$ & \begin{tabular}{|l|}
$\mathrm{Ag}$ \\
$\mathrm{ppm}$
\end{tabular} & $\begin{array}{l}\text { Al } \\
\%\end{array}$ & $\begin{array}{l}\text { As } \\
\text { ppm }\end{array}$ & $\begin{array}{l}\mathrm{Ba}^{*} \\
\mathrm{ppm}\end{array}$ & \begin{tabular}{|c|}
$\mathrm{Be}$ \\
$\mathrm{ppm}$
\end{tabular} & $\begin{array}{c}\mathrm{Bi} \\
\mathrm{ppm}\end{array}$ & $\begin{array}{c}\mathrm{Ca} \\
\%\end{array}$ & \begin{tabular}{|l|}
$\mathrm{Cd}$ \\
$\mathrm{ppm}$
\end{tabular} & $\begin{array}{l}\mathrm{Ce} \\
\mathrm{ppm}\end{array}$ & $\begin{array}{l}\text { Co } \\
\text { ppm }\end{array}$ & $\begin{array}{l}\mathrm{Cr}^{\star} \\
\mathrm{ppm}\end{array}$ & $\begin{array}{c}\text { Cs } \\
\mathrm{ppm}\end{array}$ & $\begin{array}{c}\mathrm{Cu} \\
\mathrm{ppm}\end{array}$ & $\begin{array}{c}\mathrm{Fe} \\
\%\end{array}$ & $\begin{array}{c}\mathrm{Ga} \\
\mathrm{ppm}\end{array}$ & $\begin{array}{c}\text { Ge } \\
\mathrm{ppm}\end{array}$ & $\begin{array}{c}\mathrm{Hf} \\
\mathrm{ppm}\end{array}$ & $\begin{array}{l}\mathrm{Hg} \\
\mathrm{ppm}\end{array}$ & $\begin{array}{c}\text { In } \\
\text { ppm }\end{array}$ & $\begin{array}{l}\mathrm{K} \\
\%\end{array}$ & $\begin{array}{c}\mathrm{La} \\
\mathrm{ppm}\end{array}$ & \begin{tabular}{|c|}
$\mathrm{Li}$ \\
$\mathrm{ppm}$
\end{tabular} & $\begin{array}{c}\mathrm{Mg} \\
\%\end{array}$ & $\begin{array}{l}\mathrm{Mn} \\
\mathrm{ppm}\end{array}$ \\
\hline 128 & 7336 & & $<0.005$ & & & 9.1 & 820 & & & & & & & 76 & & 166 & 2.86 & & 0.15 & 21 & 0.10 & & 1.19 & 32.5 & \begin{tabular}{|l|}
20.0 \\
\end{tabular} & 0.82 & 585 \\
\hline & & & & & & 24 & & & & & & & & & & & & & & & & & 1.24 & 33.1 & \begin{tabular}{|l|}
20.2 \\
\end{tabular} & \begin{tabular}{|l|}
0.74 \\
\end{tabular} & 98 \\
\hline & & & 008 & 0.17 & & & 90 & & & & & & & 82 & & 28.9 & & & 0.15 & & & 037 & 0.83 & 26.7 & \begin{tabular}{|l|}
18.8 \\
\end{tabular} & 0.68 & 385 \\
\hline & & & & & & & & & & & & & & & & & & & 0.14 & & & & & 30.0 & 21.2 & & 859 \\
\hline & & & 010 & 0.26 & 81 & 1.5 & 90 & .27 & 21 & 1.22 & \begin{tabular}{|l|l|}
0.54 \\
\end{tabular} & 3.8 & $\overline{5.4}$ & 103 & 3.0 & 2.2 & 3.76 & 5.90 & 0.18 & 3.5 & 20 & .059 & 1.23 & 34.3 & 26.4 & 0.76 & $\overline{700}$ \\
\hline & & & .005 & 0.34 & .72 & $\overline{8.8}$ & 50 & 43 & 20 & 1.08 & 0.63 & & & 95 & $\overline{3.3}$ & 51.8 & .69 & 6.35 & 0.19 & 3.2 & & \begin{tabular}{|l|}
0.061 \\
\end{tabular} & 1.26 & 33.2 & \begin{tabular}{|l|}
27.2 \\
\end{tabular} & 0.71 & 882 \\
\hline & & & 019 & 0.26 & \begin{tabular}{|l|l|}
6.17 \\
\end{tabular} & & 90 & 51 & & & \begin{tabular}{|l|l|}
0.54 \\
\end{tabular} & & & & & & & & & & & & & & \begin{tabular}{|l|}
33.1 \\
\end{tabular} & & \\
\hline & & & 0.012 & 0.33 & \begin{tabular}{|l|}
6.30 \\
\end{tabular} & & 750 & .60 & & 1.12 & 0.73 & 8.7 & & 97 & 3.52 & 54.4 & 4.13 & 16.65 & 0.19 & 2.7 & & 0.066 & 1.18 & 34.3 & 30.0 & & 1045 \\
\hline & & & .005 & 0.19 & 21 & 0.6 & 920 & 63 & 17 & 1.69 & 0.41 & 4.1 & 3.4 & 86 & 2.85 & 31.3 & 3.46 & 15.00 & 0.18 & 2.0 & 0.14 & 0.050 & 1.29 & 37.4 & \begin{tabular}{|l|}
25.4 \\
\end{tabular} & 0.96 & 711 \\
\hline & & & .005 & \begin{tabular}{|l|l|}
0.09 \\
\end{tabular} & & & 370 & 68 & $\overline{09}$ & & \begin{tabular}{|l|}
0.33 \\
\end{tabular} & $\overline{96}$ & & & & & 161 & \begin{tabular}{|l|}
9.06 \\
\end{tabular} & & & & & 0.50 & 14.2 & \begin{tabular}{|l|}
13.4 \\
\end{tabular} & & 390 \\
\hline & & & 011 & 0.10 & \begin{tabular}{|l}
5.16 \\
\end{tabular} & 4 & 30 & 79 & 10 & 1.80 & 0.19 & 55.1 & 25.9 & & 1.79 & 20. & 3.50 & 11.15 & 0.14 & 1. & & \begin{tabular}{|l|l}
0.035 \\
\end{tabular} & 0.78 & 28.8 & \begin{tabular}{|l|l|}
15.7 \\
\end{tabular} & \begin{tabular}{|l}
3.17 \\
\end{tabular} & 635 \\
\hline & & & .005 & \begin{tabular}{|l|l|}
0.07 \\
\end{tabular} & 75 & & & & & 14 & 26 & 0.9 & 4.7 & & .28 & 23. & .53 & 11.10 & 0.12 & 1. & & \begin{tabular}{|l|l|}
0.036 \\
\end{tabular} & 0.58 & 17.3 & \begin{tabular}{|l|}
11.8 \\
\end{tabular} & 3.89 & 035 \\
\hline & & & .005 & .09 & 04 & & & 80 & & 90 & 22 & 7.8 & $\sqrt{33}$ & & 5 & & 92 & 0.90 & 0.14 & & & .039 & 0.73 & 23.8 & $\mid 16.2$ & & 688 \\
\hline & & & .005 & \begin{tabular}{|l|l|}
0.08 \\
\end{tabular} & 5.00 & 6.1 & 530 & .84 & .08 & 1.69 & 0.35 & 49.9 & 35.3 & 840 & 1.61 & 20.5 & 3.99 & 10.70 & 0.13 & 1.2 & .23 & 0.035 & \begin{tabular}{|l|l|}
0.74 \\
\end{tabular} & 24.1 & \begin{tabular}{|l|l|}
18.0 \\
\end{tabular} & \begin{tabular}{|l|}
3.94 \\
\end{tabular} & 1170 \\
\hline & & & 006 & \begin{tabular}{|l|l|}
0.11 \\
\end{tabular} & & & & & & & & & & & & 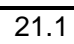 & & 12.20 & 0.14 & & & 0.039 & & 28.4 & \begin{tabular}{|l|l|}
18.2 \\
\end{tabular} & & 713 \\
\hline & & & 0.005 & 0.10 & & & 50 & .07 & & 65 & $\overline{0.23}$ & & & & & 2 & 3.53 & 1.95 & 0.18 & 1 & & .039 & 0.98 & 34.9 & \begin{tabular}{|l|}
18.8 \\
\end{tabular} & & 347 \\
\hline & & & .044 & \begin{tabular}{|l|l|}
0.10 \\
\end{tabular} & .67 & 9 & 60 & .03 & & 74 & \begin{tabular}{|l|}
0.24 \\
\end{tabular} & & 21.8 & & 2.17 & 20. & 3.86 & 2.45 & 0.17 & 1. & 07 & .042 & 1.02 & 30.7 & 22.1 & 2.33 & 665 \\
\hline & & & 0.012 & 0.14 & 37 & & 40 & $\overline{99}$ & & 68 & & & & & & & & 0.35 & & & & .035 & & 3.8 & 15.0 & & 28 \\
\hline & & & .005 & \begin{tabular}{|l|}
0.09 \\
\end{tabular} & 12 & 7.7 & 460 & .75 & & 2.07 & 0.20 & 37.9 & $\overline{31.0}$ & $\overline{910}$ & 1.84 & 27. & 3.71 & 0.35 & 0.13 & 1. & & .034 & 0.63 & 19.0 & 14.8 & 3.42 & 550 \\
\hline & & & 033 & 12 & & & & & & & & & & & & $\angle 8$ & & 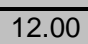 & 0.1 & & & 2 & & 4.9 & 18.4 & 2. & 714 \\
\hline & & & 0.007 & 0.14 & & & 60 & & & 28 & & & & 23 & & & & & & & & .040 & & & & & 2110 \\
\hline & & & 0.015 & \begin{tabular}{|l|l|}
0.17 \\
\end{tabular} & \begin{tabular}{|l|l|}
6.12 \\
\end{tabular} & & 810 & 1.50 & & 1.42 & \begin{tabular}{|l|l|}
0.54 \\
\end{tabular} & 69.5 & 12.2 & 7 & 35 & 29. & 3.20 & 3.70 & 0.15 & 1. & & .044 & 1.24 & 35.9 & 23.9 & 0.85 & 881 \\
\hline & & & .006 & \begin{tabular}{|l|l|}
0.14 \\
\end{tabular} & & & & & & 94 & \begin{tabular}{|l|}
0.34 \\
\end{tabular} & & & & & 25. & $3.0 \varepsilon$ & 3.75 & 0.1 & & & .042 & 1. & 31.8 & 22.4 & 1. & 522 \\
\hline & & & 005 & 13 & & & 960 & & & & 0.35 & & & & & & & & & & & & & 30 & \begin{tabular}{|l|}
21.4 \\
\end{tabular} & & $\overline{00}$ \\
\hline & & & .005 & 17 & & & 92 & & & .60 & 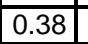 & & & & & 23 & & 85 & 0. & & & .043 & 1. & 30.3 & 21.5 & 0.9 & 497 \\
\hline & & & 023 & & & & 100 & & & & & & & & & 26 & & 2.70 & & & & 045 & 1. & 3.0 & 20.6 & J. & 730 \\
\hline & & & .005 & \begin{tabular}{|l|l|}
0.19 \\
\end{tabular} & 5.88 & 13.4 & 1020 & 1.38 & & 1.38 & 0.51 & & 18. & & & 38. & 3.92 & 4.40 & 0 & 1. & & .050 & 1. & 31.2 & \begin{tabular}{|l|}
21.9 \\
\end{tabular} & 0. & 960 \\
\hline & & & 006 & & & 7.9 & 840 & & & & 0.48 & & & & & 21. & 3. & 3.10 & & & & .045 & & 31.9 & \begin{tabular}{|l|}
19.8 \\
\end{tabular} & 0. & 1340 \\
\hline & & & .005 & & & & & & & 1. & & & & & & & & & & & & & & & 6 & & 1675 \\
\hline & & & 005 & & & & & & & & & & & & & & & & & & & & & & 5 & & 476 \\
\hline & & & .005 & 11 & & 7.0 & & & & 1.52 & & & & & & & & 3.85 & 0. & & & .045 & & 35.0 & 20.2 & 0. & $\overline{942}$ \\
\hline & & & & & & & & & & 1.64 & 0.30 & & 9.5 & & & & & & & & & & & & \begin{tabular}{|l|}
7.4 \\
\end{tabular} & 0. & 466 \\
\hline & & & 005 & & & & & & & & & & & & & & & & & & & $\overline{0}$ & & 31.5 & 20.6 & 0. & 1035 \\
\hline & & & .005 & 0.13 & 5.92 & 8 & & & & 1.49 & 0.3 & & & & & & & 3.80 & & & & .045 & & 35.4 & 20.9 & \begin{tabular}{|l|l}
$0 . \varepsilon$ \\
\end{tabular} & 617 \\
\hline & & & & & & & & & & & & & & & & & & & & & & & & & 18.7 & & 1035 \\
\hline & & & .005 & 0.10 & & & & & & 1. & & & & & & & & 3.40 & & & & .044 & & 31.6 & \begin{tabular}{|l|}
18.6 \\
\end{tabular} & 0. & 953 \\
\hline & & & .005 & \begin{tabular}{|l|}
0.15 \\
\end{tabular} & 5.40 & 9 & & $1.2 C$ & & 1.70 & \begin{tabular}{|l|}
0.84 \\
\end{tabular} & & & & & 25. & 3.1 & 2.65 & 0.17 & 1.4 & 21 & .040 & 1.12 & 27.9 & \begin{tabular}{|l|}
17.8 \\
\end{tabular} & \begin{tabular}{|l|l}
0.81 \\
\end{tabular} & 980 \\
\hline & & & & & & & & & & & & & & & & & & & & & & & & 22.4 & \begin{tabular}{|l|}
17.4 \\
\end{tabular} & & 645 \\
\hline & 2759 & & 0.005 & 0.11 & 5.82 & 6.5 & 850 & 1.22 & & 1.72 & 0. & & 11.1 & & 2. & 21.3 & 2.98 & 3.30 & 17 & 1 & 20 & .041 & $\begin{array}{l}1.20 \\
\end{array}$ & 31.9 & \begin{tabular}{|l|}
18.2 \\
\end{tabular} & \begin{tabular}{|l|} 
\\
\end{tabular} & 535 \\
\hline & & & 0.006 & 0.11 & 5.75 & & 840 & & & 1. & 0.4 & & & & & & & & & & & & & 30.4 & \begin{tabular}{|l|}
17.2 \\
\end{tabular} & 0.90 & 464 \\
\hline & & & & & & & & & & & & & & & & & & & & & & & & & \begin{tabular}{|l|}
19.4 \\
\end{tabular} & & 2370 \\
\hline & & 7272582 & .005 & 0.11 & 6.01 & 5.9 & 900 & 1.19 & $\overline{0 .}$ & 1.77 & 0.29 & 74 & 10.4 & 91 & 2.2 & 21. & 2.84 & 13.80 & & 1.9 & 0.31 & .043 & 1.22 & 38.4 & 19.4 & \begin{tabular}{|l|}
0.95 \\
\end{tabular} & 501 \\
\hline & & & & & & 6.5 & 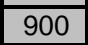 & & & & & & & & & & & & & & & 0.075 & & 30.7 & 20.4 & 0.94 & 462 \\
\hline
\end{tabular}


Table 1. Location and New Trace Element Geochemical Results for 902 Stream-Sediment Samples collected in the Livengood Area, Livengood Quadrangle, Alaska IS = Insufficient Sample for Analysis

\begin{tabular}{|c|c|c|c|c|c|c|c|c|c|c|c|c|c|c|c|c|c|c|c|c|c|c|c|c|c|c|}
\hline AMPLE & $\begin{array}{l}\text { Mo } \\
\text { ppm }\end{array}$ & \begin{tabular}{l|}
$\mathrm{Na}$ \\
$\%$
\end{tabular} & $\begin{array}{l}\mathrm{Nb} \\
\mathrm{ppm}\end{array}$ & $\begin{array}{c}\mathrm{Ni} \\
\mathrm{ppm}\end{array}$ & $\begin{array}{c}\mathrm{P} \\
\mathrm{ppm}\end{array}$ & $\begin{array}{l}\mathrm{Pb} \\
\mathrm{ppm}\end{array}$ & $\begin{array}{l}\mathrm{Rb} \\
\mathrm{ppm}\end{array}$ & $\begin{array}{c}\mathrm{Re} \\
\mathrm{ppm}\end{array}$ & $\begin{array}{l}\mathrm{S} \\
\%\end{array}$ & $\begin{array}{l}\text { Sb } \\
\mathrm{ppm}\end{array}$ & $\begin{array}{c}\text { Se } \\
\text { ppm }\end{array}$ & $\begin{array}{l}S^{\text {Sn }} \\
\mathrm{ppm}\end{array}$ & $\begin{array}{c}\mathrm{Sr} \\
\mathrm{ppm}\end{array}$ & $\begin{array}{l}\mathrm{Ta}^{*} \\
\mathrm{ppm}\end{array}$ & $\begin{array}{c}\text { Te } \\
\text { ppm }\end{array}$ & $\begin{array}{c}\text { Th } \\
\text { ppm }\end{array}$ & $\begin{array}{l}\mathrm{Ti}^{*} \\
\%\end{array}$ & \begin{tabular}{c|}
$\mathrm{TI}$ \\
$\mathrm{ppm}$
\end{tabular} & $\begin{array}{c}U \\
\mathrm{ppm}\end{array}$ & $\begin{array}{c}\mathrm{V} \\
\mathrm{ppm}\end{array}$ & $\begin{array}{l}W^{*} \\
\text { ppm }\end{array}$ & $\begin{array}{c}\mathrm{Y} \\
\mathrm{ppm}\end{array}$ & $\begin{array}{l}\mathrm{Zn} \\
\mathrm{ppm}\end{array}$ & $\begin{array}{l}\mathrm{Zr}^{*} \\
\mathrm{ppm}\end{array}$ & $\begin{array}{c}\text { Pulp Wt } \\
\text { grams }\end{array}$ & $\begin{array}{c}\text { Lab } \\
\text { Report }\end{array}$ \\
\hline 1128 & 1.16 & 1.16 & 10.2 & 25.2 & 660 & 12.1 & 57.8 & $<0.002$ & 0.02 & 1.08 & 1 & 1.60 & 202.0 & 0.73 & $<0.05$ & 8.5 & 0.401 & 0.38 & 2.4 & 117 & 1.1 & 16.0 & 70 & 67.0 & 28 & FA04030731 \\
\hline 129 & 1.80 & 93 & 10.0 & 30.7 & 740 & 14.7 & 51.2 & 0.002 & 0.03 & 1.40 & 2 & 1.90 & 75.0 & 0.68 & 0.08 & 8.6 & 382 & 0.45 & 2.9 & 125 & 1.2 & 18.5 & 76 & 73.7 & 21 & FA04030731 \\
\hline 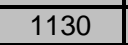 & 0.90 & 30 & 9.8 & 45.4 & 1260 & 8.5 & 47.4 & $<0.002$ & 0.09 & 0.82 & 2 & 1.50 & 130.0 & \begin{tabular}{|l|l|}
0.67 \\
\end{tabular} & 0.05 & 5.9 & .377 & 0.26 & 1.9 & 89 & 0.8 & 19.1 & 88 & 63.6 & & A04030731 \\
\hline & 14 & & 12.2 & 40.4 & 880 & 10.3 & 6.8 & $<0.002$ & 0.10 & 21 & & 2.00 & 127.0 & 0.82 & 0.08 & 6.2 & .384 & 0.34 & 3.5 & 127 & & 29.3 & 108 & 92.3 & & \\
\hline 33 & 2.38 & .70 & 17.2 & 49.5 & 1100 & 13.6 & 3.1 & $<0.002$ & 0.05 & 1.33 & 2 & 2.00 & 146.5 & 1.15 & 0.12 & 7.5 & 0.590 & 0.39 & 3.9 & 164 & 1.2 & 28.0 & 107 & 117.5 & 22 & A04030731 \\
\hline & 2.47 & 60 & 16.6 & 45.6 & 1120 & 13.7 & 2.2 & $<0.002$ & 0.05 & 1.26 & 2 & 2.20 & 127.0 & 1.13 & 0.09 & 7.1 & 0.592 & 0.44 & 3.6 & 169 & 1.1 & 27.3 & 120 & 108.0 & & FA04030731 \\
\hline 135 & 2.52 & 73 & 14.2 & 49.4 & 1180 & 14.9 & 74.9 & $<0.002$ & 0.05 & 1.49 & 2 & 3.10 & 153.0 & 1.12 & 0.10 & 7.0 & 0.497 & 0.43 & 3.6 & 165 & 1.3 & 24.7 & 101 & 104.5 & & FA04030731 \\
\hline & 2.99 & $\begin{array}{ll}0.64 \\
\end{array}$ & 18.7 & 48.0 & 1260 & 15.6 & 66.1 & 0.002 & 0.05 & 1.48 & 2 & 2.40 & 138.5 & \begin{tabular}{|l|l|}
1.41 \\
\end{tabular} & 0.11 & 7.1 & 0.640 & 0.43 & 3.9 & 181 & 1.2 & 29.3 & 127 & 131.0 & & FA04030731 \\
\hline & 1.33 & 21 & 12.6 & 36.4 & 910 & 15.0 & 63.3 & $<0.002$ & 0.03 & 1.44 & & 1.80 & 224.0 & 1.02 & 0.06 & $\overline{9.4}$ & 0.469 & 0.39 & 2.7 & 131 & & 21.8 & 88 & 91.5 & & FA04030731 \\
\hline & 0.43 & 0.84 & 4.4 & 539.0 & 600 & 8.3 & 27.7 & 0.002 & 0.05 & 1.70 & 1 & 1.50 & 125.5 & 0.40 & 0.05 & 3.7 & .269 & 0.15 & 1.0 & 116 & 0.6 & 9.6 & 96 & 34.3 & & A04030731 \\
\hline & 0.31 & 1.05 & 7.6 & 329.0 & 540 & 7.8 & 39.1 & $<0.002$ & 0.03 & 1.08 & 1 & 1.30 & 173.5 & 0.63 & $<0.05$ & 6.8 & 0.380 & 0.23 & 1.7 & 110 & 0.9 & 14.3 & 78 & 59.0 & 2 & FA04030731 \\
\hline & & 1.23 & 5.1 & 323.0 & 510 & 6.8 & 29.1 & $<0.002$ & 0.03 & 0.81 & & 1.00 & 186.5 & 0.54 & $<0.05$ & 4.0 & 0.378 & 0.16 & 1.1 & 153 & & 13.0 & 100 & & & \\
\hline & 0.30 & 1.02 & 7.2 & 385.0 & 480 & 7.1 & 34.7 & $<0.002$ & 0.02 & 1.04 & 1 & 1.10 & 164.5 & 0.59 & $<0.05$ & 5.5 & 0.373 & 0.19 & 1.4 & 120 & 0.9 & 13.8 & 83 & 55.7 & 5 & FA04030731 \\
\hline & 0.34 & 0.92 & 6.7 & 352.0 & 550 & 7.7 & 36.5 & $<0.002$ & 0.03 & 1.24 & 1 & 1.30 & 140.5 & 0.53 & $<0.05$ & 4.9 & 0.374 & 0.21 & 1.4 & 123 & 0.7 & 12.8 & 96 & 50.8 & 21 & FA04030731 \\
\hline & 59 & 1.14 & 8.9 & 164.0 & 690 & 10.2 & 48.8 & $<0.002$ & 0.03 & 1.04 & & 1.40 & 194.5 & \begin{tabular}{|l|}
0.72 \\
\end{tabular} & $<0.05$ & 7.2 & 0.392 & 0.30 & 1.8 & 114 & & 15.4 & & 59.0 & & 731 \\
\hline & 0.53 & 1.10 & 8.2 & 206.0 & 650 & 9.5 & 44.6 & $<0.002$ & 0.02 & 1.02 & 1 & 1.40 & 177.0 & 0.66 & $<0.05$ & 7.3 & 0.396 & 0.29 & 1.8 & 118 & 5.5 & 14.6 & 79 & 60.2 & 2 & $\overline{\text { FA0403 }}$ \\
\hline & 0.76 & 1.15 & 8.7 & 180.0 & 670 & 9.2 & 46.3 & $<0.002$ & 0.02 & 1.28 & & 1.40 & 157.0 & 0.65 & $<0.05$ & 6.0 & 0.449 & 0.25 & 1.7 & 135 & 2.7 & 15.2 & & 66.3 & & FA0403 \\
\hline & 0.41 & 0.89 & 6.7 & 327.0 & 640 & 8.1 & 43.8 & $<0.002$ & 0.16 & 1.44 & 4 & 1.30 & 210.0 & 0.56 & $<0.05$ & 5.5 & 0.283 & 0.28 & 1.8 & 94 & 0.8 & 20.5 & & & & $\overline{F A 0403}$ \\
\hline & 0.30 & 1.01 & 5.4 & 390.0 & 430 & 6.6 & 29.5 & $<0.002$ & 0.10 & 1.42 & 2 & 1.10 & 184.0 & 0.44 & $<0.05$ & 4.5 & 0.369 & 0.20 & 1.2 & 116 & 0.7 & 12.5 & $\overline{82}$ & 42.2 & 2 & FA04030731 \\
\hline & $\overline{45}$ & 1.10 & 7.1 & 277.0 & 470 & 9.4 & 42.7 & $<0.002$ & 0.07 & 1.60 & & 1.30 & 174.0 & 0.56 & $<0.05$ & 5.8 & .363 & 0.28 & 1.6 & 114 & $\overline{0.8}$ & 14.5 & & 54.9 & & FA0403 \\
\hline & 61 & 0.90 & 7.5 & 264.0 & 810 & 11.2 & 56.2 & $<0.002$ & 0.09 & 1.55 & & 2.60 & 164.0 & 0.58 & $<0.05$ & 6.3 & 0.315 & 0.35 & 1.5 & 110 & 0. & 14.0 & 8 & & & \\
\hline & 1.01 & 1.13 & 8.6 & 33.3 & 810 & 13.8 & 61.6 & $<0.002$ & 0.04 & 1.12 & & 1.60 & 266.0 & \begin{tabular}{|l|}
0.69 \\
\end{tabular} & $<0.05$ & 7.5 & 0.357 & 0.38 & 2.4 & 110 & 1.0 & 18.8 & $11 \varepsilon^{2}$ & 70.0 & & FA04030731 \\
\hline & & 1.25 & 10.4 & 32.4 & 840 & 12.4 & 59.5 & .002 & 0.03 & 1.20 & & .10 & 238.0 & 0.81 & $<0.05$ & 8.0 & .390 & 0.36 & 2.0 & 119 & 1.0 & 17.8 & & 57. & & 31 \\
\hline & 83 & 1.30 & 9.5 & 31.7 & 840 & 12.1 & 57.7 & $<0.002$ & 0.03 & 1.12 & 1 & 1.60 & 248.0 & 0.74 & $<0.05$ & 7.6 & 0.372 & 0.36 & 2.0 & 119 & 0.9 & 17.0 & & 64.7 & & $\mathrm{FAO2}$ \\
\hline & 0.75 & 1.25 & 9.6 & 31.0 & 790 & 12.8 & 59.0 & $<0.002$ & 0.03 & 1.12 & & 1.60 & 236.0 & 0.75 & $<0.05$ & 7.4 & 0.376 & 0.37 & 2.1 & 116 & 1.0 & 17.0 & 84 & 64.4 & & FA04 \\
\hline & 1.34 & 0.97 & 8.4 & 39.5 & 1030 & 14.6 & 58.8 & 0.002 & 0.07 & 1.40 & & 1.50 & 215.0 & 0.67 & 0.06 & 7.5 & 0.324 & 0.38 & 2.1 & & 1.1 & 21.1 & 1 & 58.9 & & FA0403 \\
\hline & 2.06 & 0.97 & 11.6 & 42.9 & 1200 & 13.6 & 62.7 & 0.002 & 0.04 & 1.38 & & 1.80 & 200.0 & 0.91 & 0.07 & 7.4 & .443 & 0.42 & 2.8 & 135 & 1.1 & 20.5 & 96 & 83.2 & & FA040 \\
\hline 61 & 0.90 & \begin{tabular}{|l|l|}
1.21 \\
\end{tabular} & 9.7 & 30.7 & 870 & 12.4 & 56.4 & 0.002 & 0.04 & 1.05 & & 1.80 & 231.0 & 0.75 & $<0.05$ & 7.7 & 0.385 & 0.35 & 2.2 & 113 & 1.0 & 17.4 & & 68.3 & & FA04030731 \\
\hline & & 1.17 & 10.8 & 35.3 & 970 & 13.2 & 57.0 & $<0.002$ & 0.03 & 1.26 & & & 222.0 & 0.83 & & 8.2 & & 0.37 & 2. & & & 18. & & 79 & & \\
\hline & 0.87 & 1.29 & 10.6 & 27.9 & 830 & 12.0 & 54.5 & $<0.002$ & 0.02 & 1.10 & & 1.60 & 230.0 & 0.81 & $<0.05$ & 8.5 & 0.399 & 0.33 & 2.2 & 112 & 1. & 18. & & 71. & & FA040 \\
\hline & 0.97 & 1.28 & 10.3 & 27.6 & 820 & 12.0 & 55.2 & $<0.002$ & 0.03 & 1.00 & & 1.60 & 228.0 & 0.78 & 0.05 & 8.3 & 0.444 & 0.32 & 2.4 & 1 & 1.3 & 18.8 & & 82.2 & & FA04030731 \\
\hline & & 1.26 & 9.6 & 26.7 & 790 & 11.6 & & .002 & 0.02 & 1.06 & & & 226.0 & 0.76 & $<0.05$ & 7.8 & & 0.34 & 2.1 & 107 & & 16. & & 65.8 & & \\
\hline 68 & 0.99 & 1.18 & 9.6 & 28.9 & 820 & 11.7 & 54.3 & $<0.002$ & 0.04 & 1.00 & & 1.50 & 213.0 & 0.75 & 0.05 & 7.3 & 0.413 & 0.33 & 2.2 & 114 & 0.9 & 18.6 & 88 & 78.1 & & FA04030731 \\
\hline & 1.05 & 1.16 & 11.0 & 29.2 & 790 & 13.1 & 57.0 & $<0.002$ & 0.02 & 1.12 & & 1.80 & 212.0 & 0.87 & $<0.05$ & 8.9 & 0.431 & 0.36 & 2.4 & 122 & 1.3 & 18.6 & & 74.9 & & FA0403C \\
\hline & & & \begin{tabular}{|l|}
9.7 \\
\end{tabular} & 28.9 & 810 & 12.4 & 54.0 & $<0.002$ & 0.03 & 1.00 & & & & 0.76 & & 7.7 & & & 2.3 & 115 & & & & & & FA0403 \\
\hline 72 & 1.06 & 1.20 & 9.5 & 27.5 & 780 & 11.4 & 53.5 & $<0.002$ & 0.03 & 0.96 & & 1.50 & 212.0 & 0.74 & $<0.05$ & 7.5 & 0.407 & 0.33 & 2.2 & 114 & 1.0 & 18.1 & 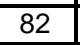 & 71.4 & & FA04030731 \\
\hline 1174 & 0.96 & 0.95 & 9.2 & 31.6 & 900 & 12.2 & 55.3 & $<0.002$ & 0.05 & 1.54 & & 1.50 & 175.5 & 0.74 & 0.05 & 7.3 & 0.364 & 0.38 & 2.2 & 114 & 0.9 & \begin{tabular}{|l|}
16.6 \\
\end{tabular} & 95 & 61.5 & & FA04030731 \\
\hline & & & 8.4 & 37.3 & & 10.0 & 53.0 & & 0.07 & & & & 126.0 & 0.65 & 0.09 & 5.0 & & & 2.5 & & 1.1 & & & 66.4 & & FA04030731 \\
\hline 1176 & 0.69 & 1.24 & 10.6 & 28.8 & 790 & 11.2 & 55.0 & $<0.002$ & 0.03 & 1.12 & 1 & 1.60 & 219.0 & 0.82 & $<0.05$ & 7.7 & 0.415 & 0.33 & 2.1 & 118 & 1.1 & 17.2 & 77 & 64.5 & & FA04030731 \\
\hline & 0.52 & 1.2 & 9.9 & 28.3 & 820 & 10.9 & 53.1 & $<0.002$ & 0.02 & 1.19 & & 1.50 & 223.0 & 0.78 & $<0.05$ & 7.6 & 0.406 & 0.35 & 2.3 & 118 & 0 . & 16.2 & 83 & 64.6 & & FA04030731 \\
\hline & 0.91 & 1.0 & 8.6 & 32.6 & 1150 & 11.3 & 58. & & 0.07 & 1.3 & & & & 0.54 & & 6.7 & & & 2.0 & 110 & & & 9 & 63.0 & & FA04030731 \\
\hline 1179 & 0.63 & 1.35 & 11.0 & 28.5 & 810 & 11.8 & 54.7 & $<0.002$ & 0.02 & 1.17 & 1 & 1.50 & 237.0 & 0.72 & $<0.05$ & 9.8 & 0.472 & 0.32 & 2.5 & 126 & 1.4 & 19.1 & 74 & 79.5 & 65 & FA04030731 \\
\hline 1180 & 0.65 & 1.35 & 10.6 & 27.0 & 780 & 12.2 & 55.8 & $<0.002$ & 0.02 & 1.12 & & 1.60 & 232.0 & \begin{tabular}{|c|}
0.68 \\
\end{tabular} & $<0.05$ & 7.8 & 0.418 & 0.34 & 2.2 & 120 & 1.2 & 17.0 & 76 & 69.0 & $\overline{52}$ & FA04030731 \\
\hline
\end{tabular}


Table 1. Location and New Trace Element Geochemical Results for 902 Stream-Sediment Samples collected in the Livengood Area, Livengood Quadrangle, Alaska IS = Insufficient Sample for Analysis

\begin{tabular}{|c|c|c|c|c|c|c|c|c|c|c|c|c|c|c|c|c|c|c|c|c|c|c|c|c|c|c|c|}
\hline MPLE & TM E & TM N & $\begin{array}{c}\mathrm{Au} \\
\mathrm{ppm}\end{array}$ & \begin{tabular}{|c|}
$\mathrm{Ag}$ \\
$\mathrm{ppm}$
\end{tabular} & $\begin{array}{l}\text { Al } \\
\%\end{array}$ & $\begin{array}{c}\text { As } \\
\text { ppm }\end{array}$ & $\begin{array}{l}\mathrm{Ba}^{*} \\
\mathrm{ppm}\end{array}$ & \begin{tabular}{|c|}
$\mathrm{Be}$ \\
$\mathrm{ppm}$
\end{tabular} & $\begin{array}{c}\mathrm{Bi} \\
\mathrm{ppm}\end{array}$ & $\begin{array}{c}\mathrm{Ca} \\
\%\end{array}$ & \begin{tabular}{|c|}
$\mathrm{Cd}$ \\
$\mathrm{ppm}$
\end{tabular} & $\begin{array}{l}\mathrm{Ce} \\
\mathrm{ppm}\end{array}$ & $\begin{array}{l}\text { Co } \\
\text { ppm }\end{array}$ & $\begin{array}{l}\mathrm{Cr}^{\star} \\
\mathrm{ppm}\end{array}$ & $\begin{array}{l}\text { Cs } \\
\text { ppm }\end{array}$ & $\begin{array}{c}\mathrm{Cu} \\
\mathrm{ppm}\end{array}$ & $\begin{array}{c}\mathrm{Fe} \\
\% \\
\end{array}$ & $\begin{array}{c}\mathrm{Ga} \\
\mathrm{ppm}\end{array}$ & $\begin{array}{c}\mathrm{Ge} \\
\mathrm{ppm}\end{array}$ & $\begin{array}{c}\mathrm{Hf} \\
\mathrm{ppm}\end{array}$ & $\begin{array}{l}\mathrm{Hg} \\
\mathrm{ppm}\end{array}$ & $\begin{array}{c}\text { In } \\
\mathrm{ppm}\end{array}$ & $\begin{array}{l}\mathrm{K} \\
\% \\
\end{array}$ & $\begin{array}{c}\mathrm{La} \\
\mathrm{ppm}\end{array}$ & \begin{tabular}{|c|}
$\mathrm{Li}$ \\
$\mathrm{ppm}$
\end{tabular} & $\begin{array}{c}\mathrm{Mg} \\
\% \\
\end{array}$ & $\begin{array}{l}\mathrm{Mn} \\
\mathrm{ppm}\end{array}$ \\
\hline 181 & 6645 & & $<0.005$ & 0.10 & & 8.4 & 770 & 1.18 & 0.15 & & & & 95 & 90 & 60 & 15.3 & & & 0.12 & 1.6 & 0.13 & 0.045 & 1.22 & 37.9 & \begin{tabular}{|l|}
20.7 \\
\end{tabular} & 0.92 & 391 \\
\hline & & & & & & & 790 & & & & & & & & & 6. & 86 & & 0.12 & 1. & & & \begin{tabular}{|l|}
1.18 \\
\end{tabular} & 33.0 & 22.1 & 0.87 & 881 \\
\hline & & & 009 & 13 & & & 890 & & 15 & & & & & & & 22.5 & & 4.65 & 0.12 & 1. & 27 & .048 & 1.30 & 2.6 & 27.8 & & 446 \\
\hline & & & & & & & 810 & & & & & & & & & & & & & & & & & 35.0 & & & 42 \\
\hline & & & .008 & 0.16 & $\overline{93}$ & 1.4 & 880 & 1.30 & 17 & 67 & 0.42 & $\overline{5.7}$ & 3.6 & & .47 & 28.3 & .38 & 3.75 & 0.14 & 1. & 11 & .046 & 1.24 & 33.7 & \begin{tabular}{|l|}
21.2 \\
\end{tabular} & 095 & 733 \\
\hline & & & .005 & \begin{tabular}{|l|l|}
0.14 \\
\end{tabular} & 47 & 9.6 & 1050 & 1.28 & 13 & 49 & $\begin{array}{ll}0.22 \\
\end{array}$ & $\overline{1.5}$ & 9.9 & & .12 & 22.5 & 2.75 & 13.20 & 0.13 & 1.6 & 0.20 & .042 & 1.06 & 37.2 & \begin{tabular}{|l|}
20.1 \\
\end{tabular} & 0.77 & 470 \\
\hline & & & 005 & \begin{tabular}{|l|}
0.13 \\
\end{tabular} & & & \begin{tabular}{|l|}
1170 \\
\end{tabular} & 1.13 & & & \begin{tabular}{|l|}
0.32 \\
\end{tabular} & & & & & 251 & 307 & & & & & & & 292 & & & 393 \\
\hline & & & 0.007 & \begin{tabular}{|l|}
0.13 \\
\end{tabular} & 49 & & 980 & 1.22 & $\overline{14}$ & 24 & & 5.5 & 22.8 & 9 & .86 & 22.5 & 3.60 & 13.50 & 0.12 & 1. & .26 & \begin{tabular}{|l|l|}
0.040 \\
\end{tabular} & 1.03 & 27.5 & \begin{tabular}{|l|}
20.9 \\
\end{tabular} & 0.77 & 1560 \\
\hline & & & 005 & 20 & & & 1560 & 1.61 & $\overline{18}$ & 02 & .59 & 9.3 & 0.0 & 0 & 4.14 & 46.3 & 3.62 & 16.55 & 0.13 & 1.8 & 0.36 & 0.056 & 1.24 & 30.2 & 25.7 & 0.18 & 480 \\
\hline & & & .005 & 13 & & 6.5 & 900 & \begin{tabular}{|l|}
1.18 \\
\end{tabular} & 13 & 14 & \begin{tabular}{|l|}
0.30 \\
\end{tabular} & $\overline{41}$ & $\overline{1.0}$ & & 2.4 & 25.4 & 2.65 & 12.10 & 0.12 & & 0.24 & & \begin{tabular}{|l|}
0.97 \\
\end{tabular} & 26.9 & \begin{tabular}{|l|}
19.4 \\
\end{tabular} & & 006 \\
\hline & & & .005 & 0.14 & 5.56 & 7.0 & 980 & 1.35 & 14 & 1.22 & \begin{tabular}{|l|}
0.39 \\
\end{tabular} & $\overline{1.2}$ & s. & & 2.66 & 24.6 & 2.99 & 13.60 & 0.12 & 1. & 18 & 0.043 & 1.08 & 31.9 & \begin{tabular}{|l|}
24.5 \\
\end{tabular} & 0.84 & 662 \\
\hline & & & .005 & 13 & & & 890 & 19 & & 34 & & & & & & 18. & 2.86 & 12.60 & 0.12 & & .15 & 0.040 & \begin{tabular}{|l|}
1.07 \\
\end{tabular} & 33.9 & \begin{tabular}{|l|l|}
18.7 \\
\end{tabular} & 0.0 & 338 \\
\hline & & & 009 & 11 & & & 840 & .12 & & 40 & & & & & $\sqrt{2}$ & 15 & 75 & 3.05 & & & & .043 & & 87.0 & 39.9 & & 91 \\
\hline & & & .005 & 0.10 & 5.59 & 7.7 & 980 & 1.13 & 13 & .34 & \begin{tabular}{|l|}
0.44 \\
\end{tabular} & 63.8 & 15.4 & & 2.56 & 21.2 & 3.04 & 13.65 & 0.13 & 1.7 & 0.30 & .043 & 1.14 & 32.1 & \begin{tabular}{|l|l|}
19.8 \\
\end{tabular} & 0.88 & 01 \\
\hline & & & .005 & & & & 910 & & & & & & & & & $\angle U$. & 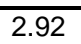 & 13.20 & 0.13 & & 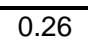 & .045 & & 34.0 & \begin{tabular}{|l|l|}
18.0 \\
\end{tabular} & & 996 \\
\hline & & & .006 & 0.15 & & & 760 & 1.09 & & & & & 9.3 & & 2.7 & 24. & 25 & 2.95 & & 1. & 33 & 039 & & 25.1 & \begin{tabular}{|l|}
18.2 \\
\end{tabular} & & 84 \\
\hline & & & .005 & \begin{tabular}{|l|l|}
0.14 \\
\end{tabular} & 10 & & 750 & 1.13 & & 82 & 24 & & & & 8 & 27. & & 2.80 & 0.09 & 1. & 3 & .042 & 1.08 & 88.2 & 18.8 & .6 & 992 \\
\hline & & & $\overline{005}$ & 18 & & & 880 & & & & & & & & & & & 0 & & & & .048 & & & 21.5 & & 550 \\
\hline & & & 005 & \begin{tabular}{|l|l|}
0.87 \\
\end{tabular} & 41 & 20.8 & 940 & 1.49 & 19 & 1.04 & 1.99 & 77.5 & & & 4.0 & 59. & 3.75 & 6.80 & 0.19 & 2. & & & 1.4 & 44.1 & 35.2 & 0.8 & 660 \\
\hline & & & .0 & 56 & & & & & & & & & & & & 44 & & 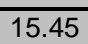 & $\bar{x}$ & & & & & 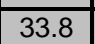 & 31.0 & & 761 \\
\hline & & & .005 & 0.26 & & & 1000 & & & & & & & $\bar{\Omega}$ & & & 3.07 & & & & & & & & 36.3 & & 141 \\
\hline & & & .005 & 0.20 & 87 & .0 & 940 & \begin{tabular}{|l|}
1.30 \\
\end{tabular} & 0.14 & 1.16 & \begin{tabular}{|l|}
0.42 \\
\end{tabular} & 911 & 11. & & 3.4 & 21.9 & 3.06 & 14.95 & 0.15 & 1. & 0.43 & .051 & 1.16 & 44.6 & \begin{tabular}{|l|}
36.5 \\
\end{tabular} & 0.7 & 557 \\
\hline & & & 0.007 & 11 & & & 780 & & & & \begin{tabular}{|l|}
0.30 \\
\end{tabular} & & & & & 17. & 2.8 & 3.75 & 0.12 & & .3 & .041 & 1. & 29.9 & 35.1 & & 41 \\
\hline & & & 005 & & & 12 & 840 & & & & & & & & & & & & & & & & & 35.7 & & & 94 \\
\hline & & & $\overline{008}$ & 12 & & & 30 & & & & & & & & & 16. & & 4.00 & 0.1 & & 0.3 & .049 & 1. & 47.4 & \begin{tabular}{|l|}
24.6 \\
\end{tabular} & 0.8 & 687 \\
\hline & & & & & & & & & & & & & & & & & & 3. & & & & .044 & & 0.4 & 9.4 & & 490 \\
\hline & & & 006 & 0.10 & & $\overline{9}$ & 770 & 1.12 & & & & & & & & & 3.01 & 4.65 & 0.14 & & & 046 & 1. & 36.0 & \begin{tabular}{|l|}
30.0 \\
\end{tabular} & & 514 \\
\hline & & & .00 & 12 & & & 740 & & & & & & & & & & & & & & & 46 & & 43.6 & 26.7 & & 516 \\
\hline & & & & & & & 830 & & & & & & & & & & & & & & & & & & \begin{tabular}{|l|}
31.3 \\
\end{tabular} & & 590 \\
\hline & & & & & & & 88 & & & & & & & & & & & & & & & & & & 32.1 & & 2020 \\
\hline & & & 010 & .11 & & 9.7 & 860 & & & & & & & & & 24. & 3.9 & 1.30 & 0.16 & & 24 & .065 & & 41.2 & 54.6 & & 967 \\
\hline & & & & & & 10.5 & & & & & & & & & & & & & & & & & & 46.2 & 72.1 & & 678 \\
\hline & & & & & & & & & & & & & & & & & & & & & & & & 43.9 & 60.3 & & 746 \\
\hline & & & .005 & \begin{tabular}{|l|}
0.58 \\
\end{tabular} & & 11.3 & 1640 & & & 1.54 & 0.70 & & & & & 33. & & 16.75 & & & & .058 & & 35.4 & \begin{tabular}{|l|l|}
44.3 \\
\end{tabular} & 0.8 & 1315 \\
\hline & & & & 0.43 & & & & & & & & & & & & & & & & & & & & 34.5 & \begin{tabular}{|l|}
35.4 \\
\end{tabular} & & 1250 \\
\hline & & & $\overline{010}$ & & & & & & & & & & & & & & & 4.80 & & & & 051 & & 35.7 & 30.1 & & 743 \\
\hline & & & .005 & \begin{tabular}{|l|}
0.30 \\
\end{tabular} & 6.30 & 12.2 & 2450 & 1.58 & & 1.00 & 0.76 & & & & & & 3.2 & 6.15 & 0.17 & & & 054 & | 1.68 & 37.0 & \begin{tabular}{|l|}
40.2 \\
\end{tabular} & 0. & 838 \\
\hline & & & & & & & & & & & & & & & & & & & & & & & & & \begin{tabular}{|l|l|}
40.0 \\
\end{tabular} & & 754 \\
\hline & & & 0.007 & 0.26 & & 9 & & & & 1. & & & & & & & & 6.10 & & & 0.4 & 055 & 1.52 & 43.2 & \begin{tabular}{|l|}
36.3 \\
\end{tabular} & & 861 \\
\hline & & & 0.006 & 0.25 & \begin{tabular}{|l|l}
6.06 \\
\end{tabular} & 7.7 & 1460 & 1. & & 1. & \begin{tabular}{|l|l|}
0.60 \\
\end{tabular} & 73 & 12 & & & 60.4 & 2. & 4.55 & 0.16 & 1. & 0.30 & .047 & 1.46 & 37.3 & \begin{tabular}{|l|}
32.2 \\
\end{tabular} & 0.84 & 771 \\
\hline & & & & & & 8.6 & & & & & & & & & & & & & & & & & & 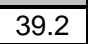 & \begin{tabular}{|l|}
35.2 \\
\end{tabular} & & 917 \\
\hline & & & $<0.005$ & 0.10 & 6.26 & 7.9 & 690 & 1.21 & 0.18 & 0.96 & 0.15 & 72 & 10. & 9 & 3.2 & 17.2 & $3 .($ & 15.30 & 0.1 & 1. & 0.13 & .048 & $\mid$\begin{tabular}{|l}
1.21 \\
\end{tabular} & 35.9 & \begin{tabular}{|l|}
26.8 \\
\end{tabular} & 0.84 & 474 \\
\hline & & & & & & 7.4 & 720 & & & & & & & & & 19.1 & & & & & & & & 34.1 & 32.0 & 0.91 & 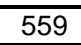 \\
\hline
\end{tabular}


Table 1. Location and New Trace Element Geochemical Results for 902 Stream-Sediment Samples collected in the Livengood Area, Livengood Quadrangle, Alaska IS = Insufficient Sample for Analysis

\begin{tabular}{|c|c|c|c|c|c|c|c|c|c|c|c|c|c|c|c|c|c|c|c|c|c|c|c|c|c|c|}
\hline AMPLE & $\begin{array}{l}\text { Mo } \\
\text { ppm }\end{array}$ & $\begin{array}{c}\mathrm{Na} \\
\% \\
\end{array}$ & \begin{tabular}{|l|}
$\mathrm{Nb}$ \\
$\mathrm{ppm}$
\end{tabular} & $\begin{array}{r}\mathrm{Ni} \\
\mathrm{ppm}\end{array}$ & $\begin{array}{c}\mathrm{P} \\
\mathrm{ppm}\end{array}$ & $\begin{array}{c}\mathrm{Pb} \\
\mathrm{ppm}\end{array}$ & $\begin{array}{l}\mathrm{Rb} \\
\mathrm{ppm}\end{array}$ & $\begin{array}{c}\mathrm{Re} \\
\mathrm{ppm}\end{array}$ & $\begin{array}{l}\mathrm{S} \\
\%\end{array}$ & $\begin{array}{c}\text { Sb } \\
\mathrm{ppm}\end{array}$ & \begin{tabular}{|c|} 
Se \\
ppm
\end{tabular} & $\begin{array}{l}S^{S n^{*}} \\
\text { ppm }\end{array}$ & $\begin{array}{c}\mathrm{Sr} \\
\mathrm{ppm}\end{array}$ & $\begin{array}{l}\mathrm{Ta}^{*} \\
\mathrm{ppm}\end{array}$ & $\begin{array}{c}\mathrm{Te} \\
\mathrm{ppm}\end{array}$ & $\begin{array}{c}\text { Th } \\
\text { ppm }\end{array}$ & $\begin{array}{l}\mathrm{Ti}^{\star} \\
\% \\
\end{array}$ & \begin{tabular}{c|}
$\mathrm{Tl}$ \\
$\mathrm{ppm}$
\end{tabular} & $\begin{array}{c}\mathrm{U} \\
\mathrm{ppm}\end{array}$ & $\begin{array}{c}\mathrm{V} \\
\mathrm{ppm}\end{array}$ & $\begin{array}{l}W^{*} \\
\mathrm{ppm}\end{array}$ & \begin{tabular}{|c|}
$\mathrm{Y}$ \\
$\mathrm{ppm}$ \\
\end{tabular} & $\begin{array}{l}\mathrm{Zn} \\
\mathrm{ppm}\end{array}$ & $\begin{array}{l}\mathrm{Zr}^{\star} \\
\mathrm{ppm}\end{array}$ & $\begin{array}{c}\text { Pulp Wt } \\
\text { grams }\end{array}$ & $\begin{array}{c}\text { Lab } \\
\text { Report }\end{array}$ \\
\hline 1181 & 0.89 & 1.28 & 11.7 & 25.1 & 730 & 13.2 & 56.5 & $<0.002$ & 0.03 & 1.59 & 1 & 1.80 & 214.0 & 0.76 & $<0.05$ & 9.0 & 0.465 & 0.36 & 2.3 & 124 & 1.7 & 16.1 & 68 & 72.3 & 9 & FA04030731 \\
\hline 1192 & 0.95 & 1.27 & 9.2 & 24.3 & 740 & 12.5 & 56.1 & $<0.002$ & 0.04 & 1.16 & 1 & 1.50 & 224.0 & 0.60 & $<0.05$ & 8.2 & 0.382 & 0.33 & 2.1 & 113 & 1.1 & 15.6 & 86 & 66.3 & 42 & FA04030731 \\
\hline 1194 & 0.77 & 1.37 & 10.8 & 27.6 & 760 & 13.4 & 59.6 & $<0.002$ & 0.03 & 1.16 & & 1.70 & 236.0 & 0.70 & $<0.05$ & 8.6 & 0.434 & 0.35 & 2.4 & 123 & 1.3 & 17.6 & 76 & 70.5 & 32 & A04030731 \\
\hline & 0.71 & & 0.8 & 26.3 & 710 & 11.6 & 53.1 & $<0,002$ & 0.02 & 1.02 & & 1.50 & 230.0 & 0.71 & $<0.05$ & 8.7 & 0.444 & 0.31 & 2.3 & 116 & 14 & 17.5 & 68 & 74.1 & & A04030731 \\
\hline 1197 & 1.10 & 1.24 & 10.2 & 30.8 & 870 & 14.6 & 59.2 & $<0.002$ & 0.03 & 1.34 & 1 & 1.70 & 220.0 & 0.64 & $<0.05$ & 8.7 & 0.415 & 0.34 & 2.4 & 126 & 1.2 & 18.7 & 77 & 72.5 & 28 & FA04030731 \\
\hline 1198 & 0.98 & 1.20 & 10.0 & 25.7 & 820 & 12.2 & 47.9 & $<0.002$ & 0.02 & 2.02 & 1 & 1.60 & 217.0 & 0.64 & $<0.05$ & 8.7 & 0.425 & 0.28 & 2.6 & 124 & 1.0 & 18.0 & 63 & 72.2 & 74 & FA04030731 \\
\hline 1195 & 1.07 & 0.80 & 9.8 & 30.5 & 980 & 13.0 & 57.4 & $<0.002$ & 0.03 & 1.15 & 1 & 1.70 & 146.5 & 0.63 & $<0.05$ & 7.5 & 0.429 & 0.39 & 2.8 & 162 & 1.2 & 16.0 & 77 & 73.4 & $\sqrt{37}$ & \\
\hline 1201 & 0.99 & 0.91 & 10.2 & 38.6 & 900 & 11.5 & 48.3 & $<0.002$ & 0.04 & 1.19 & & 1.50 & 160.0 & 0.64 & $<0.05$ & 6.4 & 0.463 & 0.35 & 2.4 & 142 & 1.0 & 16.8 & 98 & 71.2 & 3 & FA04030731 \\
\hline & 1.68 & 79 & 3.9 & 44.8 & 1360 & 13.1 & 61.5 & $<0.002$ & 0.04 & 2.19 & & 2.00 & 158.5 & 0.80 & \begin{tabular}{|l|}
0.07 \\
\end{tabular} & 7.0 & 0.592 & 0.47 & 3.9 & 216 & 1. & 22.3 & 114 & 87.8 & & FA04030731 \\
\hline 1203 & 0.69 & 0.90 & 8.9 & 28.2 & 1020 & 10.9 & 46.6 & $<0.002$ & 0.02 & 1.21 & . & 1.40 & 166.5 & 0.59 & $<0.05$ & 6.6 & 0.398 & 0.31 & 2.6 & 132 & 1.0 & 18.0 & 80 & 69.2 & & A04030731 \\
\hline 1204 & 0.86 & 0.98 & 10.1 & 32.1 & 900 & 12.4 & 53.1 & $<0.002$ & 0.03 & 1.23 & & 1.50 & 173.0 & 0.66 & $<0.05$ & 7.5 & 0.445 & 0.34 & 2.5 & 138 & 1.2 & 17.2 & 88 & 71.7 & 3 & FA04030731 \\
\hline & & 1.07 & 10.9 & 27.1 & 800 & 11.2 & 49.0 & $<0.002$ & 0.02 & 1.09 & & 1.60 & 184.5 & 0.75 & $<0.05$ & 8.3 & 0.473 & 0.31 & 2.5 & 128 & 1.1 & 17.2 & & 73.2 & & \\
\hline 120 & 0.68 & 1.12 & 11.2 & 27.6 & 740 & 11.2 & 51.0 & $<0.002$ & 0.02 & 1.00 & 7 & 1.60 & 191.5 & 0.69 & $<0.05$ & 9.0 & 0.476 & 0.31 & 2.5 & 121 & 1.6 & 17.4 & 85 & 76.2 & & FA04030731 \\
\hline 120 & 0.84 & 1.04 & $\begin{array}{ll}10.8 \\
\end{array}$ & 32.7 & 870 & 12.0 & 57.4 & $<0.002$ & 0.03 & 1.16 & & 1.70 & 182.0 & 0.68 & $<0.05$ & 8.3 & 0.449 & 0.32 & 2.5 & 130 & 1.4 & 17.3 & 89 & 75.0 & 3 & FA04030731 \\
\hline & 0.76 & 1.08 & \begin{tabular}{|l|}
10.7 \\
\end{tabular} & 30.9 & 840 & 11.5 & 54.4 & $<0.002$ & 0.03 & 1.18 & & 1.60 & 186.0 & 0.67 & $<0.05$ & 8.4 & 0.452 & 0.32 & 2.4 & 126 & 1.4 & 16.5 & 91 & 69.1 & & A04030731 \\
\hline & 0.91 & 0.91 & 8.8 & 21.9 & 760 & 12.4 & 55.3 & $<0.002$ & 0.05 & 1.12 & & 1.60 & 157.0 & 0.55 & 0.05 & 6.5 & 0.347 & 0.37 & 2.6 & 131 & 1. & 14.6 & 90 & 64.9 & 1 & FA04033 \\
\hline & 1.27 & 0.72 & 6.9 & 22.5 & 710 & 13.6 & 55.0 & 0.002 & 0.03 & 1.20 & & 1.50 & 131.0 & 0.42 & 0.06 & 6.6 & 0.290 & 0.35 & 2.4 & 134 & 0. & 13.8 & & 51.4 & & 7731 \\
\hline & 1.28 & 0.87 & 8.9 & 27.4 & 970 & 14.5 & 66.8 & $<0.002$ & 0.05 & 1.29 & 2 & 1.80 & 160.0 & 0.57 & 0.06 & 7.7 & & 0.41 & 2.7 & 142 & 1. & 17.0 & 81 & 65.8 & & \\
\hline $121 !$ & 5.27 & 0.53 & \begin{tabular}{|l|}
11.4 \\
\end{tabular} & 52.2 & 1340 & 13.8 & 70.7 & 0.007 & 0.08 & 2.36 & & 2.20 & 124.0 & 0.75 & 0.11 & 8.7 & 0.455 & 0.76 & 3.0 & 188 & 1.5 & 22.6 & 163 & 105.0 & 25 & FA04030731 \\
\hline 121 & 4.69 & 0.54 & \begin{tabular}{|l|}
9.9 \\
\end{tabular} & 44.1 & 1220 & 13.9 & 63.8 & 0.005 & 0.04 & 1.98 & & 2.40 & 120.0 & 0.61 & 0.06 & 7.6 & 0.498 & 0.70 & 2.7 & 179 & 1.0 & 19.7 & 120 & 83.8 & & 30731 \\
\hline & 2.13 & .83 & 13.9 & 27.1 & 890 & 13.8 & 66.7 & 0.002 & 0.03 & 1.81 & & 2.00 & 158.5 & 0.85 & $<0.05$ & 8.9 & 0.585 & 0.52 & 2.4 & 15 & 1. & 16.0 & 88 & 81. & & \\
\hline 121 & 2.14 & 0.80 & \begin{tabular}{|l|l}
14.8 \\
\end{tabular} & 27.5 & 860 & 12.8 & 61.8 & 0.002 & 0.02 & 1.63 & & 1.90 & 163.0 & \begin{tabular}{|l|l|}
0.87 \\
\end{tabular} & $<0.05$ & 9.1 & 0.682 & 0.44 & 2.4 & 156 & 1.2 & 17.1 & 8 & 90.5 & & FA04030731 \\
\hline 12 & 1.60 & 0.80 & 12.0 & 25.0 & 720 & 11.6 & 57.3 & 0.002 & 0.02 & 1.64 & & 1.80 & 139.5 & 0.70 & $<0.05$ & 7.6 & 0.509 & 0.36 & 2.0 & 138 & 1.0 & 13.7 & 1 & 72.8 & & 30731 \\
\hline & 1.54 & 0.89 & 12.5 & 25.9 & 780 & 13.4 & 62.0 & $<0.002$ & 0.02 & 1.86 & 2 & 1.70 & 153.0 & \begin{tabular}{|l|l|}
0.74 \\
\end{tabular} & $<0.05$ & 9.3 & 0.478 & 0.42 & 2.1 & 139 & 1.1 & 14.8 & 8 & 69.0 & & 0731 \\
\hline 1222 & 1.00 & 1.07 & 13.3 & 25.4 & 700 & 12.3 & 55.3 & 0.002 & 0.02 & 1.32 & & 1.70 & 187.5 & \begin{tabular}{|l|}
0.86 \\
\end{tabular} & $<0.05$ & 12.4 & 0.597 & 0.35 & 2.6 & 126 & 1. & 18.2 & 7 & 84.8 & & FA04030731 \\
\hline & 1.29 & \begin{tabular}{|l|}
0.93 \\
\end{tabular} & 12.0 & 24.5 & 710 & 11.6 & 56.2 & 0.002 & 0.02 & 1.41 & I & 1.60 & 162.5 & \begin{tabular}{|l|l|}
0.74 \\
\end{tabular} & $<0.05$ & 9.7 & 0.532 & 0.35 & 2.1 & 130 & 1. & 15.6 & 7 & 72 . & & 731 \\
\hline 122 & 1.15 & 1.05 & 12.3 & 26.1 & 700 & 12.4 & 57.6 & $<0.002$ & 0.02 & 1.35 & & 1.80 & 177.0 & \begin{tabular}{|l|l|}
0.78 \\
\end{tabular} & $<0.05$ & 9.5 & 0.531 & 0.37 & 2.3 & 132 & 1. & 16.5 & 7 & 80.8 & & 30731 \\
\hline 1226 & 1.11 & 1.12 & 12.8 & 25.5 & 730 & 11.4 & 55.7 & $<0.002$ & 0.01 & 1.34 & & 1.70 & 190.5 & \begin{tabular}{|l|l|}
0.84 \\
\end{tabular} & $<0.05$ & 10.9 & 0.557 & 0.32 & 2.3 & 128 & 1. & 17.0 & 7 & 76.5 & & FA04030731 \\
\hline & 1.47 & 1.04 & $\overline{0.8}$ & 29.4 & 800 & 13.8 & 65.1 & 0.002 & $\overline{0.1}$ & 1.46 & & 1.70 & 176.5 & \begin{tabular}{|l|l|}
0.67 \\
\end{tabular} & $<0.05$ & 9.9 & 0.448 & 0.40 & 2.4 & 141 & & 17.2 & 82 & 72 & & \\
\hline 1229 & 3.84 & 0.55 & 10.6 & 45.0 & 840 & 19.1 & 89.2 & 0.011 & 0.16 & 2.88 & & 2.20 & 171.0 & \begin{tabular}{|l|l|}
0.68 \\
\end{tabular} & \begin{tabular}{|l|}
0.07 \\
\end{tabular} & 10.5 & 0.363 & 0.94 & 4.0 & 152 & 1. & \begin{tabular}{|l|}
18.0 \\
\end{tabular} & 128 & 93. & & FA04030731 \\
\hline 1230 & 1.05 & 1.06 & 12.7 & 29.8 & 570 & 22.4 & 107.5 & $<0.002$ & 0.03 & 1.41 & 2 & 2.90 & 195.5 & \begin{tabular}{|l|l|}
0.84 \\
\end{tabular} & 0.05 & 13.7 & 0.471 & 0.55 & 2.4 & 121 & 1. & 14.9 & 103 & 81.1 & & FA04030731 \\
\hline & 1.67 & 0.81 & 13.2 & 51.2 & 690 & 21.1 & 114.0 & 0.003 & 0.05 & 2.45 & & 2.50 & 208.0 & 1.04 & 0.05 & 15.3 & 0.453 & 0.65 & 2.9 & 121 & 1. & 17.4 & 102 & 96.6 & & \\
\hline 1233 & 1.49 & 0.81 & 12.2 & 37.1 & 680 & 15.4 & 98.9 & 0.003 & 0.05 & 1.84 & & 2.20 & 159.0 & 0.99 & $<0.05$ & 13.4 & 0.459 & 0.61 & 2.9 & 120 & 1.2 & \begin{tabular}{|l|}
17.4 \\
\end{tabular} & 122 & 94.1 & 2 & FA04030731 \\
\hline 1234 & 2.70 & 0.86 & 10.3 & 34.9 & 680 & 16.6 & 76.7 & 0.011 & 0.09 & 1.82 & & 2.00 & 207.0 & 0.83 & 0.05 & 9.5 & 0.410 & 0.65 & 3.2 & 144 & 1. & 19.6 & 98 & 78.9 & 2 & FA04030731 \\
\hline & 4.19 & 0.79 & \begin{tabular}{|l|}
9.2 \\
\end{tabular} & 44.1 & 9 & & 67.2 & & & 2.23 & & 1.80 & 173.5 & \begin{tabular}{|l|}
0.75 \\
\end{tabular} & 0.06 & 8.7 & & 0.74 & 2.9 & 149 & & \begin{tabular}{|l|}
17.7 \\
\end{tabular} & 215 & 74. & & \\
\hline 1236 & 5.54 & 0.85 & 10.2 & 42.5 & 930 & 15.8 & 70.2 & 0.005 & 0.10 & 3.01 & 4 & 1.80 & 184.0 & \begin{tabular}{|l|l|}
0.82 \\
\end{tabular} & 0.06 & 9.1 & 0.408 & 0.81 & 3.1 & 166 & 1. & 17.6 & 151 & 73.9 & 21 & FA04030731 \\
\hline 1238 & 4.99 & 0.72 & 11.5 & 44.0 & 940 & 15.2 & 81.5 & 0.005 & 0.08 & 2.70 & & 2.30 & 156.0 & \begin{tabular}{|l|}
0.91 \\
\end{tabular} & 0.06 & 10.5 & 0.431 & 0.74 & 3.1 & 164 & 1.2 & \begin{tabular}{|l|}
18.4 \\
\end{tabular} & 168 & 87.6 & 2. & A04030731 \\
\hline & 3.80 & 0.76 & & 42.7 & 870 & & 78.0 & & 0.06 & 2.16 & & 2.10 & 157.5 & \begin{tabular}{|l|}
0.94 \\
\end{tabular} & 0.06 & & 0.441 & 0.68 & & & & 17.3 & 123 & 79.8 & & \\
\hline 1241 & 3.11 & 0.96 & 12.0 & 39.3 & 830 & 15.0 & 75.3 & 0.003 & 0.07 & 2.02 & 3 & 2.00 & 186.0 & 0.94 & 0.05 & 11.3 & 0.457 & 0.65 & 3.0 & 137 & 1. & \begin{tabular}{|l|l|}
19.4 \\
\end{tabular} & 126 & 86.6 & 25 & FA04030731 \\
\hline 1242 & 2.79 & 0.95 & 10.6 & 35.8 & 780 & 13.1 & 68.6 & 0.003 & 0.06 & 1.80 & & 1.80 & 176.5 & \begin{tabular}{|l|}
0.86 \\
\end{tabular} & $<0.05$ & 10.0 & 0.420 & 0.59 & 2.7 & 129 & & \begin{tabular}{|l|}
16.9 \\
\end{tabular} & 126 & 71.4 & & A04030731 \\
\hline & 2.69 & 0.99 & 10.6 & 39.0 & 790 & 14.4 & 73.7 & 0.004 & & 1.91 & 3 & 2.00 & 188.0 & \begin{tabular}{|l|}
0.81 \\
\end{tabular} & & 10.3 & & 0.65 & 2.8 & 13 & 1 & & 168 & 73.4 & 2 & 030731 \\
\hline 1245 & 1.03 & 1.11 & 11.1 & 26.6 & 620 & 14.2 & 66.5 & $<0.002$ & 0.03 & 0.85 & 1 & 2.10 & 161.5 & \begin{tabular}{|l|}
0.94 \\
\end{tabular} & $<0.05$ & 9.5 & 0.464 & 0.45 & 2.2 & 128 & 1.4 & 14.6 & 77 & 68.8 & 21 & FA04030731 \\
\hline 1246 & 1.00 & 1.15 & 11.0 & 30.2 & 600 & 13.8 & 67.0 & 0.002 & 0.03 & 0.81 & 1 & 7.50 & 166.5 & \begin{tabular}{|l|l|}
0.87 \\
\end{tabular} & $<0.05$ & \begin{tabular}{|l|}
8.4 \\
\end{tabular} & 0.485 & 0.44 & 2.1 & 134 & 1.2 & $\mid 16.0$ & 111 & 69.3 & 21 & FA04030731 \\
\hline
\end{tabular}


Table 1. Location and New Trace Element Geochemical Results for 902 Stream-Sediment Samples collected in the Livengood Area, Livengood Quadrangle, Alaska IS = Insufficient Sample for Analysis

\begin{tabular}{|c|c|c|c|c|c|c|c|c|c|c|c|c|c|c|c|c|c|c|c|c|c|c|c|c|c|c|c|}
\hline MPLE & TM E & TM N & $\begin{array}{c}\mathrm{Au} \\
\mathrm{ppm}\end{array}$ & $\begin{array}{l}\mathrm{Ag} \\
\mathrm{ppm}\end{array}$ & $\begin{array}{l}\text { Al } \\
\%\end{array}$ & $\begin{array}{c}\text { As } \\
\text { ppm }\end{array}$ & $\begin{array}{l}\mathrm{Ba}^{*} \\
\mathrm{ppm}\end{array}$ & $\begin{array}{c}\mathrm{Be} \\
\mathrm{ppm} \\
\end{array}$ & $\begin{array}{c}\mathrm{Bi} \\
\mathrm{ppm}\end{array}$ & $\begin{array}{c}\mathrm{Ca} \\
\% \\
\end{array}$ & $\begin{array}{c}\mathrm{Cd} \\
\mathrm{ppm}\end{array}$ & $\begin{array}{r}\text { Ce } \\
\text { ppm }\end{array}$ & $\begin{array}{l}\text { Co } \\
\text { ppm }\end{array}$ & $\begin{array}{l}\mathrm{Cr}^{\star} \\
\mathrm{ppm}\end{array}$ & $\begin{array}{r}\text { Cs } \\
\text { ppm }\end{array}$ & $\begin{array}{r}\mathrm{Cu} \\
\mathrm{ppm}\end{array}$ & $\begin{array}{c}\mathrm{Fe} \\
\% \\
\end{array}$ & $\begin{array}{c}\mathrm{Ga} \\
\mathrm{ppm}\end{array}$ & $\begin{array}{l}\mathrm{Ge} \\
\mathrm{ppm}\end{array}$ & $\begin{array}{c}\mathrm{Hf} \\
\mathrm{ppm} \\
\end{array}$ & $\begin{array}{l}\mathrm{Hg} \\
\mathrm{ppm}\end{array}$ & $\begin{array}{c}\text { In } \\
\text { ppm }\end{array}$ & $\begin{array}{l}\mathrm{K} \\
\% \\
\end{array}$ & $\begin{array}{r}\mathrm{La} \\
\mathrm{ppm}\end{array}$ & $\begin{array}{c}\mathrm{Li} \\
\mathrm{ppm} \\
\end{array}$ & $\begin{array}{c}\mathrm{Mg} \\
\% \\
\end{array}$ & $\begin{array}{l}\mathrm{Mn} \\
\mathrm{ppm}\end{array}$ \\
\hline 247 & 3837 & 51418 & $<0.005$ & \begin{tabular}{|l|l|}
0.12 \\
\end{tabular} & & 6.9 & 710 & $\begin{array}{l}1.13 \\
\end{array}$ & 0.15 & & \begin{tabular}{|l}
0.23 \\
\end{tabular} & & 132 & 87 & 3.16 & 19.4 & & & 0.14 & \begin{tabular}{|l|}
1.5 \\
\end{tabular} & 0.25 & 0.049 & \begin{tabular}{|l|l}
1.22 \\
\end{tabular} & 30.3 & 28.7 & 0.93 & 519 \\
\hline$\overline{48}$ & & & 05 & & 5.93 & 6. & 50 & & & & \begin{tabular}{|l|}
0.18 \\
\end{tabular} & 65. & 11. & & 14 & 4. & 3.09 & 13.35 & 0.15 & 1.5 & 0.25 & 0.042 & \begin{tabular}{|l|l|}
1.07 \\
\end{tabular} & 33.0 & 4.0 & 0.91 & 491 \\
\hline & & & 006 & 13 & 6.37 & 9.2 & 760 & 27 & 0.14 & 18 & & 5.2 & 11.5 & 8 & 2.91 & 16.9 & 3.31 & 14.90 & 0.15 & $\overline{14}$ & 0.21 & .046 & 1.26 & 32.0 & 5.2 & 0.89 & $\overline{04}$ \\
\hline & & & & & 6.86 & 10.4 & 20 & & & & & & & & & & & & & & & & & & & 0098 & 07 \\
\hline & & & .005 & 17 & 6.61 & 8.4 & 750 & 25 & 0.17 & 1.10 & \begin{tabular}{|l|}
0.38 \\
\end{tabular} & 8.3 & 13. & 9 & 4.64 & 31.2 & 3.30 & 15.60 & 0.16 & 1.6 & 0.29 & 0.050 & \begin{tabular}{|l|}
1.27 \\
\end{tabular} & 34.4 & 34.0 & 0.96 & 791 \\
\hline & & & 009 & 0.12 & \begin{tabular}{|l|l}
6.50 \\
\end{tabular} & 7.6 & 770 & 1.40 & 0.15 & 1.42 & \begin{tabular}{|l|}
0.23 \\
\end{tabular} & 63.9 & 11. & & 3.19 & 22.2 & 3.02 & 15.25 & 0.15 & 1.4 & 0.21 & 0.045 & \begin{tabular}{|l}
1.32 \\
\end{tabular} & 32.7 & 26.7 & 0.96 & 311 \\
\hline & & & & & \begin{tabular}{|l|l|}
6.66 \\
\end{tabular} & 7.7 & 690 & & & & & & & & & & 3.12 & 16.40 & 016 & & & & & 451 & & 077 & \\
\hline & & & .005 & 0.08 & 7.73 & 10.2 & 870 & 1.99 & 0.21 & 0.73 & 0.35 & 6.1 & $\begin{array}{l}17.7 \\
\end{array}$ & 8 & 3.3 & 24. & 3.94 & 19.80 & 0.19 & 1.9 & 0.19 & .057 & \begin{tabular}{|l|}
2.11 \\
\end{tabular} & 43.6 & 51.2 & 0.87 & 346 \\
\hline & & & . & 08 & 7.20 & 6.9 & 780 & .72 & 0.21 & 01 & 0.22 & 87.8 & 16. & & 3.44 & 3.2 & 3.36 & 18.05 & 0.18 & 2.0 & 0.15 & 0.051 & \begin{tabular}{|l|}
1.81 \\
\end{tabular} & 43.7 & 42.4 & 0.85 & 06 \\
\hline & & & .008 & .08 & \begin{tabular}{|l|l}
7.22 \\
\end{tabular} & 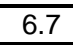 & 750 & 69 & 0.19 & & \begin{tabular}{|l|l|}
0.19 \\
\end{tabular} & 99.2 & 15. & & 3.08 & 20.0 & 3.42 & 17.85 & 0.20 & 2.7 & 0.33 & 0.048 & \begin{tabular}{|l|}
1.91 \\
\end{tabular} & 49.4 & 5.7 & 0.82 & 11 \\
\hline & & & $<0.005$ & 0.07 & \begin{tabular}{|l}
6.37 \\
\end{tabular} & 6. & 670 & 1.30 & 0.15 & 1.26 & \begin{tabular}{|l|l|}
0.17 \\
\end{tabular} & 79.8 & 11. & & 2.5 & 18. & 2.89 & 15.20 & 0.17 & 1.7 & 0.14 & 0.046 & \begin{tabular}{|l}
1.46 \\
\end{tabular} & 40.5 & 28.6 & 0.88 & 330 \\
\hline & & & .005 & 08 & \begin{tabular}{|l|l}
6.69 \\
\end{tabular} & 1. & 750 & $\bar{L}$ & & 1.12 & \begin{tabular}{|l|l|}
0.22 \\
\end{tabular} & 85.9 & 14. & & 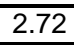 & . & 3.21 & 16.05 & 0.18 & 2.1 & 0.18 & 0.045 & \begin{tabular}{|l|}
1.62 \\
\end{tabular} & 42.8 & 37.0 & \begin{tabular}{|l}
0.84 \\
\end{tabular} & 777 \\
\hline & & & .005 & 09 & 60 & 7.3 & 740 & & & 40 & 22 & 7 & 12 & & .5 & $\overline{0}$ & 305 & 15.30 & 0.16 & & & .046 & 1.46 & & 9.4 & 0.89 & 17 \\
\hline & & & .007 & 09 & \begin{tabular}{|l|l|}
6.40 \\
\end{tabular} & 6.5 & 730 & 1.32 & 0.16 & 1.40 & \begin{tabular}{|l|}
0.29 \\
\end{tabular} & 66.4 & 12. & 7 & 2.57 & 20.1 & 2.95 & 15.40 & 0.16 & 1.6 & 0.21 & .046 & 1.50 & 32.9 & 29.3 & 0.89 & 708 \\
\hline & & & .005 & & \begin{tabular}{|l|l|}
6.16 \\
\end{tabular} & $20 . \varepsilon$ & 820 & & & 0.42 & & & & & & & 3.24 & 16.60 & 0.11 & & & & \begin{tabular}{|l|}
1.69 \\
\end{tabular} & & 5.7 & 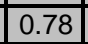 & 437 \\
\hline & & & 0.005 & 10 & \begin{tabular}{|l|l|}
6.73 \\
\end{tabular} & 8.0 & 780 & $\overline{39}$ & 0.20 & 0.94 & 33 & 78.7 & 15.5 & 8 & 3.64 & 18.8 & 3.16 & 16.30 & 0.18 & & 0.25 & .048 & 1.52 & 38.9 & 5.5 & 0.80 & 1110 \\
\hline & & & .005 & 06 & 6.57 & 6.7 & 730 & .33 & 16 & 86 & 21 & 90.5 & 14. & & 3.41 & 4. & 2.88 & 6.20 & 0.17 & 2. & 0.14 & 045 & 1.58 & 42.5 & 33.6 & 0.78 & 722 \\
\hline & & & 014 & $\overline{09}$ & 77 & & 7 & & & 89 & & & & & & & & .55 & & & & 049 & 1.60 & & 1.5 & 7 & $\overline{86}$ \\
\hline & & & .011 & 0.08 & .71 & 6.5 & 750 & 1.55 & 0.17 & 1.10 & \begin{tabular}{|l|}
0.25 \\
\end{tabular} & 112. & 15. & & 3.18 & 19. & 3.28 & 17.15 & 0.19 & 2.4 & 0.17 & $\overline{049}$ & \begin{tabular}{|l|}
1.61 \\
\end{tabular} & 52.9 & 34.5 & 0.89 & 775 \\
\hline & & & .005 & & 75 & & 780 & & & 04 & & 0. & & & & & 3.24 & 16.45 & 0.2 & 2. & 0.1 & 041 & 1.62 & 52 & 31.6 & \begin{tabular}{|l}
0.87 \\
\end{tabular} & 368 \\
\hline & & & .005 & 09 & & 7.2 & 770 & 1.53 & & 97 & & & & & 3.2 & & & 6.75 & 0.17 & & & 049 & 1.68 & .2 & \begin{tabular}{|l|l|}
32.1 \\
\end{tabular} & 0.86 & 03 \\
\hline & & & 0.005 & 0.07 & 6.54 & 7.3 & 750 & 1.46 & 0.15 & 0.75 & \begin{tabular}{|l|l|}
0.14 \\
\end{tabular} & 98.6 & 14. & 6 & 2.86 & 16. & 3.35 & 16.10 & 0.18 & 2.5 & 0.15 & .042 & \begin{tabular}{|l|l}
1.72 \\
\end{tabular} & 47.4 & \begin{tabular}{|l|l|}
31.7 \\
\end{tabular} & 0.81 & 680 \\
\hline & & & 016 & 09 & 61 & 7.7 & 770 & & & 1.60 & & & & & 2.2 & 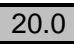 & 3.1 & 5.10 & 0.1 & 1.2 & 0.36 & .044 & 1.40 & 32.5 & 23.4 & 1.01 & 570 \\
\hline & & & .005 & & & & 810 & & & 37 & & & & & & & 3.20 & & & & & & 1. & 44 & \begin{tabular}{|l|}
33.1 \\
\end{tabular} & 0.95 & 58 \\
\hline & & & 010 & 27 & .51 & & 880 & & 0.2 & 0.78 & & & 24 & & 4.2 & 49 & 3.7 & 8.00 & 0.0 & 2. & 0.22 & .060 & 1.32 & 34. & 28.0 & 0.83 & 525 \\
\hline & & & & 47 & & & 9 & & & 83 & & & & & & & 3.7 & .25 & 0.4 & & .3 & 061 & 1.37 & 34.9 & 6.4 & 0.84 & 1415 \\
\hline & & & $\overline{009}$ & 30 & 5.44 & 12.9 & 880 & & 0.22 & 72 & \begin{tabular}{|l|}
0.88 \\
\end{tabular} & & & & 38 & 62. & 3.27 & 4.25 & 0.08 & 2. & 0.34 & 50 & 1.11 & 30.7 & 9.8 & 0.67 & 724 \\
\hline & & & & & 34 & $\overline{3.6}$ & 190 & & & 93 & & & 16. & & 4.0 & 2 & 3.75 & 4.15 & & 2. & 0.22 & .050 & \begin{tabular}{|l|}
1.14 \\
\end{tabular} & 33.1 & 20.1 & 0.72 & 1080 \\
\hline & & & & & 5.40 & & 240 & & & & & & & & & & & & & & & & & & $E \perp$ & 0.1 & 725 \\
\hline & & & & & & & & & & & & & & & & & & & & & & & & & & $\overline{0.8}$ & 791 \\
\hline & & & .012 & 0.28 & \begin{tabular}{|l|}
5.89 \\
\end{tabular} & 1.4 & 840 & & & 0.85 & 43 & 6 & 16. & & 3.3 & $\pi$ & 3.32 & 5.10 & 0.08 & 2.4 & 0.24 & .051 & 1.24 & 32.2 & 3.2 & 0.79 & 556 \\
\hline & & & & & 5.39 & & & & & 0.79 & & & & & & & & & & 2.3 & & & .14 & & 22.4 & 0.10 & 40 \\
\hline & & & & & 5.53 & & 040 & & & & & & & & & & 3.22 & & & & $\overline{0.2}$ & & & 33 & & 0.74 & 1265 \\
\hline & & & 0.015 & 0.24 & 5.89 & 10.8 & 1000 & & 0. & 1.30 & 0.90 & & 19. & 100 & 3.3 & 2. & 3.57 & 14.35 & 0.10 & 2.1 & 0.17 & 0.053 & 1.12 & 35.2 & 25.8 & 0.94 & 1270 \\
\hline & & & & & & 10 & 650 & & & & & & & & & & & & & & & & 1.10 & 28.1 & o. & 0.12 & 436 \\
\hline & & & .005 & & 5.48 & 7.2 & 650 & & & & & & & & 5. & & 2.53 & 1.50 & & 1. & & $.04 !$ & 1.16 & 33.2 & 7 & 0.86 & 525 \\
\hline & & 7 & $<0.005$ & 0.19 & 6.07 & 8. & 730 & & 0. & 1.24 & \begin{tabular}{|l|}
0.21 \\
\end{tabular} & 81.5 & 13. & 112 & 3.9 & & 3.16 & 4.65 & 0.1 & 2.1 & 0.18 & 051 & 1.34 & 40.2 & 30.4 & 1.08 & 741 \\
\hline & & & & & & & & & & & & & & & & & & & & & & & & & 5 & 80 & 60 \\
\hline 1328 & & & 0.024 & 0.15 & 5.51 & 9.8 & 700 & & 0. & 1.43 & \begin{tabular}{|l|}
0.36 \\
\end{tabular} & 108.5 & & 110 & 2. & & 3.15 & 13.90 & 14 & 2.1 & 0.38 & .049 & 1.13 & 52.1 & 3.4 & 0.92 & 500 \\
\hline & & & $<0.005$ & \begin{tabular}{|l|}
0.14 \\
\end{tabular} & 5.90 & 9.3 & 780 & 1. & 0. & 1.40 & \begin{tabular}{|l|}
0.39 \\
\end{tabular} & 68 & 23 & 8 & 2. & & 3.03 & 14.20 & 0 & 2.0 & 0.28 & .045 & $\begin{array}{ll}1.22 \\
\end{array}$ & 33.2 & 3.4 & 0.94 & 1655 \\
\hline & & & & & & 8 & 780 & & & & & & & & & & & & & 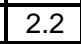 & & & 1.24 & 38.1 & & 1.00 & 745 \\
\hline 133 & & 7239624 & $<0.005$ & 0.19 & 6.10 & 6.1 & 740 & 1.50 & 0.17 & 1.62 & 0.20 & 87.7 & 10. & 71 & 4.8 & 13.8 & 2.8 & 14.90 & & 1.7 & 0.24 & .047 & 1.28 & 43.5 & 25.7 & 1.05 & 404 \\
\hline & & & & & & 14.4 & 790 & & & & & 14.0 & 14.4 & & & & 4.34 & & & 2.1 & 0.24 & & 1.10 & 66.2 & & 1.34 & \\
\hline
\end{tabular}


Table 1. Location and New Trace Element Geochemical Results for 902 Stream-Sediment Samples collected in the Livengood Area, Livengood Quadrangle, Alaska IS = Insufficient Sample for Analysis

\begin{tabular}{|c|c|c|c|c|c|c|c|c|c|c|c|c|c|c|c|c|c|c|c|c|c|c|c|c|c|c|}
\hline SAMPLE & $\begin{array}{l}\text { Mo } \\
\mathrm{ppm}\end{array}$ & $\begin{array}{c}\mathrm{Na} \\
\% \\
\end{array}$ & $\begin{array}{l}\mathrm{Nb} \\
\mathrm{ppm}\end{array}$ & $\begin{array}{c}\mathrm{Ni} \\
\mathrm{ppm}\end{array}$ & $\begin{array}{c}\mathrm{P} \\
\mathrm{ppm}\end{array}$ & $\begin{array}{l}\mathrm{Pb} \\
\mathrm{ppm}\end{array}$ & $\begin{array}{l}\mathrm{Rb} \\
\mathrm{ppm}\end{array}$ & $\begin{array}{l}\mathrm{Re} \\
\mathrm{ppm}\end{array}$ & $\begin{array}{l}\mathrm{S} \\
\%\end{array}$ & $\begin{array}{l}\mathrm{Sb} \\
\mathrm{ppm}\end{array}$ & $\begin{array}{l}\text { Se } \\
\mathrm{ppm}\end{array}$ & $\begin{array}{l}S^{\text {Sn }} \\
\mathrm{ppm}\end{array}$ & $\begin{array}{c}\mathrm{Sr} \\
\mathrm{ppm}\end{array}$ & $\begin{array}{l}T^{*} \\
\mathrm{ppm}\end{array}$ & $\begin{array}{c}\text { Te } \\
\text { ppm }\end{array}$ & $\begin{array}{c}\text { Th } \\
\text { ppm }\end{array}$ & $\begin{array}{l}\mathrm{Ti}^{*} \\
\%\end{array}$ & $\begin{array}{c}\mathrm{Tl} \\
\mathrm{ppm}\end{array}$ & $\begin{array}{c}U \\
\mathrm{ppm}\end{array}$ & \begin{tabular}{|c|}
$\mathrm{V}$ \\
$\mathrm{ppm}$
\end{tabular} & $\begin{array}{l}W^{*} \\
\mathrm{ppm}\end{array}$ & $\begin{array}{c}\mathrm{Y} \\
\mathrm{ppm}\end{array}$ & $\begin{array}{c}\mathrm{Zn} \\
\mathrm{ppm}\end{array}$ & $\begin{array}{l}\mathrm{Zr}^{*} \\
\mathrm{ppm}\end{array}$ & $\begin{array}{c}\text { Pulp Wt } \\
\text { grams }\end{array}$ & $\begin{array}{c}\text { Lab } \\
\text { Report }\end{array}$ \\
\hline 1247 & 0.84 & 1.23 & 10.3 & 30.0 & 570 & 14.0 & 60.5 & $<0.002$ & 0.04 & 0.88 & 1 & 1.90 & 183.5 & 0.81 & $<0.05$ & 7.6 & 0.448 & 0.42 & 1.9 & 127 & 1.0 & 16.3 & 83 & 61.2 & 33 & FA04030731 \\
\hline 1248 & 0.62 & 26 & 10.2 & 26.1 & 530 & 11.2 & 51.9 & $<0.002$ & 0.02 & 0.74 & 1 & 1.60 & 31.5 & 0.84 & $<0.05$ & 8.8 & 0.474 & 0.33 & 2.0 & 116 & 1.0 & 15.7 & 78 & 62.7 & 23 & FA04030731 \\
\hline 1249 & 0.65 & 22 & 9.9 & 28.7 & 690 & 13.7 & 63.0 & $<0.002$ & 0.05 & 0.85 & 1 & 1.70 & 190.0 & 0.80 & $<0.05$ & 8.2 & 0.426 & 0.39 & 2.0 & 121 & 1.0 & 16.6 & 73 & 60.8 & $\overline{42}$ & FA04030731 \\
\hline 250 & 1.16 & 04 & 10.2 & 36.7 & 780 & 15.0 & 71.1 & $<0.002$ & 0.04 & 1.00 & & 2.00 & 160.0 & 0.83 & $<0.05$ & 8.5 & 0.450 & 0.49 & 2.2 & 143 & 1.2 & 18.4 & 88 & 65.4 & & FA0403 \\
\hline 251 & 1.00 & 13 & 10.4 & 39.5 & 700 & 13.8 & 65.2 & $<0.002$ & 0.03 & 0.93 & 2 & 1.70 & 171.0 & \begin{tabular}{l|l|}
0.79 \\
\end{tabular} & $<0.05$ & 8.6 & 0.450 & 0.41 & 2.1 & 138 & 1.1 & 19.4 & 95 & 68.2 & & \\
\hline 1252 & 0.76 & 1.32 & 10.4 & 31.5 & 610 & 13.0 & 64.0 & $<0.002$ & $\overline{0.02}$ & 0.99 & 1 & 1.80 & 215.0 & \begin{tabular}{|l|}
0.82 \\
\end{tabular} & $<0.05$ & 8.5 & 0.434 & 0.37 & 1.9 & 121 & 1.1 & 17.0 & 74 & 61.9 & & FA04030731 \\
\hline 253 & 0.66 & 95 & 9.8 & 27.5 & 520 & 17.2 & 83.4 & $<0.002$ & 0.03 & 0.88 & 1 & 2.80 & 145.0 & 0.82 & $<0.05$ & 13.0 & 0.403 & 0.49 & 3.0 & 96 & 1.3 & 15.7 & 81 & 85.4 & & 0731 \\
\hline 254 & 0.74 & 96 & 14.6 & 41.5 & 580 & 19.7 & 104.5 & $<0.002$ & 0.03 & 0.73 & 1 & 3.00 & 142.5 & 1.15 & $<0.05$ & 13.5 & 0.426 & 0.53 & 2.8 & 106 & 1.1 & 17.0 & 118 & 83.6 & 21 & 0731 \\
\hline 56 & 0.57 & 0.95 & 13.0 & 35.7 & 560 & 18.2 & 93.3 & $<0.002$ & 0.05 & 0.68 & 1 & 2.30 & \begin{tabular}{|c|}
168.5 \\
\end{tabular} & \begin{tabular}{|l|}
1.05 \\
\end{tabular} & $<0.05$ & 13.1 & 0.438 & 0.52 & 4.0 & 104 & 1.1 & 18.0 & 87 & 84.3 & 2 & FA040 \\
\hline 57 & 52 & 93 & 8 & 34.9 & 480 & 15.6 & 94.9 & $<0.002$ & 0.02 & 0.61 & & 3.10 & 147.0 & \begin{tabular}{|l|}
1.08 \\
\end{tabular} & $<0.05$ & 16.3 & 0.443 & 0.49 & 3.9 & 95 & & 18.9 & & 112.0 & & 7731 \\
\hline 58 & 0.47 & 1.12 & 11.4 & 29.4 & 520 & 13.9 & 71.4 & $<0.002$ & 0.03 & 0.79 & 1 & 1.80 & 190.5 & $\begin{array}{l}0.91 \\
\end{array}$ & $<0.05$ & 111.5 & 0.432 & 0.40 & 2.9 & 97 & 1.2 & 17.1 & 72 & 75.8 & & 731 \\
\hline 59 & 0.56 & 0.99 & 14.6 & 32.1 & 530 & 15.1 & 83.4 & $<0.002$ & 0.05 & 0.71 & 1 & 2.00 & 176.0 & \begin{tabular}{|l|}
1.32 \\
\end{tabular} & $<0.05$ & 13.8 & 0.421 & 0.46 & 3.5 & 97 & 1.1 & 18.2 & 78 & 87.0 & 2 & FA040 \\
\hline & 55 & 15 & 8.9 & 29.7 & 530 & 14.4 & 71.9 & $<0.002$ & 0.04 & 0.81 & & 1.80 & 205.0 & \begin{tabular}{|l|}
0.74 \\
\end{tabular} & $<0.05$ & 10.8 & 0.365 & 0.40 & 2.8 & 101 & & 16.8 & & 67.3 & & \\
\hline 61 & 0.54 & 1.13 & 10.8 & 30.0 & 590 & 14.8 & 75.6 & $<0.002$ & 0.06 & 0.83 & 1 & 1.80 & 213.0 & \begin{tabular}{|l|l|}
0.87 \\
\end{tabular} & $<0.05$ & $\begin{array}{l}9.8 \\
\end{array}$ & 0.403 & 0.40 & 2.7 & 97 & 1.0 & 16.2 & 90 & 69.9 & & FA04 \\
\hline 63 & 0.94 & 0.91 & 14.6 & 43.7 & 620 & 19.8 & 79.8 & 0.004 & 1.15 & 0.73 & 2 & 2.10 & 103.0 & \begin{tabular}{|l|}
1.18 \\
\end{tabular} & $<0.05$ & \begin{tabular}{|l|l|}
10.7 \\
\end{tabular} & 0.415 & 0.44 & 4.0 & 105 & 1.0 & 18.4 & 80 & 86.1 & & 7731 \\
\hline 64 & 8 & 0.96 & 9.2 & 30.0 & 510 & 19.5 & 86.3 & $<0.002$ & 0.04 & 0.80 & & 2.30 & 161.0 & \begin{tabular}{|l|}
0.78 \\
\end{tabular} & $<0.05$ & 11.2 & 0.377 & 0.52 & 5.2 & 102 & & 16.9 & & 79.1 & & \\
\hline 65 & 0.56 & 1.02 & 10.0 & 26.6 & 420 & 17.2 & 82.6 & $<0.002$ & 0.03 & 0.76 & 1 & 1.80 & 152.0 & \begin{tabular}{|c|}
0.84 \\
\end{tabular} & $<0.05$ & 13.5 & 0.399 & 0.48 & 3.6 & 92 & 1.2 & 16.3 & 76 & 90.3 & 3 & $\mathrm{FAOL}$ \\
\hline 56 & 0.61 & 1.00 & 10.6 & 31.5 & 490 & 18.0 & 85.1 & $<0.002$ & 0.03 & 0.81 & & 1.90 & 168.0 & \begin{tabular}{|c|}
0.86 \\
\end{tabular} & $<0.05$ & 12.5 & 0.399 & 0.47 & 3.9 & 96 & & 17.2 & & 86.4 & & \\
\hline & 0.56 & 1.06 & 14.1 & 32.8 & 580 & 16.6 & 80.1 & 00.002 & 0.03 & 0.81 & 1 & 2.10 & 186.0 & \begin{tabular}{|l|}
1.09 \\
\end{tabular} & $<0.05$ & 16.3 & 0.494 & 0.44 & 3.9 & 100 & & 20.6 & & 106.5 & & \\
\hline 69 & 0.48 & 1.04 & \begin{tabular}{|l|l|}
13.8 \\
\end{tabular} & 30.5 & 530 & 15.8 & 80.7 & $<0.002$ & 0.03 & 0.72 & $\sqrt{1}$ & 2.00 & 169.5 & \begin{tabular}{|l|l|}
1.12 \\
\end{tabular} & $<0.05$ & 15.5 & 0.479 & 0.43 & 3.3 & 97 & 1.3 & 18.9 & 84 & 98.8 & 2 & FA04 \\
\hline 70 & 53 & 1.00 & 12.8 & 30.7 & 550 & 16.4 & 83.4 & $<0.002$ & 0.03 & 0.71 & & 2.00 & 163.0 & \begin{tabular}{|l|}
1.02 \\
\end{tabular} & $<0.05$ & 14.5 & .432 & 0.45 & 3.4 & 9 & 1.1 & 18.2 & & 91.9 & & FA04 \\
\hline & 52 & 0.96 & 3.0 & 29.1 & 490 & 13.2 & 81.0 & $<0.002$ & 0.02 & 0.78 & 1 & 1.90 & 130.0 & 1.06 & $<0.05$ & \begin{tabular}{l|l}
14.5 \\
\end{tabular} & 0.428 & 0.4 & 2.9 & 8 & 1. & 16.7 & 77 & 98. & & $\mathrm{FAO}$ \\
\hline 72 & 0.65 & 1.39 & 10.4 & 29.1 & 600 & 13.5 & 62.4 & $<0.002$ & 0.04 & 1.06 & & 1.80 & 235.0 & 0.93 & $<0.05$ & 9.0 & 0.428 & 0.35 & 2.1 & 10 & 1. & 15.8 & & 61.0 & & FA04 \\
\hline & 88 & 1.29 & 11.7 & 33.1 & 590 & 16.7 & 72.5 & 0.002 & 0.17 & 1.10 & & 2.10 & 207.0 & $\begin{array}{ll}0.91 \\
\end{array}$ & $<0.05$ & 111.8 & 464 & 0.41 & 2.9 & & & \begin{tabular}{|l|l|}
17.7 \\
\end{tabular} & & 87.2 & & 731 \\
\hline & 2.84 & 0.99 & 111.0 & 32.2 & 960 & 19.2 & 88.7 & $<0.002$ & 0.05 & 1.64 & & 2.30 & \begin{tabular}{|l|}
158.5 \\
\end{tabular} & \begin{tabular}{|l|l}
0.79 \\
\end{tabular} & 0.10 & $\begin{array}{l}9.4 \\
\end{array}$ & 0.434 & 0.61 & 3.6 & 172 & 1.5 & 20.0 & 106 & 90.1 & & $\overline{\mathrm{FAO}}$ \\
\hline 1 & 3.39 & 0.95 & 10.6 & 36.2 & 1080 & 19.6 & 85.2 & $<0.002$ & 0.05 & 1.77 & & 2.60 & 159.0 & \begin{tabular}{|c|}
0.81 \\
\end{tabular} & 0.10 & 9.3 & 0.425 & 0.59 & 4.1 & 189 & 1.4 & 20.8 & $12 \varepsilon$ & 85.9 & & 731 \\
\hline & 19 & 0.74 & 9.1 & 37.6 & 940 & 15.2 & 64.7 & $<0.002$ & 0.05 & 2.31 & & 1.90 & 136.0 & 0.67 & 0.11 & 8.0 & 0.454 & 0.51 & 4.2 & & & 23.3 & 12 & 79. & & \\
\hline & 2.86 & .74 & 11.0 & 46.8 & 1020 & 15.8 & 62.4 & $<0.002$ & 0.04 & 2.68 & & 1.80 & 143.0 & 0.80 & 0.10 & 8.1 & .683 & 0.57 & 4.3 & 222 & 1. & 24.2 & 18 & 92.4 & & FA04 \\
\hline 16 & 2.73 & 0.70 & 9.2 & 53.6 & 1240 & 14.4 & 62.5 & 0.002 & 0.04 & 2.48 & & 2.00 & 144.5 & $\begin{array}{l}0.67 \\
\end{array}$ & 0.09 & 7.9 & 0.599 & 0.59 & 4.2 & 21 & 1.1 & 29.0 & 202 & 74.8 & & 731 \\
\hline & & 1.12 & 9.7 & 25.1 & 750 & 14.6 & 63.2 & $<0.002$ & 0.03 & 1.29 & & 2.00 & 168.5 & \begin{tabular}{|l|l|}
0.73 \\
\end{tabular} & 0.07 & 8.2 & & 0.43 & 3.0 & & & 16.4 & 82 & 74 & & \\
\hline & 2.12 & 0.97 & 9.7 & 30.5 & 890 & 15.8 & 73.5 & $<0.002$ & 0.03 & 1.54 & & 2.00 & 155.5 & 0.74 & 0.08 & 8.6 & 0.402 & 0.46 & 3.3 & 16 & 1.2 & 18.7 & 106 & 80. & & 731 \\
\hline & 2.21 & 0.87 & 9.7 & 31.7 & 830 & 15.4 & 68.3 & $<0.002$ & 0.03 & 1.84 & & 2.00 & 145.5 & 0.74 & 0.09 & 8.4 & 0.416 & 0.46 & 3.3 & 169 & & 19.0 & 11 & 84. & & 731 \\
\hline & & & 8.7 & & 950 & 15.1 & & $<0.002$ & 0.04 & 1.64 & & & & 0.66 & 0.08 & 7.7 & & & & & & 27.7 & $3<$ & 74. & & \\
\hline 22 & 1.53 & 1.01 & 10.0 & 43.8 & 970 & 14.4 & 62.6 & $<0.002$ & 0.04 & 1.60 & & 1.80 & 177.0 & \begin{tabular}{|l|l|}
0.71 \\
\end{tabular} & 0.08 & 8.3 & 0.461 & 0.46 & 2.8 & 155 & 1.1 & 28.2 & 132 & 79.5 & & FA0403 \\
\hline 1323 & 0.91 & 0.93 & 8.7 & 29.8 & 850 & 16.8 & 62.5 & $<0.002$ & 0.06 & 1.31 & & 2.20 & 144.5 & 0.66 & 0.06 & 7.0 & 0.371 & 0.43 & 2.3 & 102 & 2.5 & 11.8 & 69 & 63.8 & & FA04C \\
\hline & & & 9.6 & & 580 & 14.4 & & $<0.002$ & 0.03 & 1.24 & & & & $\begin{array}{ll}0.72 \\
\end{array}$ & $<0.05$ & 7.7 & 0.447 & 0.42 & 2.0 & & & & & 62.9 & & \\
\hline 1325 & 0.84 & 1.37 & \begin{tabular}{|l|l|}
11.2 \\
\end{tabular} & 34.0 & 680 & 14.6 & 61.3 & $<0.002$ & 0.02 & 1.23 & 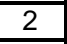 & 2.00 & \begin{tabular}{|c|}
187.0 \\
\end{tabular} & 0.85 & $<0.05$ & 9.5 & 0.591 & 0.39 & 2.2 & 139 & 1.6 & 16.2 & 96 & 73.2 & 30 & FA04 \\
\hline 1327 & 0.86 & 1.36 & 10.7 & 32.8 & 670 & 14.4 & 61.3 & $<0.002$ & 0.02 & 1.22 & & 1.90 & 192.0 & \begin{tabular}{|c|}
0.83 \\
\end{tabular} & 0.05 & 8.9 & 0.486 & 0.37 & 2.1 & 121 & 1.3 & 16.2 & 84 & 71.4 & & \\
\hline & 0.98 & 1.31 & 12.2 & 29.5 & 790 & 13.6 & 56.5 & $<0.002$ & 0.02 & 1.16 & & 1.90 & 210.0 & 0.93 & $<0.05$ & 12.8 & 0.545 & 0.34 & 2.7 & & & & & 94.2 & & \\
\hline 1329 & 0.83 & 1.39 & 9.7 & 29.2 & 770 & 13.2 & 61.4 & $<0.002$ & 0.03 & 1.26 & 1 & 1.70 & 218.0 & \begin{tabular}{|l|}
0.76 \\
\end{tabular} & $<0.05$ & 9.0 & 0.424 & 0.38 & 2.1 & 115 & 1.2 & 15.4 & 78 & 69.9 & 3 & 731 \\
\hline & 0.73 & 1.52 & 10.6 & 29.3 & 810 & 12.5 & 57.6 & $<0.002$ & 0.03 & 1.16 & & 1.80 & 242.0 & \begin{tabular}{|l|l|}
0.79 \\
\end{tabular} & $<0.05$ & 9.5 & 0.469 & 0.37 & 2.2 & & 1.2 & 17.1 & 16 & 75.9 & & \\
\hline 1331 & 0.52 & 1.52 & 13.7 & 22.2 & 1280 & 14.2 & 72.6 & $<0.002$ & 0.02 & 0.99 & 1 & 2.20 & 283.0 & \begin{tabular}{|c|}
0.99 \\
\end{tabular} & $<0.05$ & 12.8 & 0.418 & 0.44 & 3.5 & 112 & 1.3 & 16.8 & 71 & 59.4 & 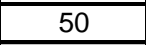 & FA04030731 \\
\hline 1332 & 0.94 & 1.66 & 29.7 & 24.7 & 2340 & 16.3 & 80.1 & $<0.002$ & 0.03 & 1.24 & $<$ & 3.60 & 339.0 & 2.35 & $<0.05$ & 36.2 & 0.754 & 0.48 & 6.7 & 179 & 1.7 & 23.3 & 122 & 63.5 & 50 & FA04030731 \\
\hline
\end{tabular}


Table 1. Location and New Trace Element Geochemical Results for 902 Stream-Sediment Samples collected in the Livengood Area, Livengood Quadrangle, Alaska IS = Insufficient Sample for Analysis

\begin{tabular}{|c|c|c|c|c|c|c|c|c|c|c|c|c|c|c|c|c|c|c|c|c|c|c|c|c|c|c|c|}
\hline MPLE & ME & $\mathrm{M} \mathrm{N}$ & $\begin{array}{l}\mathrm{Au} \\
\mathrm{ppm}\end{array}$ & $\begin{array}{c}\mathrm{Ag} \\
\mathrm{ppm}\end{array}$ & $\begin{array}{l}\text { Al } \\
\%\end{array}$ & $\begin{array}{l}\text { As } \\
\text { ppm }\end{array}$ & $\begin{array}{l}\mathrm{Ba}^{*} \\
\mathrm{ppm}\end{array}$ & $\begin{array}{c}\mathrm{Be} \\
\mathrm{ppm}\end{array}$ & $\begin{array}{c}\mathrm{Bi} \\
\mathrm{ppm}\end{array}$ & $\begin{array}{c}\mathrm{Ca} \\
\%\end{array}$ & $\begin{array}{l}\mathrm{Cd} \\
\mathrm{ppm}\end{array}$ & $\begin{array}{c}\mathrm{Ce} \\
\mathrm{ppm}\end{array}$ & $\begin{array}{c}\text { Co } \\
\text { ppm }\end{array}$ & $\begin{array}{l}\mathrm{Cr}^{*} \\
\mathrm{ppm}\end{array}$ & $\begin{array}{c}\text { Cs } \\
\text { ppm }\end{array}$ & $\begin{array}{c}\mathrm{Cu} \\
\mathrm{ppm}\end{array}$ & $\begin{array}{c}\mathrm{Fe} \\
\% \\
\end{array}$ & $\begin{array}{c}\mathrm{Ga} \\
\mathrm{ppm}\end{array}$ & $\begin{array}{c}\mathrm{Ge} \\
\mathrm{ppm}\end{array}$ & $\begin{array}{l}\mathrm{Hf} \\
\mathrm{ppm}\end{array}$ & $\begin{array}{l}\mathrm{Hg} \\
\mathrm{ppm}\end{array}$ & $\begin{array}{c}\text { In } \\
\mathrm{ppm}\end{array}$ & $\begin{array}{l}\mathrm{K} \\
\% \\
\end{array}$ & $\begin{array}{c}\mathrm{La} \\
\mathrm{ppm}\end{array}$ & \begin{tabular}{c|}
$\mathrm{Li}$ \\
$\mathrm{ppm}$
\end{tabular} & $\begin{array}{c}\mathrm{Mg} \\
\% \\
\end{array}$ & $\begin{array}{l}\mathrm{Mn} \\
\mathrm{ppm}\end{array}$ \\
\hline 33 & & & 0.005 & & & & 710 & 1.55 & 0.18 & 1.47 & 0.40 & & & 76 & & 18.8 & & & 0.14 & 1.9 & 0.31 & 0.051 & 1.16 & 48.7 & 25.5 & 0.96 & 1630 \\
\hline & & & & & & & & 1.39 & & & 0.25 & & & & & & & & & & & & 1.16 & 5.2 & 7.5 & & 715 \\
\hline & & & 006 & & & & 0 & 1.16 & & & 0.22 & & & & 5.04 & 16 & 79 & 4.45 & 0.13 & & 15 & 048 & 1.14 & 42.8 & 1.0 & & 710 \\
\hline & & & & & & & & & & & & & & & & & & & & & & & 1.04 & & & & 3120 \\
\hline & & & 012 & 26 & 21 & .6 & 60 & 1.12 & 18 & 76 & 0.18 & 2.1 & 7.7 & & 3.10 & 23.6 & .63 & 4.55 & 0.09 & 2.1 & 35 & 047 & 1.14 & 31.3 & 0.5 & 0.71 & 274 \\
\hline & & & 061 & 36 & & 7.4 & 90 & 1.01 & 18 & 62 & 0.17 & 35 & 5.4 & $\overline{73}$ & 3.44 & 23.5 & 1.94 & 2.50 & 0.09 & & 75 & 034 & 1.09 & 24.6 & 5.9 & 0.57 & 187 \\
\hline & & & & & & 3.0 & & 0.99 & & 70 & 0.24 & & & & & 18.2 & & & & & & & & & & & 13 \\
\hline & & & 010 & 0.26 & 98 & & 660 & 0.94 & 0.18 & 71 & 0.12 & & 6.4 & 77 & 2.80 & 23.2 & 4.51 & 4.15 & 0.12 & & 35 & 043 & 1.07 & 26.8 & 9.7 & & 210 \\
\hline & & & .005 & 0.15 & & 8.9 & 10 & 1.28 & 15 & 11 & 0.32 & & 10.4 & & 2.95 & 22.7 & 2.73 & 13.95 & 0.12 & 2. & 34 & .047 & 1.22 & 30.8 & 3.1 & 0.05 & 1050 \\
\hline & & & .029 & 24 & & 36.7 & 20 & 0.80 & 14 & 42 & 0.29 & 4. & 8.5 & & 1.90 & 27.1 & 8.77 & 8.29 & 0.18 & & 57 & & & 20.5 & 3.4 & & 150 \\
\hline & & & 014 & 0.63 & 6 & 8 & 20 & 1.26 & 19 & 14 & 0.41 & 7. & 15.3 & & 11.35 & 38.2 & 3.25 & 15.90 & 0.14 & & 48 & 055 & 1.12 & 29.3 & 39.2 & 1.01 & 38 \\
\hline & & & & 19 & & & & 1.25 & & 54 & & & & & 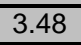 & 19. & 80 & 14.50 & 0.12 & & & 045 & 1.23 & 2.0 & 25.1 & & 83 \\
\hline & & & & 19 & & & & 27 & & 56 & & & & & $\overline{97}$ & & .23 & 4.40 & & & & & & 2.6 & 4.9 & & 06 \\
\hline & & & .005 & 0.14 & 8 & 7.3 & 30 & 1.25 & 13 & 51 & 0.24 & 61.0 & 2.4 & 81 & 2.65 & 17.6 & 2.92 & 14.65 & 0.12 & 1.8 & .21 & $\begin{array}{l}.045 \\
\end{array}$ & 1.28 & 30.6 & 24.2 & 01 & 62 \\
\hline & & & 005 & 15 & & & & & & & & & & & & & 2.98 & 14.30 & 0.13 & & & 046 & & & 3.1 & & 518 \\
\hline & & & 019 & .17 & & & 0 & .27 & 13 & 50 & 0. & 59.7 & 3.8 & 76 & 2.52 & & 4.72 & 13.3 & & & 52 & .044 & & 28.6 & 0.9 & & 2180 \\
\hline & & & 26 & .34 & & 16.0 & 50 & 1.23 & 54 & 12 & 1.86 & 55.1 & $7 . \varepsilon$ & & & 41. & 3.23 & 6.70 & 0.13 & & & 36 & & 1.7 & 0.2 & & 180 \\
\hline & & & & 0.34 & & & & .08 & & & 0.30 & & & & & & & 5.40 & & & & | & & & 4. & & 553 \\
\hline & & & 014 & 23 & 29 & 5. & 20 & 1.11 & 62 & .12 & 0.47 & 3.1 & & 91 & 3.58 & 18.6 & 2.55 & 3.7 & 13 & & 17 & 7 & 1.12 & 30.5 & 0.7 & 0.9 & 477 \\
\hline & & & & 22 & & & & & & & & & & & & 22. & 6 & 5.2 & 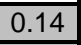 & & & & & 0.0 & 4.4 & & 490 \\
\hline & & & & 0.25 & & & & 1.12 & & & 0.55 & & & & 3.17 & & 2.71 & & & & & & & 30.3 & & & 580 \\
\hline & & & 012 & 0.17 & & & 40 & 1.13 & 0.16 & .44 & 0.34 & 70.2 & 2.8 & & 2.89 & 19.8 & 2.88 & 14.55 & 0.14 & & D.38 & $\begin{array}{l}.046 \\
\end{array}$ & 1.23 & 34.9 & 4.5 & 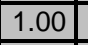 & 516 \\
\hline & & & 116 & 22 & & & & 1.20 & & & 0.51 & & & & 0 & 24 & .32 & 5.20 & 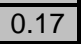 & & & 054 & & 33.6 & 4. & & 560 \\
\hline & & & & & & & & & & & & & & & & & & & & & & & & .3 & 34.1 & & 894 \\
\hline & & & & 17 & & & & .14 & & & & & & & 84 & 16. & 2.90 & 14.65 & 0.1 & & 2 & .050 & 1.1 & 37.6 & 31.6 & 10 & 797 \\
\hline & & & & 19 & & & & & & & & & & & & & & 4.4 & & & & 3 & & 1.8 & $\overline{9}$ & & 15 \\
\hline & & & & 0.14 & & & & 1.32 & & & 0.32 & 69 & & & 2.71 & 20. & 2.99 & 14.85 & & & & 3 & & 5.1 & 5 & & 04 \\
\hline & & & 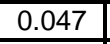 & 36 & & & & & & & & & & & & 22. & & 4. & & & & & & 25.0 & 3.7 & & 090 \\
\hline & & & & 0.85 & & & & & & & & & & & & & & & & & & & & & 1.5 & & 1230 \\
\hline & & & & & & & & & & & & & & & & & & & & & & & & 308 & & & 854 \\
\hline & & & 297 & .67 & & 188.5 & & 21 & & & & & & & & 34 & & 5 & & & & & & 1.8 & 5.8 & & 015 \\
\hline & & & & .69 & & & & & & & & & & & & & & & & & & & & .5 & 8 & & 560 \\
\hline & & & & & & & & & & & & & & & & 37. & & & & & & & & & 77 & & 185 \\
\hline & & 58333 & 0.013 & 0.40 & 8 & 111.8 & & 1.15 & 22 & 42 & 0.52 & & 14.5 & 96 & 4.00 & 19.7 & 3.08 & 14.60 & 0.16 & & 54 & 075 & 1.27 & 34.0 & \begin{tabular}{l|l|}
5.4 \\
\end{tabular} & & 1200 \\
\hline & & & & 0.73 & & & & & & & & & & & & 30. & & & & & & & & & 0. & & 889 \\
\hline & & & .005 & 0.59 & & & & & & & & & & & & & & & & & & .054 & & 35.7 & 6. & & 754 \\
\hline & & & 0.035 & 0.65 & & & & 1.13 & & 0.98 & 0.91 & & 18. & & & 28. & & 14.70 & & & & 081 & 1.12 & 31.0 & 29.0 & \begin{tabular}{|l|l|}
0.92 \\
\end{tabular} & 1050 \\
\hline & & & & & & & & & & & & & & & & & & & & & & & & & \begin{tabular}{l|l}
35.2 \\
\end{tabular} & & 893 \\
\hline & & & 0.025 & 0.83 & & & & 1.10 & & & 2. & & & & & 30.4 & 2.59 & & & & & .054 & 12 & 30.1 & $\begin{array}{l}6.3 \\
\end{array}$ & & 040 \\
\hline & & & 0.021 & 0.81 & & 14 & 69 & 1.1 & & & 1.68 & & 14 & 88 & & 32.0 & & 14.20 & 0.17 & 1. & & 049 & & 31.9 & 35.3 & & 500 \\
\hline & & & & & & & & & & & & & & & & & & & & & & & & 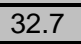 & 3601 & & 000 \\
\hline & & 7250 & 0.024 & 1.17 & 5.52 & 17.8 & 650 & 1.12 & 0.1 & 1.3 & 3.17 & 59.5 & 13. & 78 & 4. & 28.5 & 2.5 & 13.6 & 0.17 & 1.6 & & & 06 & 28.1 & 40.3 & & 853 \\
\hline & & & 0.089 & 0.00 & & 13.4 & & & $0.1<$ & & & & & & & $\angle 2.0$ & & & ניז. & & & & & $\angle 8.1$ & 30.1 & & 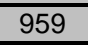 \\
\hline
\end{tabular}


Table 1. Location and New Trace Element Geochemical Results for 902 Stream-Sediment Samples collected in the Livengood Area, Livengood Quadrangle, Alaska IS = Insufficient Sample for Analysis

\begin{tabular}{|c|c|c|c|c|c|c|c|c|c|c|c|c|c|c|c|c|c|c|c|c|c|c|c|c|c|c|}
\hline AMPLE & $\begin{array}{l}\text { Mo } \\
\text { ppm }\end{array}$ & $\begin{array}{c}\mathrm{Na} \\
\% \\
\end{array}$ & $\begin{array}{l}\mathrm{Nb} \\
\mathrm{ppm}\end{array}$ & $\begin{array}{r}\mathrm{Ni} \\
\mathrm{ppm}\end{array}$ & $\begin{array}{c}\mathrm{P} \\
\mathrm{ppm}\end{array}$ & $\begin{array}{c}\mathrm{Pb} \\
\mathrm{ppm}\end{array}$ & $\begin{array}{l}\mathrm{Rb} \\
\mathrm{ppm}\end{array}$ & $\begin{array}{c}\mathrm{Re} \\
\mathrm{ppm}\end{array}$ & $\begin{array}{l}\mathrm{S} \\
\%\end{array}$ & $\begin{array}{c}\text { Sb } \\
\mathrm{ppm}\end{array}$ & $\begin{array}{c}\text { Se } \\
\mathrm{ppm}\end{array}$ & $\begin{array}{l}S^{S n^{*}} \\
\text { ppm }\end{array}$ & $\begin{array}{c}\mathrm{Sr} \\
\mathrm{ppm}\end{array}$ & $\begin{array}{l}\mathrm{Ta}^{*} \\
\mathrm{ppm}\end{array}$ & $\begin{array}{c}\text { Te } \\
\text { ppm }\end{array}$ & $\begin{array}{c}\text { Th } \\
\text { ppm }\end{array}$ & $\begin{array}{l}\mathrm{Ti}^{\star} \\
\%\end{array}$ & \begin{tabular}{c|}
$\mathrm{Tl}$ \\
$\mathrm{ppm}$
\end{tabular} & $\begin{array}{c}\mathrm{U} \\
\mathrm{ppm}\end{array}$ & \begin{tabular}{|c|}
$\mathrm{V}$ \\
$\mathrm{ppm}$
\end{tabular} & $\begin{array}{l}W^{*} \\
\mathrm{ppm}\end{array}$ & \begin{tabular}{|c|}
$\mathrm{Y}$ \\
$\mathrm{ppm}$ \\
\end{tabular} & $\begin{array}{l}\mathrm{Zn} \\
\mathrm{ppm}\end{array}$ & $\begin{array}{l}\mathrm{Zr}^{\star} \\
\mathrm{ppm}\end{array}$ & $\begin{array}{c}\text { Pulp Wt } \\
\text { grams }\end{array}$ & $\begin{array}{c}\text { Lab } \\
\text { Report }\end{array}$ \\
\hline 1333 & 1.14 & 1.32 & 16.6 & 27.5 & 1300 & 16.1 & 64.9 & $<0.002$ & 0.03 & 1.26 & 2 & 2.30 & 240.0 & 1.27 & $<0.05$ & 11.2 & 0.457 & 0.40 & 3.2 & 121 & 1.3 & 19.2 & 85 & 65.3 & 50 & FA04030731 \\
\hline 1335 & 0.66 & .52 & 13.7 & 26.8 & 980 & 16.1 & 58.1 & $<0.002$ & 0.02 & 1.26 & 1 & 2.10 & 240.0 & 1.05 & $<0.05$ & 12.3 & 0.493 & 0.35 & 2.8 & 113 & 1.5 & 17.4 & 75 & 67.8 & 38 & A04030731 \\
\hline 1336 & 0.98 & 1.21 & 11.7 & 27.7 & 710 & 16.6 & 60.6 & $<0.002$ & 0.03 & 1.16 & & 2.00 & 186.5 & 0.97 & $<0.05$ & 9.9 & 0.454 & 0.38 & 2.0 & 112 & 1.2 & 14.1 & 79 & 60.6 & 27 & A04030731 \\
\hline & 1.90 & $\overline{09}$ & 9.9 & 28.0 & 1230 & 15.2 & 57.5 & $<0,002$ & 0.05 & 1.31 & & 1.80 & 203.0 & 0.72 & 0.05 & 10.2 & 0.354 & 0.37 & 2.9 & 125 & 910 & 18.7 & 9 & 586 & & A04030731 \\
\hline 1428 & 2.10 & 0.91 & 9.1 & 22.6 & 700 & 13.4 & 63.7 & $<0.002$ & 0.03 & 1.32 & 2 & 2.00 & 144.0 & 0.68 & 0.07 & 8.0 & 0.389 & 0.43 & 3.2 & 147 & 1.1 & 13.2 & 51 & $\overline{71.8}$ & 23 & FA04030731 \\
\hline 1429 & 2.06 & 0.71 & 6.3 & 18.6 & 810 & 12.5 & 62.6 & 0.003 & 0.05 & 1.20 & 2 & 1.80 & 122.5 & $\begin{array}{ll}0.47 \\
\end{array}$ & 0.08 & 6.2 & 0.290 & 0.39 & 3.0 & 146 & 0.8 & 11.9 & 40 & 58.1 & 18 & FA04030731 \\
\hline 1430 & 1.81 & .80 & 7.6 & 21.4 & 880 & 11.2 & 57.0 & 0.002 & 0.05 & 1.06 & 2 & 1.60 & 131.0 & 0.57 & 0.06 & 6.7 & 0.321 & 0.37 & 2.6 & 112 & 1.0 & \begin{tabular}{|l|}
12.8 \\
\end{tabular} & 47 & 64.4 & & FA04030731 \\
\hline 1431 & 1.16 & 0.91 & 8.4 & 20.5 & 610 & 13.0 & 60.7 & $<0.002$ & 0.22 & 1.20 & & 1.70 & 142.0 & 0.65 & 0.05 & 7.8 & 0.367 & 0.37 & 2.4 & 114 & 1.1 & 11.4 & 45 & 68.8 & 5 & FA04030731 \\
\hline & 1.35 & .13 & 9.7 & 32.9 & 680 & 13.8 & 61.3 & $<0.002$ & 0.03 & 1.17 & & 3.60 & 180.0 & 0.73 & 0.05 & 8.3 & 0.403 & 0.37 & 2.4 & 113 & 1.0 & 16.1 & & 73.6 & & FA04030731 \\
\hline 1435 & 2.06 & 0.41 & 4.4 & 26.6 & 1130 & 10.4 & 39.0 & 0.002 & 0.13 & 1.20 & 3 & 1.10 & 78.3 & 0.33 & 0.09 & 5.4 & 0.190 & 0.25 & 2.1 & 100 & 0.7 & 15.8 & 48 & 45.0 & 21 & FA04030731 \\
\hline 1436 & 1.09 & 0.87 & 7.7 & 42.1 & 960 & 15.2 & 70.6 & $<0.002$ & 0.07 & 1.02 & 2 & 2.70 & 159.5 & \begin{tabular}{|l|}
0.57 \\
\end{tabular} & 0.06 & 7.0 & 0.368 & 0.51 & 1.9 & 142 & 1.0 & \begin{tabular}{|l|}
21.7 \\
\end{tabular} & 94 & 64.5 & 1 & FA04030731 \\
\hline & 0.61 & 35 & 9.2 & 31.0 & 770 & 2.4 & 60.5 & $<0.002$ & 0.04 & 1.02 & & 1.70 & 224.0 & 0.69 & $<0.05$ & 8.2 & 0.405 & 0.37 & 2.0 & 115 & 1.0 & 17.5 & 74 & 66.7 & & \\
\hline $143 !$ & 0.78 & 1.50 & 11.8 & 32.2 & 740 & 11.7 & 53.8 & $<0.002$ & 0.02 & 1.06 & 2 & 1.80 & 220.0 & 0.91 & $<0.05$ & 11.2 & 0.568 & 0.33 & 2.4 & 131 & 1.3 & 18.9 & 73 & 87.4 & & FA04030731 \\
\hline $144 C$ & 0.72 & 1.51 & 9.5 & 29.7 & 660 & 11.6 & 59.3 & $<0.002$ & 0.02 & 1.02 & 1 & 1.80 & 227.0 & 0.73 & $<0.05$ & 7.9 & 0.416 & 0.37 & 1.9 & 115 & 1.0 & 14.8 & 70 & 64.9 & 4 & FA04030731 \\
\hline 1441 & 0.80 & 1.43 & 9.9 & 30.1 & 660 & 12.0 & 57.5 & $<0.002$ & 0.03 & 1.10 & & 1.80 & 220.0 & 0.75 & $<0.05$ & 8.5 & 0.432 & 0.34 & 2.2 & 117 & 1.2 & \begin{tabular}{|l|}
15.8 \\
\end{tabular} & 68 & 69.4 & & \\
\hline 1442 & 1.00 & 1.25 & 8.4 & 33.0 & 880 & 11.0 & 58.0 & $<0.002$ & 0.05 & 1.22 & & 1.70 & 213.0 & \begin{tabular}{|l|l|}
0.67 \\
\end{tabular} & 0.05 & 7.6 & 0.356 & 0.35 & 1.9 & 111 & 1.0 & \begin{tabular}{|l|}
16.8 \\
\end{tabular} & 76 & 63.5 & 2 & FA04030731 \\
\hline & 1.09 & 1.02 & 9.3 & 37.3 & 690 & 29.4 & 78.3 & $<0.002$ & 0.04 & 1.28 & & 2.60 & 186.5 & 0.73 & 0.07 & 7.7 & 0.409 & 0.58 & 2.1 & 134 & 1.2 & \begin{tabular}{|l|}
17.2 \\
\end{tabular} & 126 & 62. & & FA04030731 \\
\hline 144 & 0.82 & 1.26 & 9.9 & 29.8 & 600 & 17.0 & 69.2 & $<0.002$ & 0.02 & 1.02 & 2 & 2.80 & 202.0 & 0.80 & 0.05 & 9.5 & 0.467 & 0.47 & 2.2 & 129 & 1.8 & 15.4 & 81 & 71 & & FA04030731 \\
\hline 1447 & 0.59 & 1.21 & 9.9 & 25.8 & 480 & 14.7 & 55.2 & $<0.002$ & 0.02 & 0.88 & 1 & 2.30 & 176.5 & \begin{tabular}{|l|l|}
0.77 \\
\end{tabular} & $<0.05$ & 7.5 & 0.465 & 0.39 & 1.8 & 116 & 1.7 & 13.6 & 76 & 65.7 & 2 & FA04030731 \\
\hline 1448 & 0.74 & 1.36 & 10.4 & 31.9 & 640 & 15.0 & 64.9 & $<0.002$ & 0.02 & 1.28 & & 2.00 & 211.0 & 0.83 & \begin{tabular}{|l|}
0.05 \\
\end{tabular} & 8.6 & 0.439 & 0.41 & 2.1 & 115 & 1.5 & 15.8 & 81 & 68.9 & & FA04030731 \\
\hline $144 \mathrm{C}$ & 0.70 & 1.29 & 9.5 & 30.0 & 640 & 15.2 & 64.3 & $<0.002$ & 0.02 & 1.14 & & 2.00 & 201.0 & 0.77 & $<0.05$ & 8.4 & 0.409 & 0.41 & 2.0 & 11 & 1. & 14.7 & 8 & 63 & & \\
\hline 1450 & 0.69 & 1.40 & 10.4 & 31.0 & 670 & 12.8 & 58.5 & $<0.002$ & 0.03 & 1.18 & & 1.90 & 212.0 & 0.80 & $<0.05$ & 9.1 & 0.450 & 0.36 & 2.1 & 116 & 1.2 & 16.1 & 76 & 68.2 & 4 & FA04030731 \\
\hline 145 & 0.79 & 1.34 & 10.2 & 35.8 & 730 & 19.3 & 64.7 & $<0.002$ & 0.03 & 2.14 & & 3.00 & 187.0 & 0.79 & 0.05 & 8.2 & 0.448 & 0.43 & 2.0 & 126 & 1.2 & 17.0 & 10 & 67.0 & & FA04030731 \\
\hline 145 & 0.75 & .35 & 10.0 & 35.6 & 670 & 15.8 & 64.4 & $<0.002$ & 0.03 & 1.66 & 2 & 1.90 & 188.0 & \begin{tabular}{|l|l|}
0.77 \\
\end{tabular} & 0.05 & 8.7 & 0.458 & 0.40 & 2.0 & 127 & 1.2 & 16.4 & 88 & 69 . & & 7731 \\
\hline 145 & 1.16 & 1.34 & 11.0 & 31.5 & 640 & 15.2 & 59.9 & $<0.002$ & 0.02 & 1.36 & & 1.90 & 186.5 & 0.90 & $<0.05$ & 9.5 & 0.489 & 0.36 & 2.2 & 123 & 2.0 & \begin{tabular}{|l|}
16.5 \\
\end{tabular} & 82 & 74.4 & 4 & 30731 \\
\hline 14 & 0.73 & 1.27 & 9.6 & 32.3 & 610 & 15.9 & 60.3 & $<0.002$ & 0.03 & 1.41 & 2 & 1.90 & 185.5 & 0.73 & $<0.05$ & 7.8 & 0.429 & 0.37 & 1.9 & 117 & 1. & \begin{tabular}{|l|}
15.8 \\
\end{tabular} & 8 & 64.5 & & FA04030731 \\
\hline 1461 & 0.70 & 1.47 & 10.4 & 30.9 & 690 & 12.8 & 61.2 & $<0.002$ & 0.03 & 1.43 & & 1.80 & 228.0 & 0.82 & $<0.05$ & 9.2 & 0.441 & 0.36 & 2.2 & 116 & 1.3 & 16.8 . & 7 & 70. & & FA04030731 \\
\hline 1463 & 0.88 & 1.13 & 8.3 & 29.7 & 670 & 19.8 & 66.3 & $<0.002$ & 0.04 & 1.07 & & 3.30 & 157.0 & 0.63 & \begin{tabular}{|l|}
0.05 \\
\end{tabular} & 6.3 & 0.394 & 0.45 & 1.7 & 128 & 1.3 & 13.0 & 7 & 63.1 & & FA04030731 \\
\hline & 0. & 1.01 & 7.4 & 36.1 & 760 & 28.5 & 87.6 & $<0.002$ & 0.06 & 2.36 & & 11.30 & 138.5 & \begin{tabular}{|l|}
0.57 \\
\end{tabular} & 0.0 & 6.2 & 0.373 & 0.62 & 1.5 & 141 & 8.2 & 15.6 & 94 & 54 & & \\
\hline 1465 & 1.04 & 1.03 & 8.2 & 42.0 & 900 & 51.2 & 81.7 & $<0.002$ & 0.06 & 2.06 & & 2.90 & 153.0 & 0.65 & 0.07 & 6.5 & 0.398 & 0.60 & 1.9 & 149 & 1.5 & \begin{tabular}{|l|}
24.5 \\
\end{tabular} & 126 & 67. & & FA04030731 \\
\hline 1466 & 0.98 & 1.05 & 8.6 & 32.8 & 710 & 26.5 & 93.8 & $<0.002$ & 0.06 & 2.40 & 2 & 19.80 & 148.5 & 0.65 & 0.08 & 7.3 & 0.419 & 0.65 & 1.9 & 141 & 36.5 & \begin{tabular}{|l|}
14.1 \\
\end{tabular} & 78 & 71.5 & & FA04030731 \\
\hline & & 1.18 & 9.0 & 32.9 & 590 & 40.4 & 72.4 & $<0.002$ & 0.03 & 2.29 & & 3.90 & 149.5 & 0.68 & 0.05 & 7.0 & 436 & 0.54 & 1.9 & 135 & 1.3 & 14.6 & 103 & 61.4 & & \\
\hline 1469 & 0.98 & 1.02 & 8.6 & 35.2 & 700 & 26.8 & 96.7 & $<0.002$ & 0.06 & 2.59 & & 22.50 & 138.5 & 0.65 & 0.07 & 6.9 & 0.424 & 0.64 & 1.7 & 147 & 45.8 & 13.3 & 83 & 63.9 & 3 & FA04030731 \\
\hline 1470 & 0.81 & 1.36 & 8.8 & 32.6 & 710 & 54.4 & 66.5 & $<0.002$ & 0.03 & 2.20 & & 2.50 & 205.0 & 0.67 & $<0.05$ & 8.5 & 0.412 & 0.43 & 2.0 & 122 & 1.0 & 16.3 & 105 & 65.7 & 3 & FA04030731 \\
\hline & & 1.00 & 8.8 & 35.7 & 960 & & 64.5 & & 006 & 1.66 & & 1.80 & 167.0 & 0.66 & $<0.05$ & 7.6 & 0.361 & 0.43 & 2.1 & 109 & & 21.7 & & & & FA04030731 \\
\hline 1472 & 1.07 & 1.07 & 10.0 & 36.6 & 790 & 16.4 & 71.3 & $<0.002$ & 0.06 & 2.45 & 2 & 2.00 & 190.5 & 0.75 & \begin{tabular}{|l|}
0.06 \\
\end{tabular} & 8.2 & 0.418 & 0.46 & 2.4 & 125 & 1.1 & 21.3 & 99 & 63.2 & 2 & FA04030731 \\
\hline 1473 & 0.78 & 1.05 & 7.8 & 31.9 & 710 & 17.2 & 69.8 & $<0.002$ & 0.05 & 1.29 & & 2.50 & 157.0 & \begin{tabular}{|l|}
0.61 \\
\end{tabular} & 0.05 & 7.7 & 0.347 & 0.42 & 2.0 & 109 & 1.3 & \begin{tabular}{|l|}
15.7 \\
\end{tabular} & 94 & 58.0 & 1 & A04030731 \\
\hline & 0.73 & 1.05 & 8.9 & 34.9 & 680 & 20.3 & 62.3 & & & 2.09 & & 1.90 & 210.0 & 0.68 & & 7.1 & 0.382 & 0.41 & & 108 & & \begin{tabular}{|l|}
16.8 \\
\end{tabular} & 108 & 57.0 & & \\
\hline 1477 & 0.68 & 1.06 & 8.7 & 33.0 & 820 & 32.2 & 63.3 & $<0.002$ & 0.16 & 2.23 & 2 & 2.10 & 228.0 & \begin{tabular}{|l|l|}
0.68 \\
\end{tabular} & $<0.05$ & 7.7 & 0.399 & 0.42 & 2.0 & 108 & 1.5 & \begin{tabular}{|l|}
16.4 \\
\end{tabular} & 126 & 60.5 & 10 & FA04030731 \\
\hline 1478 & 0.65 & 1.17 & 9.5 & 33.4 & 670 & 37.8 & 65.0 & $<0.002$ & 0.09 & 2.35 & & 2.10 & 212.0 & 0.75 & \begin{tabular}{|l|}
0.05 \\
\end{tabular} & 8.0 & 0.434 & 0.49 & 2.1 & 114 & 2.5 & \begin{tabular}{|l|}
16.3 \\
\end{tabular} & 130 & 61.4 & 24 & A04030731 \\
\hline & 1.26 & 1.06 & 9.5 & 69.3 & 740 & 43.4 & 72.0 & & 0.07 & 4.04 & 2 & 1.90 & 194.5 & & 0.07 & 8.2 & 0.454 & 1.17 & 2.1 & & 6. & \begin{tabular}{|l|}
15.8 \\
\end{tabular} & 558 & 63 & & FA04030731 \\
\hline 1481 & 0.58 & 1.07 & 8.0 & 33.2 & 680 & 44.5 & 55.5 & $<0.002$ & 0.12 & 4.98 & 3 & 2.00 & 238.0 & 0.62 & 0.05 & 7.2 & 0.375 & 0.43 & 1.7 & 107 & 1.0 & 16.0 & 182 & 56.3 & 20 & FA04030731 \\
\hline 1482 & 0.62 & 1.05 & 8.3 & 30.4 & 600 & 23.3 & 49.1 & $<0.002$ & 0.13 & 2.25 & 3 & 1.90 & 233.0 & 0.62 & $<0.05$ & 7.0 & 0.381 & 0.36 & 1.8 & 104 & 1.1 & \begin{tabular}{|l|l|}
13.8 \\
\end{tabular} & 132 & 52.6 & 18 & FA04030731 \\
\hline
\end{tabular}


Table 1. Location and New Trace Element Geochemical Results for 902 Stream-Sediment Samples collected in the Livengood Area, Livengood Quadrangle, Alaska IS = Insufficient Sample for Analysis

\begin{tabular}{|c|c|c|c|c|c|c|c|c|c|c|c|c|c|c|c|c|c|c|c|c|c|c|c|c|c|c|c|}
\hline MPLE & TM E & $\mathrm{M} N$ & $\begin{array}{c}\mathrm{Au} \\
\mathrm{ppm}\end{array}$ & \begin{tabular}{|l|}
$\mathrm{Ag}$ \\
$\mathrm{ppm}$
\end{tabular} & $\begin{array}{l}\text { Al } \\
\%\end{array}$ & $\begin{array}{l}\text { As } \\
\text { ppm }\end{array}$ & $\begin{array}{l}\mathrm{Ba}^{*} \\
\mathrm{ppm}\end{array}$ & \begin{tabular}{|c|}
$\mathrm{Be}$ \\
$\mathrm{ppm}$
\end{tabular} & $\begin{array}{c}\mathrm{Bi} \\
\mathrm{ppm}\end{array}$ & $\begin{array}{c}\mathrm{Ca} \\
\%\end{array}$ & $\begin{array}{c}\mathrm{Cd} \\
\mathrm{ppm}\end{array}$ & $\begin{array}{l}\mathrm{Ce} \\
\mathrm{ppm}\end{array}$ & $\begin{array}{l}\text { Co } \\
\text { ppm }\end{array}$ & $\begin{array}{l}\mathrm{Cr}^{\star} \\
\mathrm{ppm}\end{array}$ & $\begin{array}{c}\text { Cs } \\
\mathrm{ppm}\end{array}$ & $\begin{array}{c}\mathrm{Cu} \\
\mathrm{ppm}\end{array}$ & $\begin{array}{c}\mathrm{Fe} \\
\%\end{array}$ & $\begin{array}{c}\mathrm{Ga} \\
\mathrm{ppm}\end{array}$ & $\begin{array}{c}\text { Ge } \\
\mathrm{ppm}\end{array}$ & $\begin{array}{c}\mathrm{Hf} \\
\mathrm{ppm}\end{array}$ & $\begin{array}{l}\mathrm{Hg} \\
\mathrm{ppm}\end{array}$ & $\begin{array}{c}\text { In } \\
\text { ppm }\end{array}$ & $\begin{array}{l}\mathrm{K} \\
\%\end{array}$ & $\begin{array}{c}\mathrm{La} \\
\mathrm{ppm}\end{array}$ & \begin{tabular}{|c|}
$\mathrm{Li}$ \\
$\mathrm{ppm}$
\end{tabular} & $\begin{array}{c}\mathrm{Mg} \\
\%\end{array}$ & $\begin{array}{l}\mathrm{Mn} \\
\mathrm{ppm}\end{array}$ \\
\hline 483 & 0056 & 50675 & 0.012 & & & & 620 & & & & & & & 83 & & 27.8 & 2.54 & & 0.15 & \begin{tabular}{|l|}
1.6 \\
\end{tabular} & 0.33 & 0.058 & \begin{tabular}{|l|l|}
1.14 \\
\end{tabular} & 29.7 & \begin{tabular}{|l|}
40.5 \\
\end{tabular} & 1.00 & 869 \\
\hline & & & & & & & 30 & & & & & & & & & & & & & & & & 1.34 & 28.3 & \begin{tabular}{|l|l}
33.8 \\
\end{tabular} & 0.95 & \\
\hline & & & & & & & 750 & & & & & & & & & 25.1 & & & 0.16 & & & 0.055 & 1.12 & 25.5 & 34.9 & 0.88 & 100 \\
\hline & & & & 52 & & & 840 & & & & & & & & & & & & & & & & & 28.2 & & & 160 \\
\hline & & & 045 & 0.43 & .58 & 6.6 & 710 & .23 & 15 & 1.43 & \begin{tabular}{|l|}
1.38 \\
\end{tabular} & 8.9 & 3.4 & & 6 & 19.7 & 2.64 & 4.55 & 0.15 & 1.7 & 0.27 & 0.058 & 1.18 & 28.3 & 33.4 & 0.93 & 495 \\
\hline & & & 011 & \begin{tabular}{|l|l|}
0.26 \\
\end{tabular} & 71 & 3.2 & 710 & 22 & 16 & 1.49 & \begin{tabular}{|l|}
0.69 \\
\end{tabular} & 5.0 & 2. & & $\overline{0}$ & 20.4 & 2.83 & 4.30 & 0.17 & 1.8 & 35 & 0.046 & 1.24 & 32.2 & 28.2 & 0.98 & 326 \\
\hline & & & & 34 & & & 740 & & & & & & & & & & 283 & & & & & & & 27.4 & 31.1 & & 98 \\
\hline & & & 016 & 28 & 50 & & 940 & & & 86 & 0.51 & & 5.1 & & & $\overline{99.4}$ & & & 0.21 & & & .046 & 1.05 & 30.1 & 21.5 & 0.072 & 992 \\
\hline & & & 010 & 0.22 & 37 & & 860 & 22 & $\overline{18}$ & 0.88 & 0.44 & 58.6 & 15.8 & & 2.70 & 42.6 & 3.18 & 13.30 & 0.19 & 1.9 & 0.21 & 0.044 & 1.08 & 28.3 & 20.6 & 0.70 & 1135 \\
\hline & & & .007 & 0.28 & & 2.6 & 1020 & 34 & 14 & 1.26 & 1.31 & & & & & & 181 & 15.30 & & 2.1 & & 0.055 & 0.99 & 25.1 & \begin{tabular}{|l|}
19.1 \\
\end{tabular} & \begin{tabular}{|l}
1.18 \\
\end{tabular} & 1125 \\
\hline & & & 0.011 & 0.21 & 5.70 & 10.5 & 900 & 1.28 & 13 & 1.62 & 1.28 & 61.3 & 23. & & 2.3 & 48.4 & 4.48 & 4.85 & 0.23 & 2. & 0.17 & \begin{tabular}{|l|l|}
0.054 \\
\end{tabular} & 1.00 & 28.7 & 21.5 & 1.12 & 235 \\
\hline & & & IS & 0.18 & 02 & 9.2 & 980 & 13 & & 42 & \begin{tabular}{|l|}
2.02 \\
\end{tabular} & & 2. & & & 5. & 3.03 & 2.10 & 0.20 & & & \begin{tabular}{|l|l|}
0.043 \\
\end{tabular} & 0.92 & 27.3 & \begin{tabular}{|l|l|}
20.8 \\
\end{tabular} & 0.12 & $46 \mathrm{C}$ \\
\hline & & & 0.005 & 17 & & 5.7 & 50 & & & 42 & \begin{tabular}{|l|}
1.01 \\
\end{tabular} & & 0 & & 5 & & 38 & 4.30 & 0.22 & & 0.17 & .049 & & 28.8 & 11.9 & & 310 \\
\hline & & & 010 & \begin{tabular}{|l|l|}
0.14 \\
\end{tabular} & .47 & 9.7 & 910 & 1.26 & 12 & 1.40 & \begin{tabular}{|l|l|}
0.87 \\
\end{tabular} & 55.0 & 17.8 & & 2.27 & 41.2 & 3.54 & 4.25 & 0.19 & 1.9 & 16 & 0.048 & \begin{tabular}{|l|l}
1.01 \\
\end{tabular} & 26.0 & 20.8 & \begin{tabular}{|l|l|} 
\\
\end{tabular} & 889 \\
\hline & & & 009 & & & & & & & & & 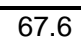 & & & & & & 5.70 & 0.22 & & & 0.055 & & 31.5 & \begin{tabular}{|l|}
23.9 \\
\end{tabular} & & 1790 \\
\hline & & & 0.010 & .08 & 18 & 6.4 & 660 & 1.10 & 09 & 1.64 & \begin{tabular}{|l|l|}
0.19 \\
\end{tabular} & 104.0 & 6 & & $\sqrt{9}$ & 14. & 308 & 2.20 & 0.23 & 2. & & .036 & \begin{tabular}{|l}
0.97 \\
\end{tabular} & 50.0 & 1.0 & & 70 \\
\hline & & & 006 & \begin{tabular}{|l|l|}
0.10 \\
\end{tabular} & 28 & & 710 & .13 & & 47 & 0.23 & 7.3 & 7.8 & & & 19 & 3.07 & 2.40 & 0.22 & 1. & & .036 & 1. & 36.4 & 23.5 & 1.68 & 338 \\
\hline & & & 007 & 08 & & & & & & & & & & & & & & & & & & 35 & & 30.2 & 25.4 & & 15 \\
\hline & & & $\overline{005}$ & \begin{tabular}{|l|l|}
0.11 \\
\end{tabular} & 29 & 1.8 & 770 & 1.20 & 10 & 1.22 & 0.25 & 57.7 & 16. & 27 & 2.5 & & 3.02 & 2.60 & 0.18 & 1. & & .038 & 1.14 & 28.4 & 27.9 & 1.5 & $\overline{484}$ \\
\hline & & & .005 & 12 & & & & & & 1.26 & & 5 & & & & & & & 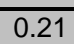 & & & $\overline{6}$ & 0.7 & 22.6 & 22.0 & 1.8 & 115 \\
\hline & & & .005 & \begin{tabular}{|l|l|}
0.11 \\
\end{tabular} & & 9 & 680 & 1.02 & & 1.50 & & 49.0 & 24.1 & & & 20. & 258 & & & & & .036 & & 23.6 & & & 337 \\
\hline & & & 005 & \begin{tabular}{|l|l|} 
\\
\end{tabular} & 5.62 & $\overline{6.4}$ & 820 & 1.27 & 12 & 1.42 & \begin{tabular}{|l|}
0.28 \\
\end{tabular} & 68.5 & 11.8 & 11 & 2.7 & 21. & 3.14 & 3.10 & 0.19 & 2. & .07 & .042 & 1.18 & 32.9 & 26.5 & 1.0 & 508 \\
\hline & & & .005 & \begin{tabular}{|l|l|}
0.19 \\
\end{tabular} & 98 & & & & & 86 & 0.82 & & & & & & 3.1 & 5.45 & 0.1 & & & .045 & 1. & 35.2 & 29.1 & 1. & 559 \\
\hline & & & 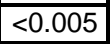 & & & 10.1 & 8 & & & & 1.06 & & & & & & & & & & & & & 38.8 & \begin{tabular}{|l}
30.3 \\
\end{tabular} & & 73 \\
\hline & & & .005 & 12 & & & & & & 57 & & & & & & 17. & & & 0.1 & & 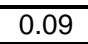 & .044 & & 36.8 & 23.8 & 1. & 564 \\
\hline & & & 007 & & & & & & & & & & & 7 & & & & & & & & .041 & & 31.5 & 23.9 & & 456 \\
\hline & & & .005 & 0.12 & & & 9 & 1.32 & & 1.56 & 0.31 & 58.0 & & & & & 2.86 & & & 1. & & .042 & 1.2 & 28.9 & \begin{tabular}{|l|}
26.5 \\
\end{tabular} & 0.5 & 537 \\
\hline & & & 009 & & & & & & & & & & 10 & & & 17 & 2.67 & & & & & .038 & & 28.8 & 22.5 & 0. & 477 \\
\hline & & & & & & & & & & & & & & & & & & & & & & & & 32.2 & 26.3 & & 401 \\
\hline & & & & & & & & & & & & & & & & & & & & & & $\pi$ & & 29.6 & 8 & & 1050 \\
\hline & & & .005 & & & & & & & 1.66 & & & & & & & & & 0 & & & 040 & & 32.9 & 11.9 & 0. & 519 \\
\hline & & & .005 & & & & 900 & & & & & & & & & & & & & & & & & & 4.1 & & 747 \\
\hline & & & 014 & & & & & & & & & & & & & & & & & & & 40 & & 28.9 & 23.2 & 0. & 1110 \\
\hline & & & 011 & 0.12 & 5.89 & 16.4 & 970 & 1.3 & & 1.69 & 0.4 & & & & & & & & & & & .043 & 1.20 & 29.4 & 26.0 & 0. & 350 \\
\hline & & & & & & & & & & & & & & & & & & & & & & & & & \begin{tabular}{|l|}
25.0 \\
\end{tabular} & & 310 \\
\hline & & & .005 & & & & & & & & & & & & & & & & & & & .045 & & 39.9 & 23.7 & 0. & 582 \\
\hline & & & 0.015 & \begin{tabular}{|l|l|}
0.14 \\
\end{tabular} & 5.82 & 10.0 & 93 & 1.3 & & 1.63 & 0.34 & & & & & 19. & 3.08 & 3.95 & 0.1 & 1. & & .044 & \begin{tabular}{|l}
1.27 \\
\end{tabular} & 26.9 & \begin{tabular}{|l|}
27.1 \\
\end{tabular} & 0.93 & 1025 \\
\hline & & & & & & & & & & & & & & & & & & & & & & & & 77 & \begin{tabular}{|l|}
24.9 \\
\end{tabular} & & 799 \\
\hline & 058 & & .005 & 0.12 & \begin{tabular}{|l|l|}
5.77 \\
\end{tabular} & 8.1 & 900 & & & 1.68 & & & 11.4 & & 2. & 1 & 2.97 & 3.15 & 0 & & 0.10 & .040 & 1.22 & 29.1 & \begin{tabular}{|l|}
23.8 \\
\end{tabular} & \begin{tabular}{|l|}
0.93 \\
\end{tabular} & 560 \\
\hline & & & & 0.09 & & 6 & 850 & & & 1. & 0. & & 10.1 & & & & & & & & & .042 & 1.19 & 30.4 & \begin{tabular}{|l|}
23.2 \\
\end{tabular} & 0.95 & 478 \\
\hline & & & & & & & & & & & & & & & & & & & & & & & & 34.0 & \begin{tabular}{|l|}
20.9 \\
\end{tabular} & & 523 \\
\hline & & 7272372 & 0.005 & 0.09 & 5.80 & 6.8 & 850 & 1.18 & $\overline{0.1}$ & 1.58 & $0.2 \mathrm{~s}$ & 63 & 10.8 & 72 & 2. & $\overline{14}$ & 2.67 & 13.70 & 0.18 & 1.8 & 0.11 & .038 & 1.22 & 31.4 & 23.5 & 0.89 & 580 \\
\hline & & & & & & 8.5 & 970 & & & & & & 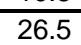 & & & - & & 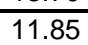 & & & 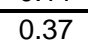 & 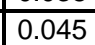 & & 25.2 & 21.4 & \begin{tabular}{|l|}
0.72 \\
\end{tabular} & 1490 \\
\hline
\end{tabular}


Table 1. Location and New Trace Element Geochemical Results for 902 Stream-Sediment Samples collected in the Livengood Area, Livengood Quadrangle, Alaska IS = Insufficient Sample for Analysis

\begin{tabular}{|c|c|c|c|c|c|c|c|c|c|c|c|c|c|c|c|c|c|c|c|c|c|c|c|c|c|c|}
\hline AMPLE & $\begin{array}{l}\text { Mo } \\
\text { ppm }\end{array}$ & \begin{tabular}{l|}
$\mathrm{Na}$ \\
$\%$
\end{tabular} & $\begin{array}{l}\mathrm{Nb} \\
\mathrm{ppm}\end{array}$ & $\begin{array}{c}\mathrm{Ni} \\
\mathrm{ppm}\end{array}$ & $\begin{array}{c}\mathrm{P} \\
\mathrm{ppm}\end{array}$ & $\begin{array}{l}\mathrm{Pb} \\
\mathrm{ppm}\end{array}$ & $\begin{array}{l}\mathrm{Rb} \\
\mathrm{ppm}\end{array}$ & $\begin{array}{c}\mathrm{Re} \\
\mathrm{ppm}\end{array}$ & $\begin{array}{l}\mathrm{S} \\
\%\end{array}$ & $\begin{array}{l}\mathrm{Sb} \\
\mathrm{ppm}\end{array}$ & $\begin{array}{l}\text { Se } \\
\text { ppm }\end{array}$ & $\begin{array}{l}S^{\text {Sn }} \\
\mathrm{ppm}\end{array}$ & \begin{tabular}{c|}
$\mathrm{Sr}$ \\
$\mathrm{ppm}$
\end{tabular} & $\begin{array}{l}\mathrm{Ta}^{*} \\
\mathrm{ppm}\end{array}$ & $\begin{array}{c}\text { Te } \\
\text { ppm }\end{array}$ & $\begin{array}{c}\text { Th } \\
\text { ppm }\end{array}$ & $\begin{array}{l}\mathrm{Ti}^{*} \\
\%\end{array}$ & \begin{tabular}{c|}
$\mathrm{TI}$ \\
$\mathrm{ppm}$
\end{tabular} & $\begin{array}{c}U \\
\mathrm{ppm}\end{array}$ & \begin{tabular}{|c|}
$\mathrm{V}$ \\
$\mathrm{ppm}$
\end{tabular} & $\begin{array}{l}W^{*} \\
\text { ppm }\end{array}$ & $\begin{array}{c}\mathrm{Y} \\
\mathrm{ppm}\end{array}$ & $\begin{array}{l}\mathrm{Zn} \\
\mathrm{ppm}\end{array}$ & $\begin{array}{l}\mathrm{Zr}^{*} \\
\mathrm{ppm}\end{array}$ & $\begin{array}{c}\text { Pulp Wt } \\
\text { grams }\end{array}$ & $\begin{array}{c}\text { Lab } \\
\text { Report }\end{array}$ \\
\hline 1483 & 0.56 & 1.17 & 8.5 & 33.9 & 620 & 36.2 & 60.7 & $<0.002$ & 0.10 & 3.56 & 3 & 1.80 & 223.0 & 0.67 & $<0.05$ & 7.4 & 0.393 & 0.40 & 1.9 & 110 & 1.0 & 16.4 & 184 & 56.6 & 15 & FA04030731 \\
\hline 484 & 0.57 & 14 & 8.3 & 33.7 & 670 & 21.0 & 69.7 & 0.002 & 0.06 & 1.56 & 2 & 2.20 & 202.0 & 0.65 & $<0.05$ & 7.4 & 364 & 0.45 & 1.9 & 117 & 1.0 & 13.0 & 114 & 60.7 & 15 & FA04030731 \\
\hline 85 & 55 & 91 & 7.1 & 33.6 & 950 & 28.2 & 59.2 & $<0.002$ & 0.15 & 2.87 & 3 & 1.90 & 82.0 & 0.54 & 0.05 & 6.6 & .319 & 0.39 & 1.8 & 98 & 0.8 & 14.4 & 205 & 58.2 & & A04030731 \\
\hline 86 & 55 & & 8.4 & 35.8 & 770 & 26.4 & 3.0 & $<0.002$ & 0.10 & 41 & & 2.30 & 16.0 & 0.65 & 0.06 & 7.5 & .395 & & 1.8 & 108 & & 14.2 & 150 & 576 & & \\
\hline 487 & 0.58 & .17 & 8.9 & 32.1 & 700 & 21.3 & 0.3 & $<0.002$ & 0.07 & 2.05 & 2 & 2.00 & 231.0 & 0.67 & $<0.05$ & 7.6 & 0.390 & 0.40 & 2.0 & 106 & 1.2 & 14.6 & 134 & 58.4 & 20 & FA04030731 \\
\hline 88 & 50 & 28 & 8.7 & 33.0 & 690 & 17.1 & 2.3 & $<0.002$ & 0.05 & 1.68 & & 1.90 & 230.0 & 0.67 & 0.06 & 8.5 & 0.400 & 0.38 & 1.9 & 113 & 1.3 & 15.2 & 102 & 65.0 & & FA04030731 \\
\hline 489 & 60 & 19 & 9.2 & 31.6 & 670 & 24.1 & 2.7 & $<0.002$ & 0.08 & 2.02 & 1 & 1.80 & 211.0 & 0.68 & $<0.05$ & 7.1 & 0.397 & 0.50 & 1.6 & 104 & 1.5 & 13.6 & 170 & 57.9 & & FA04030731 \\
\hline$\overline{92}$ & 3.05 & 0.86 & 9.3 & 32.3 & 1090 & 13.8 & 60.0 & $<0.002$ & 0.03 & 4.45 & 1 & 1.80 & 147.0 & 0.68 & 0.08 & 7.7 & 0.396 & 0.44 & 4.1 & 176 & 1.1 & 21.1 & 98 & 74.2 & & FA04030731 \\
\hline & 35 & 88 & 9.3 & 31.1 & 60 & 13.4 & 1.9 & $<0.002$ & 0.04 & 4.16 & & 2.10 & 148.0 & 0.68 & 0.09 & 7.5 & 0.401 & 0.44 & 3.7 & 172 & & 18.4 & 98 & 12.0 & & FA04030731 \\
\hline$\overline{94}$ & 3.02 & 0.59 & 13.0 & 65.1 & 10 & 15.8 & 4.4 & 0.002 & 0.02 & 4.98 & & 2.00 & 136.0 & 0.89 & 0.11 & 5.1 & .855 & 0.32 & 4.1 & 270 & 0.8 & 20.9 & 172 & 82.7 & & FA04030731 \\
\hline 95 & 1.76 & 0.79 & 14.4 & 54.4 & 2100 & 12.6 & 47.3 & $<0.002$ & 0.03 & 3.25 & 2 & 2.20 & 152.0 & \begin{tabular}{|l|}
0.97 \\
\end{tabular} & 0.07 & 6.4 & 0.861 & 0.34 & 3.0 & 194 & 1.0 & 21.3 & 147 & 101.5 & $\overline{19}$ & FA04030731 \\
\hline & 4 & & 7.9 & 41.8 & 300 & 11.3 & 51.8 & $<0.002$ & 0.08 & 2.08 & & 1.90 & 154.5 & 0.57 & 0.05 & 6.1 & 0.365 & 0.37 & 3.1 & 126 & & 26.1 & 119 & 60.1 & & \\
\hline & 1.87 & 0.87 & 11.0 & 49.3 & 1600 & 12.7 & 53.6 & $<0.002$ & 0.03 & 2.83 & 1 & 2.50 & 160.5 & \begin{tabular}{|l|l|}
0.74 \\
\end{tabular} & 0.07 & 7.0 & 0.574 & 0.35 & 2.9 & 174 & 1.0 & 21.0 & 154 & 84.3 & & $\overline{\text { FA0403 }}$ \\
\hline 15 & 1.49 & 0.90 & 10.0 & 47.6 & 1520 & 11.3 & 49.1 & $<0.002$ & 0.03 & 2.70 & 1 & 1.70 & 164.5 & 0.71 & 0.07 & 5.8 & 0.479 & 0.35 & 2.9 & 164 & 1.0 & 19.0 & 122 & 70.4 & 13 & FA04030731 \\
\hline & 08 & 82 & 10.5 & 60.8 & 2400 & 15.3 & 59.1 & $<0.002$ & 0.04 & 3.75 & & 1.90 & 166.5 & \begin{tabular}{|l|l|}
0.74 \\
\end{tabular} & 0.09 & 7.0 & 0.484 & 0.44 & 3.6 & 217 & & 23.0 & 207 & 83.5 & & FA0403 \\
\hline & 0.60 & 1.16 & 11.2 & 105.0 & 750 & 8.7 & 46.9 & $<0.002$ & 0.01 & 1.02 & 1 & 1.50 & 190.0 & 0.82 & $<0.05$ & 10.0 & 0.502 & 0.28 & 2.2 & 122 & 1.0 & 17.1 & 79 & 84.3 & 3 & FA0403 \\
\hline & 31 & 1.14 & 9.1 & 128.0 & 730 & 9.6 & 51.1 & $<0.002$ & 0.02 & 1.00 & & 1.30 & 182.0 & 0.68 & $<0.05$ & 7.6 & 0.415 & 0.29 & 1.9 & 115 & 1. & 15.6 & & 72.7 & & FA040 \\
\hline & 0.63 & 1.04 & 8.7 & 116.5 & 720 & 8.2 & 51.0 & $<0.002$ & 0.02 & 1.38 & 1 & 1.20 & 158.0 & 0.60 & $<0.05$ & 6.4 & & 0.30 & 2.4 & 122 & & 14.0 & & & & FA040 \\
\hline & 0.70 & 1.04 & 8.7 & 111.5 & 700 & 9.4 & 55.8 & $<0.002$ & 0.02 & 1.18 & 1 & 1.30 & 158.5 & 0.61 & $<0.05$ & 6.6 & 0.390 & 0.34 & 1.7 & 122 & 1.0 & 14.3 & 97 & 64.6 & 2 & FA04030731 \\
\hline & 68 & 1.02 & 6.8 & 266.0 & 620 & 7.8 & 43.3 & $<0.002$ & 0.05 & 0.88 & & 1.70 & 132.5 & \begin{tabular}{|l|}
0.49 \\
\end{tabular} & $<0.05$ & 5.5 & 0.389 & 0.26 & 1.4 & 121 & 0.8 & 15.8 & 7 & 66.4 & & FA0403 \\
\hline & & 07 & 7.6 & 226.0 & 590 & 8.8 & 44.5 & $<0.002$ & 0.03 & 1.22 & & 1.40 & 150.0 & 0.54 & $<0.05$ & 5.4 & 0.410 & 0.30 & 1.5 & 130 & 1. & 14.6 & $\overline{8 \varepsilon}$ & 65 & & $\mathrm{FAO}$ \\
\hline & 1.06 & 1.19 & 10.0 & 47.7 & 760 & 11.4 & 57.5 & $<0.002$ & 0.02 & 1.28 & & 1.60 & 196.0 & 0.74 & 0.05 & 8.1 & 0.420 & 0.37 & 2.1 & 118 & 0.9 & 16.7 & 81 & 73.7 & 3 & FA04030731 \\
\hline & 87 & 1.03 & 11.4 & 84.3 & 630 & 11.7 & 78.1 & $<0.002$ & 0.06 & 1.99 & & 2.10 & 138.5 & 0.74 & $<0.05$ & 7.2 & .370 & 0.55 & 2.2 & 129 & 1.7 & 18.0 & 12 & 91.2 & & FA040 \\
\hline & 86 & .95 & 10.6 & 98.7 & 600 & 12.1 & 79.5 & $<0.002$ & 0.07 & 2.37 & 2 & 2.10 & 138.0 & 0.71 & 0.06 & 7.2 & 0.324 & 0.62 & 2.3 & 130 & 1.6 & 19.6 & 124 & 92.3 & & FA040 \\
\hline & 1.01 & 1.23 & 10.8 & 136.5 & 810 & 8.5 & 49.6 & $<0.002$ & 0.03 & 1.33 & & 1.50 & 179.0 & 0.76 & 0.05 & 7.9 & 5.516 & 0.33 & 2.0 & 130 & 1.2 & 17.9 & 85 & 86.5 & & FA040 \\
\hline & 0.78 & 1.39 & 10.0 & 29.4 & 910 & 11.3 & 55.4 & $<0.002$ & 0.03 & 1.06 & & 1.50 & 247.0 & 0.69 & $<0.05$ & 8.1 & 0.392 & 0.33 & 2.2 & 107 & 1.0 & 17.6 & & $74 . \varepsilon$ & & FA0403 \\
\hline & 0.79 & 1.30 & 8.3 & 29.1 & 750 & 12.2 & 62.9 & $<0.002$ & 0.03 & 1.00 & & 1.70 & 222.0 & 0.59 & $<0.05$ & 7.6 & 0.345 & 0.40 & 1.9 & 112 & $\overline{0.8}$ & 15.7 & 85 & 62.2 & & FA040 \\
\hline 23 & 0.68 & 1.29 & 9.3 & 25.7 & 710 & 11.5 & 53.8 & $<0.002$ & 0.03 & 0.97 & & 1.50 & 219.0 & \begin{tabular}{|l|}
0.67 \\
\end{tabular} & $<0.05$ & 7.5 & 0.395 & 0.33 & 2.0 & 108 & 0.9 & 15.6 & 96 & 67.1 & & FA04030732 \\
\hline & & 1.33 & 10.5 & 28.1 & 820 & 11.1 & 62.1 & $<0.002$ & 0.03 & 0.96 & & & 225.0 & \begin{tabular}{|l|l|}
0.77 \\
\end{tabular} & $<0.05$ & 8.7 & & 0.39 & 2.2 & 115 & & 16.8 & 85 & 74 & & \\
\hline & 0.88 & 1.30 & 9.9 & 30.9 & 790 & 11.9 & 63.8 & $<0.002$ & 0.04 & 1.00 & & 1.60 & 217.0 & 0.71 & $<0.05$ & 8.0 & .404 & 0.40 & 2.1 & 114 & 1.2 & 17.0 & 157 & 70.2 & & FA040 \\
\hline 1526 & 0.72 & \begin{tabular}{|l|l|}
1.34 \\
\end{tabular} & 10.4 & 27.5 & 760 & 10.2 & 52.0 & $<0.002$ & 0.02 & 0.88 & & 1.50 & 225.0 & \begin{tabular}{|l|}
0.79 \\
\end{tabular} & $<0.05$ & 8.1 & 447 & 0.30 & 2.2 & 1 & 1.2 & 16.8 & $11 !$ & 75.7 & & FA04030732 \\
\hline & & 1.25 & 9.9 & 30.1 & 840 & 11.7 & 58.1 & .002 & 0.03 & 1.06 & & 1.60 & 212.0 & 0.74 & $<0.05$ & 8.1 & & 0.35 & 2.1 & 115 & & 16.6 & 142 & 72.6 & & \\
\hline 29 & 1.07 & 1.15 & 9.2 & 35.6 & 900 & 11.6 & 55.3 & $<0.002$ & 0.05 & 1.03 & & 1.70 & 194.5 & 0.68 & 0.05 & 7.2 & 0.399 & 0.34 & 2.1 & 116 & 0.9 & 18.6 & 142 & 68.6 & & FA04030732 \\
\hline & 1.66 & 1.21 & 9.6 & 32.0 & 940 & 12.5 & 62.6 & $<0.002$ & 0.05 & 1.10 & & 1.70 & 214.0 & 0.70 & 0.06 & 7.8 & 0.387 & 0.35 & 2.0 & 118 & 1.2 & 17.5 & 140 & 70.7 & & FA04030732 \\
\hline & & & 10.2 & 28.7 & 820 & 11.8 & 60.9 & $<0.002$ & 0.03 & 0.98 & & & 222.0 & 0.73 & & 8.4 & & & 2.1 & & & & & 72.9 & & FA04030732 \\
\hline 32 & 0.73 & 1.40 & 10.6 & 27.3 & 840 & 11.0 & 58.8 & $<0.002$ & 0.03 & 0.92 & $\perp$ & 1.60 & 237.0 & 0.75 & $<0.05$ & 9.9 & 0.463 & 0.34 & 2.3 & 118 & 1.3 & 18.4 & 106 & 79.8 & & FA04030732 \\
\hline 1533 & 1.01 & 1.23 & 9.5 & 30.8 & 770 & 11.7 & 63.8 & $<0.002$ & 0.04 & 1.10 & 1 & 1.60 & 209.0 & 0.68 & 0.05 & 7.3 & 0.372 & 0.39 & 1.9 & 112 & 1.1 & 15.5 & 112 & 65.6 & & FA04030732 \\
\hline & & & 9.3 & & 870 & & & & 0.04 & & & & & 0.69 & $<0.05$ & 8.4 & & & & & & & & 68.8 & & FA04030732 \\
\hline 1535 & 0.80 & 1.34 & 9.7 & 27.3 & 820 & 11.1 & 56.7 & $<0.002$ & 0.04 & 1.00 & 1 & 1.50 & 224.0 & 0.69 & $<0.05$ & 7.5 & 0.401 & 0.34 & 2.0 & 111 & 1.1 & 15.8 & 109 & 68.4 & & FA04030732 \\
\hline & 0.76 & 1.39 & 9.9 & 25.5 & 750 & 10.9 & 55.7 & $<0.002$ & 0.02 & 0.96 & & 1.50 & 237.0 & 0.72 & $<0.05$ & 7.7 & 0.411 & 0.32 & 2.0 & 109 & 1. & 15.8 & 119 & 67.7 & & FA04030732 \\
\hline & 0.65 & 1.34 & 10.4 & 24.3 & 760 & 10.5 & 51.2 & & 0.02 & 0.92 & & 1.50 & 225.0 & 0.76 & & 8.9 & & & 2.2 & 106 & 1.3 & & 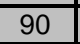 & 77.0 & & FA04030732 \\
\hline 1538 & 0.69 & 1.35 & 8.6 & 26.3 & 750 & 10.8 & 59.7 & $<0.002$ & 0.03 & 0.93 & 1 & 1.60 & 224.0 & 0.63 & $<0.05$ & 8.0 & 0.358 & 0.35 & 1.9 & 106 & 1.0 & 15.6 & 83 & 62.3 & 20 & FA04030732 \\
\hline 1542 & 1.87 & 0.79 & 8.0 & 30.5 & 1100 & 11.4 & 50.6 & $<0.002$ & 0.07 & 8.50 & & 1.70 & 138.5 & 0.59 & 0.07 & 6.7 & 0.373 & 0.36 & 2.3 & 136 & 3.2 & 17.8 & 141 & 61.2 & 14 & FA04030732 \\
\hline
\end{tabular}


Table 1. Location and New Trace Element Geochemical Results for 902 Stream-Sediment Samples collected in the Livengood Area, Livengood Quadrangle, Alaska IS = Insufficient Sample for Analysis

\begin{tabular}{|c|c|c|c|c|c|c|c|c|c|c|c|c|c|c|c|c|c|c|c|c|c|c|c|c|c|c|c|}
\hline MPLE & $\mathrm{ME}$ & $\mathrm{M} \mathrm{N}$ & $\begin{array}{l}\mathrm{Au} \\
\mathrm{ppm}\end{array}$ & $\begin{array}{c}\mathrm{Ag} \\
\mathrm{ppm}\end{array}$ & $\begin{array}{l}\text { Al } \\
\%\end{array}$ & $\begin{array}{l}\text { As } \\
\text { ppm }\end{array}$ & $\begin{array}{l}\mathrm{Ba}^{*} \\
\mathrm{ppm}\end{array}$ & $\begin{array}{c}\mathrm{Be} \\
\mathrm{ppm}\end{array}$ & $\begin{array}{c}\mathrm{Bi} \\
\mathrm{ppm}\end{array}$ & $\begin{array}{c}\mathrm{Ca} \\
\%\end{array}$ & $\begin{array}{c}\mathrm{Cd} \\
\mathrm{ppm}\end{array}$ & $\begin{array}{c}\text { Ce } \\
\text { ppm }\end{array}$ & $\begin{array}{l}\text { Co } \\
\text { ppm }\end{array}$ & $\begin{array}{l}\mathrm{Cr} \\
\mathrm{ppm}\end{array}$ & $\begin{array}{c}\text { Cs } \\
\mathrm{ppm}\end{array}$ & $\begin{array}{c}\mathrm{Cu} \\
\mathrm{ppm}\end{array}$ & $\begin{array}{l}\mathrm{Fe} \\
\% \\
\end{array}$ & $\begin{array}{c}\mathrm{Ga} \\
\mathrm{ppm}\end{array}$ & $\begin{array}{c}\mathrm{Ge} \\
\mathrm{ppm}\end{array}$ & $\begin{array}{c}\mathrm{Hf} \\
\mathrm{ppm}\end{array}$ & $\begin{array}{l}\mathrm{Hg} \\
\mathrm{ppm}\end{array}$ & $\begin{array}{c}\text { In } \\
\mathrm{ppm}\end{array}$ & $\begin{array}{l}\mathrm{K} \\
\% \\
\end{array}$ & $\begin{array}{c}\mathrm{La} \\
\mathrm{ppm}\end{array}$ & \begin{tabular}{c|}
$\mathrm{Li}$ \\
$\mathrm{ppm}$
\end{tabular} & $\begin{array}{c}\mathrm{Mg} \\
\% \\
\end{array}$ & $\begin{array}{l}\mathrm{Mn} \\
\mathrm{ppm}\end{array}$ \\
\hline 543 & & & 0.006 & & & 57.1 & 1340 & \begin{tabular}{|l|}
1.08 \\
\end{tabular} & 0.12 & 2.19 & 0.85 & 47.5 & 39.8 & 59 & 1.74 & 20.1 & 8.48 & & 0.33 & 1.2 & & 0.032 & 0.73 & 23.1 & 14.3 & \begin{tabular}{|l|}
0.65 \\
\end{tabular} & 5290 \\
\hline & & & & 11 & & 7.5 & & \begin{tabular}{|l|l|}
1.14 \\
\end{tabular} & & & 0.43 & 2.1 & & & & 5.2 & & 11.60 & & 1.5 & 20 & & 0.94 & 25.8 & \begin{tabular}{|l|}
19.2 \\
\end{tabular} & 1.30 & 540 \\
\hline & & & 008 & 16 & & 2.3 & 960 & \begin{tabular}{|l|l|}
1.12 \\
\end{tabular} & & & 0.39 & 1.7 & 1.5 & & .87 & 252 & 94 & 3.05 & 0.20 & & 27 & 041 & 1.05 & 25.0 & 0.6 & & 035 \\
\hline & & & & & & & & 1.57 & & & & & & & & & & & & & & & & & & & 91 \\
\hline & & & .005 & 12 & 3 & 0.5 & 820 & 1.18 & 13 & 73 & 0.41 & 3.3 & 5.1 & 77 & .16 & 6.8 & .26 & 2.90 & 0.19 & & 15 & 040 & 1.09 & 30.5 & 0.7 & 0.93 & 025 \\
\hline & & & 008 & 15 & 32 & 10.8 & 960 & 1.10 & 12 & 70 & 1.38 & 56.4 & 57 & 65 & 2.56 & 20.3 & 3.29 & 1.45 & 0.19 & & 36 & 037 & 0.97 & 25.4 & 8.8 & 0.79 & 190 \\
\hline & & & & 0.12 & & 7.1 & 860 & 1.22 & & & 0.24 & & & & & & & & & & & & 1.12 & & & & 443 \\
\hline & & & .005 & 10 & 75 & 6.4 & 870 & 1.18 & 11 & 64 & 0.21 & 59.6 & 9.5 & 69 & 2.15 & 13.2 & 2.59 & 3.10 & 0.16 & & 14 & 040 & 1.19 & 29.0 & 1.8 & 0.93 & 81 \\
\hline & & & 17 & 10 & & & 30 & 1.10 & 12 & 81 & 0.34 & ..5 & 0.3 & 14 & 2.50 & 5.9 & 3.22 & 3.90 & 0.19 & & 17 & 043 & 0.98 & 29.7 & 4.6 & 1.30 & 726 \\
\hline & & & 05 & 10 & & 6.5 & 50 & 1.00 & 13 & 70 & 0.37 & 5.7 & 5.0 & & 2.50 & 26.6 & 3.38 & 3.45 & 0.20 & & 22 & (045 & 0.97 & 27.1 & & & 852 \\
\hline & & & 0.008 & 0.07 & 54 & 7.6 & 500 & 1.12 & 12 & 26 & 0.17 & 56.8 & 3 & & 2.27 & 21.4 & 3.58 & 14.05 & 0.19 & & 19 & $\begin{array}{l}.045 \\
\end{array}$ & 0.94 & 27.1 & 55.7 & 1.19 & 60 \\
\hline & & & .005 & 0.12 & & 6. & & 1.10 & 14 & 60 & 0.66 & & & & 08 & 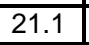 & . & 14.35 & 0.17 & & 0 & 045 & 1.06 & 24.5 & 26.3 & & 570 \\
\hline & & & .012 & 16 & & 16.5 & 90 & 1.18 & 15 & 44 & 1.02 & 60. & 7 & & $\overline{9 s}$ & & .11 & 3.30 & 0.24 & & 7 & 046 & & 7.0 & 5.7 & & 4450 \\
\hline & & & .005 & 0.11 & 15 & 7.8 & 610 & 0.89 & 0.09 & 26 & 0.31 & 44.8 & 9.4 & 72 & 1.64 & 14.4 & 2.34 & 9.55 & 0.15 & 1. & .16 & \begin{tabular}{|l|l|}
.033 \\
\end{tabular} & 0.74 & 22.3 & 7.2 & 0.93 & 487 \\
\hline & & & & 0.31 & & & & 2.39 & & & & & & & & & 3.50 & 24.70 & 0.24 & & & 062 & & $\overline{2}$ & 2.5 & & 242 \\
\hline & & & 0.009 & \begin{tabular}{l|l}
0.18 \\
\end{tabular} & & 6.0 & 90 & 0.95 & & 66 & 0.26 & 33.0 & & & $\overline{01}$ & 25.5 & 11.95 & 9.06 & 0.3 & & 76 & 030 & & 6.5 & 10.5 & & 68 \\
\hline & & & .021 & \begin{tabular}{l|l}
0.28 \\
\end{tabular} & 77 & 5.5 & 0 & 0.97 & 15 & 0 & 0.40 & & 9.6 & & & 8.9 & .05 & $\begin{array}{l}7.6 \\
\end{array}$ & 0.41 & & 78 & 34 & & 6.4 & 9.2 & & 500 \\
\hline & & & & 0.24 & & 8 & 0 & \begin{tabular}{ll|}
1.44 \\
\end{tabular} & & & & & & & & & & 85 & & & & & & & 7 & & \\
\hline & & & & 0.11 & 97 & 36.1 & 980 & 1.17 & 14 & 04 & 0.19 & & 8. & & 2.77 & .1 & 4.13 & 2.70 & 23 & 1. & 52 & & 1. & 27.4 & \begin{tabular}{l|l}
8.3 \\
\end{tabular} & 71 & 12 \\
\hline & & & & \begin{tabular}{l|l|}
0.35 \\
\end{tabular} & & & 50 & 1.44 & & & & & & & & & & 5.4 & 0.20 & & & & & 30.9 & y & & 86 \\
\hline & & & 08 & 0.09 & & 26.6 & 0 & \begin{tabular}{|l|l|}
1.28 \\
\end{tabular} & & 33 & 0.23 & & & & 265 & & & & & & & & & 29.9 & & & 17 \\
\hline & & & .005 & $\begin{array}{ll}0.14 \\
\end{array}$ & 57 & 11.2 & 900 & 1.26 & 0.14 & .42 & $\begin{array}{ll}0.27 \\
\end{array}$ & 67.0 & & & 35 & 20. & 3.18 & 13.70 & 0.23 & & .32 & $\begin{array}{l}.046 \\
\end{array}$ & 1.1 & 32.3 & 1.6 & .90 & 449 \\
\hline & & & 007 & 14 & & 22.2 & & \begin{tabular}{|l|l|}
1.17 \\
\end{tabular} & & & 0.19 & & & & 6 & & & 4.10 & 23 & & & $\begin{array}{l}.044 \\
\end{array}$ & & 30.4 & 2 & & 447 \\
\hline & & & & 13 & & 5.6 & 10 & 1.20 & & & & & & & & & & & & & & & & 32.4 & & & 549 \\
\hline & & & .00 & 0.19 & & 13. & 1190 & 1.44 & & & 174 & & & & $4<$ & 25 & & 3.50 & 0.25 & & & \begin{tabular}{|l|}
.048 \\
\end{tabular} & $\overline{0 . \varepsilon}$ & 28.4 & 21.0 & 0.6 & 10000 \\
\hline & & & & & & & & & & & & & & & & & & 3. & & & & 5 & & 3.9 & 6 & & 88 \\
\hline & & & 005 & 11 & & & 0 & 1.16 & & & 0.22 & & & & & & & 40 & & & & & & 30.4 & & & 25 \\
\hline & & & $\overline{0 \pi}$ & & & & & & & & & & & & & & & -1 & 18 & & & & & 7.1 & 1.8 & & 87 \\
\hline & & & & & & & & & & & & & & & & & & & & & & & & & & & 43 \\
\hline & & & & & & & & & & & & & & & & & & & & & & & & & & & $\overline{03}$ \\
\hline & & & .005 & 13 & & & & 1.32 & & & & & & & & & & 0 & 20 & & & 46 & & 3.8 & 2.7 & & 30 \\
\hline & & & & & & & 900 & 1.25 & & & & & & & & & & & & & & & & & 2.2 & & 16 \\
\hline & & & $\overline{00}$ & & & & & 1. & & & & & & & & & & & & & & & & 1.2 & 20. & & 43 \\
\hline & & & .005 & 18 & & & 730 & 1.06 & & 0.98 & 0.18 & & & & & & & 6.00 & $\overline{0}$ & & 21 & $\begin{array}{l}.049 \\
\end{array}$ & & .4 & 29.1 & & 402 \\
\hline & & & & & & & & & & & & & & & & & & & & & & & & & 4.4 & & 35 \\
\hline & & & 00 & & & & & & & & & & & & & & & & & & & 9 & & & 0.0 & & 19 \\
\hline & & & .005 & $\begin{array}{ll}0.12 \\
\end{array}$ & & & 69 & 1.04 & & & 0.17 & & & & & & & 14.85 & 0.18 & & & 046 & \begin{tabular}{|l|l}
1.0 \\
\end{tabular} & 33.3 & 26.4 & 0.81 & 56 \\
\hline & & & & & & & & & & & & & & & & & & & & & & & & & & & 50 \\
\hline & & & .005 & & & 14 & & 1.0 & & & & & & & & & & & 0.22 & & & 051 & & 30.7 & 2.9 & & 380 \\
\hline & & & $<0.005$ & \begin{tabular}{|l|l|} 
\\
\end{tabular} & 5.7 & 7. & 650 & 0.98 & & & 0.16 & & 9.3 & & & 12.9 & & 14.20 & 0.20 & 2.1 & & 046 & & & 24.7 & & 94 \\
\hline & & & & & & & & & & & & & & & & & & & & & & & & & & & 32 \\
\hline & & & 0.013 & $\begin{array}{ll}0.09 \\
\end{array}$ & 5.81 & 9.2 & 660 & 0.92 & 0.1 & 1.3 & 0.16 & 81. & 12.2 & 107 & 2.4 & 11.6 & 3.0 & 14 & 0.21 & 2.0 & & 046 & 1.0 & 38.8 & 24.8 & 81 & 554 \\
\hline & & & 0.026 & & & 15.1 & 720 & & & & & & & & & 17.6 & & & & & & & & 0.1. & \begin{tabular}{l|l}
36.5 \\
\end{tabular} & & 454 \\
\hline
\end{tabular}


Table 1. Location and New Trace Element Geochemical Results for 902 Stream-Sediment Samples collected in the Livengood Area, Livengood Quadrangle, Alaska IS = Insufficient Sample for Analysis

\begin{tabular}{|c|c|c|c|c|c|c|c|c|c|c|c|c|c|c|c|c|c|c|c|c|c|c|c|c|c|c|}
\hline AMPLE & $\begin{array}{l}\text { Mo } \\
\text { ppm }\end{array}$ & \begin{tabular}{l|}
$\mathrm{Na}$ \\
$\%$
\end{tabular} & $\begin{array}{l}\mathrm{Nb} \\
\mathrm{ppm}\end{array}$ & $\begin{array}{c}\mathrm{Ni} \\
\mathrm{ppm}\end{array}$ & $\begin{array}{c}\mathrm{P} \\
\mathrm{ppm}\end{array}$ & $\begin{array}{l}\mathrm{Pb} \\
\mathrm{ppm}\end{array}$ & $\begin{array}{l}\mathrm{Rb} \\
\mathrm{ppm}\end{array}$ & $\begin{array}{c}\mathrm{Re} \\
\mathrm{ppm}\end{array}$ & $\begin{array}{l}\mathrm{S} \\
\%\end{array}$ & $\begin{array}{c}\text { Sb } \\
\mathrm{ppm}\end{array}$ & $\begin{array}{c}\text { Se } \\
\text { ppm }\end{array}$ & $\begin{array}{l}S^{S n^{*}} \\
\text { ppm }\end{array}$ & \begin{tabular}{c|}
$\mathrm{Sr}$ \\
$\mathrm{ppm}$
\end{tabular} & $\begin{array}{l}\mathrm{Ta}^{*} \\
\mathrm{ppm}\end{array}$ & $\begin{array}{c}\text { Te } \\
\text { ppm }\end{array}$ & $\begin{array}{c}\text { Th } \\
\mathrm{ppm}\end{array}$ & $\begin{array}{l}\mathrm{Ti}^{*} \\
\%\end{array}$ & \begin{tabular}{c|}
$\mathrm{TI}$ \\
$\mathrm{ppm}$
\end{tabular} & $\begin{array}{c}U \\
\mathrm{ppm}\end{array}$ & \begin{tabular}{|c|}
$\mathrm{V}$ \\
$\mathrm{ppm}$
\end{tabular} & $\begin{array}{l}W^{*} \\
\text { ppm }\end{array}$ & $\begin{array}{c}\mathrm{Y} \\
\mathrm{ppm}\end{array}$ & $\begin{array}{l}\mathrm{Zn} \\
\mathrm{ppm}\end{array}$ & $\begin{array}{l}\mathrm{Zr}^{*} \\
\mathrm{ppm}\end{array}$ & $\begin{array}{c}\text { Pulp Wt } \\
\text { grams }\end{array}$ & $\begin{array}{c}\text { Lab } \\
\text { Report }\end{array}$ \\
\hline 1543 & 1.41 & 0.61 & 5.9 & 31.2 & 1620 & 9.7 & 40.9 & $<0.002$ & 0.08 & 2.30 & 1 & 1.80 & 141.5 & 0.41 & 0.11 & 5.9 & 0.259 & 0.28 & 1.9 & \begin{tabular}{|l|l|}
114 \\
\end{tabular} & 0.7 & 17.2 & 84 & 46.5 & 11 & FA04030732 \\
\hline 544 & 0.76 & 92 & 7.7 & 27.9 & 1320 & 9.8 & 50.1 & 0.002 & 0.06 & 1.48 & 1 & 1.40 & \begin{tabular}{l|l|}
71.5 \\
\end{tabular} & 0.55 & 0.08 & 6.6 & 0.318 & 0.33 & 1.7 & 104 & 0.8 & 15.1 & 77 & 54.9 & 11 & FA04030732 \\
\hline 45 & 1.14 & 94 & 8.1 & 32.6 & 1060 & 11.6 & 49.1 & $<0.002$ & 0.05 & 2.27 & 1 & 1.50 & 60.0 & 0.60 & 0.08 & 6.8 & 0.423 & 0.33 & 2.0 & 135 & 1.1 & 14.7 & 105 & 59.4 & & A04030732 \\
\hline 46 & 1.05 & 13 & 10.4 & 36.7 & 720 & 14.7 & 66.5 & $<0.002$ & 0.03 & 2.09 & & 2.00 & 84.5 & 0.75 & 0.06 & 9.2 & 0.457 & 0.45 & 2.3 & 140 & 1.2 & 17.4 & & 76.0 & & \\
\hline 549 & 1.10 & 1.27 & 9.4 & 27.8 & 850 & 10.8 & 52.6 & 0.002 & 0.04 & 1.18 & 1 & 1.50 & 221.0 & 0.72 & $<0.05$ & 8.0 & 0.394 & 0.32 & 2.1 & 110 & 1.0 & 16.8 & 120 & 66.9 & 13 & A04030732 \\
\hline 50 & 1.34 & .00 & 7.8 & 31.9 & 1140 & 10.2 & 51.4 & $<0.002$ & 0.09 & 1.13 & 1 & 1.60 & 184.0 & 0.57 & 0.05 & 6.6 & 0.324 & 0.32 & 1.8 & 95 & 0.7 & 14.7 & 121 & 53.0 & 1 & FA04030732 \\
\hline 551 & 0.68 & 1.33 & 10.8 & 26.6 & 770 & 10.6 & 51.0 & $<0.002$ & 0.02 & 1.08 & 1 & 1.50 & 226.0 & 0.81 & $<0.05$ & 9.1 & 0.455 & 0.29 & 2.3 & 112 & 1.1 & 17.5 & 74 & 79.0 & & FA04030732 \\
\hline$\overline{52}$ & 0.64 & 1.42 & 9.8 & 24.4 & 730 & 10.3 & 53.1 & $<0.002$ & 0.02 & 1.00 & 1 & 1.50 & 229.0 & 0.71 & $<0.05$ & 7.7 & 0.405 & 0.32 & 2.0 & 108 & 1.1 & 15.1 & 69 & 64.0 & & FA04030732 \\
\hline & & 29 & 8.0 & 65.7 & 670 & 10.3 & 52.1 & $<0.002$ & 0.05 & 0.94 & & 1.60 & 166.5 & 0.59 & $<0.05$ & 7.3 & 0.426 & 0.32 & 1.6 & 118 & & 18.4 & & 61.3 & & FA04030732 \\
\hline 1560 & 0.68 & 1.23 & 7.3 & 56.5 & 710 & 10.1 & 50.3 & $<0.002$ & 0.06 & 0.85 & 1 & .60 & 152.5 & \begin{tabular}{|c|}
0.54 \\
\end{tabular} & $<0.05$ & 6.6 & 0.412 & 0.28 & 1.6 & 120 & 1.1 & 19.0 & 88 & 63.2 & & FA040 \\
\hline 1561 & 0.70 & 1.54 & 7.7 & 43.0 & 590 & 10.0 & 49.7 & $<0.002$ & 0.03 & 0.84 & 1 & 1.40 & 143.0 & 0.59 & 0.05 & 7.2 & 0.448 & 0.25 & 1.7 & 118 & 0.9 & 15.6 & 71 & 65.3 & 2 & FA04030732 \\
\hline & 0.89 & .08 & 7.0 & 44.7 & 700 & 10.7 & 60.6 & $<0.002$ & 0.06 & 1.04 & & 1.70 & 162.0 & 0.51 & $<0.05$ & 6.3 & 0.345 & 0.37 & 1.6 & 112 & & 15.7 & & & & \\
\hline 1563 & 1.46 & 0.82 & 7.0 & 55.3 & 790 & 10.5 & 57.2 & $<0.002$ & 0.07 & 1.18 & 1 & 1.60 & 145.5 & 0.51 & 0.07 & 6.9 & 0.323 & 0.42 & 1.7 & 124 & 0.9 & 18.2 & 147 & 59.0 & & FA04030732 \\
\hline 1564 & 0.43 & 0.84 & 7.7 & 25.1 & 1740 & 9.2 & 44.6 & $<0.002$ & 0.08 & 1.44 & 1 & 1.20 & 143.0 & 0.55 & $<0.05$ & 5.7 & 0.367 & 0.24 & 1.8 & 92 & 0.7 & 13.4 & 88 & 54.8 & 1 & FA04030732 \\
\hline 67 & 08 & 0.33 & 6.0 & 20.7 & 4840 & 26.9 & 105.5 & 0.002 & 0.06 & 5.31 & & 2.80 & 260.0 & 0.46 & 0.21 & 9.3 & 0.250 & 1.26 & 24.6 & 753 & & 66.8 & & 125.5 & & FA040 \\
\hline & 12.60 & 0.33 & 3.8 & 24.5 & 6480 & 9.6 & 37.9 & $<0.002$ & 0.08 & 2.98 & 2 & 1.30 & 116.5 & 0.29 & 0.19 & 4.3 & 0.168 & 0.29 & 6.9 & 273 & 0.7 & 27.9 & $\overline{5 \varepsilon}$ & 50.9 & & FA040 \\
\hline & 9.05 & 0.35 & 3.5 & 28.1 & 10 & 9.0 & 32.0 & $<0.002$ & 0.10 & 2.58 & & 1.20 & 110.5 & 0.25 & 0.20 & 4.0 & 0.144 & 0.29 & 6.1 & 239 & 0.6 & 26.6 & & 40.3 & & FA040 \\
\hline & 4.06 & 0.74 & 8.7 & 25.0 & 60 & 20.4 & 79.6 & $<0.002$ & 0.06 & 1.88 & 2 & 2.30 & 140.0 & 0.63 & 0.14 & 8.2 & & 0.58 & 4.6 & & & 19.5 & & & & EAOAn \\
\hline 15 & 1.95 & 0.95 & 8.1 & 22.8 & 2370 & 12.6 & 53.7 & $<0.002$ & 0.04 & 1.50 & 1 & 1.60 & 165.0 & 0.60 & 0.07 & 7.4 & 0.340 & 0.35 & 3.3 & 144 & 1.0 & 17.0 & 59 & 61.1 & 2 & FA04030732 \\
\hline 74 & 9.97 & 0.68 & 7.7 & 17.9 & 3090 & 22.7 & 65.2 & 0.002 & 0.08 & 8.55 & & 2.00 & 185.0 & 0.59 & 0.15 & 8.8 & 0.307 & 0.53 & 10.3 & 444 & 1.4 & 34.4 & & 79.8 & & FA0403 \\
\hline & 1.84 & 19 & 9.2 & 28.4 & 1070 & 12.5 & 61.5 & $<0.002$ & 0.03 & 1.36 & & 1.70 & 194.5 & \begin{tabular}{|l|l|}
0.68 \\
\end{tabular} & 0.06 & 8.3 & 0.390 & 0.37 & 2.5 & 126 & 1. & 16.8 & & 69 & & FA04 \\
\hline 76 & 1.06 & 1.23 & 9.7 & 27.2 & 920 & 13.2 & 56.2 & $<0.002$ & 0.03 & 1.34 & & 1.70 & 201.0 & 0.73 & $<0.05$ & 8.7 & 0.419 & 0.35 & 2.6 & 122 & 1.1 & 17.2 & & 65.0 & & FA04030732 \\
\hline 77 & 1.89 & 1.16 & 9.7 & 26.0 & 1010 & 13.0 & 58.9 & $<0.002$ & 0.04 & 1.37 & & 1.80 & 188.0 & 0.70 & 0.05 & 8.1 & 399 & 0.37 & 2.5 & 131 & 1.2 & 16.0 & & 65.6 & & FA040 \\
\hline & 0.69 & 1.20 & 9.4 & 26.8 & 680 & 11.7 & 52.1 & $<0.002$ & 0.03 & 1.18 & 1 & 1.70 & 187.0 & 0.75 & $<0.05$ & 8.7 & 0.407 & 0.36 & 2.5 & 110 & 1.0 & 17.3 & 82 & 65.1 & & FA04C \\
\hline 79 & 2.62 & 0.79 & 6.9 & 36.1 & 1040 & 12.6 & 51.7 & $<0.002$ & 0.07 & 1.54 & & 1.50 & 144.5 & 0.52 & 0.08 & 7.3 & 0.287 & 0.38 & 2.8 & 126 & 0.9 & 22.4 & 147 & 51.8 & & FA04 \\
\hline 30 & 0.83 & \begin{tabular}{|l|l|}
1.31 \\
\end{tabular} & 10.0 & 30.4 & 840 & 12.2 & 57.1 & $<0.002$ & 0.02 & 1.24 & & 1.80 & 211.0 & 0.86 & 0.1 & 9.6 & 437 & 0.34 & 2.4 & 120 & 0.9 & 17.0 & & 64.5 & & FA0403 \\
\hline & 0.64 & 1.31 & 9.7 & 26.6 & 710 & 10.9 & 53.7 & $<0.002$ & 0.02 & 1.06 & & 1.60 & 207.0 & 0.72 & $<0.05$ & 8.2 & 425 & 0.32 & 2.2 & 110 & 1.3 & 15.9 & & 62.1 & & FA040 \\
\hline 82 & 0.62 & 1.33 & 9.4 & 26.5 & 670 & 11.2 & 57.9 & $<0.002$ & 0.02 & 1.06 & & 1.60 & 209.0 & \begin{tabular}{|l|}
0.71 \\
\end{tabular} & $<0.05$ & 7.3 & 0.402 & 0.34 & 2.0 & 112 & 1.0 & 14.9 & 74 & 58.1 & & FA04030732 \\
\hline & & 1.27 & 10.0 & 25.4 & 870 & 11.1 & 55.1 & $<0.002$ & 0.02 & 1.22 & & 1.70 & 212.0 & 0.75 & $<0.05$ & 9.0 & 442 & 0.32 & 2.7 & 126 & & 17. & & 72. & & \\
\hline & 0.71 & 1.26 & 9.7 & 25.8 & 780 & 11.3 & 56.1 & $<0.002$ & 0.03 & 1.18 & & 1.70 & 203.0 & 0.73 & $<0.05$ & 8.5 & .419 & 0.35 & 2.4 & 118 & 1.1 & 16. & & 68.4 & & $\overline{F A 040}$ \\
\hline 85 & 0.76 & 1.47 & 10.4 & 31.2 & 800 & 11.6 & 58.0 & $<0.002$ & 0.02 & 1.18 & & 1.70 & 243.0 & 0.82 & $<0.05$ & 9.2 & 460 & 0.34 & 2.2 & 1 & 1.0 & 17.5 & & 68.4 & & FA04030732 \\
\hline & & 1.33 & 8.5 & 31.8 & 840 & 13.0 & 60.5 & $<0.002$ & 0.03 & 1.38 & & & 219.0 & 0.61 & 0.05 & 8.6 & & 0.35 & 2.2 & 19 & & 17.7 & 84 & 60.4 & & \\
\hline 87 & 0.77 & 1.36 & 9.8 & 30.4 & 850 & 11.7 & 53.6 & $<0.002$ & 0.02 & 1.29 & & 1.60 & 227.0 & 0.73 & $<0.05$ & 8.5 & 0.434 & 0.30 & 2.3 & 120 & 1.0 & 18.4 & 74 & 69.0 & & FA04030732 \\
\hline 1605 & 0.80 & 1.13 & 10.4 & 24.6 & 690 & 13.1 & 70.4 & $<0.002$ & 0.05 & 0.77 & & 2.10 & 159.5 & 0.78 & $<0.05$ & 8.1 & 0.461 & 0.43 & 2.0 & 120 & 1.2 & 14.2 & 62 & 69.1 & & FA04030732 \\
\hline & & & 10.4 & & 600 & 13.4 & 70.3 & $<0.002$ & 0.04 & 0.83 & & & & 0.77 & 0.05 & 8.1 & & & 2.0 & & & & & 66.6 & & FA04030732 \\
\hline 07 & 0.76 & 1.38 & 10.6 & 27.2 & 640 & 12.7 & 70.3 & $<0.002$ & 0.04 & 0.87 & & 2.00 & 192.0 & 0.80 & 0.05 & 8.8 & 0.472 & 0.40 & 2.0 & 126 & 1.3 & 15.0 & & 67.3 & & FA04030732 \\
\hline 1608 & 1.06 & \begin{tabular}{|l|l|}
1.11 \\
\end{tabular} & 10.6 & 24.2 & 580 & 12.3 & 60.5 & $<0.002$ & 0.03 & 1.02 & & 2.00 & 161.5 & 0.77 & $<0.05$ & 9.3 & 0.475 & 0.37 & 2.2 & 120 & 1.3 & 13.9 & 59 & 67.4 & & FA04030732 \\
\hline & & & & 23.6 & 540 & 12.7 & 58.7 & & 0.03 & & & & & 0.77 & $<0.05$ & 10.0 & & & & 124 & & & & 710 & & FA04030732 \\
\hline 1610 & 1.11 & 0.92 & 11.2 & 29.5 & 690 & 14.5 & 70.9 & $<0.002$ & 0.04 & 1.30 & 1 & 2.30 & 143.5 & 0.82 & 0.05 & 8.0 & 0.454 & 0.45 & 1.9 & 140 & 1.2 & 13.1 & 79 & 64.8 & & FA04030732 \\
\hline 1611 & 0.76 & 1.17 & 11.5 & 23.0 & 500 & 11.3 & 54.3 & $<0.002$ & 0.02 & 0.96 & & 1.80 & 166.5 & 0.84 & $<0.05$ & 10.4 & 0.515 & 0.32 & 2.2 & 116 & 1. & 14.9 & 57 & 73.0 & & A04030732 \\
\hline & 0.82 & 1.16 & & 24.5 & 560 & 12.7 & 61. & & 0.04 & 0.89 & & 1.80 & & 0.77 & & 7.9 & & & 1.9 & & & & & 64.0 & & FA04030732 \\
\hline 1614 & 0.80 & 1.16 & 10.6 & 24.3 & 530 & 11.8 & 54.8 & $<0.002$ & 0.02 & 0.98 & 1 & 1.90 & 174.0 & \begin{tabular}{|l|}
0.79 \\
\end{tabular} & $<0.05$ & 11.0 & 0.558 & 0.34 & 2.1 & 129 & 1.4 & 15.4 & 63 & 67.6 & 35 & FA04030732 \\
\hline 1615 & 1.13 & 1.00 & \begin{tabular}{|l|l|}
11.4 \\
\end{tabular} & 25.6 & 680 & 14.0 & 67.5 & $<0.002$ & 0.04 & 2.22 & & 2.10 & 167.0 & 0.81 & 0.05 & 8.3 & 0.449 & 0.41 & 2.0 & 133 & 1.2 & 14.2 & 67 & 66.2 & $\overline{22}$ & FA04030732 \\
\hline
\end{tabular}


Table 1. Location and New Trace Element Geochemical Results for 902 Stream-Sediment Samples collected in the Livengood Area, Livengood Quadrangle, Alaska IS = Insufficient Sample for Analysis

\begin{tabular}{|c|c|c|c|c|c|c|c|c|c|c|c|c|c|c|c|c|c|c|c|c|c|c|c|c|c|c|c|}
\hline AMPLE & UTM E & TM N & $\begin{array}{l}\mathrm{Au} \\
\mathrm{ppm}\end{array}$ & $\begin{array}{c}\mathrm{Ag} \\
\mathrm{ppm}\end{array}$ & \begin{tabular}{|l|} 
Al \\
$\%$ \\
\end{tabular} & $\begin{array}{l}\text { As } \\
\mathrm{ppm}\end{array}$ & $\begin{array}{l}\mathrm{Ba}^{*} \\
\mathrm{ppm}\end{array}$ & $\begin{array}{c}\mathrm{Be} \\
\mathrm{ppm}\end{array}$ & $\begin{array}{c}\mathrm{Bi} \\
\mathrm{ppm}\end{array}$ & $\begin{array}{c}\mathrm{Ca} \\
\%\end{array}$ & \begin{tabular}{|c|}
$\mathrm{Cd}$ \\
$\mathrm{ppm}$ \\
\end{tabular} & $\begin{array}{c}\mathrm{Ce} \\
\mathrm{ppm}\end{array}$ & $\begin{array}{l}\text { Co } \\
\text { ppm }\end{array}$ & $\begin{array}{l}\mathrm{Cr}^{\star} \\
\mathrm{ppm}\end{array}$ & $\begin{array}{c}\text { Cs } \\
\text { ppm }\end{array}$ & $\begin{array}{c}\mathrm{Cu} \\
\mathrm{ppm}\end{array}$ & $\begin{array}{c}\mathrm{Fe} \\
\% \\
\end{array}$ & $\begin{array}{c}\mathrm{Ga} \\
\mathrm{ppm}\end{array}$ & $\begin{array}{c}\mathrm{Ge} \\
\mathrm{ppm}\end{array}$ & $\begin{array}{c}\mathrm{Hf} \\
\mathrm{ppm}\end{array}$ & $\begin{array}{l}\mathrm{Hg} \\
\mathrm{ppm}\end{array}$ & $\begin{array}{c}\text { In } \\
\text { ppm }\end{array}$ & $\begin{array}{l}\mathrm{K} \\
\%\end{array}$ & $\begin{array}{c}\mathrm{La} \\
\mathrm{ppm}\end{array}$ & \begin{tabular}{|c|}
$\mathrm{Li}$ \\
$\mathrm{ppm}$
\end{tabular} & $\begin{array}{c}\mathrm{Mg} \\
\%\end{array}$ & $\begin{array}{l}\mathrm{Mn} \\
\mathrm{ppm}\end{array}$ \\
\hline 1617 & 99961 & 51467 & 0.005 & 0.13 & 6.53 & 81 & 800 & & 0.14 & 1.36 & 0.17 & & 105 & & 3.03 & & 2.91 & & & \begin{tabular}{|l|}
2.1 \\
\end{tabular} & 0.22 & & & & 28.4 & & 378 \\
\hline 1618 & 737 & 51287 & .005 & 09 & 6.12 & & 10 & 10 & 0.10 & 1.34 & 0.13 & 71.2 & 10.4 & & 2.59 & 0.8 & 3.10 & 4.35 & 0.21 & 1.8 & 0.15 & 0.044 & 1.13 & 34.1 & 25.1 & 0.92 & 452 \\
\hline & & & 005 & & 5.92 & 6.9 & 710 & $\overline{06}$ & & & 0.20 & & 11.7 & 20 & & $\sqrt{41}$ & 2.95 & 14.35 & 0.19 & 2.0 & 0.32 & 0.044 & \begin{tabular}{|l|}
1.10 \\
\end{tabular} & 35.2 & 25.9 & 0.89 & 25 \\
\hline 62 & & & & & \begin{tabular}{|l|}
6.33 \\
\end{tabular} & 15.0 & 770 & & 0. & & & 72 . & 16 & & & 3. & 3.32 & 15.75 & 0.21 & 1.8 & 089 & & \begin{tabular}{|l|}
1.27 \\
\end{tabular} & 34.4 & \begin{tabular}{|l|}
489 \\
\end{tabular} & 0.76 & 10 \\
\hline$\overline{16}$ & & & .006 & 27 & \begin{tabular}{|l|}
6.48 \\
\end{tabular} & 7.9 & 790 & $\overline{08}$ & 0.15 & .04 & 31 & 5.9 & 14.1 & 90 & .90 & 21.6 & 3.09 & 16.40 & 0.19 & 1.9 & 0.34 & .053 & 1.23 & 32.2 & 5.3 & 0.88 & 729 \\
\hline & & & & & & & 440 & & & & & & & & & & 3.50 & & & 21 & & & & & & 098 & \\
\hline 1625 & 365 & 49340 & 0.005 & .22 & \begin{tabular}{|l|l|}
6.44 \\
\end{tabular} & 7.9 & 740 & 1.16 & 0.14 & 1.25 & \begin{tabular}{|l|}
0.42 \\
\end{tabular} & 76.7 & 17.4 & 8 & 4.40 & 26.5 & 3.21 & 15.75 & 0.22 & 1.9 & 0.38 & 0.055 & 1.20 & 36.8 & 44.4 & 0.99 & 1215 \\
\hline 1626 & & & 007 & 0.16 & 6.25 & 6.4 & 720 & 1.14 & 0.14 & 1.13 & 0.29 & 73.9 & 17.1 & 9 & 3.69 & 23.0 & 3.07 & 15.30 & 0.20 & 2.1 & 1.33 & .050 & \begin{tabular}{|l|}
1.21 \\
\end{tabular} & 34.2 & \begin{tabular}{|l|l|}
41.4 \\
\end{tabular} & 0.97 & 996 \\
\hline & & & & & 5.74 & & & & & & & & & & & & & & 0.20 & 2.0 & & & & 37.8 & & 0.92 & \\
\hline 1629 & & & 0.005 & 0.49 & 7.42 & 15.0 & 870 & 1.44 & 0.21 & 0.85 & .80 & 80.0 & 44.2 & 100 & 7.45 & 50.9 & 4.22 & 18.95 & 0.23 & 2.3 & 0.64 & 0.072 & 1.44 & 34.4 & 53.0 & 0.99 & 2830 \\
\hline 164 & & & .005 & 0.13 & 6.01 & 7.9 & 890 & 1.22 & 0.10 & 1.92 & 0.32 & 71.5 & 15.3 & 7 & 2.36 & 18.6 & 3.90 & 15.40 & 0.23 & 2.5 & 0.16 & 0.051 & 1.11 & 34.6 & \begin{tabular}{|l|}
22.1 \\
\end{tabular} & $\begin{array}{l}1.10 \\
\end{array}$ & 748 \\
\hline $164 \varepsilon$ & & & .032 & 13 & 6.21 & & 900 & 1.14 & & 1.94 & & 82.6 & 14.8 & & 241 & 246 & 4.19 & 15.65 & 0.25 & 3.0 & & & \begin{tabular}{|l|}
1.08 \\
\end{tabular} & & \begin{tabular}{|l|}
22.7 \\
\end{tabular} & $\begin{array}{l}1.14 \\
\end{array}$ & \\
\hline$\overline{16}$ & & & 0.013 & 0.18 & 6.18 & 7.5 & 1000 & 1.18 & 0.11 & 1.88 & 0.35 & 74.3 & 13. & 8 & 2.7 & 28. & 3.61 & 15.00 & 0.24 & 2.5 & 0.23 & .048 & 1.19 & 35.7 & 24.3 & 1.13 & 698 \\
\hline 16 & & & .007 & $\overline{12}$ & \begin{tabular}{|l|}
6.14 \\
\end{tabular} & 7. & 920 & .20 & 0.10 & 1.79 & 0.24 & 78. & 15. & & 2.40 & 20. & 3.76 & 14.75 & 0.22 & 2.6 & 0.10 & .049 & \begin{tabular}{|l|}
1.13 \\
\end{tabular} & 37.8 & 23.0 & 1.08 & 782 \\
\hline & & & .005 & 12 & & & 90 & & & $\overline{93}$ & & & 1 & & & & 3.72 & 4.80 & .22 & & & 051 & & 99.3 & 3.3 & & 43 \\
\hline 16 & & & 0.005 & 0.11 & 5.79 & 7.1 & 890 & 1.16 & 0.10 & 1.92 & 0.25 & 70.8 & 11.4 & 7 & 2.21 & 17.2 & 3.00 & 13.95 & 0.19 & 2.2 & 0.19 & 0.044 & \begin{tabular}{|l|}
1.19 \\
\end{tabular} & 34.3 & \begin{tabular}{|l|}
21.8 \\
\end{tabular} & \begin{tabular}{|l|}
1.02 \\
\end{tabular} & 530 \\
\hline & & & 006 & 10 & \begin{tabular}{|l|}
6.10 \\
\end{tabular} & & 940 & & & 1.88 & 5 & 90.2 & 14.2 & & 2.60 & 21.8 & 3.53 & 15.20 & 0.23 & 2. & 0.13 & .053 & \begin{tabular}{|l|}
1.22 \\
\end{tabular} & 43.9 & 24.6 & \begin{tabular}{|l|l} 
\\
\end{tabular} & 761 \\
\hline & & & .005 & 13 & \begin{tabular}{|l|}
6.31 \\
\end{tabular} & 5. & 1030 & 1.26 & 0.10 & 1.98 & 0.43 & 72. & 14. & & 2.4 & 33. & 3.22 & 15.05 & 0.20 & 2.4 & 0 & 050 & \begin{tabular}{|l|}
1.21 \\
\end{tabular} & 34.5 & 23.6 & 1.14 & 15 \\
\hline 16 & & & .005 & .11 & \begin{tabular}{|l|l}
5.83 \\
\end{tabular} & & 910 & 20 & 100 & 1.89 & .27 & 62 & 11 & & 22 & 17. & 2.85 & 13.50 & 0.18 & 1. & 0.2 & 043 & \begin{tabular}{|l|}
1.23 \\
\end{tabular} & 30.1 & 22.7 & $\overline{0.99}$ & $\overline{502}$ \\
\hline$\overline{16}$ & & & 009 & 13 & & & & & & 88 & & & & & & & 3.47 & 4.50 & 0. & & $\bar{c}$ & 050 & 1.16 & 44.7 & 3.6 & 1.04 & 90 \\
\hline$\overline{16}$ & & & .005 & 0.14 & \begin{tabular}{|l}
6.02 \\
\end{tabular} & 8.2 & 950 & $1.1 \varepsilon$ & 0.10 & 1.85 & .32 & 80.4 & 13. & 8 & 2.52 & 24.0 & 3.57 & 14.65 & 0.23 & 2.6 & 0.18 & 0.050 & \begin{tabular}{|l}
1.19 \\
\end{tabular} & 38.6 & 24.1 & 1.06 & 644 \\
\hline 16 & & & .005 & 12 & \begin{tabular}{|l|}
5.30 \\
\end{tabular} & 12.2 & 860 & & & 1.81 & $E$ & 65 & 14. & & 2.2 & 17.8 & 3.35 & 12.55 & 0.18 & 2.1 & 0.22 & .039 & \begin{tabular}{|l|}
1.09 \\
\end{tabular} & 31.5 & \begin{tabular}{|l|}
22.1 \\
\end{tabular} & 0.86 & 1290 \\
\hline & & & .005 & 13 & \begin{tabular}{|l|}
6.12 \\
\end{tabular} & 8.5 & 980 & & & 85 & & & 1 & & 2.6 & & 3.32 & 4.95 & & & 0.15 & .048 & 1.22 & 32.4 & 4.6 & 1.04 & 682 \\
\hline$\overline{16}$ & & & .005 & 0.12 & 5.91 & 7. & 920 & 1.21 & 0.10 & 1.99 & 0.24 & 63.7 & 11. & & 2.01 & 19. & 3.30 & 13.40 & 0.17 & 2.7 & 0.10 & .043 & \begin{tabular}{|l|}
1.14 \\
\end{tabular} & 32.5 & 20.3 & 1.08 & 598 \\
\hline 166 & & & 007 & 14 & 5.86 & 8. & 910 & 1.4 & & 1.74 & 3. & 67. & 12 . & & 2.4 & 24. & 3.25 & 3.95 & 0.15 & 2. & 0.07 & .047 & \begin{tabular}{|l|}
1.21 \\
\end{tabular} & 34.1 & 25.0 & 1.02 & 637 \\
\hline & & & & & 5.99 & 7. & 820 & & & 1.94 & & & & & & & & & & 2. & & & & & 3.6 & 1.1 & 26 \\
\hline & & & $<0.005$ & 11 & \begin{tabular}{|l}
6.02 \\
\end{tabular} & & 800 & 1.2 & & 2.01 & & & 11 & & 2.2 & 22. & 3.19 & 14.70 & 02 & 3. & 0.1 & 0.046 & \begin{tabular}{|l|}
1.12 \\
\end{tabular} & 44.9 & \begin{tabular}{|l|}
23.4 \\
\end{tabular} & 1.12 & 525 \\
\hline 16 & & & .005 & 10 & 6.12 & 4. & 850 & & & 1.76 & & & 10 & & 2.4 & 2. & 2.87 & 4.50 & & & 2 & 044 & 1.21 & 34.0 & 4.3 & 1.0 & 404 \\
\hline & & & & & & & 860 & & & & & & & & 2.24 & 22.2 & 3.19 & & & & & & & 30.7 & & & 508 \\
\hline 167 & & & 0.005 & 15 & \begin{tabular}{|l|l|}
5.87 \\
\end{tabular} & 6. & 870 & & & 1.72 & $\overline{1.16}$ & 74. & 11. & & 2.3 & 21.8 & 3.01 & 3.80 & 0.2 & 2.6 & 0.13 & .046 & 1.20 & 37.1 & 24.3 & 1.00 & 456 \\
\hline & & & .005 & 15 & 6.07 & 8. & 890 & 1. & & 1.66 & & & 11 & & & 23. & 3.13 & 4.25 & & 2. & - & & 1.24 & 29.7 & 24.7 & 0.98 & 522 \\
\hline & & & 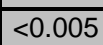 & & & & & & & & & & & & & & & & & & & & & & & 10 & 585 \\
\hline 1675 & & & 0.019 & .08 & 5.79 & 7.1 & 790 & 1. & & 1.98 & 21 & 105.5 & 13. & & 1.99 & 0.3 & 3.50 & 3.75 & 0.23 & 3.5 & 0.09 & .048 & 1.08 & 50.4 & 21.2 & 1.10 & $\overline{666}$ \\
\hline & & & .005 & 0.15 & \begin{tabular}{|l}
6.04 \\
\end{tabular} & 7.4 & 870 & & 0. & 2.01 & 0. & 72.2 & 12 & 84 & 2.21 & $\overline{15}$ & 3.29 & 4.60 & 0. & 2.6 & 0. & 046 & 1.19 & 35.7 & 22.5 & 1.09 & 584 \\
\hline & & & & & & 7 & & & & & & & & & 2.2 & & 3.20 & & & & & 042 & & 36.2 & 23.5 & & 573 \\
\hline 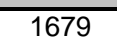 & & 33 & 0.005 & 0.22 & \begin{tabular}{|l|l|}
6.29 \\
\end{tabular} & 10.3 & 1030 & 1.25 & 0. & 1.22 & \begin{tabular}{|l|}
0.57 \\
\end{tabular} & 64 & 12 . & 12 & 3.7 & 32. & 3.16 & 14.65 & 0.19 & 2.8 & 0.18 & 0.048 & \begin{tabular}{|l|}
1.42 \\
\end{tabular} & 31.6 & 30.3 & 1.05 & 666 \\
\hline & & & & & & 8.7 & 980 & & & 1.94 & & & & & & & 2.95 & & & 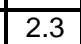 & & & \begin{tabular}{|l|}
1.34 \\
\end{tabular} & 8.0 & 22.5 & 1.07 & 66 \\
\hline & & & .005 & & & 8 & 980 & & & & & & & & 4.0 & & 2.78 & & & 2. & & .049 & 1.40 & 29.1 & 27.0 & 0.93 & 500 \\
\hline 1684 & & 7290 & $<0.005$ & 0.20 & \begin{tabular}{|l|l}
6.09 \\
\end{tabular} & 9. & 970 & 1.26 & $\overline{0.1}$ & 1.20 & \begin{tabular}{|l}
0.46 \\
\end{tabular} & 75.0 & 11. & 9 & 3.3 & 31. & 2.89 & 14.45 & 0.20 & 2.9 & .17 & 0.048 & 1.34 & 34.8 & 24.5 & 0.88 & 472 \\
\hline & & & 0.005 & & \begin{tabular}{|l|l|}
6.11 \\
\end{tabular} & & 940 & & & 1.29 & & & & & $\overline{-}$ & 27. & 2.63 & & & 2. & 0.2 & $0<$ & 1.37 & 27.5 & 22.5 & 0.86 & 454 \\
\hline 1686 & & & 0.016 & 13 & & 6.9 & 91 & & $\overline{0 .}$ & 1. & & $\overline{9}$ & & & 2.8 & & 2.8 & & & 3.7 & & & & 45.5 & 1 & 0.92 & 517 \\
\hline $168 \varepsilon$ & & & $=080$ & 0.19 & \begin{tabular}{|l|l}
6.18 \\
\end{tabular} & 7.2 & 970 & 1.02 & 0. & 1.27 & \begin{tabular}{|l|}
0.56 \\
\end{tabular} & 56 & 12 . & 81 & 3.2 & 28.7 & 2.76 & 4.30 & .20 & 2.3 & 0.20 & .04 & 1.34 & 27.6 & 1. & 0.85 & 651 \\
\hline & & & & & & & & & & & & & & & & & & & & & & & & & & & \\
\hline
\end{tabular}


Table 1. Location and New Trace Element Geochemical Results for 902 Stream-Sediment Samples collected in the Livengood Area, Livengood Quadrangle, Alaska IS = Insufficient Sample for Analysis

\begin{tabular}{|c|c|c|c|c|c|c|c|c|c|c|c|c|c|c|c|c|c|c|c|c|c|c|c|c|c|c|}
\hline SAMPLE & $\begin{array}{l}\text { Mo } \\
\text { ppm }\end{array}$ & $\begin{array}{c}\mathrm{Na} \\
\%\end{array}$ & $\begin{array}{l}\mathrm{Nb} \\
\mathrm{ppm}\end{array}$ & $\begin{array}{r}\mathrm{Ni} \\
\mathrm{ppm}\end{array}$ & $\begin{array}{c}\mathrm{P} \\
\mathrm{ppm}\end{array}$ & $\begin{array}{l}\mathrm{Pb} \\
\mathrm{ppm}\end{array}$ & $\begin{array}{l}\mathrm{Rb} \\
\mathrm{ppm}\end{array}$ & $\begin{array}{l}\mathrm{Re} \\
\mathrm{ppm}\end{array}$ & $\begin{array}{l}\mathrm{S} \\
\%\end{array}$ & $\begin{array}{l}\text { Sb } \\
\text { ppm }\end{array}$ & $\begin{array}{c}\text { Se } \\
\text { ppm }\end{array}$ & $\begin{array}{l}S^{S} n^{*} \\
\text { ppm }\end{array}$ & $\begin{array}{c}\mathrm{Sr} \\
\mathrm{ppm}\end{array}$ & $\begin{array}{l}\mathrm{Ta}^{*} \\
\mathrm{ppm}\end{array}$ & $\begin{array}{c}\text { Te } \\
\text { ppm }\end{array}$ & $\begin{array}{c}\text { Th } \\
\text { ppm }\end{array}$ & $\begin{array}{l}\mathrm{Ti}^{\star} \\
\% \\
\end{array}$ & $\begin{array}{c}\mathrm{Tl} \\
\mathrm{ppm}\end{array}$ & $\begin{array}{c}U \\
\mathrm{ppm}\end{array}$ & $\begin{array}{c}\mathrm{V} \\
\mathrm{ppm}\end{array}$ & $\begin{array}{l}W^{*} \\
\text { ppm }\end{array}$ & $\begin{array}{c}\mathrm{Y} \\
\mathrm{ppm}\end{array}$ & $\begin{array}{l}\mathrm{Zn} \\
\mathrm{ppm}\end{array}$ & $\begin{array}{l}\mathrm{Zr}^{*} \\
\mathrm{ppm}\end{array}$ & $\begin{array}{c}\text { Pulp Wt } \\
\text { grams }\end{array}$ & $\begin{array}{c}\text { Lab } \\
\text { Report }\end{array}$ \\
\hline 1617 & 0.85 & 1.27 & 11.2 & 26.2 & 540 & 13.7 & 65.3 & $<0.002$ & 0.03 & 1.06 & 1 & 2.10 & 195.0 & 0.84 & $<0.05$ & 9.1 & 0.489 & 0.41 & 2.2 & 127 & 1.1 & 15.0 & 66 & 70.3 & 26 & FA04030732 \\
\hline 1618 & 0.76 & .26 & 9.5 & 23.6 & 510 & 12.0 & 58.2 & $<0.002$ & 0.03 & 0.92 & 1 & 1.90 & 185.5 & 0.72 & $<0.05$ & 9.3 & 0.453 & 0.35 & 2.0 & 120 & 1.0 & 14.4 & 63 & 62.0 & 22 & FA04030732 \\
\hline 1619 & 0.65 & 1.22 & 10.5 & 25.6 & 590 & 11.9 & 56.7 & $<0.002$ & 0.04 & 1.00 & & 2.00 & 184.5 & 0.80 & $<0.05$ & 9.6 & 0.480 & 0.33 & 2.1 & 119 & 1.0 & 15.6 & 69 & 68.8 & 27 & A04030732 \\
\hline 1621 & 1.33 & 0.92 & 1.8 & 31.5 & 770 & 13.9 & 71.8 & 0.002 & 0.05 & 2.57 & & 2.30 & 170.5 & 0.80 & $<0.05$ & 9.4 & 0.426 & 0.44 & 2.1 & 137 & 1.1 & 15.9 & 98 & 62.6 & 30 & FA04030732 \\
\hline 1622 & 1.00 & .01 & 10.0 & 30.8 & 750 & 14.0 & 70.3 & $<0.002$ & 0.04 & 0.90 & 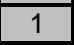 & 2.10 & 167.0 & 0.74 & $<0.05$ & 8.2 & 0.457 & 0.56 & 2.0 & 138 & 1.2 & 18.0 & 82 & 64.7 & 19 & FA04030732 \\
\hline 1624 & 1.27 & 0.99 & 11.0 & 40.6 & 770 & 14.7 & 81.0 & $<0.002$ & 0.06 & 0.99 & 2 & 2.20 & 169.5 & 0.77 & 0.07 & 9.5 & 0.444 & 0.57 & 2.4 & 154 & 1.2 & 21.8 & 104 & 74.0 & 26 & FA04030732 \\
\hline 1625 & 0.87 & 1.03 & $\overline{0.2}$ & 38.0 & 720 & 13.1 & 69.4 & $<0.002$ & 0.09 & 0.83 & & 1.90 & 174.5 & 0.73 & 0.06 & 8.4 & 0.431 & 0.45 & 2.3 & 136 & 1.0 & 20.9 & 106 & 67.8 & 17 & FA04030732 \\
\hline 1626 & 0.78 & 1.12 & 10.2 & 37.5 & 610 & 11.3 & 64.3 & $<0.002$ & 0.05 & 0.81 & 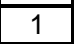 & 1.80 & 169.0 & 0.74 & 0.05 & 8.6 & 0.449 & 0.41 & 2.3 & 134 & 1.0 & \begin{tabular}{|l|}
18.6 \\
\end{tabular} & 93 & 69.9 & & FA04030732 \\
\hline 1627 & 0.51 & 1.32 & 10.8 & 29.4 & 480 & 9.0 & 51.6 & $<0.002$ & 0.02 & 0.76 & & 1.70 & 181.0 & 0.81 & $<0.05$ & 9.1 & 0.507 & 0.30 & 2.1 & 118 & 1.0 & 16.1 & 77 & 67.9 & $4 \varepsilon$ & FA04030732 \\
\hline 162 & & 0.86 & 9.7 & 42.7 & 1100 & 19.7 & 91.6 & $<0.002$ & 0.07 & 1.33 & & 2.30 & 142.0 & 0.69 & \begin{tabular}{|l|}
0.10 \\
\end{tabular} & 8.0 & 0.422 & 0.55 & 2.3 & 180 & 1. & 21.4 & 183 & 80.3 & & FA04030732 \\
\hline 1646 & 1.04 & 1.47 & 13.3 & 27.9 & 800 & 11.3 & 52.7 & $<0.002$ & 0.04 & 0.97 & & 1.80 & 235.0 & 0.94 & 0.07 & 8.6 & 0.754 & 0.31 & 2.2 & 176 & 1.4 & 19.7 & 88 & 89.0 & & 0732 \\
\hline 1648 & 1.19 & 1.41 & 16.0 & 29.2 & 800 & 11.3 & 52.2 & $<0.002$ & 0.03 & 0.95 & & 2.00 & 230.0 & 1.12 & 0.06 & 10.2 & 0.952 & 0.34 & 2.7 & 196 & 3.2 & 20.7 & 85 & 109.5 & 2 & FA04030732 \\
\hline $164 !$ & & 1.34 & 2.2 & 30.1 & 800 & 12.1 & 59.0 & $<0.002$ & 0.04 & 1.30 & & 1.80 & 226.0 & 0.88 & $<0.05$ & 9.0 & 0.606 & 0.36 & 2.5 & & 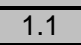 & \begin{tabular}{|l|}
19.2 \\
\end{tabular} & & 85.1 & & \\
\hline 165 & 1.10 & 1.38 & 13.2 & 27.5 & 790 & 11.6 & 52.7 & $<0.002$ & 0.03 & 0.96 & 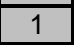 & 1.80 & 227.0 & 0.94 & $<0.05$ & 10.0 & 0.708 & 0.34 & 2.6 & 160 & 1.6 & 19.5 & 78 & 91.9 & & 0732 \\
\hline 1652 & 1.16 & 1.36 & 13.4 & 28.5 & 820 & 11.3 & 55.5 & $<0.002$ & 0.03 & 1.02 & & 1.80 & 227.0 & 0.95 & 0.05 & 10.0 & 0.686 & 0.35 & 2.6 & 160 & 1.4 & 20.0 & 83 & 96.6 & 3 & 30732 \\
\hline 1653 & .99 & 1.43 & 10.8 & 26.7 & 790 & 10.8 & 53.0 & $<0.002$ & 0.02 & 1.14 & & .60 & 243.0 & 0.78 & $<0.05$ & 8.9 & 0.462 & 0.32 & 2.3 & & 1.0 & 18.1 & & 75.4 & & \\
\hline 1655 & 1.09 & 1.37 & 13.4 & 30.0 & 860 & 11.9 & 59.7 & $<0.002$ & 0.03 & 1.07 & & 1.80 & 233.0 & 0.95 & 0.06 & 11.0 & 0.619 & 0.37 & 2.9 & $14 \varepsilon$ & 1.8 & 21.4 & 8 & 100.5 & 4 & 0732 \\
\hline 165 & 1.13 & 1.44 & 11.2 & 31.8 & 790 & 12.1 & 57.3 & $<0.002$ & 0.03 & 1.10 & & 1.70 & 238.0 & 0.84 & $<0.05$ & \begin{tabular}{l|l|}
8.5 \\
\end{tabular} & 0.548 & 0.35 & 2.4 & 14 & 1. & 19.2 & & 82.6 & & 732 \\
\hline 1658 & 0.83 & 1.42 & 9.6 & 27.3 & 810 & 10.9 & 55.1 & $<0.002$ & 0.03 & 1.04 & & 1.50 & 242.0 & 0.70 & $<0.05$ & 7.8 & 0.396 & 0.31 & 2.0 & & 0. & \begin{tabular}{|l|}
16.4 \\
\end{tabular} & 7 & 66. & & 0732 \\
\hline 1659 & 1.14 & 1.30 & 13.1 & 29.4 & 870 & 11.7 & 56.8 & $<0.002$ & 0.03 & 1.06 & & 1.80 & 226.0 & 0.97 & 0.05 & 11.4 & 0.595 & 0.35 & 2.8 & 143 & 2.3 & 21.0 & 83 & 98.7 & 4 & 30732 \\
\hline 1660 & 1.16 & 1.36 & 12.0 & 29.2 & 850 & 11.5 & 56.6 & $<0.002$ & 0.03 & 1.07 & & 1.70 & 230.0 & 0.87 & $<0.05$ & 9.6 & 0.554 & 0.33 & 2.6 & 14 & 1.5 & 20.0 & 82 & 91.4 & & 0732 \\
\hline 1661 & 1.18 & 1.13 & 9.8 & 28.2 & 1060 & 12.6 & 58.3 & $<0.002$ & 0.06 & 1.17 & & 1.50 & 208.0 & 0.70 & \begin{tabular}{|l|}
0.05 \\
\end{tabular} & 8.9 & 0.396 & 0.33 & 2.2 & 11 & 1. & 16.8 & 102 & 72 & & 732 \\
\hline 1662 & 1.11 & 1.38 & 10.8 & 30.4 & 820 & 12.2 & 60.2 & $<0.002$ & 0.03 & 1.14 & & 1.70 & 233.0 & 0.78 & $<0.05$ & 8.7 & 0.456 & 0.36 & 2.3 & 13 & 1. & 18.6 & 85 & 77.8 & & FA04030732 \\
\hline 1664 & 0.97 & 1.44 & 11.2 & 27.1 & 820 & 10.2 & 48.7 & 0.002 & 0.02 & 0.98 & & 1.50 & 234.0 & 0.84 & $<0.05$ & 8.9 & 0.534 & 0.31 & 2.3 & 1 & 1. & 17.3 & & 79.8 & & 732 \\
\hline 1665 & 1.04 & 1.31 & 11.5 & 29.5 & 770 & 11.2 & 56.1 & 0.003 & 0.03 & 1.07 & & 1.60 & 218.0 & 0.85 & 0.05 & 9.3 & 0.502 & 0.38 & 2.4 & 134 & 1.2 & 17.6 & 86 & 75.3 & 3 & 0732 \\
\hline 1666 & 0.85 & 1.35 & 12.2 & 30.3 & 830 & 11.2 & 51.9 & $<0.002$ & 0.03 & 0.93 & & 1.60 & 230.0 & 0.89 & $<0.05$ & 9.5 & 0.596 & 0.33 & 2.6 & 14 & 1.2 & 18.8 & 8 & 83.6 & 4 & 0732 \\
\hline 16 & 0.69 & 1.40 & 13.9 & 29.0 & 810 & & 52.6 & $<0.002$ & 0.02 & 0.90 & & 1. & 235.0 & 1.03 & $<0.05$ & 12.0 & 0.682 & 0.32 & 2.8 & 1 & & 20.0 & 7 & 94 & & \\
\hline 1668 & 0.67 & 1.40 & 11.6 & 26.8 & 790 & 11.2 & 54.1 & $<0.002$ & 0.03 & 0.84 & & 1.70 & 231.0 & \begin{tabular}{|l|l|}
0.84 \\
\end{tabular} & $<0.05$ & 9.7 & 0.531 & 0.38 & 2.4 & 13 & 1. & 17.3 & 7 & 77. & & 0732 \\
\hline 1670 & 0.81 & 1.32 & 10.0 & 26.6 & 750 & 10.5 & 50.2 & $<0.002$ & 0.03 & 1.01 & & 1.50 & 221.0 & 0.72 & $<0.05$ & 8.2 & 0.478 & 0.33 & 2.1 & 13 & 1. & 15.8 & 7 & 66.7 & & 7732 \\
\hline & 0.75 & 1.31 & 11.8 & 27.3 & 790 & 11.6 & 57.3 & $<0.002$ & 0.03 & 0.98 & & 70 & 220.0 & 0.86 & $<0.05$ & 10.4 & 0.505 & 0.38 & 2.5 & & & 17.7 & 74 & 80.7 & & \\
\hline 1672 & 0.99 & 1.33 & 9.4 & 28.6 & 790 & 11.6 & 59.5 & 0.002 & 0.04 & 1.03 & & 1.60 & 220.0 & \begin{tabular}{|l|}
0.72 \\
\end{tabular} & $<0.05$ & 8.2 & 0.395 & 0.37 & 2.2 & & 1. & 16.0 & 8 & 63.1 & & 7732 \\
\hline 1673 & 0.87 & 1.40 & 10.4 & 27.5 & 780 & 11. & 53.9 & $<0.002$ & 0.03 & 0.97 & & 1.50 & 230.0 & 0.75 & $<0.05$ & 8.2 & 0.478 & 0.36 & 2.1 & 1 & & 16.2 & & 68.0 & & $\overline{7732}$ \\
\hline & & 1.39 & & 27.0 & 840 & & 47.9 & $<0.002$ & 0.02 & 0.91 & & 1.60 & 234.0 & 0.99 & & 14.1 & & 0.30 & 3.0 & & & 20.8 & & 102.5 & & \\
\hline 1676 & 0.84 & 1.47 & 12.0 & 29.5 & 810 & 11.2 & 54.7 & 0.002 & 0.02 & 1.00 & & 1.60 & 244.0 & 0.82 & $<0.05$ & 10.1 & 0.548 & 0.36 & 2.5 & $13 \varepsilon$ & 1. & 18.2 & 82 & 78.1 & 5 & FA04030732 \\
\hline 1677 & 0.77 & 1.39 & 11.7 & 28.3 & 850 & 11.0 & 54.7 & $<0.002$ & 0.02 & 1.00 & & 1.60 & 233.0 & 0.85 & $<0.05$ & 10.4 & 0.528 & 0.34 & 2.6 & 136 & 1.5 & \begin{tabular}{|l|}
17.9 \\
\end{tabular} & 82 & 85.6 & 5 & 0732 \\
\hline & & 1.27 & 9.7 & 43.8 & 860 & & 65.7 & & & & & & & & & 8.5 & 0.427 & & & & & \begin{tabular}{|l|}
18.1 \\
\end{tabular} & & 84.3 & & \\
\hline 1681 & 1.06 & 1.43 & 9.5 & 30.1 & 860 & 10.8 & 56.3 & 0.003 & 0.03 & 1.09 & \pm & 1.40 & 246.0 & 0.69 & $<0.05$ & 7.7 & 0.380 & 0.43 & 2.2 & 122 & 1.0 & 16.6 & 86 & 71.3 & 79 & 0732 \\
\hline 1683 & 1.69 & 1.20 & 9.3 & 37.2 & 740 & 11.8 & 69.9 & 0.008 & 0.05 & 1.13 & & 1.60 & 185.5 & 0.67 & \begin{tabular}{|l|}
0.06 \\
\end{tabular} & 8.0 & 0.378 & 0.58 & 2.7 & 15 & 0.9 & 18.4 & 98 & 86.1 & 3 & \\
\hline 1684 & 1.80 & 1.22 & 10.6 & 35.1 & 760 & 11.3 & 65.0 & 0.007 & 0.04 & 1.20 & & 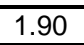 & 191.5 & 0.79 & 0.05 & 9.9 & 0.423 & 0.51 & 2.9 & & & \begin{tabular}{|l|}
19.1 \\
\end{tabular} & $g$ & 89.2 & & \\
\hline 1685 & 1.21 & 1.31 & 9.4 & 30.9 & 770 & 11.2 & 65.8 & 0.004 & 0.04 & 1.09 & 2 & 1.50 & 213.0 & 0.68 & 0.06 & 7.4 & 0.345 & 0.53 & 2.3 & 128 & 0.8 & \begin{tabular}{ll|}
18.5 \\
\end{tabular} & 86 & 68.9 & 29 & 30732 \\
\hline 1686 & 1.19 & 1.43 & 13.7 & 31.5 & 800 & 10.8 & 60.7 & 0.003 & 0.03 & 1.03 & & 1.80 & 229.0 & 0.99 & $<0.05$ & 11.7 & 0.532 & 0.47 & 3.0 & 13 & & \begin{tabular}{|l|}
21.2 \\
\end{tabular} & 8 & 110.0 & 35 & \\
\hline 1688 & 0.97 & 1.28 & 9.2 & 33.7 & 750 & 11.6 & 65.5 & 0.003 & 0.04 & 1.07 & 2 & 1.60 & 207.0 & 0.63 & \begin{tabular}{|l|}
0.06 \\
\end{tabular} & 7.6 & 0.348 & 0.54 & 2.4 & 12 & 1.0 & 17.8 & 94 & 66.3 & 25 & FA04030732 \\
\hline 1689 & 1.31 & 1.23 & 10.4 & 29.1 & 720 & 12.6 & 75.0 & $<0.002$ & 0.03 & 1.03 & & 1.90 & 235.0 & 0.75 & 0.05 & 8.4 & 0.399 & 0.46 & 2.4 & 133 & 1.3 & 15.8 & 81 & 70.6 & 20 & FA04030732 \\
\hline
\end{tabular}


Table 1. Location and New Trace Element Geochemical Results for 902 Stream-Sediment Samples collected in the Livengood Area, Livengood Quadrangle, Alaska IS = Insufficient Sample for Analysis

\begin{tabular}{|c|c|c|c|c|c|c|c|c|c|c|c|c|c|c|c|c|c|c|c|c|c|c|c|c|c|c|c|}
\hline MPLE & TM E & TM N & $\begin{array}{c}\mathrm{Au} \\
\mathrm{ppm}\end{array}$ & $\begin{array}{l}\mathrm{Ag} \\
\mathrm{ppm}\end{array}$ & $\begin{array}{l}\text { Al } \\
\%\end{array}$ & $\begin{array}{c}\text { As } \\
\text { ppm }\end{array}$ & $\begin{array}{l}\mathrm{Ba}^{*} \\
\mathrm{ppm}\end{array}$ & \begin{tabular}{|c|}
$\mathrm{Be}$ \\
$\mathrm{ppm}$
\end{tabular} & $\begin{array}{c}\mathrm{Bi} \\
\mathrm{ppm}\end{array}$ & $\begin{array}{c}\mathrm{Ca} \\
\%\end{array}$ & \begin{tabular}{|l|}
$\mathrm{Cd}$ \\
$\mathrm{ppm}$
\end{tabular} & $\begin{array}{c}\mathrm{Ce} \\
\mathrm{ppm}\end{array}$ & $\begin{array}{l}\text { Co } \\
\text { ppm }\end{array}$ & $\begin{array}{l}\mathrm{Cr}^{\star} \\
\mathrm{ppm}\end{array}$ & $\begin{array}{l}\text { Cs } \\
\text { ppm }\end{array}$ & $\begin{array}{l}\mathrm{Cu} \\
\mathrm{ppm}\end{array}$ & $\begin{array}{c}\mathrm{Fe} \\
\% \\
\end{array}$ & $\begin{array}{c}\mathrm{Ga} \\
\mathrm{ppm}\end{array}$ & $\begin{array}{l}\mathrm{Ge} \\
\mathrm{ppm}\end{array}$ & $\begin{array}{l}\mathrm{Hf} \\
\mathrm{ppm}\end{array}$ & $\begin{array}{l}\mathrm{Hg} \\
\mathrm{ppm}\end{array}$ & $\begin{array}{c}\text { In } \\
\mathrm{ppm}\end{array}$ & $\begin{array}{l}\mathrm{K} \\
\% \\
\end{array}$ & $\begin{array}{c}\mathrm{La} \\
\mathrm{ppm}\end{array}$ & \begin{tabular}{|c|}
$\mathrm{Li}$ \\
$\mathrm{ppm}$
\end{tabular} & \begin{tabular}{|c|}
$\mathrm{Mg}$ \\
$\%$ \\
\end{tabular} & $\begin{array}{l}\mathrm{Mn} \\
\mathrm{ppm}\end{array}$ \\
\hline 690 & & & $<0.005$ & & & 8.2 & 70 & & & & & & & & & & & & & 23 & 0.09 & 0.044 & 1.22 & 31.4 & \begin{tabular}{|l|}
19.6 \\
\end{tabular} & \begin{tabular}{|l|}
0.82 \\
\end{tabular} & 612 \\
\hline & & & & & & & & & & & & & & & & & & & 0.19 & 2. & & & \begin{tabular}{|l|l}
1.46 \\
\end{tabular} & 31.2 & 22.7 & 1.12 & 01 \\
\hline & & & .005 & & & & 920 & & & & & 6.7 & & & 2.61 & 26.0 & & 1.60 & & 2. & & .042 & 1.34 & 2.5 & 1.2 & & 665 \\
\hline & & & & & & & & & & & & & & & & & & & & & & & & & & & 498 \\
\hline & & & 006 & 15 & 45 & 1.7 & 50 & .14 & 15 & 60 & 0.35 & 0.3 & 3. & & 2.89 & 23.9 & .65 & 5.05 & 0.21 & 2. & 15 & .043 & 138 & 30.5 & 21.8 & & 1015 \\
\hline & & & .005 & 0.15 & 82 & 5 & 20 & 86 & 13 & 14 & 0.23 & $\overline{6.4}$ & $\overline{08}$ & 122 & 2.62 & 18.0 & 2.74 & 3.55 & 0.19 & 2.2 & .29 & .042 & \begin{tabular}{|l|l}
1.14 \\
\end{tabular} & 28.3 & 19.8 & 0.79 & 356 \\
\hline & & & & & & & & & & & 0.23 & $\overline{01}$ & & & 1.9 & & 2.53 & & 0.17 & & & & & 24.9 & & & 406 \\
\hline & & & .005 & 10 & 55 & & 50 & 85 & $\overline{09}$ & 30 & & 53.9 & 17.4 & $\overline{34}$ & 2.23 & 17. & 2.75 & 2.95 & 0.21 & 2. & .21 & .040 & \begin{tabular}{|l}
1.02 \\
\end{tabular} & 27.3 & \begin{tabular}{|l|}
19.2 \\
\end{tabular} & & 03 \\
\hline & & & .005 & $\overline{12}$ & & & 60 & $\overline{98}$ & $\overline{12}$ & 51 & 0.29 & 63.4 & & & 2.50 & 22.6 & 3.06 & 13.65 & 0.23 & 2. & .15 & 0.046 & 1.16 & 31.7 & \begin{tabular}{|l|}
21.5 \\
\end{tabular} & 1.50 & 889 \\
\hline & & & 015 & 12 & & & 10 & $\overline{06}$ & 10 & 59 & \begin{tabular}{|l|l|}
0.25 \\
\end{tabular} & 76.1 & $\overline{8.8}$ & & 2.3 & & 3.18 & 13.45 & 0.24 & 2. & & 0.040 & \begin{tabular}{|l|}
1.10 \\
\end{tabular} & 38.6 & \begin{tabular}{|l|l|}
22.6 \\
\end{tabular} & & 889 \\
\hline & & & .005 & \begin{tabular}{|l|}
0.14 \\
\end{tabular} & 69 & & 60 & 20 & 11 & 57 & \begin{tabular}{|l|l|}
0.23 \\
\end{tabular} & 4.0 & 17. & 5 & 2.43 & 19 & 3.35 & 13.70 & 0.27 & 2. & .11 & 0.043 & 1.16 & 49.0 & 25.0 & 1.63 & 597 \\
\hline & & & 014 & 12 & & & & $\overline{02}$ & & 72 & \begin{tabular}{|l|l|}
0.28 \\
\end{tabular} & 1.6 & & 61 & $2.3 !$ & . & .40 & 13.55 & 0.22 & & .00 & 0.042 & \begin{tabular}{|l}
1.12 \\
\end{tabular} & 40.1 & \begin{tabular}{|l|}
22.2 \\
\end{tabular} & 2.0 & 703 \\
\hline & & & 005 & 53 & & & & & & & & & & & 15.55 & & 78 & 5.20 & 028 & & & .074 & & 57.4 & 39.9 & & 630 \\
\hline & & & 007 & 0.66 & .77 & 5 & $\overline{00}$ & 1.96 & 2.95 & .38 & \begin{tabular}{|l|l|}
0.44 \\
\end{tabular} & 34.0 & 15.2 & 95 & 18.80 & 97.7 & 4.93 & 18.55 & 0.30 & 1.3 & 0.04 & .100 & 2.03 & 69.6 & \begin{tabular}{|l|l|}
46.6 \\
\end{tabular} & 1.33 & 1165 \\
\hline & & & & & & & & & & & & & & & 3.36 & 35.5 & & 15.35 & 0.19 & & & .050 & & 28.1 & \begin{tabular}{|l|}
25.9 \\
\end{tabular} & & 479 \\
\hline & & & .005 & 0.17 & & & 30 & 34 & 22 & 46 & & & & & & 25. & & 5.55 & & 2 & & & & & 27.0 & & 43 \\
\hline & & & .005 & .22 & 40 & .0 & 10 & 1.58 & .48 & 65 & 0.27 & 5.3 & & & 5.0 & 25. & 3.50 & 5.35 & 0.22 & 2. & .19 & .060 & 1.30 & 44.6 & 27.7 & 1.0 & 885 \\
\hline & & & $\overline{00}$ & 14 & & & & & & & & & & & & & & 4.20 & & & & 052 & & & 25.9 & & 03 \\
\hline & & & .005 & 17 & 23 & $\perp$ & 90 & .26 & 18 & 1.62 & .25 & 5.9 & 13. & & 3.10 & 23. & 3.30 & 4.90 & 0.24 & 3. & .24 & .054 & 1.2 & 44.2 & 24.6 & 1. & 10 \\
\hline & & & 015 & & & & & & & & & & & & & 22. & & 2.95 & 0.2 & & & 14 & & & 21.4 & & 1085 \\
\hline & & & & 10 & & & & & & & & & & & 252 & & & 3.40 & & & & & & & & & 692 \\
\hline & & & 034 & 0.25 & 93 & & 70 & 104 & 0.14 & 1.36 & \begin{tabular}{|l|}
0.57 \\
\end{tabular} & 50.7 & 7 & & 78 & 34.4 & 2.75 & 11.55 & 0.17 & 1. & 0.25 & 37 & 0.96 & 25.9 & 21.6 & 0.97 & 1000 \\
\hline & & & 23 & 87 & & & & & & & \begin{tabular}{|l|}
1.98 \\
\end{tabular} & & & & & 37. & 3.05 & 4.90 & 0.20 & & & 55 & & 33.5 & 25.1 & & 619 \\
\hline & & & & & & & & & & & 0.60 & & & & & & & & & & & & & 37.0 & & & 569 \\
\hline & & & 018 & 67 & & & & 32 & 91 & 10 & & & & & & 42. & 3.60 & 15.30 & 0.2 & 2. & 2 & .177 & 1. & 33.9 & 27.2 & 1.0 & 1265 \\
\hline & & & & & & & & & & & & & & & & & & 0 & & & & 59 & & 7.8 & 5.6 & & 601 \\
\hline & & & & 48 & & & & & & & \begin{tabular}{|l|l|}
0.46 \\
\end{tabular} & 73.2 & & & & & 2.87 & & & 2 & & & & 35.5 & \begin{tabular}{|l|}
27.1 \\
\end{tabular} & & 522 \\
\hline & & & & & & & & & & & & & & & & & & & & & & 32 & & 34.0 & 28.7 & & 1660 \\
\hline & & & & & & & & & & & & & & & & & & & & & & & & & 26.2 & & 880 \\
\hline & & & & & & & & & & & & & & & & & & & & & & 5 & & & 24.6 & & 555 \\
\hline & & & & 28 & & & & & & & & & 11.6 & & & & 2.70 & 55 & 0.21 & & & 047 & & 6.5 & 26.1 & & 528 \\
\hline & & & & 78 & & & & & & & & & 7. & & & & & & & & & & & 20.8 & \begin{tabular}{|l|}
18.4 \\
\end{tabular} & & 308 \\
\hline & & & & & & & & & & & & & & & & & & & & & & & & & \begin{tabular}{|l|}
28.2 \\
\end{tabular} & & 964 \\
\hline & & & 023 & 0.92 & 30 & 6.0 & & & & 1. & \begin{tabular}{|l|}
1.34 \\
\end{tabular} & & & & & & 3.3 & 5.55 & & & & 120 & 1.28 & 33.3 & 26.1 & & .000 \\
\hline & & & & & & & & & & & & & & & & & & & & & & & & 2.5 & & & 250 \\
\hline & & & 34 & & & & & & & & & & & & & & & & & & & .065 & & & 29.0 & & 1915 \\
\hline & & & 010 & 0.21 & .34 & 64.0 & & 1.2 & & 1.62 & 0.28 & & 14 & 8 & & & 3.4 & 4.95 & 0.20 & 2. & 20 & .055 & 1.40 & 29.6 & 25.1 & 1.07 & 581 \\
\hline & & & & & & & & & & & & & & & & & & & & & & & & 210 & 24.4 & & \\
\hline & & & 26 & 0.22 & & & & & & & & & 12.8 & & & & & & & & & 068 & 1.32 & 35.9 & \begin{tabular}{|l|}
23.8 \\
\end{tabular} & & 587 \\
\hline & & & 084 & 0.71 & & 54. & 55 & & & 0. & & & 9 & 68 & & jל.0 & & 0.75 & 0.18 & 1. & 0.48 & 0.066 & 0.93 & 23.4 & \begin{tabular}{|l|}
17.4 \\
\end{tabular} & & 403 \\
\hline & & & & & & & & & & & & & & & & & & & & & & & & $\bar{L}$ & 26.4 & & 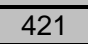 \\
\hline & & & 0.096 & 0.3 & 5.23 & $\overline{14.5}$ & 520 & 1.0 & 0.54 & 0.95 & 0.21 & 50.2 & 11. & 73 & 7.6 & 201.0 & 2.4 & & 0.2 & 1.7 & 0.64 & .063 & 1.08 & 24.7 & 23.5 & 0.86 & 424 \\
\hline & & & 0.005 & & & 12.4 & & & & & & & & & & & & & & & & & & 45.0 & 20.0 & & 02 \\
\hline
\end{tabular}


Table 1. Location and New Trace Element Geochemical Results for 902 Stream-Sediment Samples collected in the Livengood Area, Livengood Quadrangle, Alaska IS = Insufficient Sample for Analysis

\begin{tabular}{|c|c|c|c|c|c|c|c|c|c|c|c|c|c|c|c|c|c|c|c|c|c|c|c|c|c|c|}
\hline AMPLE & $\begin{array}{l}\text { Mo } \\
\text { ppm }\end{array}$ & $\begin{array}{c}\mathrm{Na} \\
\% \\
\end{array}$ & \begin{tabular}{|l|}
$\mathrm{Nb}$ \\
$\mathrm{ppm}$
\end{tabular} & $\begin{array}{r}\mathrm{Ni} \\
\mathrm{ppm}\end{array}$ & $\begin{array}{c}\mathrm{P} \\
\mathrm{ppm}\end{array}$ & $\begin{array}{c}\mathrm{Pb} \\
\mathrm{ppm}\end{array}$ & $\begin{array}{l}\mathrm{Rb} \\
\mathrm{ppm}\end{array}$ & $\begin{array}{c}\mathrm{Re} \\
\mathrm{ppm}\end{array}$ & $\begin{array}{l}\mathrm{S} \\
\%\end{array}$ & $\begin{array}{c}\text { Sb } \\
\mathrm{ppm}\end{array}$ & $\begin{array}{c}\text { Se } \\
\mathrm{ppm}\end{array}$ & $\begin{array}{l}S^{S n^{*}} \\
\text { ppm }\end{array}$ & $\begin{array}{c}\mathrm{Sr} \\
\mathrm{ppm}\end{array}$ & $\begin{array}{l}\mathrm{Ta}^{*} \\
\mathrm{ppm}\end{array}$ & $\begin{array}{c}\text { Te } \\
\text { ppm }\end{array}$ & $\begin{array}{c}\text { Th } \\
\text { ppm }\end{array}$ & $\begin{array}{l}\mathrm{Ti}^{\star} \\
\% \\
\end{array}$ & $\begin{array}{c}\mathrm{Tl} \\
\mathrm{ppm}\end{array}$ & $\begin{array}{c}\mathrm{U} \\
\mathrm{ppm}\end{array}$ & \begin{tabular}{|c|}
$\mathrm{V}$ \\
$\mathrm{ppm}$
\end{tabular} & $\begin{array}{l}W^{*} \\
\mathrm{ppm}\end{array}$ & \begin{tabular}{|c|}
$\mathrm{Y}$ \\
$\mathrm{ppm}$ \\
\end{tabular} & $\begin{array}{l}\mathrm{Zn} \\
\mathrm{ppm}\end{array}$ & $\begin{array}{l}\mathrm{Zr}^{\star} \\
\mathrm{ppm}\end{array}$ & $\begin{array}{c}\text { Pulp Wt } \\
\text { grams }\end{array}$ & $\begin{array}{c}\text { Lab } \\
\text { Report }\end{array}$ \\
\hline 1690 & 1.13 & 1.28 & 10.2 & 29.2 & 730 & 12.9 & 63.1 & $<0.002$ & 0.03 & 0.95 & 1 & 1.70 & 246.0 & 0.70 & $<0.05$ & 8.5 & 0.390 & 0.42 & 2.4 & 118 & 1.0 & 16.4 & 78 & 70.8 & 24 & FA04030732 \\
\hline 1692 & 0.74 & .34 & 10.4 & 35.1 & 910 & 12.7 & 65.1 & 0.002 & 0.05 & 1.14 & 1 & 1.70 & 246.0 & 0.75 & $<0.05$ & 8.7 & 0.390 & 0.41 & 2.4 & 124 & 1.0 & 17.9 & 91 & 68.1 & 45 & FA04030732 \\
\hline 1693 & 0.98 & 1.42 & 11.1 & 31.7 & 800 & 13.1 & 62.0 & $<0.002$ & 0.04 & 1.24 & 1 & 1.60 & 236.0 & 0.85 & \begin{tabular}{|l|}
0.05 \\
\end{tabular} & 9.1 & 0.417 & 0.38 & 2.3 & 124 & 1.1 & 18.0 & 80 & 72.2 & 7 & A04030732 \\
\hline 1694 & 0.84 & 40 & 0.9 & 30.5 & 820 & 11.8 & 59.7 & 0.002 & 0.04 & 1.12 & & 1.60 & 238.0 & \begin{tabular}{|l|l|}
0.78 \\
\end{tabular} & $<0.05$ & 8.8 & 0.421 & 0.36 & 2.3 & 120 & 12 & 17.3 & 78 & 70.5 & & A04030732 \\
\hline 1695 & 0.91 & 1.29 & 10.6 & 34.5 & 910 & 12.8 & 68.1 & $<0.002$ & 0.05 & 1.17 & 1 & 1.70 & 222.0 & 0.77 & $<0.05$ & 8.5 & 0.404 & 0.43 & 2.2 & 128 & 1.1 & 17.1 & 94 & 72.2 & 38 & FA04030732 \\
\hline 1696 & 0.69 & 1.11 & 9.0 & 46.8 & 660 & 11.2 & 58.0 & $<0.002$ & 0.03 & 0.94 & 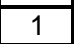 & 1.50 & 186.0 & 0.62 & $<0.05$ & 7.8 & 0.367 & 0.38 & 2.0 & 124 & 0.8 & \begin{tabular}{|l|l|}
14.4 \\
\end{tabular} & 72 & 67.1 & 23 & FA04030732 \\
\hline 1697 & 0.44 & 1.16 & 7.6 & 87.9 & 580 & 8.8 & 46.3 & $<0.002$ & 0.02 & 0.78 & 1 & 1.10 & 171.5 & 0.52 & $<0.05$ & 6.3 & 0.366 & 0.30 & 1.7 & 116 & 1.0 & \begin{tabular}{|l|}
13.6 \\
\end{tabular} & 68 & 57.7 & 25 & FA04030732 \\
\hline 1698 & 0.45 & 1.17 & 8.5 & 123.5 & 620 & 9.8 & 49.8 & $<0.002$ & 0.02 & 0.87 & & 1.30 & 181.5 & \begin{tabular}{|l|l|}
0.63 \\
\end{tabular} & $<0.05$ & 7.0 & 0.376 & 0.31 & 1.7 & 117 & $0 . \varepsilon$ & 14.3 & 78 & 60.9 & 24 & FA04030732 \\
\hline & 0.65 & .25 & 9.6 & 123.5 & 700 & 11.3 & 57.9 & $<0.002$ & 0.03 & 1.07 & & 1.50 & 198.0 & 0.68 & $<0.05$ & 8.6 & 0.405 & 0.37 & 2.0 & 124 & & 16.0 & $8 \varepsilon$ & 00.1 & & FA04030732 \\
\hline 1701 & 0.59 & 1.24 & 11.1 & 136.0 & 690 & 10.2 & 52.9 & $<0.002$ & 0.02 & 1.07 & 7 & 1.70 & 195.5 & 0.80 & $<0.05$ & 10.6 & 0.487 & 0.38 & 2.3 & 128 & 1.3 & 17.6 & 87 & 86.3 & $\overline{2}$ & FA04030732 \\
\hline 1702 & 0.76 & 1.21 & 12.6 & 103.0 & 830 & 9.8 & 53.3 & 0.002 & 0.02 & 1.34 & & 1.50 & 191.5 & \begin{tabular}{|l|}
0.91 \\
\end{tabular} & $<0.05$ & 11.7 & 0.533 & 0.34 & 2.6 & 137 & 1.1 & \begin{tabular}{|l|}
18.4 \\
\end{tabular} & 91 & 87.4 & 3 & FA04030732 \\
\hline & 0.62 & 1.28 & 11.3 & 156.5 & 740 & 10.1 & 52.6 & $<0.002$ & 0.02 & 1.11 & & 1.50 & 201.0 & 0.85 & $<0.05$ & 10.0 & 0.498 & 0.34 & 2.2 & 132 & 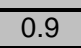 & \begin{tabular}{|l|}
17.8 \\
\end{tabular} & 90 & 85.4 & & \\
\hline 170 & 0.72 & 0.81 & 19.6 & 43.5 & 1920 & 32.5 & 124.0 & $<0.002$ & 0.05 & 2.17 & 7 & 6.20 & 352.0 & 1.50 & 0.06 & 14.8 & 0.407 & 0.87 & 5.4 & 130 & 1.9 & 20.8 & 141 & 23.1 & 30 & FA04030732 \\
\hline $170 !$ & 0.65 & 0.88 & 31.3 & 44.5 & 2310 & 33.5 & 151.0 & $<0.002$ & 0.02 & 2.65 & & 7.90 & 333.0 & 2.46 & 0.07 & 29.9 & 0.582 & 1.04 & 5.5 & 187 & 2.5 & 18.5 & 165 & 31.3 & 2 & FA04030732 \\
\hline 170 & 1.04 & 1.39 & 10.0 & 37.7 & 720 & 15.7 & 71.6 & $<0.002$ & 0.02 & 1.58 & & 1.90 & 224.0 & 0.70 & 0.05 & 8.6 & 0.349 & 0.46 & 2.5 & 118 & 1.0 & \begin{tabular}{|l|}
16.3 \\
\end{tabular} & 82 & 57.9 & & FA04030732 \\
\hline 170 & 0.62 & 1.43 & 10.8 & 33.7 & 690 & 14.2 & 70.7 & $<0.002$ & 0.03 & 1.37 & & 1.90 & 226.0 & 0.79 & $<0.05$ & 8.6 & 0.408 & 0.45 & 2.3 & 120 & 1. & 15.5 & 8 & 63.0 & 3 & FA04030732 \\
\hline 170 & 0.93 & 1.44 & \begin{tabular}{|l|}
16.6 \\
\end{tabular} & 31.1 & 1170 & 16.6 & 76.7 & 0.002 & 0.02 & 1.51 & & 2.40 & 247.0 & 1.19 & 0.06 & 14.2 & 0.453 & 0.51 & 4.3 & 124 & 1. & \begin{tabular}{|l|}
19.5 \\
\end{tabular} & 8 & 69.5 & & FA04030732 \\
\hline 171 & 0.77 & 1.43 & 10.9 & 32.3 & 730 & 12.6 & 63.2 & $<0.002$ & 0.02 & 1.37 & 1 & 1.80 & 227.0 & 0.74 & $<0.05$ & 9.8 & 0.431 & 0.45 & 2.2 & 118 & 1. & 17.2 & 7 & & & \\
\hline 171. & 0.88 & 1.37 & 13.2 & 34.0 & 850 & 13.5 & 64.0 & 0.002 & 0.03 & 1.40 & 1 & 1.90 & 229.0 & 0.98 & 0.05 & 12.1 & 0.497 & 0.40 & 2.9 & 126 & 1.4 & 19.3 & 80 & 83.3 & 5 & FA04030732 \\
\hline 177 & 0.53 & 1.20 & 9.0 & 162.0 & 580 & 10.4 & 55.2 & $<0.002$ & 0.04 & 1.18 & & 1.50 & 185.5 & 0.58 & $<0.05$ & 7.4 & 0.366 & 0.38 & 1.9 & 108 & 0.8 & 14.9 & 7 & 56.3 & & FA04030732 \\
\hline & 0.55 & 1.24 & 8.6 & 124.5 & 440 & 10.8 & 55.5 & $<0.002$ & 0.03 & 1.35 & & 1.60 & 174.5 & 0.63 & 0.05 & 6.8 & 0.373 & 0.35 & 1.8 & 11 & 0. & 14.3 & 6 & 56 & & 732 \\
\hline 1775 & 1.07 & 0.95 & 7.7 & 107.0 & 870 & 11.1 & 55.4 & $<0.002$ & 0.07 & 1.22 & & 1.50 & 158.0 & \begin{tabular}{|l|l|}
0.57 \\
\end{tabular} & 0.05 & 7.0 & 0.310 & 0.42 & 1.8 & 106 & 0.9 & 13.7 & 71 & 50.3 & 1 & FA04030732 \\
\hline 1776 & 2.12 & 1.21 & 9.8 & 31.9 & 740 & 21.7 & 68.8 & 0.002 & 0.03 & 1.52 & & 2.20 & 188.0 & 0.72 & 0.05 & 8.7 & 0.398 & 0.57 & 2.3 & 121 & 1.4 & 15.9 & 102 & 64.1 & & FA04030732 \\
\hline 1777 & 0.93 & 1.35 & 10.4 & 34.8 & 680 & 14.3 & 65.1 & $<0.002$ & 0.02 & 1.30 & & 2.00 & 214.0 & 0.75 & 0.05 & 10.5 & 0.432 & 0.46 & 2.4 & 123 & 1.0 & 16.9 & 88 & 64. & & 30732 \\
\hline 1778 & 2.71 & 1.10 & 10.0 & 38.3 & 800 & 38.7 & 71.2 & $<0.002$ & 0.03 & 2.32 & & 2.50 & 172.0 & 0.69 & 0.06 & 9.2 & 0.412 & 0.74 & 2.1 & 134 & 1.7 & \begin{tabular}{|l|}
15.8 \\
\end{tabular} & 138 & 63.0 & 4 & FA04030732 \\
\hline 17 & 0.87 & 1.22 & 9.7 & 29.1 & 630 & 16.2 & 66.0 & $<0.002$ & 0.03 & 1.09 & I & 2.10 & 182.5 & \begin{tabular}{|l|}
0.67 \\
\end{tabular} & $<0.05$ & 7.2 & 0.396 & 0.45 & 1.8 & 124 & 1. & \begin{tabular}{|l|l|}
12.4 \\
\end{tabular} & 81 & 56.5 & & FA04030732 \\
\hline 1780 & 0.85 & 1.18 & 10.6 & 31.5 & 580 & 21.2 & 68.1 & $<0.002$ & 0.02 & 1.01 & & 2.90 & 174.0 & 0.73 & 0.06 & 9.5 & 0.446 & 0.47 & 2.1 & 124 & 1.1 & 13.4 & 84 & 67. & & FA04030732 \\
\hline 178 & 1.53 & 1.18 & \begin{tabular}{|l|}
10.2 \\
\end{tabular} & 37.5 & 780 & 26.0 & 71.3 & $<0.002$ & 0.03 & 1.60 & & 2.30 & 185.5 & \begin{tabular}{|l|l|}
0.71 \\
\end{tabular} & 0.06 & 9.2 & 0.434 & 0.59 & 2.3 & 136 & 1.4 & 16.0 & 117 & 65.3 & & FA04030732 \\
\hline & & 1.26 & 11.2 & 38.7 & 730 & 22.3 & 67.3 & $<0.002$ & 0.1 & 1.6 & & 2.10 & 204.0 & 0.85 & 0.05 & 10.5 & 0.448 & 0.44 & 2.5 & 129 & 1. & 18.1 & 102 & 71 & & \\
\hline & 0.73 & 1.32 & \begin{tabular}{|l|l|}
10.8 \\
\end{tabular} & 33.7 & 630 & 16.2 & 58.7 & $<0.002$ & 0.02 & 1.31 & & 1.80 & 198.5 & 0.79 & $<0.05$ & 10.1 & 0.465 & 0.39 & 2.4 & 122 & 1. & \begin{tabular}{|l|}
16.1 \\
\end{tabular} & 90 & 63. & & FA04030732 \\
\hline 1784 & 0.61 & 1.34 & 10.3 & 32.6 & 630 & 14.2 & 58.1 & $<0.002$ & 0.02 & 1.29 & 1 & 1.80 & 205.0 & 0.73 & $<0.05$ & 9.6 & 0.430 & 0.35 & 2.1 & 111 & 1. & \begin{tabular}{|l|}
16.0 \\
\end{tabular} & 76 & 64.3 & & FA04030732 \\
\hline & 1.61 & 0.96 & 7.8 & 25.3 & 520 & 18.0 & 48.7 & & 0.04 & 1.11 & & 2.50 & 134.5 & 0.60 & 0.05 & 6.0 & 0.354 & 0.33 & 1.5 & 90 & 1. & \begin{tabular}{|l|}
9.5 \\
\end{tabular} & & 43 & & \\
\hline 178 & 2.22 & 1.09 & 9.7 & 33.4 & 730 & 33.9 & 72.7 & $<0.002$ & 0.04 & 1.88 & & 2.60 & 171.0 & 0.68 & 0.07 & 8.2 & 0.402 & 0.66 & 2.1 & 128 & 2.1 & \begin{tabular}{|l|}
13.6 \\
\end{tabular} & 123 & 58.5 & 37 & FA04030732 \\
\hline 1788 & 2.01 & 1.18 & 10.4 & 35.7 & 720 & 34.2 & 72.2 & $<0.002$ & 0.04 & 2.42 & & 2.40 & 182.5 & 0.73 & 0.07 & 8.8 & 0.416 & 0.60 & 2.3 & 129 & 1.7 & 16.4 & 122 & 64.6 & 2 & FA04030732 \\
\hline & 1.23 & 1.29 & 10.0 & 37.1 & 750 & 27.9 & 67.2 & & & 1.94 & & 2.10 & 197.0 & 0.72 & 0.0 & 8.9 & 0.415 & 0.49 & 2.1 & 126 & & \begin{tabular}{|l|}
15.4 \\
\end{tabular} & 114 & & & FA04030732 \\
\hline 1790 & 1.05 & 1.24 & 9.6 & 41.4 & 770 & 20.9 & 67.5 & $<0.002$ & 0.04 & 1.79 & 1 & 2.00 & 197.5 & 0.66 & 0.06 & 8.6 & 0.389 & 0.51 & 2.2 & 126 & 1.4 & 16.8 . & 150 & 62.2 & 3 & FA04030732 \\
\hline 1791 & 0.85 & 1.36 & \begin{tabular}{|l|}
10.2 \\
\end{tabular} & 41.2 & 900 & 12.2 & 73.3 & $<0.002$ & 0.06 & 1.39 & & 3.00 & 226.0 & \begin{tabular}{|l|}
0.68 \\
\end{tabular} & 0.08 & 8.5 & 0.385 & 0.61 & 2.1 & 122 & 1.9 & \begin{tabular}{|l|}
17.1 \\
\end{tabular} & 118 & 66.4 & 4 & A04030732 \\
\hline & 0.73 & 1.20 & 9.9 & 32.9 & 720 & 12.2 & 72.2 & & & 1.20 & & 5.80 & 172.5 & 0.66 & 0.06 & 7.7 & 0.434 & 0.48 & & & & 14.6 & 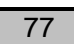 & 58.9 & & FA04030732 \\
\hline 1794 & 0.84 & 1.23 & 10.2 & 32.9 & 830 & 11.7 & 72.7 & $<0.002$ & 0.05 & 1.27 & 1 & 6.30 & 186.5 & 0.70 & 0.07 & 9.1 & 0.436 & 0.59 & 2.1 & 130 & 20.5 & \begin{tabular}{|l|}
15.1 \\
\end{tabular} & 63 & 64.9 & 30 & FA04030732 \\
\hline 1795 & 0.80 & 0.90 & 7.5 & 25.6 & 830 & 20.9 & 47.0 & 0.003 & 0.09 & 1.38 & & 3.20 & 129.0 & 0.53 & 0.05 & 6.1 & 0.316 & 0.39 & 2.1 & 87 & 2.4 & \begin{tabular}{|l|}
14.8 \\
\end{tabular} & 53 & 50.8 & 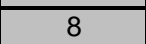 & A04030732 \\
\hline & 0.91 & $0.9 \varepsilon$ & 8.6 & 30.3 & 570 & 31.7 & 87.1 & $<0.002$ & 0.04 & 3.44 & 1 & 10.10 & 128.0 & 0.60 & & 7.5 & & 0.68 & 1.9 & 120 & 7.7 & & 6 & 5 & $\perp$ & FA04030732 \\
\hline 1797 & 0.78 & 0.94 & 7.7 & 35.2 & 790 & 13.3 & 63.9 & $<0.002$ & 0.08 & 1.39 & 2 & 3.30 & 145.0 & 0.55 & $<0.05$ & 6.4 & 0.317 & 0.45 & 1.7 & 102 & 0.9 & \begin{tabular}{|l|l|}
14.4 \\
\end{tabular} & 63 & 47.7 & 14 & FA04030732 \\
\hline 1798 & 1.10 & 1.44 & 12.8 & 36.3 & 800 & 15.8 & 63.8 & $<0.002$ & 0.03 & 1.52 & & 3.20 & 226.0 & 0.86 & 0.05 & 12.4 & 0.513 & 0.41 & 2.8 & 133 & 1.1 & 19.9 & 82 & 80.8 & 48 & FA04030732 \\
\hline
\end{tabular}


Table 1. Location and New Trace Element Geochemical Results for 902 Stream-Sediment Samples collected in the Livengood Area, Livengood Quadrangle, Alaska IS = Insufficient Sample for Analysis

\begin{tabular}{|c|c|c|c|c|c|c|c|c|c|c|c|c|c|c|c|c|c|c|c|c|c|c|c|c|c|c|c|}
\hline MPLE & TM E & TM N & $\begin{array}{c}\mathrm{Au} \\
\mathrm{ppm}\end{array}$ & $\begin{array}{c}\mathrm{Ag} \\
\mathrm{ppm}\end{array}$ & $\begin{array}{l}\text { Al } \\
\%\end{array}$ & $\begin{array}{l}\text { As } \\
\text { ppm }\end{array}$ & $\begin{array}{l}\mathrm{Ba}^{*} \\
\mathrm{ppm}\end{array}$ & \begin{tabular}{|c|}
$\mathrm{Be}$ \\
$\mathrm{ppm}$ \\
\end{tabular} & $\begin{array}{c}\mathrm{Bi} \\
\mathrm{ppm}\end{array}$ & $\begin{array}{c}\mathrm{Ca} \\
\% \\
\end{array}$ & \begin{tabular}{|c|}
$\mathrm{Cd}$ \\
$\mathrm{ppm}$ \\
\end{tabular} & $\begin{array}{c}\mathrm{Ce} \\
\mathrm{ppm}\end{array}$ & $\begin{array}{l}\text { Co } \\
\text { ppm }\end{array}$ & $\begin{array}{l}\mathrm{Cr}^{*} \\
\mathrm{ppm}\end{array}$ & $\begin{array}{c}\text { Cs } \\
\text { ppm }\end{array}$ & $\begin{array}{l}\mathrm{Cu} \\
\mathrm{ppm}\end{array}$ & $\begin{array}{c}\mathrm{Fe} \\
\% \\
\end{array}$ & $\begin{array}{c}\mathrm{Ga} \\
\mathrm{ppm} \\
\end{array}$ & $\begin{array}{c}\mathrm{Ge} \\
\mathrm{ppm}\end{array}$ & $\begin{array}{c}\mathrm{Hf} \\
\mathrm{ppm} \\
\end{array}$ & $\begin{array}{r}\mathrm{Hg} \\
\mathrm{ppm}\end{array}$ & $\begin{array}{c}\text { In } \\
\text { ppm }\end{array}$ & $\begin{array}{l}\mathrm{K} \\
\% \\
\end{array}$ & $\begin{array}{c}\mathrm{La} \\
\mathrm{ppm}\end{array}$ & $\begin{array}{c}\mathrm{Li} \\
\mathrm{ppm} \\
\end{array}$ & $\begin{array}{c}\mathrm{Mg} \\
\% \\
\end{array}$ & $\begin{array}{l}\mathrm{Mn} \\
\mathrm{ppm}\end{array}$ \\
\hline 799 & 8184 & 57601 & 0.098 & \begin{tabular}{|l|}
0.29 \\
\end{tabular} & \begin{tabular}{|l}
6.15 \\
\end{tabular} & 41.9 & 630 & $\begin{array}{l}1.12 \\
\end{array}$ & 2.88 & & \begin{tabular}{|l}
0.23 \\
\end{tabular} & & 17.3 & 116 & 4.63 & 154.0 & 3.21 & 13.80 & 0.20 & \begin{tabular}{|l|}
2.3 \\
\end{tabular} & 1.06 & 0.063 & \begin{tabular}{|l}
1.16 \\
\end{tabular} & 38.5 & 27.1 & 0.95 & 608 \\
\hline 800 & & & 19 & & \begin{tabular}{|l|l}
6.66 \\
\end{tabular} & & 760 & & 1.92 & & 0.35 & 76 & 19. & & 91 & 36 & 3.45 & 14.95 & 0.24 & 2.4 & 0.70 & 0.068 & \begin{tabular}{|l|}
1.30 \\
\end{tabular} & 37.6 & 1.8 & 1.02 & 54 \\
\hline & & & 0.015 & 26 & \begin{tabular}{|c|}
6.69 \\
\end{tabular} & 8.9 & 860 & 28 & 0.73 & 1.36 & 42 & 1.5 & 15.6 & & .1 & 0.2 & 3.81 & 15.75 & 0.27 & 2.5 & 0.41 & .060 & \begin{tabular}{|l|}
1.45 \\
\end{tabular} & 42.8 & 0.0 & 1.06 & 04 \\
\hline & & & 0.005 & 22 & \begin{tabular}{|l|l}
6.50 \\
\end{tabular} & 17.7 & 20 & 64 & & 1.49 & 40 & & 6.0 & & & & 3.36 & 4.65 & 0.20 & & & & 1.36 & 35.9 & 0.9 & 1.05 & \\
\hline 805 & & & 0.009 & .22 & 6.63 & 13.5 & 840 & 1.48 & 0.45 & 1.52 & \begin{tabular}{|l}
0.45 \\
\end{tabular} & 71.8 & 15.8 & 8 & 3.51 & 76.4 & 3.27 & 15.50 & 0.21 & 2.3 & 0.33 & 0.056 & 1.43 & 35.6 & 32.9 & 1.05 & 515 \\
\hline$\overline{06}$ & & & $\overline{009}$ & 24 & \begin{tabular}{|l}
6.17 \\
\end{tabular} & $\overline{1.3}$ & 760 & .10 & 0.98 & 1.30 & 0.36 & 66.2 & 16.8 & 86 & 3.80 & 88.9 & 3.32 & 14.10 & 0.22 & 2.0 & 0.28 & .053 & 1.29 & 32.8 & 25.2 & 0.99 & 15 \\
\hline & & & $\overline{005}$ & & 5.87 & & 790 & & & & 0.21 & & & & & & 2.99 & 13.20 & 0.19 & 23 & & & & 329 & & \begin{tabular}{|l}
0.97 \\
\end{tabular} & \\
\hline & & & .005 & 11 & 5.50 & 7.9 & 680 & 1.02 & 0.17 & 1.02 & 19 & 57.1 & 11.4 & 7 & 2.66 & 20.3 & 2.75 & 13.30 & 0.18 & 1.9 & 0.10 & .040 & 1.16 & 27.7 & 1.6 & 0.77 & 577 \\
\hline & & & 005 & 12 & 5.59 & $\overline{3.2}$ & 770 & 1.04 & 0.15 & 1.34 & 0.22 & 70.4 & 9.7 & & 2.51 & 18.4 & 2.71 & 13.85 & 0.19 & 2.2 & 0.09 & 0.041 & \begin{tabular}{|l|l|}
1.14 \\
\end{tabular} & 33.7 & 25.7 & 0.83 & 885 \\
\hline & & & $\overline{006}$ & 09 & 5.42 & 2.2 & 750 & 1.14 & 0.11 & 1.54 & \begin{tabular}{|l|l|}
0.24 \\
\end{tabular} & 77.2 & 9.8 & & 2.16 & 6.0 & 2.71 & 12.35 & 0.19 & 2.5 & 0.19 & 0.037 & \begin{tabular}{|l|}
1.10 \\
\end{tabular} & 38.0 & 24.7 & \begin{tabular}{|l}
0.87 \\
\end{tabular} & 85 \\
\hline$\overline{18}$ & & & 0.005 & 0.11 & 5.87 & 7.1 & 830 & 1.38 & 0.15 & 1.42 & \begin{tabular}{|l|}
0.31 \\
\end{tabular} & 64.2 & 9.9 & 7 & 2.46 & 21.1 & 2.82 & 13.00 & 0.17 & 2.1 & 0.11 & .041 & \begin{tabular}{|l|}
1.20 \\
\end{tabular} & 30.6 & 27.3 & \begin{tabular}{|l}
0.87 \\
\end{tabular} & 333 \\
\hline & & & 005 & 14 & 5.94 & .0 & 880 & 1.72 & & 1.66 & \begin{tabular}{|l|}
0.35 \\
\end{tabular} & 65.8 & 11. & & 2.6 & 0. & 2.95 & 13.70 & 0.18 & 2.2 & 0.13 & .046 & 1.28 & 31.8 & \begin{tabular}{|l|l|}
31.6 \\
\end{tabular} & 0.95 & 542 \\
\hline 18 & & & .005 & 08 & 78 & & 830 & 42 & & 1.67 & .23 & $\overline{5.9}$ & 9.8 & & $\overline{2.2}$ & 5. & 2.71 & 13.50 & 0.18 & 2.2 & $\sqrt{01}$ & .040 & \begin{tabular}{|l|l}
1.22 \\
\end{tabular} & & 5.9 & 0.92 & 67 \\
\hline & & & .005 & 0.10 & 5.85 & 7.4 & 810 & 1.20 & 0.12 & 1.64 & 0.23 & 74.6 & 10.6 & 8 & 2.30 & 17.0 & 2.80 & 13.25 & 0.19 & 2.4 & 0.07 & 0.041 & \begin{tabular}{|l|}
1.21 \\
\end{tabular} & 36.3 & 25.4 & 0.92 & 588 \\
\hline & & & 013 & & 5.20 & & & & & 1.04 & & & 7.4 & & & & 2.50 & 12.15 & 9 & $\overline{-1}$ & 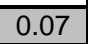 & 0.034 & \begin{tabular}{|l|}
1.05 \\
\end{tabular} & & 2.0 & 0.75 & \\
\hline & & & .005 & 12 & \begin{tabular}{|l|}
6.00 \\
\end{tabular} & 8.1 & 810 & & 0.1 & 1.42 & 26 & 67. & 12.2 & 8 & 2.68 & 19 & 2.99 & 4.45 & 0.19 & 2.2 & 0.0 & 442 & 1.28 & 32 & 8.8 & 0.94 & 999 \\
\hline & & & .005 & 10 & 5.83 & 8. & 800 & .38 & 0.15 & 1.44 & 0.28 & 69.6 & 13. & & 2.6 & 20.7 & 3.10 & 13.70 & 0.22 & 2.3 & 0.12 & .043 & 1.26 & 33.3 & 24.1 & 0.91 & 919 \\
\hline & & & 103 & 13 & 5.90 & & 8 & & & 36 & & & & & & & 2.8 & 3.90 & & & & 042 & 1.24 & & 5.1 & 0.86 & \\
\hline & & & .011 & 0.10 & 5.79 & 7.9 & 810 & 1.20 & 0.13 & 1.48 & 0.28 & 63.7 & 11. & 7 & 2.45 & 18. & 2.89 & 13.70 & 0.22 & 2.3 & 0.10 & $\overline{040}$ & 1.22 & 31.8 & 21.8 & 0.90 & 661 \\
\hline & & & 010 & & 5.97 & & 840 & & & 1.60 & $E_{0}$ & 13 & & & 2.5 & 19. & 2.91 & 13.95 & 0.21 & 2.6 & 0.1 & 042 & \begin{tabular}{|l|}
1.28 \\
\end{tabular} & 35.7 & 23.5 & 0.94 & 345 \\
\hline & & & .005 & 09 & \begin{tabular}{|l|l}
5.97 \\
\end{tabular} & 7.9 & 840 & 1 & & 1.68 & 32 & 77.8 & 12. & & 2.51 & 20. & 3.10 & 3.80 & 0.21 & & & 043 & 1.26 & & 11.5 & & 331 \\
\hline 184 & & & 0.005 & 0.10 & 5.83 & 8.8 & 830 & 1.14 & 0.13 & 1.47 & \begin{tabular}{|l|l}
0.32 \\
\end{tabular} & 68.4 & 12.4 & 8 & 2.58 & 22.0 & 3.07 & 13.85 & 0.20 & 2.4 & 0.12 & .039 & 1.26 & 32.5 & 21.2 & 0.93 & 714 \\
\hline$\overline{18}$ & & & $\overline{009}$ & 10 & 5.76 & & 600 & & & 2.17 & .27 & 56.4 & 28.4 & & 1.5 & 17. & 4.04 & 12.00 & 0.2 & 2.1 & 0.1 & .037 & 0.84 & 27.0 & 14.8 & 2.26 & 753 \\
\hline & & & $\overline{013}$ & 08 & 5.11 & 3 & 520 & & & 06 & & 49. & 32.5 & & 1.5 & & & & & 2.0 & & & & 23 & \begin{tabular}{|l|}
13.7 \\
\end{tabular} & & 51 \\
\hline & & & .005 & 11 & 5.40 & & 820 & 70 & & 1.32 & .24 & 64 & 18. & & 2.6 & 20. & 3.29 & 2.75 & 0.21 & 2. & 0.0 & .035 & 1.14 & 31.3 & 25.0 & 1.82 & 580 \\
\hline & & & & $\overline{09}$ & & 5 & & & & 2.11 & & & & & 1. & & 4.03 & 0.70 & 0.20 & & & 036 & 0.70 & 21.5 & 15.9 & 4.1 & 717 \\
\hline & & & & 11 & 5.17 & 5. & 510 & 0.68 & 0.06 & 2.09 & 0.21 & 57.8 & 35.0 & & 1.52 & & 4.03 & 1.15 & 0.21 & 2.1 & 0.07 & & \begin{tabular}{|l|}
0.75 \\
\end{tabular} & 27. & 7.0 & 3.8 & 37 \\
\hline & & & $\overline{010}$ & & 29 & 6 & 600 & & & 1.82 & & 58. & 29.5 & & 1.8 & 19 & 3.74 & 1.65 & $\overline{0.19}$ & 2. & 0.13 & .03 & 0.86 & 28.3 & \begin{tabular}{|l|}
18.9 \\
\end{tabular} & 3.24 & 666 \\
\hline & & & & & & & 570 & & & 2.1 & & & & & & & & .25 & & 2. & & & & & 9.8 & 3.5 & 18 \\
\hline & & & 005 & & & & 4 & & & & & & & & & & & 290 & & & & & & & & $\sqrt{47}$ & 367 \\
\hline 1857 & & & .005 & 0.12 & 5.32 & 6.3 & 500 & & & 1.64 & & 43.8 & 34.0 & & 1.66 & & 4.06 & 11.15 & 0.19 & 1.5 & 0.07 & .032 & 0.75 & 21.3 & 20.8 & 4.46 & 665 \\
\hline & & & 805 & & 5.14 & 5 & 500 & & & 1.72 & & 49 & & & & & 3.74 & & & 1.6 & 0.07 & & 0.78 & 24.0 & \begin{tabular}{|l|}
23.1 \\
\end{tabular} & $\begin{array}{l}4.02 \\
\end{array}$ & 520 \\
\hline & & & 0.008 & & & 6 & 560 & & & 83 & & & 25. & 68 & & $2 \varepsilon$ & 3.64 & 2.20 & & $\sqrt{21}$ & 0.09 & & 0.89 & 26.0 & 5.4 & 3.43 & 663 \\
\hline 18 & & & 0.006 & 0.18 & 5.76 & 8.2 & 880 & 1.65 & 0. & 1.34 & \begin{tabular}{|l|}
0.57 \\
\end{tabular} & 65.4 & 11 & 84 & 3.13 & 27.4 & 2.86 & 14.10 & 0.18 & 2.2 & 0.14 & .046 & 1.20 & 31.8 & 30.4 & \begin{tabular}{|l|} 
\\
\end{tabular} & 775 \\
\hline & & & & & & 9.1 & & & & & & & & & & & & & & 2.0 & & & 0.99 & 25.6 & 3.5 & 0.61 & 847 \\
\hline & & & .005 & & 5. & 10.9 & 80 & & & 1 & & & & & & & 2.81 & .40 & & 2.0 & 0.2 & .040 & 1.02 & 27.2 & 25. & 0.64 & 1335 \\
\hline & & 272 & 0.007 & 0.22 & 5.22 & 9.3 & 230 & 1.56 & 0.1 & 1.37 & 0.60 & 58. & 13.2 & & 3.0 & 37.1 & 2.63 & 12.65 & 0.19 & 2.2 & 0.35 & 044 & 1.08 & 28.3 & \begin{tabular}{|l|}
26.3 \\
\end{tabular} & 0.71 & 582 \\
\hline & & & & & & & & & & & & & & & & & & & & & & USt & & 33.2 & 0.9 & 0.71 & 1500 \\
\hline 1869 & & & 0.010 & 2 & 5.00 & & 1380 & 1. & 0. & 1.42 & & 57 & & 10 & 2.7 & 35.4 & 2.82 & 12.95 & 17 & 2.2 & 0.22 & 0.041 & 1.02 & 27.4 & 3.7 & \begin{tabular}{|l|}
0.80 \\
\end{tabular} & 836 \\
\hline 1870 & & & 0.013 & 0.22 & \begin{tabular}{|l|}
4.96 \\
\end{tabular} & 13.0 & 1310 & 1. & 0. & 1.29 & 0.78 & 56 & 16.6 & 7 & 2.7 & & 3.15 & 12.55 & 0 & 2.0 & 0.27 & .044 & \begin{tabular}{|l|} 
\\
\end{tabular} & 26.3 & \begin{tabular}{|l|}
22.2 \\
\end{tabular} & 0.75 & 1175 \\
\hline & & & & & & 10 & & & & 0.92 & & & & & & & 2.87 & & & 1.8 & 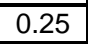 & & & 27.7 & 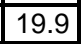 & 0.70 & 417 \\
\hline 187 & & 7271497 & $<0.005$ & 0.18 & 5.51 & 8.3 & 1060 & 1.22 & 0.1 & 1.41 & 0.38 & 57.8 & 14.4 & 7 & 2.5 & 26.4 & 2.65 & 15.00 & & 1.8 & 0.30 & .0 & 1.14 & 28.8 & 18.9 & 0.83 & 461 \\
\hline & & & 0.006 & 0.10 & & 7.6 & 1020 & & & & & 63.4 & 15.5 & 02 & 2.46 & & 2.70 & & & 1.9 & 0.31 & & 1.12 & 31.4 & & 0.84 & 551 \\
\hline
\end{tabular}


Table 1. Location and New Trace Element Geochemical Results for 902 Stream-Sediment Samples collected in the Livengood Area, Livengood Quadrangle, Alaska IS = Insufficient Sample for Analysis

\begin{tabular}{|c|c|c|c|c|c|c|c|c|c|c|c|c|c|c|c|c|c|c|c|c|c|c|c|c|c|c|}
\hline AMPLE & $\begin{array}{l}\text { Mo } \\
\text { ppm }\end{array}$ & $\begin{array}{c}\mathrm{Na} \\
\% \\
\end{array}$ & \begin{tabular}{|l|}
$\mathrm{Nb}$ \\
$\mathrm{ppm}$
\end{tabular} & $\begin{array}{r}\mathrm{Ni} \\
\mathrm{ppm}\end{array}$ & $\begin{array}{c}\mathrm{P} \\
\mathrm{ppm}\end{array}$ & $\begin{array}{c}\mathrm{Pb} \\
\mathrm{ppm}\end{array}$ & $\begin{array}{l}\mathrm{Rb} \\
\mathrm{ppm}\end{array}$ & $\begin{array}{l}\mathrm{Re} \\
\mathrm{ppm}\end{array}$ & $\begin{array}{l}\mathrm{S} \\
\%\end{array}$ & $\begin{array}{c}\text { Sb } \\
\mathrm{ppm}\end{array}$ & \begin{tabular}{|c|} 
Se \\
ppm
\end{tabular} & $\begin{array}{l}S^{S n^{*}} \\
\text { ppm }\end{array}$ & $\begin{array}{c}\mathrm{Sr} \\
\mathrm{ppm}\end{array}$ & $\begin{array}{l}\mathrm{Ta}^{*} \\
\mathrm{ppm}\end{array}$ & $\begin{array}{c}\text { Te } \\
\text { ppm }\end{array}$ & $\begin{array}{c}\text { Th } \\
\text { ppm }\end{array}$ & $\begin{array}{l}\mathrm{Ti}^{\star} \\
\% \\
\end{array}$ & \begin{tabular}{c|}
$\mathrm{Tl}$ \\
$\mathrm{ppm}$
\end{tabular} & $\begin{array}{c}\mathrm{U} \\
\mathrm{ppm}\end{array}$ & $\begin{array}{c}\mathrm{V} \\
\mathrm{ppm}\end{array}$ & $\begin{array}{l}W^{*} \\
\text { ppm }\end{array}$ & \begin{tabular}{|c|}
$\mathrm{Y}$ \\
$\mathrm{ppm}$ \\
\end{tabular} & $\begin{array}{l}\mathrm{Zn} \\
\mathrm{ppm}\end{array}$ & $\begin{array}{l}\mathrm{Zr}^{\star} \\
\mathrm{ppm}\end{array}$ & $\begin{array}{c}\text { Pulp Wt } \\
\text { grams }\end{array}$ & $\begin{array}{c}\text { Lab } \\
\text { Report }\end{array}$ \\
\hline 1799 & 0.93 & 1.20 & 10.4 & 36.7 & 670 & 18.4 & 63.7 & $<0.002$ & 0.03 & 1.62 & 1 & 4.00 & 176.0 & 0.70 & 0.05 & 9.7 & 0.449 & 0.48 & 2.2 & 122 & 76.4 & 17.1 & 84 & 64.8 & 27 & FA04030732 \\
\hline 1800 & 1.01 & 1.22 & 10.7 & 43.5 & 730 & 20.7 & 72.6 & $<0.002$ & 0.04 & 1.80 & 2 & 3.70 & 189.5 & \begin{tabular}{|l|l|}
0.74 \\
\end{tabular} & 0.05 & 9.5 & 0.423 & 0.53 & 2.3 & 128 & 6.1 & \begin{tabular}{|l|}
18.8 \\
\end{tabular} & 105 & 65.5 & 42 & FA04030732 \\
\hline 1802 & 2.36 & 1.26 & 11.7 & 39.8 & 850 & 25.3 & 78.7 & $<0.002$ & 0.04 & 2.00 & 2 & 2.10 & 204.0 & \begin{tabular}{|l|}
0.82 \\
\end{tabular} & 0.09 & 11.6 & 0.454 & 0.51 & 2.7 & 140 & 3.7 & 18.3 & 103 & 71.1 & 60 & A04030732 \\
\hline 1804 & 1.38 & 29 & 10.8 & 40.6 & 750 & 20.6 & 69.9 & $<0,002$ & 0.03 & 1.81 & & 2.60 & 205.0 & 0.75 & 0.06 & 10.1 & 0.432 & 0.48 & 2.5 & 127 & 13 & 17.9 & 98 & 66.3 & & A04030732 \\
\hline 1805 & 1.27 & 1.35 & 10.8 & 41.5 & 720 & 17.6 & 72.1 & $<0.002$ & 0.03 & 1.52 & 1 & 2.00 & 216.0 & 0.75 & 0.05 & 10.1 & 0.421 & 0.49 & 2.4 & 124 & 2.0 & 18.1 & 98 & 66.7 & 81 & FA04030732 \\
\hline 1806 & 1.53 & 1.19 & 9.9 & 38.7 & 700 & 19.9 & 65.6 & $<0.002$ & 0.03 & 1.62 & I & 2.40 & 184.0 & $\begin{array}{ll}0.67 \\
\end{array}$ & 0.07 & 8.1 & 0.414 & 0.40 & 2.1 & 122 & 4.2 & 16.1 & 100 & 65.6 & 3 & FA04030732 \\
\hline 1822 & 0.86 & 1.34 & \begin{tabular}{|l|}
10.4 \\
\end{tabular} & 26.4 & 700 & 11.3 & 52.2 & $<0.002$ & 0.02 & 1.12 & 1 & 1.50 & 219.0 & 0.71 & $<0.05$ & 9.0 & 0.439 & 0.32 & 2.2 & 121 & 1.0 & \begin{tabular}{|l|}
16.2 \\
\end{tabular} & 72 & 75.3 & & FA04030732 \\
\hline 1823 & 1.21 & 1.05 & 9.7 & 22.0 & 620 & 13.0 & 56.7 & $<0.002$ & 0.03 & 1.02 & & 1.70 & 165.0 & 0.72 & $<0.05$ & 7.8 & 0.377 & 0.38 & 2.2 & 115 & 0.9 & 12.6 & 63 & 69.5 & 2 & FA04030732 \\
\hline & 1.08 & .21 & 10.8 & 22.7 & 640 & 12.5 & 56.3 & $<0.002$ & 0.03 & 1.11 & & 1.80 & 194.0 & 0.75 & \begin{tabular}{|l|}
0.06 \\
\end{tabular} & 8.9 & 0.417 & 0.36 & 2.6 & 114 & $1 .$. & 15.3 & & 15.1 & & FA04030732 \\
\hline 1827 & 0.91 & 1.27 & 11.2 & 22.1 & 690 & 11.8 & 49.5 & $<0.002$ & 0.02 & 1.09 & . & 1.70 & 208.0 & 0.75 & $<0.05$ & 11.3 & 0.462 & 0.31 & 2.8 & 112 & 1.6 & 16.0 & 70 & 80.1 & & A04030732 \\
\hline 1828 & 1.07 & 1.26 & 10.0 & 23.7 & 690 & 12.6 & 53.0 & $<0.002$ & 0.03 & 1.10 & & 1.60 & 203.0 & 0.69 & $<0.05$ & 8.7 & 0.402 & 0.37 & 2.4 & 116 & 0.9 & 14.6 & 71 & 67.9 & & FA04030732 \\
\hline & 1.04 & 1.34 & 10.6 & 29.7 & 760 & 13.1 & 57.9 & 0.002 & 0.03 & 1.31 & & 1.70 & 223.0 & 0.71 & \begin{tabular}{|l|}
0.05 \\
\end{tabular} & 9.3 & 0.401 & 0.39 & 2.5 & 115 & & 16.6 & 79 & 80.5 & & \\
\hline 183 & 0.73 & 1.40 & 10.5 & 24.8 & 730 & 11.0 & 52.7 & 0.002 & 0.02 & 1.04 & 7 & 1.60 & 226.0 & 0.70 & $<0.05$ & 8.9 & 0.411 & 0.32 & 2.2 & 113 & 0.9 & 15.6 & 74 & 72.1 & & FA04030732 \\
\hline 1833 & 0.86 & 1.39 & 11.0 & 25.5 & 760 & 11.1 & 53.8 & $<0.002$ & 0.02 & 1.07 & & 1.60 & 229.0 & 0.75 & $<0.05$ & 9.7 & 0.439 & 0.35 & 2.5 & 115 & 1.0 & 16.5 & 72 & 77.8 & 3 & FA04030732 \\
\hline 1835 & 0.97 & 1.11 & 8.7 & 18.7 & 540 & 11.4 & 47.4 & $<0.002$ & 0.03 & 0.92 & & 1.70 & 168.0 & 0.62 & $<0.05$ & 6.9 & 0.366 & 0.31 & 1.9 & 108 & 87 & \begin{tabular}{|l|l}
10.7 \\
\end{tabular} & 55 & 58.4 & & \\
\hline 183 & 1.18 & 1.30 & 11.0 & 26.5 & 710 & 13.4 & 56.7 & $<0.002$ & 0.02 & 1.24 & & 2.00 & 209.0 & 0.76 & 0.06 & 9.4 & 0.435 & 0.36 & 2.5 & 122 & 0. & 15.5 & 7 & 73.1 & & FA04030732 \\
\hline$\overline{1 \varepsilon}$ & 1.35 & 1.22 & 10.8 & 25.8 & 740 & 13.3 & 56.6 & $<0.002$ & 0.03 & 1.15 & & 1.70 & 201.0 & 0.76 & $<0.05$ & 8.8 & 0.435 & 0.38 & 2.6 & 122 & 0. & 15.8 & 79 & 82. & & A04030732 \\
\hline 1844 & 1.12 & 1.28 & 10.6 & 26.1 & 720 & 12.6 & 57.0 & $<0.002$ & 0.03 & 1.19 & 1 & 1.70 & 209.0 & 0.76 & $<0.05$ & 7.9 & 0.394 & 0.36 & 2.4 & 117 & 0. & 15.5 & 75 & 70 & & \\
\hline 1841 & 1.02 & 1.30 & 10.5 & 25.2 & 690 & 12.0 & 54.0 & $<0.002$ & 0.03 & 1.07 & 1 & 1.60 & 211.0 & 0.76 & $<0.05$ & 8.3 & 0.423 & 0.34 & 2.4 & 117 & 1.0 & 15.5 & 73 & 78.7 & 4 & FA04030732 \\
\hline 1844 & 0.96 & 1.36 & 11.4 & 28.5 & 720 & 11.6 & 56.9 & $<0.002$ & 0.03 & 1.11 & & 1.70 & 222.0 & 0.76 & $<0.05$ & 9.4 & 0.455 & 0.33 & 2.6 & 122 & 1.0 & 17.1 & 77 & 83.0 & & FA04030732 \\
\hline & 0.99 & 1.34 & 1.9 & 30.0 & 780 & 11.5 & 54.8 & $<0.002$ & 0.02 & 1.18 & & 1.70 & 225.0 & 0.86 & $<0.05$ & 10.0 & 0.488 & 0.32 & 2.6 & 126 & 1. & 18.1 & 78 & 85 & & 732 \\
\hline 184 & 1.11 & 1.23 & 10.6 & 30.2 & 730 & 12.0 & 56.2 & 0.002 & 0.02 & 1.15 & & 1.70 & 201.0 & \begin{tabular}{|l|l|}
0.74 \\
\end{tabular} & 0.06 & 8.9 & 0.432 & 0.34 & 2.4 & 121 & 1.3 & 18.5 & 79 & 77.1 & & FA04030732 \\
\hline 1847 & 0.26 & 1.38 & 8.7 & 204.0 & 490 & 9.0 & 36.3 & $<0.002$ & 0.03 & 0.94 & & 1.30 & 212.0 & 0.63 & $<0.05$ & 6.9 & 0.441 & 0.23 & 1.8 & 140 & 0.8 & 14.5 & 97 & 72. & & FA04030732 \\
\hline & 0.28 & 1.13 & 7.6 & 299.0 & 540 & 7.4 & 36.3 & $<0.002$ & 0.04 & 0.80 & 1 & 1.20 & 187.0 & \begin{tabular}{|l|}
0.51 \\
\end{tabular} & $<0.05$ & 6.3 & 0.407 & 0.23 & 1.5 & 124 & 0.6 & \begin{tabular}{|l|}
13.0 \\
\end{tabular} & 108 & 61. & & 0732 \\
\hline 1849 & 0.76 & 1.04 & 9.2 & 161.5 & 740 & 10.0 & 56.7 & $<0.002$ & 0.02 & 1.19 & & 1.60 & 165.0 & \begin{tabular}{|l|}
0.63 \\
\end{tabular} & $<0.05$ & 7.7 & 0.375 & 0.35 & 2.1 & 123 & 1. & \begin{tabular}{|l|}
14.2 \\
\end{tabular} & 88 & 64.6 & & 30732 \\
\hline 18 & 0.31 & 1.13 & 7.0 & 360.0 & 450 & 6.8 & 30.2 & $<0.002$ & 0.02 & 0.80 & 3 & 1.10 & 167.5 & \begin{tabular}{|l|}
0.53 \\
\end{tabular} & $<0.05$ & 5.2 & 0.414 & 0.19 & 1.4 & 136 & 1. & \begin{tabular}{|l|}
13.3 \\
\end{tabular} & 95 & $56 . \varepsilon$ & & A04030732 \\
\hline 1852 & 0.33 & 1.12 & 7.9 & 336.0 & 520 & 6.9 & 32.9 & $<0.002$ & 0.02 & 0.92 & & 1.10 & 166.0 & \begin{tabular}{|l|l|}
0.57 \\
\end{tabular} & $<0.05$ & 6.1 & 0.448 & 0.20 & 1.5 & 141 & 3. & 14.2 & 100 & 64.2 & & 30732 \\
\hline 1853 & 0.44 & 1.14 & 8.8 & 292.0 & 560 & 8.1 & 39.0 & 0.002 & 0.02 & 0.96 & & 1.20 & 171.0 & \begin{tabular}{|l|}
0.59 \\
\end{tabular} & $<0.05$ & 6.5 & 0.426 & 0.24 & 1.7 & 128 & 0. & 14.3 & 90 & 76.2 & & FA04030732 \\
\hline & & 1.15 & 9.2 & 271.0 & 590 & 7.5 & 38.2 & $<0.002$ & 0.01 & 0.95 & & 1.20 & 171.0 & 0.63 & $<0.05$ & 8.6 & 0.481 & 0.23 & 1.9 & 142 & & 17.1 & & 73 & & \\
\hline 1855 & 0.44 & 0.88 & 6.0 & 517.0 & 440 & 6.0 & 32.4 & 0.002 & 0.04 & 0.92 & & 1.00 & 125.5 & 0.42 & $<0.05$ & 4.7 & 0.320 & 0.19 & 1.2 & 114 & 0. & 11.1 & 8 & 53.4 & 2 & FA04030732 \\
\hline 1857 & 0.56 & 0.99 & 6.5 & 416.0 & 430 & 7.0 & 33.8 & $<0.002$ & 0.02 & 1.63 & 1 & 1.10 & 133.0 & 0.44 & $<0.05$ & 5.4 & 0.351 & 0.21 & 1.3 & 118 & 0. & \begin{tabular}{|l|l|}
11.4 \\
\end{tabular} & 8 & 56.6 & & FA04030732 \\
\hline & & 1.06 & 7.0 & 335.0 & 440 & 6.9 & 33.2 & 0.002 & 0.02 & 0.93 & & 1.10 & 142.5 & \begin{tabular}{|l|}
0.51 \\
\end{tabular} & $<0.05$ & 5.5 & 0.357 & 0.22 & 1.5 & 117 & 1. & \begin{tabular}{|l|}
11.6 \\
\end{tabular} & 77 & 53.8 & & \\
\hline 1859 & 0.74 & 1.14 & 8.4 & 292.0 & 530 & 8.0 & 39.0 & 0.002 & 0.03 & 1.50 & & 1.30 & 162.5 & 0.59 & $<0.05$ & 6.4 & 0.410 & 0.25 & 1.8 & 124 & 0.9 & 13.9 & 79 & 70.8 & 4 & FA04030732 \\
\hline 1861 & 1.30 & 0.94 & 10.0 & 30.3 & 990 & 14.7 & 61.9 & $<0.002$ & 0.05 & 2.67 & 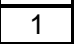 & 1.80 & 156.5 & 0.68 & $<0.05$ & 9.1 & 0.396 & 0.43 & 3.9 & 146 & 1. & 17.3 & 104 & 80.4 & 2 & FA04030732 \\
\hline & 1.63 & & 8.9 & 32.8 & & & 49.5 & & & 4.58 & & & 121.0 & \begin{tabular}{|l|}
0.61 \\
\end{tabular} & \begin{tabular}{|l|}
0.07 \\
\end{tabular} & 7.2 & & 0.56 & & 172 & & \begin{tabular}{|l|}
19.1 \\
\end{tabular} & 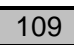 & 64 & & \\
\hline 1864 & 1.49 & 0.73 & 8.8 & 32.5 & 1260 & 12.8 & 51.5 & $<0.002$ & 0.05 & 4.11 & 2 & 1.60 & 142.0 & 0.64 & 0.06 & 7.9 & 0.345 & 0.67 & 4.7 & 192 & 0. & 18.9 & 114 & 72.4 & 2 & FA04030732 \\
\hline 1865 & 1.23 & 0.83 & 9.2 & 32.4 & 1290 & 12.6 & 52.8 & 0.002 & 0.05 & 4.89 & 2 & 1.60 & 160.5 & 0.66 & 0.06 & 8.3 & 0.379 & 0.59 & 4.7 & 176 & 0.8 & 18.0 & 106 & 71.0 & 2 & A04030732 \\
\hline & 2.02 & 0.87 & 10.4 & 32.6 & 880 & & 59.5 & $<0.002$ & 0.03 & 6.32 & & 2.20 & 154.0 & & 0.08 & & 0.406 & 0.52 & & 148 & & \begin{tabular}{|l|}
18.1 \\
\end{tabular} & 94 & 74.7 & & \\
\hline 1869 & 1.56 & 0.79 & 10.2 & 34.6 & 1400 & 12.4 & 53.0 & 0.002 & 0.05 & 7.72 & 2 & 1.60 & 156.5 & 0.70 & 0.07 & 7.3 & 0.431 & 0.50 & 4.1 & 174 & 0.9 & \begin{tabular}{|l|l|}
17.7 \\
\end{tabular} & 124 & 81.7 & 30 & FA04030732 \\
\hline 1870 & 1.57 & 0.69 & 9.4 & 34.9 & 1440 & 13.0 & 54.7 & 0.002 & 0.05 & 6.65 & & 1.90 & 136.5 & \begin{tabular}{|l|}
0.64 \\
\end{tabular} & 0.05 & 7.2 & 0.398 & 0.43 & 3.2 & 152 & 0.8 & 16.6 & 114 & 67.7 & 20 & A04030732 \\
\hline & 1.56 & $0.9 \varepsilon$ & 9.8 & 27.4 & 760 & 14.2 & 61.1 & $<0.002$ & 0.02 & 3.48 & s & 2.10 & 156.5 & \begin{tabular}{|l|l|}
0.68 \\
\end{tabular} & 0.0 & 7.5 & & 0.44 & 2.6 & 140 & 1 & 15.0 & 70 & 65. & & A04030732 \\
\hline 1872 & 0.99 & 1.08 & 10.4 & 34.1 & 970 & 12.4 & 59.4 & $<0.002$ & 0.03 & 4.42 & $<1$ & 1.60 & 183.0 & 0.70 & 0.05 & 7.2 & 0.428 & 0.42 & 2.5 & 134 & 1.1 & \begin{tabular}{|l|}
18.3 \\
\end{tabular} & 104 & 71.6 & 21 & FA04030732 \\
\hline 1873 & 0.85 & 1.11 & 10.3 & 32.2 & 960 & 11.9 & 59.2 & $<0.002$ & 0.03 & 3.30 & $<1$ & 1.50 & 188.5 & \begin{tabular}{|l|l|}
0.71 \\
\end{tabular} & 0.05 & 8.3 & 0.425 & 0.40 & 2.6 & 126 & 1.0 & 18.6 & 89 & 72.1 & 24 & FA04030732 \\
\hline
\end{tabular}


Table 1. Location and New Trace Element Geochemical Results for 902 Stream-Sediment Samples collected in the Livengood Area, Livengood Quadrangle, Alaska IS = Insufficient Sample for Analysis

\begin{tabular}{|c|c|c|c|c|c|c|c|c|c|c|c|c|c|c|c|c|c|c|c|c|c|c|c|c|c|c|c|}
\hline MPLE & ITM E & TM N & $\begin{array}{c}\mathrm{Au} \\
\mathrm{ppm}\end{array}$ & $\begin{array}{c}\mathrm{Ag} \\
\mathrm{ppm}\end{array}$ & $\begin{array}{l}\mathrm{Al} \\
\%\end{array}$ & $\begin{array}{l}\text { As } \\
\text { ppm }\end{array}$ & $\begin{array}{l}\mathrm{Ba}^{*} \\
\mathrm{ppm}\end{array}$ & \begin{tabular}{|c|}
$\mathrm{Be}$ \\
$\mathrm{ppm}$ \\
\end{tabular} & $\begin{array}{c}\begin{array}{c}\mathrm{Bi} \\
\mathrm{ppm}\end{array} \\
\end{array}$ & $\begin{array}{c}\mathrm{Ca} \\
\% \\
\end{array}$ & \begin{tabular}{|c|}
$\mathrm{Cd}$ \\
$\mathrm{ppm}$ \\
\end{tabular} & $\begin{array}{c}\mathrm{Ce} \\
\mathrm{ppm}\end{array}$ & $\begin{array}{c}\text { Co } \\
\text { ppm }\end{array}$ & $\begin{array}{l}\mathrm{Cr}^{\star} \\
\mathrm{ppm}\end{array}$ & $\begin{array}{l}\mathrm{Cs} \\
\mathrm{ppm}\end{array}$ & $\begin{array}{c}\mathrm{Cu} \\
\mathrm{ppm}\end{array}$ & $\begin{array}{c}\mathrm{Fe} \\
\% \\
\end{array}$ & $\begin{array}{c}\mathrm{Ga} \\
\mathrm{ppm}\end{array}$ & $\begin{array}{c}\mathrm{Ge} \\
\mathrm{ppm}\end{array}$ & \begin{tabular}{|c|}
$\mathrm{Hf}$ \\
$\mathrm{ppm}$
\end{tabular} & $\begin{array}{l}\mathrm{Hg} \\
\mathrm{ppm}\end{array}$ & $\begin{array}{c}\text { In } \\
\text { ppm }\end{array}$ & $\begin{array}{l}\mathrm{K} \\
\% \\
\end{array}$ & $\begin{array}{c}\mathrm{La} \\
\mathrm{ppm}\end{array}$ & \begin{tabular}{|c|}
$\mathrm{Li}$ \\
$\mathrm{ppm}$
\end{tabular} & $\begin{array}{c}\mathrm{Mg} \\
\%\end{array}$ & $\begin{array}{l}\mathrm{Mn} \\
\mathrm{ppm}\end{array}$ \\
\hline 1874 & 8465 & 71095 & 0.006 & 0.15 & \begin{tabular}{|l}
5.93 \\
\end{tabular} & 8.4 & 1020 & \begin{tabular}{|l}
1.19 \\
\end{tabular} & 0.14 & & 0.52 & & 154 & & & 24.1 & 2.82 & 14.75 & 0.18 & \begin{tabular}{|l|}
2.0 \\
\end{tabular} & 0.17 & 0.046 & 1.22 & 31.8 & 20.9 & & 870 \\
\hline 1875 & & & 005 & 32 & \begin{tabular}{|l}
5.90 \\
\end{tabular} & 8.3 & 870 & 1.14 & 0.14 & 40 & 0.32 & 66.5 & 12.2 & & 2.52 & 19.6 & 2.84 & 14.65 & 0.16 & 2.1 & 0.24 & 0.043 & 1.19 & 34.6 & 0.1 & 0.88 & 482 \\
\hline & & & D11 & 16 & 5.85 & 9.0 & 920 & & 0.14 & 54 & 31 & 63.1 & 13.4 & & 2.4 & 9.1 & 2.91 & 14.75 & 0.17 & 1.9 & 0.14 & 0.042 & 1.20 & 30.7 & 0.0 & 0.88 & 554 \\
\hline & & & & & \begin{tabular}{|l|l}
5.64 \\
\end{tabular} & 77 & 940 & & & & & & & & & & 2.47 & 1465 & 0 & & & & \begin{tabular}{|l|l}
1110 \\
\end{tabular} & & & 074 & 405 \\
\hline 1880 & 5129 & 69157 & 0.005 & 0.16 & 6.16 & 9.6 & 1000 & 1.30 & 0.15 & 1.36 & \begin{tabular}{|l|}
0.27 \\
\end{tabular} & 60.6 & 14.0 & 84 & 2.57 & 20.2 & 3.27 & 15.25 & 0.17 & 1.9 & 0.60 & 0.043 & 1.28 & 30.7 & 20.3 & 0.94 & 558 \\
\hline & & & D14 & 14 & 5.77 & 6.7 & 860 & .05 & 0.14 & 1.42 & 0.20 & 60.4 & 10.2 & 78 & 2.18 & 14.7 & 2.63 & 13.65 & 0.15 & 1.9 & 0.18 & 0.040 & 1.18 & 30.4 & 17.8 & 0.86 & 134 \\
\hline & & & 015 & $\overline{15}$ & \begin{tabular}{|l|l}
6.26 \\
\end{tabular} & 12.2 & 950 & 1.22 & $\overline{0.15}$ & 1.46 & \begin{tabular}{|l}
0.29 \\
\end{tabular} & 67.6 & 19.0 & 79 & 2.52 & 178 & 3.60 & 14.95 & 0.18 & 2.0 & 0.24 & 0.044 & 1.27 & 33.7 & \begin{tabular}{|l|l}
21.0 \\
\end{tabular} & \begin{tabular}{|l|}
0.94 \\
\end{tabular} & 99 \\
\hline & & & 0.013 & .14 & 4.30 & 35.1 & 560 & 0.81 & $\overline{0.11}$ & 8.88 & \begin{tabular}{|l|}
0.47 \\
\end{tabular} & 43.5 & 18.3 & 74 & 1.60 & 24.4 & 2.66 & 10.05 & 0.16 & 1.5 & 0.48 & .035 & 0.75 & 21.6 & 15.6 & 5.39 & 544 \\
\hline & & & & & \begin{tabular}{|l}
6.24 \\
\end{tabular} & & 850 & & & 2.09 & .36 & & 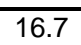 & & 6.49 & 6.4 & & 15.05 & .17 & $\bar{\Gamma}$ & 0.39 & & 1.23 & & 21.2 & 1.40 & $\overline{599}$ \\
\hline$\overline{1 \varepsilon}$ & & & .016 & 16 & 5.61 & 54.2 & 960 & .27 & 15 & 1.96 & .66 & 47.8 & 22. & & 2.78 & 24.6 & 4.38 & 14.10 & 0.19 & $\overline{1.6}$ & 0.28 & 0.040 & \begin{tabular}{|l}
1.17 \\
\end{tabular} & 24.5 & 2.0 & 0.99 & 2850 \\
\hline$\overline{18}$ & & & 0.005 & 0.15 & \begin{tabular}{|l|l}
6.20 \\
\end{tabular} & 8.9 & 860 & 1.14 & 0.14 & 1.66 & \begin{tabular}{|l|}
0.27 \\
\end{tabular} & 65.7 & 13.6 & 83 & 2.54 & 21.0 & 3.08 & 15.60 & 0.19 & 2.0 & 0.49 & 0.043 & 1.23 & 32.5 & 21.3 & 1.02 & 471 \\
\hline & & & & & \begin{tabular}{|l|l}
5.71 \\
\end{tabular} & & 780 & & & & & & & & 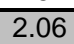 & & & 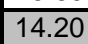 & & 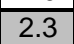 & 0.24 & & \begin{tabular}{|l|}
1.16 \\
\end{tabular} & 38.4 & & 1.16 & \\
\hline & & & 0.007 & 0.12 & \begin{tabular}{|l}
5.92 \\
\end{tabular} & 10.6 & 830 & & 0.10 & 2.19 & 25 & 61.5 & 12. & 85 & 2.1 & 16.6 & 3.10 & 13.55 & 0.18 & 1.9 & 0.42 & 0.040 & 1.20 & 31.8 & 18.6 & 1.22 & 595 \\
\hline & & & 0.016 & 0.13 & \begin{tabular}{|l|l}
6.16 \\
\end{tabular} & 12.0 & 840 & 1.15 & 0.12 & 2.04 & 0.25 & 80.1 & 13.8 & 100 & 2.28 & 17.1 & 3.29 & 14.80 & 0.19 & 2.4 & 0.18 & 0.041 & 1.20 & 40.4 & 39.9 & \begin{tabular}{|l|l}
1.12 \\
\end{tabular} & 661 \\
\hline & & & & 12 & \begin{tabular}{|l}
5.19 \\
\end{tabular} & 62 & 910 & & & 1.98 & \begin{tabular}{|l|}
0.32 \\
\end{tabular} & & 14.0 & & & & 2.80 & 12.60 & 0.18 & 1.8 & 8 & & \begin{tabular}{|l|l}
1.00 \\
\end{tabular} & & 57.1 & \begin{tabular}{|l|l|}
0.94 \\
\end{tabular} & 44 \\
\hline & & & 0.008 & 13 & 4.50 & 6.8 & 750 & 0.91 & 0.1 & 2.94 & 0.30 & 56.6 & 11. & & 1.7 & 15. & 2.45 & 11.70 & 0.18 & 1. & 0.1 & 035 & 0.93 & 28. & 27.8 & 1.45 & 483 \\
\hline & & & 0.008 & & 5.03 & & 850 & .91 & & 85 & .54 & & 12. & & 1.9 & $1 \%$. & 2.67 & 12.15 & 0.17 & 1. & 0.1 & 035 & \begin{tabular}{|l|l|}
0.97 \\
\end{tabular} & 29 & 31.0 & \begin{tabular}{|l|l}
1.26 \\
\end{tabular} & 397 \\
\hline & & & 0.008 & 13 & 5.51 & 8.7 & 790 & & & 2.51 & 30 & $\overline{06}$ & 11. & & 2.6 & 21 & & 12.75 & 016 & $\sqrt{1}$ & & & & & & & 444 \\
\hline & & & 0.008 & 0.16 & 6.05 & 25.2 & 710 & 0.90 & 0.14 & 1.23 & \begin{tabular}{|l|}
0.27 \\
\end{tabular} & 48.5 & 30.2 & 326 & 4.26 & 20.9 & 3.64 & 15.50 & 0.20 & 1.6 & 0.14 & 0.044 & 0.96 & 24.5 & 33.8 & \begin{tabular}{|l|l}
2.37 \\
\end{tabular} & $\overline{921}$ \\
\hline$\overline{1 s}$ & & & 201 & 12 & 5.82 & 55.8 & 570 & 0 & & 1.38 & \begin{tabular}{|l|}
0.34 \\
\end{tabular} & 43 & 32.8 & & 9.8 & 29.6 & 3.89 & 13.65 & 0.16 & 1. & 0.15 & 0.041 & \begin{tabular}{|l|l}
0.87 \\
\end{tabular} & 21.8 & 33.4 & 2.28 & 902 \\
\hline & & & 0.072 & & 5.70 & 99.0 & 670 & 1 & & 1.40 & 21 & 63. & 25. & & 5.7 & 19. & 3.25 & 13.50 & 0. & 1. & & & 1.0 & 32 & 6.9 & 2.1 & 97 \\
\hline & & & 0.011 & .15 & \begin{tabular}{|l}
5.67 \\
\end{tabular} & 16.7 & 810 & $1.1 !$ & 0.1 & 1.60 & 29 & 691 & 17. & 33 & 2.70 & 23. & 3.27 & 14.10 & $0.1 \varepsilon$ & 1.5 & 0.28 & .041 & \begin{tabular}{|l|}
1.13 \\
\end{tabular} & 34.8 & 25.2 & 1.58 & 653 \\
\hline & & & $\overline{573}$ & & 5.86 & & & & & 58 & & & 20. & & 3.8 & 19 & 3.26 & 4.35 & 0.1 & & 0.08 & .042 & 1.15 & 34.8 & 25.4 & 1.76 & 686 \\
\hline & & & .008 & 13 & \begin{tabular}{|l|l}
5.81 \\
\end{tabular} & 47.1 & 780 & 1.08 & 0.12 & 2.00 & 0.29 & 59.2 & 17.8 & 8 & 3.77 & 21. & 3.04 & 3.85 & 0. & 1. & 0.10 & & \begin{tabular}{|l|}
1.17 \\
\end{tabular} & 29.7 & 7.0 & \begin{tabular}{|l|}
1.59 \\
\end{tabular} & 642 \\
\hline & & & 009 & & \begin{tabular}{|l}
5.87 \\
\end{tabular} & 6.4 & 700 & & & 83 & & 51 & 8. & & 4.3 & 14. & 2.31 & 6.00 & 0.1 & 1. & 0.2 & .040 & 1.28 & 26.9 & 25.5 & \begin{tabular}{|l|}
0.75 \\
\end{tabular} & 348 \\
\hline 19 & & & 0.021 & 0.32 & 6.26 & 7. & 760 & 1.33 & 0.1 & 0.75 & .14 & 57.7 & 12 . & & 6.00 & 22.2 & 2.73 & 17.25 & 0.17 & 2. & 0.29 & .047 & \begin{tabular}{|l|}
1.38 \\
\end{tabular} & 28.7 & 30.7 & 0.89 & 529 \\
\hline & & & $\overline{005}$ & $\begin{array}{l}0.18 \\
\end{array}$ & 6.14 & 5.4 & 730 & 1.10 & & 0.94 & 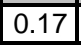 & 60.8 & 11. & & 378 & 201 & 2.72 & 6.45 & 0.18 & 1. & 0.28 & 046 & 1.24 & 31.4 & 30.3 & 0.93 & 456 \\
\hline 19 & & & .005 & 15 & 5.84 & 7. & 660 & & & 99 & .1 & 68. & 11. & & 3.2 & 16. & 2.71 & 5.90 & 0.1 & 2. & 0.16 & .046 & \begin{tabular}{|l|l} 
\\
\end{tabular} & 34.2 & 28.4 & \begin{tabular}{|l|l}
0.90 \\
\end{tabular} & 491 \\
\hline & & & 005 & & & & & & & 1.38 & & & & & & & & .05 & & 1. & & & 1.34 & 32.9 & 27.6 & \begin{tabular}{|l|l}
0.99 \\
\end{tabular} & 19 \\
\hline & & & 011 & & \begin{tabular}{|l}
6.43 \\
\end{tabular} & & 820 & 1.2 & & 1.28 & 228 & 69 & 14 & & 2.8 & 26 & 3.2 & 16.40 & 02 & 1. & 0.2 & .04 & 1.32 & 34.8 & 27.1 & \begin{tabular}{|l|}
0.98 \\
\end{tabular} & 533 \\
\hline 1947 & & & .005 & 0.10 & 5.85 & 6.5 & 710 & 1.11 & & 1.41 & 0.20 & 75.5 & & & 2.1 & 14. & 2.82 & 4.30 & 0.18 & 1. & .16 & .039 & 1.20 & 37.0 & 22.8 & 0.95 & 445 \\
\hline & & & 005 & & 5.87 & 77 & 740 & & & 1.30 & & & & & & & 2.80 & & & 1. & 26 & & 1.22 & 33.2 & 4.7 & 0.93 & $\overline{51}$ \\
\hline 19 & & & 017 & & 5.99 & 6. & 720 & & & $\overline{41}$ & & 70 & 12 . & & 2.4 & 15. & 2.87 & 4.35 & & 1. & 0.21 & .042 & 1.24 & 39.6 & 22.8 & \begin{tabular}{|l|l}
0.97 \\
\end{tabular} & $\overline{488}$ \\
\hline & & & .005 & & 5.90 & 6 & 670 & & & 1.40 & & 80.7 & 11. & & 2.2 & 12.8 & 2.79 & 13.80 & & 2. & 0.13 & .039 & \begin{tabular}{|l|l}
1.22 \\
\end{tabular} & 39.7 & 22.9 & 0.97 & 488 \\
\hline & & & & & & & 650 & & & & & & & & & & & & & & & & & & 7.2 & 0.68 & 277 \\
\hline 19 & & & 005 & & 6. & 9. & 710 & & & 85 & & & & & 2.9 & & 2.83 & 16.10 & & 2. & 0.09 & 0.044 & 1.45 & 33.7 & 24.0 & \begin{tabular}{|l} 
\\
\end{tabular} & 592 \\
\hline & & & .005 & .12 & \begin{tabular}{|l|l}
6.67 \\
\end{tabular} & 7.9 & 730 & 1. & 0. & 0.91 & 0.12 & 67.1 & 12.2 & 7 & 2.71 & 4.1 & 3.03 & 5.90 & 0.16 & 2.2 & 0.10 & .042 & 1.56 & 33.4 & 26.2 & \begin{tabular}{|l|l|}
0.84 \\
\end{tabular} & 481 \\
\hline & & & & & & & 660 & & & & & & & & & & & & & & & & 1.36 & 34.5 & 27 & \begin{tabular}{|l|l}
0.86 \\
\end{tabular} & 476 \\
\hline 195 & 9952 & 7238 & .005 & 16 & 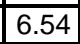 & 10.5 & 730 & 1. & 0. & 0.92 & \begin{tabular}{|l|l}
0.13 \\
\end{tabular} & 59.7 & 11. & & 3.3 & 16. & 3.15 & 16.70 & & 1.7 & 0.09 & 0.045 & 1.36 & 29.2 & 6.1 & 0.84 & 450 \\
\hline & & & .00 & 0.12 & \begin{tabular}{|l}
6.62 \\
\end{tabular} & 9.2 & 790 & & & 1.38 & & 6 & 12 & & 2.4 & & 3.05 & 15.90 & & 1.7 & 0.09 & .044 & 1.38 & 32.0 & 2.7 & 0.97 & 478 \\
\hline & & & & & & 8. & & & & & & & & & & & & & & & & & & 34.5 & & 0.85 & 379 \\
\hline 196 & & 2407 & 0.005 & 0.14 & 6.67 & 7.4 & 830 & 1.24 & 0.15 & 1.14 & 0.24 & 76.2 & 19.5 & 83 & 3.00 & 16. & 3.14 & 16.8 & & 2.4 & 0.19 & .0 & \begin{tabular}{|l}
1.57 \\
\end{tabular} & 38.7 & 36.3 & 0.91 & 1140 \\
\hline & & & & & 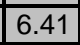 & 7.8 & 780 & & & & & 78.6 & 15.9 & & & 11.1 & 2.80 & & 0.10 & 2.8 & 0.17 & 0.044 & 1.58 & 39.6 & 30.1 & 0.86 & 673 \\
\hline
\end{tabular}


Table 1. Location and New Trace Element Geochemical Results for 902 Stream-Sediment Samples collected in the Livengood Area, Livengood Quadrangle, Alaska IS = Insufficient Sample for Analysis

\begin{tabular}{|c|c|c|c|c|c|c|c|c|c|c|c|c|c|c|c|c|c|c|c|c|c|c|c|c|c|c|}
\hline SAMPLE & $\begin{array}{l}\text { Mo } \\
\text { ppm }\end{array}$ & $\begin{array}{c}\mathrm{Na} \\
\%\end{array}$ & \begin{tabular}{|c|}
$\mathrm{Nb}$ \\
$\mathrm{ppm}$
\end{tabular} & $\begin{array}{c}\mathrm{Ni} \\
\mathrm{ppm}\end{array}$ & $\begin{array}{c}\mathrm{P} \\
\mathrm{ppm}\end{array}$ & $\begin{array}{l}\mathrm{Pb} \\
\mathrm{ppm}\end{array}$ & $\begin{array}{l}\mathrm{Rb} \\
\mathrm{ppm}\end{array}$ & $\begin{array}{l}\mathrm{Re} \\
\mathrm{ppm}\end{array}$ & $\begin{array}{l}\mathrm{S} \\
\%\end{array}$ & $\begin{array}{c}\mathrm{Sb} \\
\mathrm{ppm}\end{array}$ & \begin{tabular}{|c|} 
Se \\
ppm
\end{tabular} & $\begin{array}{l}\text { Sn* } \\
\text { ppm }\end{array}$ & $\begin{array}{c}\mathrm{Sr} \\
\mathrm{ppm}\end{array}$ & $\begin{array}{l}\mathrm{Ta}^{\star} \\
\mathrm{ppm}\end{array}$ & $\begin{array}{c}\text { Te } \\
\text { ppm }\end{array}$ & \begin{tabular}{|c|} 
Th \\
ppm
\end{tabular} & $\begin{array}{l}\mathrm{Ti}^{\star} \\
\%\end{array}$ & $\begin{array}{c}\mathrm{TI} \\
\mathrm{ppm}\end{array}$ & \begin{tabular}{|c|} 
\\
ppm
\end{tabular} & $\begin{array}{c}\mathrm{V} \\
\mathrm{ppm}\end{array}$ & $\begin{array}{l}W^{*} \\
\text { ppm }\end{array}$ & $\begin{array}{c}\mathrm{Y} \\
\mathrm{ppm}\end{array}$ & $\begin{array}{c}\mathrm{Zn} \\
\mathrm{ppm}\end{array}$ & $\begin{array}{l}\mathrm{Zr}^{*} \\
\mathrm{ppm}\end{array}$ & $\begin{array}{c}\text { Pulp Wt } \\
\text { grams }\end{array}$ & $\begin{array}{c}\text { Lab } \\
\text { Report }\end{array}$ \\
\hline 1874 & 0.96 & 1.22 & 10.2 & 33.5 & 950 & 12.0 & 64.1 & $<0.002$ & 0.03 & 2.86 & $<1$ & 1.80 & 201.0 & 0.70 & $<0.05$ & \begin{tabular}{|l|}
8.4 \\
\end{tabular} & 0.422 & 0.43 & 2.6 & 132 & 1.4 & 18.9 & 92 & 73.7 & 17 & FA04030732 \\
\hline 875 & 99 & 24 & 11.3 & 28.7 & 770 & 12.8 & 9.8 & $<0.002$ & 0.02 & 1.56 & $<1$ & 1.60 & 98.0 & \begin{tabular}{|l|}
0.81 \\
\end{tabular} & $<0.05$ & 9.2 & 0.453 & 0.41 & 2.5 & 121 & 1.4 & 16.9 & 75 & 75.5 & 32 & $A 04030732$ \\
\hline 1876 & 0.84 & .28 & 10.6 & 30.2 & 800 & 11.9 & 59.8 & 0.002 & 0.03 & 1.72 & $<1$ & 1.60 & 208.0 & 0.73 & $<0.05$ & 8.1 & 0.413 & 0.39 & 2.4 & 120 & 1.1 & 17.1 & 78 & 70.9 & 4. & FA04030732 \\
\hline 379 & 1.38 & 07 & \begin{tabular}{|l|}
10.8 \\
\end{tabular} & 24.2 & 760 & 11.8 & 60.5 & $<0.002$ & 0.03 & 1.07 & $<1$ & 1.70 & 168.5 & 0.75 & 0.05 & 7.3 & 0.425 & 0.41 & 2.6 & 130 & 1.1 & 14.5 & 61 & 66.6 & 13 & FA04030732 \\
\hline 880 & .14 & .22 & \begin{tabular}{|l|}
10.4 \\
\end{tabular} & 29.6 & 890 & 12.1 & 63.1 & $<0.002$ & 0.03 & 1.18 & $<1$ & 1.80 & 197.0 & \begin{tabular}{|l|}
0.71 \\
\end{tabular} & 0.05 & 8.1 & 0.427 & 0.42 & 2.3 & 130 & 1.1 & 16.9 & 79 & 72.1 & & FA04030732 \\
\hline 81 & 0.90 & 1.29 & 10.6 & 23.2 & 720 & 11.1 & 53.2 & $<0.002$ & 0.02 & 0.95 & $<1$ & 1.50 & 205.0 & \begin{tabular}{|l|}
0.73 \\
\end{tabular} & $<0.05$ & 8.0 & 0.439 & 0.35 & 2.0 & 118 & 1.2 & 16.1 & 63 & 68.3 & $2=$ & FA04030732 \\
\hline & & & 10.6 & 29.2 & 960 & & 64.2 & $<0002$ & 0.03 & 1.19 & $<1$ & & 215.0 & 0.72 & $<0.05$ & 8.7 & 0.414 & 0.40 & 2.3 & 123 & & & & & & \\
\hline 1885 & 0.50 & 0.66 & 8.4 & 36.1 & 1100 & 10.5 & 30.5 & $<0.002$ & 0.03 & 5.22 & $<1$ & 1.10 & 178.5 & 0.52 & $<0.05$ & 6.6 & 0.365 & 0.30 & 1.9 & 100 & 2.0 & 15.2 & 86 & 60.8 & 7 & FA04030732 \\
\hline 1886 & 0.47 & 1.38 & 11.0 & 41.5 & 1060 & 11.4 & 56.1 & $<0.002$ & 0.03 & 2.58 & $<1$ & 1.60 & 232.0 & 0.75 & $<0.05$ & 7.9 & 0.483 & 0.36 & 1.8 & 122 & 1.4 & 18.0 & 93 & 63.6 & 4 & FA04030732 \\
\hline & 0.96 & \begin{tabular}{|l|}
1.06 \\
\end{tabular} & 8.6 & 38.3 & 1280 & 12.5 & 66.8 & $<0.002$ & 0.09 & 13.00 & $<1$ & 1.70 & 182.5 & \begin{tabular}{|l|}
0.58 \\
\end{tabular} & 0.05 & 7.2 & 0.329 & 0.39 & 1.7 & 114 & 11 & 16.8 & 106 & 58.6 & & \\
\hline 88 & 0.56 & 1.30 & 11.9 & 31.7 & 760 & 12.0 & 61.6 & $<0.002$ & 0.03 & 2.42 & $<1$ & 1.70 & 207.0 & 0.80 & $<0.05$ & 8.7 & 0.531 & 0.45 & 2.2 & 120 & 1.1 & \begin{tabular}{|l|}
17.9 \\
\end{tabular} & 78 & 78.7 & & FA04030732 \\
\hline$\overline{1 \varepsilon}$ & 0.52 & 1.38 & 12.5 & 29.2 & 850 & 10.7 & 52.8 & $<0.002$ & 0.02 & 2.25 & $<1$ & 1.60 & 230.0 & 0.84 & $<0.05$ & 10.8 & 0.561 & 0.33 & 2.4 & 118 & 1.5 & 20.3 & 75 & 87.2 & 1 & FA04030732 \\
\hline & .53 & 1.40 & 11.0 & 29.8 & 830 & 10.3 & 53.3 & $<0.002$ & 0.02 & 2.33 & $<1$ & 1.40 & 231.0 & \begin{tabular}{|l|}
0.77 \\
\end{tabular} & $<0.05$ & 7.9 & 0.495 & 0.34 & 1.9 & 114 & & 17.5 & & 70.6 & & \\
\hline & 0.60 & 1.40 & 13.0 & 30.5 & 890 & 11.4 & 57.3 & $<0.002$ & 0.03 & 1.95 & $<1$ & 1.70 & 231.0 & 0.93 & $<0.05$ & 10.5 & 0.553 & 0.36 & 2.4 & 124 & 1.7 & 19.6 & 9 & 88.2 & & FA04030732 \\
\hline & 0.46 & 1.10 & 10.4 & 35.7 & 840 & 9.7 & 51.0 & $<0.002$ & 0.05 & 1.63 & $<1$ & 1.40 & 182.5 & \begin{tabular}{|l|}
0.68 \\
\end{tabular} & $<0.05$ & \begin{tabular}{|l|}
7.7 \\
\end{tabular} & 0.480 & 0.32 & 1.9 & 112 & $1.2=8$ & 15.8 & & 69.1 & & FA04030732 \\
\hline & 0.43 & $\overline{0.95}$ & 10.2 & 28.7 & 760 & 9.2 & 46.3 & $<0.002$ & 0.05 & 1.45 & $<1$ & 1.40 & 164.5 & 0.66 & $<0.05$ & 7.7 & 0.476 & 0.28 & 1.9 & & & 15.1 & 100 & 67 & & \\
\hline & 0.43 & 1.04 & 10.1 & 29.8 & 920 & 9.4 & 54.2 & $<0.002$ & 0.07 & 1.39 & $<1$ & 1.30 & 175.0 & 0.70 & $<0.05$ & 7.4 & 0.450 & 0.30 & 1.8 & 105 & 1.0 & 15.7 & 99 & 72.2 & 2 & FA04030732 \\
\hline & 44 & 1.22 & 9.2 & 31.1 & 800 & 11.3 & 53.3 & $<0.002$ & 0.04 & 1.97 & $<1$ & 1.40 & 198.0 & 0.63 & $<0.05$ & 6.7 & 0.375 & 0.34 & 1.7 & 103 & & 15.1 & & 60.1 & & 7732 \\
\hline & 0.91 & 1.16 & 8.9 & 211.0 & 550 & 11.2 & 51.3 & $<0.002$ & 0.03 & 3.13 & $<1$ & 1.50 & 159.5 & 0.62 & $<0.05$ & 6.5 & 0.396 & 0.38 & 1.6 & 130 & 1. & 13.5 & 97 & 59 & & FA04030732 \\
\hline 112 & 0.68 & 1.10 & 6.9 & 338.0 & 560 & 9.4 & 50.2 & $<0.002$ & 0.05 & 4.22 & $<1$ & 1.20 & 144.0 & \begin{tabular}{|l|}
0.47 \\
\end{tabular} & $<0.05$ & 5.6 & 0.358 & 0.54 & 1.6 & 130 & $18.8-2$ & 14.1 & 128 & 54.2 & & FA04030732 \\
\hline & 59 & 1.33 & 9.2 & 216.0 & 500 & 9.9 & 53.5 & $<0.002$ & 0.03 & 5.29 & & 1.3 & 199.0 & 0.64 & $<0.05$ & 8.3 & 0.403 & 0.41 & 2.0 & 114 & & 14.6 & 85 & 72 & & 732 \\
\hline & 0.78 & 1.22 & 10.2 & 104.0 & 780 & 12.2 & 57.1 & $<0.002$ & 0.02 & 3.85 & $<1$ & 1.50 & 203.0 & \begin{tabular}{|l|}
0.71 \\
\end{tabular} & $<0.05$ & 9.7 & 0.441 & 0.38 & 2.2 & 120 & 1.4 & 17.6 & 142 & 75.1 & & FA04030732 \\
\hline 15 & 0.71 & 1.34 & 10.3 & 137.5 & 700 & 10.8 & 55.7 & $<0.002$ & 0.02 & 3.32 & $<1$ & 1.50 & 209.0 & 0.71 & $<0.05$ & 9.3 & 0.440 & 0.36 & 2.0 & 122 & & 17.2 & 96 & 68.1 & & FA04030732 \\
\hline & 0.66 & 1.26 & 9.6 & 110.5 & 770 & 11.4 & 57.1 & $<0.002$ & 0.03 & 3.50 & $<$ & 1.50 & 202.0 & \begin{tabular}{|l|l|}
0.67 \\
\end{tabular} & $<0.05$ & 8.1 & 417 & 0.38 & 2.0 & & & 15.8 & & 67. & & 7732 \\
\hline 37 & 0.68 & 0.99 & 10.0 & 25.7 & 620 & 10.3 & 74.3 & $<0.002$ & 0.05 & 0.66 & $<1$ & 1.90 & 144.0 & \begin{tabular}{|l|l|}
0.67 \\
\end{tabular} & $<0.05$ & 6.4 & 0.380 & 0.46 & 1.7 & 113 & 00 & 12.6 & 55 & 62.4 & & FA04030732 \\
\hline 40 & 0.86 & 0.94 & 11.4 & 32.2 & 690 & 12.8 & 82.4 & $<0.002$ & 0.03 & 0.69 & $<1$ & 2.00 & 128.0 & \begin{tabular}{|l|}
0.76 \\
\end{tabular} & $<0.05$ & 7.3 & 0.438 & 0.51 & 1.9 & $13 \varepsilon$ & & 14.7 & 6 & 72.2 & & FA04030732 \\
\hline & 71 & 1.15 & 10.5 & 30.1 & 580 & 12 & 70.4 & $<0.002$ & 0.03 & 0.76 & $<$ & 1.80 & 151.0 & 0.70 & 0.05 & 8.1 & 0.447 & 0.46 & 1.9 & & & 15.4 & 7 & 67 & & \\
\hline & 0.78 & 1.18 & 10.9 & 29.1 & 520 & 11.2 & 65.4 & $<0.002$ & 0.02 & 0.73 & $<$ & 1.70 & 154.5 & 0.76 & $<0.05$ & 8.6 & 0.444 & 0.38 & 1.9 & 116 & & \begin{tabular}{|l|}
16.7 \\
\end{tabular} & & 72 . & & FA04030732 \\
\hline & 0.82 & 1.26 & 10.3 & 32.8 & 680 & 12.7 & 70.0 & $<0.002$ & 0.03 & 1.10 & & 1.90 & 191.5 & 0.69 & $<0.05$ & 9.5 & 411 & 0.44 & 1.9 & 1 & & 16.7 & 88 & 63 & & FA04030732 \\
\hline & & 1.28 & \begin{tabular}{|l|}
11.1 \\
\end{tabular} & 35.2 & 770 & 13.6 & 69.1 & $<0.002$ & 0.03 & 1.18 & $<1$ & 1.90 & 184.5 & \begin{tabular}{|l|}
0.79 \\
\end{tabular} & \begin{tabular}{|l|}
0.05 \\
\end{tabular} & 10.0 & 0.445 & 0.40 & 2.3 & 26 & & 18.6 & 107 & 69. & & FA04030732 \\
\hline 1947 & 0.60 & 1.40 & \begin{tabular}{|l|}
10.8 \\
\end{tabular} & 27.0 & 630 & 11.2 & 56.9 & $<0.002$ & 0.02 & 0.89 & $<1$ & 1.60 & 197.5 & 0.80 & $<0.05$ & 11.0 & 0.472 & 0.32 & 2.1 & 113 & 1.2 & 16.6 & 81 & 66.6 & 19 & FA04030732 \\
\hline & 0.67 & 1.32 & 10.2 & 29.9 & 620 & 11.3 & 62.5 & $<0.002$ & 0.02 & 0.93 & $<$ & 1.60 & 187.0 & \begin{tabular}{|l|}
0.76 \\
\end{tabular} & $<0.05$ & 9.0 & 0.411 & 0.36 & 1.9 & 108 & & 15.9 & 93 & 61. & $\mathrm{~g}+2+3$ & FA04030732 \\
\hline & 63 & 1.40 & 10.6 & 29.3 & 670 & & 59.2 & $<0002$ & 0.02 & 0.93 & & 1.70 & 198.0 & 0.76 & $<0.05$ & 10.4 & & 0.36 & & & & & 20 & 67. & & FA04030732 \\
\hline 50 & 0.56 & 1.42 & 10.8 & 28.0 & 640 & 10.1 & 55.9 & $<0.002$ & 0.01 & 0.84 & $<1$ & 1.60 & 197.5 & 0.82 & $<0.05$ & 10.2 & 0.489 & 0.33 & 2.2 & 114 & 1.2 & 17.4 & 124 & 70.2 & 27 & FA04030732 \\
\hline 51 & 0.51 & 1.06 & \begin{tabular}{|l|}
8.8 \\
\end{tabular} & 19.2 & 430 & 11.3 & 74.5 & $<0.002$ & 0.03 & 0.57 & $<1$ & 1.70 & 128.0 & 0.64 & $<0.05$ & \begin{tabular}{|l|}
9.6 \\
\end{tabular} & 0.368 & 0.43 & 2.0 & 74 & 0.9 & 13.0 & 77 & 74.5 & 1 & FA04030732 \\
\hline & & 1.04 & 9.0 & 24.2 & 510 & & 81.7 & $<0.002$ & 0.03 & 0.74 & $<1$ & 2.50 & 137.5 & \begin{tabular}{|l|}
0.67 \\
\end{tabular} & $<0.05$ & \begin{tabular}{|l|}
10.7 \\
\end{tabular} & 0.377 & 0.46 & 2.4 & 100 & & \begin{tabular}{|l|}
14.5 \\
\end{tabular} & 87 & 79.3 & & FA04030732 \\
\hline 1955 & 0.63 & 1.14 & 9.4 & 25.0 & 450 & 13.6 & 78.7 & $<0.002$ & 0.02 & 0.70 & $<1$ & 1.80 & 142.0 & \begin{tabular}{|l|}
0.75 \\
\end{tabular} & $<0.05$ & 13.5 & 0.384 & 0.46 & 2.2 & 98 & & \begin{tabular}{|l|l}
14.8 \\
\end{tabular} & 126 & 75.5 & & FA04030732 \\
\hline & 0.58 & 1.24 & 9.3 & 25.1 & 420 & 12.8 & 69.2 & $<0.002$ & 0.02 & 0.71 & $<1$ & 1.70 & 162.5 & \begin{tabular}{|l|}
0.74 \\
\end{tabular} & $<0.05$ & \begin{tabular}{|l|}
10.4 \\
\end{tabular} & 0.396 & 0.39 & 2.2 & 95 & & \begin{tabular}{|l|}
15.0 \\
\end{tabular} & 59 & 70.0 & & \\
\hline & 0.78 & 1.06 & 8.9 & 24.7 & 490 & & 78.3 & $<0.002$ & 0.03 & 0.90 & $<1$ & 1. & 143.0 & \begin{tabular}{|l|}
0.69 \\
\end{tabular} & $<0.05$ & 8.7 & 0.387 & 0.46 & 2.0 & 112 & & 12.3 & 68 & 56.8 & & FA04030732 \\
\hline 1960 & 0.68 & 1.38 & 9.2 & 29.9 & 560 & 13.5 & 70.6 & $<0.002$ & 0.02 & 1.03 & $<1$ & 1.70 & 200.0 & \begin{tabular}{|l|}
0.64 \\
\end{tabular} & $<0.05$ & 9.4 & 0.386 & 0.42 & 2.1 & 104 & 1.3 & \begin{tabular}{|l|}
16.1 \\
\end{tabular} & 76 & 60.9 & 48 & FA04030732 \\
\hline & & 0.98 & 9.9 & 33.8 & 710 & 18.5 & 90.8 & $<0.002$ & 0.05 & 0.79 & & 1.90 & 168.0 & 0.74 & $<0.05$ & 11.0 & 0.380 & 0.49 & 3.3 & 103 & & \begin{tabular}{|l|}
18.6 \\
\end{tabular} & 311 & 73.6 & E & \\
\hline 1966 & 0.62 & 1.10 & 11.0 & 35.5 & 700 & 16.3 & 83.8 & $<0.002$ & 0.04 & 0.69 & $<1$ & 1.90 & 183.5 & 0.80 & $<0.05$ & 10.9 & 0.412 & 0.49 & 3.1 & 100 & 1.1 & 18.4 & 136 & 81.2 & 31 & FA04C \\
\hline 1967 & 0.48 & 1.16 & \begin{tabular}{|l|}
11.2 \\
\end{tabular} & 30.8 & 550 & 12.9 & 78.8 & $<0.002$ & 0.02 & 0.59 & $<1$ & 1.60 & 167.0 & \begin{tabular}{|l|}
0.87 \\
\end{tabular} & $<0.05$ & \begin{tabular}{|l|}
11.9 \\
\end{tabular} & 0.424 & \begin{tabular}{|l|}
0.41 \\
\end{tabular} & \begin{tabular}{|l|}
2.6 \\
\end{tabular} & 88 & 1.0 & 16.1 & 130 & 96.1 & 24 & FA0403073 \\
\hline
\end{tabular}


Table 1. Location and New Trace Element Geochemical Results for 902 Stream-Sediment Samples collected in the Livengood Area, Livengood Quadrangle, Alaska IS = Insufficient Sample for Analysis

\begin{tabular}{|c|c|c|c|c|c|c|c|c|c|c|c|c|c|c|c|c|c|c|c|c|c|c|c|c|c|c|c|}
\hline MPLE & TM E & TM N & $\begin{array}{c}\mathrm{Au} \\
\mathrm{ppm}\end{array}$ & $\begin{array}{l}\mathrm{Ag} \\
\mathrm{ppm}\end{array}$ & $\begin{array}{l}\text { Al } \\
\%\end{array}$ & $\begin{array}{c}\text { As } \\
\text { ppm }\end{array}$ & $\begin{array}{l}\mathrm{Ba}^{*} \\
\mathrm{ppm}\end{array}$ & $\begin{array}{c}\mathrm{Be} \\
\mathrm{ppm} \\
\end{array}$ & $\begin{array}{c}\mathrm{Bi} \\
\mathrm{ppm}\end{array}$ & $\begin{array}{c}\mathrm{Ca} \\
\%\end{array}$ & $\begin{array}{c}\mathrm{Cd} \\
\mathrm{ppm}\end{array}$ & $\begin{array}{c}\mathrm{Ce} \\
\mathrm{ppm}\end{array}$ & $\begin{array}{l}\text { Co } \\
\text { ppm }\end{array}$ & $\begin{array}{l}\mathrm{Cr}^{*} \\
\mathrm{ppm}\end{array}$ & $\begin{array}{l}\text { Cs } \\
\text { ppm }\end{array}$ & $\begin{array}{l}\mathrm{Cu} \\
\mathrm{ppm}\end{array}$ & $\begin{array}{c}\mathrm{Fe} \\
\% \\
\end{array}$ & $\begin{array}{c}\mathrm{Ga} \\
\mathrm{ppm}\end{array}$ & $\begin{array}{l}\mathrm{Ge} \\
\mathrm{ppm}\end{array}$ & $\begin{array}{c}\mathrm{Hf} \\
\mathrm{ppm}\end{array}$ & $\begin{array}{l}\mathrm{Hg} \\
\mathrm{ppm}\end{array}$ & $\begin{array}{c}\text { In } \\
\text { ppm }\end{array}$ & $\begin{array}{l}\mathrm{K} \\
\% \\
\end{array}$ & $\begin{array}{r}\mathrm{La} \\
\mathrm{ppm}\end{array}$ & $\begin{array}{c}\mathrm{Li} \\
\mathrm{ppm} \\
\end{array}$ & $\begin{array}{c}\mathrm{Mg} \\
\% \\
\end{array}$ & $\begin{array}{l}\mathrm{Mn} \\
\mathrm{ppm}\end{array}$ \\
\hline 968 & 1467 & 40696 & $<0.005$ & \begin{tabular}{|l|l|}
0.14 \\
\end{tabular} & \begin{tabular}{|l}
6.17 \\
\end{tabular} & 8.5 & 750 & 1.08 & & & \begin{tabular}{|l|l|}
0.14 \\
\end{tabular} & & 105 & 77 & & 145 & 2.50 & 16.35 & 0.16 & & 0.16 & 0.044 & \begin{tabular}{|l}
1.58 \\
\end{tabular} & 32.9 & 23.4 & 0.71 & 385 \\
\hline & & & & & \begin{tabular}{|l}
7.03 \\
\end{tabular} & 31.0 & 900 & & & & & & $\overline{19}$ & & & 1.5 & 3.36 & 17.75 & 0.20 & 2.1 & 0.23 & 0.053 & \begin{tabular}{|l}
1.56 \\
\end{tabular} & 34.2 & 7.9 & \begin{tabular}{|l|}
0.91 \\
\end{tabular} & 1190 \\
\hline & & & .005 & 10 & 6.34 & 7.6 & 800 & 28 & 0.13 & 92 & 18 & 72.3 & 15.7 & & 2.63 & 3.0 & 2.84 & 16.15 & 0.16 & 2.8 & 0.13 & .042 & 1.58 & 35.8 & 9.4 & 0.79 & 1095 \\
\hline & & & & & \begin{tabular}{|l|l|}
6.54 \\
\end{tabular} & 7.5 & 810 & & & & & & & & & & & & 0.19 & & & & \begin{tabular}{|l|}
1.50 \\
\end{tabular} & 37.2 & 8.7 & \begin{tabular}{|l}
0.87 \\
\end{tabular} & \\
\hline $7 !$ & & & .005 & 13 & \begin{tabular}{|c|c|}
6.64 \\
\end{tabular} & 10.5 & 820 & .30 & .16 & 23 & .21 & 66.8 & 16. & 7 & 2.92 & 0.9 & 3.19 & 16.25 & 0.20 & 2.2 & 0.14 & 0.049 & \begin{tabular}{|l|l|}
1.50 \\
\end{tabular} & 33.5 & \begin{tabular}{|l|l}
31.2 \\
\end{tabular} & 0.93 & $\overline{903}$ \\
\hline & & & .005 & .09 & 6.41 & 11.2 & 760 & 18 & 0.13 & 1.30 & 0.18 & 69.0 & 13.8 & 78 & 2.37 & 14.5 & 2.87 & 15.45 & 0.20 & 2.0 & 0.10 & .041 & 1.46 & 34.4 & 27.4 & 0.92 & 742 \\
\hline & & & 005 & & 5.57 & 7.6 & 830 & & & 1.68 & \begin{tabular}{|l}
0.27 \\
\end{tabular} & 583 & & & 2.22 & 61 & 2.91 & & 0.18 & 1.9 & & & \begin{tabular}{|l|}
1.16 \\
\end{tabular} & 30.3 & & 0.94 & 529 \\
\hline & & & 0.005 & 0.15 & 5.90 & 11.0 & 960 & 1.18 & 0.14 & 1.92 & 0.36 & 62.2 & 17. & 8 & 2.46 & 24.9 & 3.95 & 15.10 & 0.20 & 2.2 & 0.14 & 0.049 & \begin{tabular}{|l|}
1.18 \\
\end{tabular} & 30.6 & 22.0 & 1.11 & 773 \\
\hline & & & 0.012 & 11 & 5.86 & 7.5 & 900 & 21 & 0.12 & 1.78 & 0.25 & 69.6 & 11.8 & & 2.24 & 15.7 & 2.89 & 13.80 & 0.18 & 2.2 & 0.06 & .039 & 1.26 & 35.1 & 21.8 & 0.95 & 526 \\
\hline & & & 0.005 & 14 & \begin{tabular}{|l|l}
6.32 \\
\end{tabular} & 10.3 & 1040 & .59 & 014 & 1.90 & \begin{tabular}{|l|l}
0.33 \\
\end{tabular} & 0.0 & 13 & & 2.69 & 22.9 & 3.17 & 14.85 & 0.20 & 1.9 & 019 & .045 & \begin{tabular}{|l|}
1.42 \\
\end{tabular} & 30.7 & 25.7 & 1.04 & 594 \\
\hline & & & $<0.005$ & .15 & 5.96 & 9.4 & 970 & 1.36 & 0.13 & 1.78 & 0.27 & 62.1 & 12. & 7 & 2.59 & 20.7 & 2.92 & 14.70 & 0.21 & 2.1 & 0.11 & 0.044 & \begin{tabular}{|l|}
1.30 \\
\end{tabular} & 31.2 & \begin{tabular}{|l|}
25.9 \\
\end{tabular} & 0.96 & 338 \\
\hline & & & .007 & 15 & \begin{tabular}{|l|l|}
6.33 \\
\end{tabular} & & 1000 & 36 & & 1.72 & \begin{tabular}{|l|}
0.31 \\
\end{tabular} & & I3. & & .63 & 19.6 & 2.95 & 14.65 & 0.19 & 1.8 & 0.18 & 0.043 & \begin{tabular}{|l|}
1.34 \\
\end{tabular} & 28.2 & 25.2 & 0.98 & 19 \\
\hline & & & .005 & 13 & & & 050 & 26 & & 75 & 35 & $\overline{5.4}$ & 13. & & 56 & $\overline{9}$ & 3.15 & 14.65 & 0.19 & 1.8 & 010 & .043 & 1.42 & 27.7 & 5.9 & 1.00 & 59 \\
\hline & & & 0.005 & 0.13 & 5.70 & 7.1 & 880 & 1.05 & 0.11 & 1.82 & 0.23 & 78.7 & 12. & 8 & 2.17 & 15.3 & 3.02 & 13.85 & 0.20 & 2.4 & 0.23 & 0.042 & 1.16 & 40.2 & 21.6 & 0.94 & 598 \\
\hline & & & .005 & & & & 870 & & & 1.69 & & & 12. & & 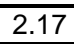 & . & 2.88 & 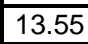 & 0.19 & 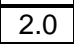 & 0.11 & 043 & 1.20 & 31.3 & 22.2 & 0.92 & 526 \\
\hline & & & 0.007 & 10 & 5.56 & 6.3 & 870 & 1 & 0.10 & 1.75 & 0.23 & 66.2 & & & 2.1 & 14. & 2.7 & 3.40 & 0.1 & 2.1 & 0.1 & 041 & \begin{tabular}{|l}
1.18 \\
\end{tabular} & 32.2 & 1.3 & 0.90 & 488 \\
\hline & & & 0.006 & 0.20 & \begin{tabular}{|l|}
5.97 \\
\end{tabular} & 9.4 & 910 & 1.15 & 0.15 & 1.44 & \begin{tabular}{|l|l}
0.46 \\
\end{tabular} & 54.0 & 17. & & 2.8 & 26. & 3.37 & 14.05 & 0.20 & 2.0 & 0.11 & .049 & \begin{tabular}{|l|}
1.17 \\
\end{tabular} & 26.9 & 26.2 & 1.00 & 779 \\
\hline & & & .00 & 14 & 83 & & 890 & & & 59 & & & & & & & & 3.85 & 0. & & & ים & 1.26 & 30.7 & 23.7 & 0.91 & \\
\hline & & & 0.005 & .11 & 6.23 & 8.2 & 1000 & 1.42 & 0.12 & 1.65 & .33 & 63.4 & 14. & 8 & 2.71 & 23.4 & 3.34 & 14.40 & 0.20 & 2.1 & 0.10 & $\overline{040}$ & 1.30 & 31.9 & 24.0 & 1.02 & $\overline{616}$ \\
\hline & & & .00 & & 5.83 & & 960 & & & 48 & 5 & & 14 & & 2.6 & 0. & 2.79 & 13.85 & 0.1 & 1. & 0.2 & 039 & 1.20 & 29.1 & 23.0 & 0.87 & 467 \\
\hline & & & .005 & 14 & & 9.3 & 950 & & & 80 & & & 15. & & & 17. & 3.20 & 3.90 & 0.20 & & 0.16 & 043 & 1.24 & & 3.3 & 0.96 & 325 \\
\hline & & & 0.005 & 0.15 & 6.00 & 9.7 & 970 & 1.43 & 0.14 & 1.74 & 0.33 & 57.9 & 13. & & 2.69 & 22.7 & 2.96 & 14.50 & 0.20 & 1.8 & 0.39 & .043 & 1.36 & 29.2 & 25.2 & 0.98 & 552 \\
\hline$\overline{19}$ & & & .005 & 16 & \begin{tabular}{|l}
6.02 \\
\end{tabular} & & 970 & & & 1.65 & 30 & 7. & 13. & & 2.6 & 8. & 3.18 & 4.70 & 0.26 & 1. & 0.3 & .040 & 1.30 & 30.6 & 25.2 & 0.95 & 642 \\
\hline & & & 008 & 12 & 5. & & 860 & & & 1.72 & & 80.6 & & & & & & & & 2. & & & 1.1 & 34.9 & \begin{tabular}{|l|l|}
21.4 \\
\end{tabular} & 0.98 & 44 \\
\hline & & & $<0.005$ & 0.09 & 5.33 & & 810 & & & 1.68 & 0.29 & & 13 & & 2.2 & & 2.9 & 11.95 & 0.2 & 2. & 0.18 & 0.044 & \begin{tabular}{|l|}
1.11 \\
\end{tabular} & 39.4 & 21.3 & 0.94 & 863 \\
\hline & & & .005 & 11 & & 9 & & & & 1.58 & & & 14 & & & & 3.00 & 2.65 & 0.2 & & 2 & 043 & $\overline{1.2}$ & 31.7 & 3.3 & $\overline{0.9}$ & 983 \\
\hline & & & .005 & 0.12 & 5.62 & 9. & 860 & 1.17 & & 1.42 & \begin{tabular}{|l|l}
0.29 \\
\end{tabular} & 63.9 & 16. & & 2.58 & 2 & 3.76 & 3.70 & 0.21 & 2.3 & 0.22 & .052 & \begin{tabular}{|l|}
0.96 \\
\end{tabular} & 29.2 & \begin{tabular}{|l|}
22.6 \\
\end{tabular} & 1.00 & 667 \\
\hline & & & 0.005 & & 79 & 7. & 860 & & & 1.80 & .29 & 68. & 15. & & 2.2 & 19. & 3.48 & 3.25 & 0.20 & 2.2 & 0.32 & .048 & \begin{tabular}{|l|}
1.09 \\
\end{tabular} & 29.4 & 21.4 & 1.13 & 610 \\
\hline & & & 005 & 12 & 5. & 8 & 930 & & & & & & & & & & & & & & & & & 31 & 3.1 & 0.6 & 18 \\
\hline & & & & & & & & & & & & & & & & & 80 & & & & & $\overline{0}$ & 1. & 37 & 2.1 & $\overline{06}$ & 530 \\
\hline 2010 & & & .005 & 13 & 5.62 & 8.5 & 900 & & & 1.62 & & $\overline{6}$ & 11. & & 2.2 & & 2.92 & 2.15 & 0.20 & 2. & 0.28 & .045 & 1.14 & 29.7 & 222.1 & 0.90 & 480 \\
\hline & & & & & & 7.4 & 860 & & & 1.88 & & 82 & & & & & 3.05 & & & 2.5 & & & 1. & 36.1 & 21.3 & 1.00 & $\overline{51}$ \\
\hline & & & $\overline{0}$ & & 5.52 & 8. & 850 & & & & & & & & & & 3.12 & 265 & & 2.5 & $\sqrt{21}$ & & & 38.0 & 2.1 & 0.98 & 689 \\
\hline & & & 0.006 & 0.09 & 5.78 & 9.2 & 890 & & 0. & 2.02 & \begin{tabular}{|l|l|}
0.30 \\
\end{tabular} & 146.0 & 15.4 & 37 & 2.3 & & 3.57 & 13.60 & 0.28 & 4.1 & 0.14 & .054 & 1.18 & 65.8 & 24.0 & 1.07 & 931 \\
\hline & & & & & \begin{tabular}{|l|l|}
5.49 \\
\end{tabular} & 12.4 & 940 & & & 1.60 & & & & & & & & & & 2.2 & 0.23 & & 1.18 & 29.6 & 5.8 & 0.87 & 1235 \\
\hline & & & 008 & 13 & & 9. & 760 & & & 10 & & & & & 2.5 & & 3.10 & 3.30 & & 2. & 0.17 & .046 & 1.09 & 32.2 & 2. & 0.83 & 919 \\
\hline 2016 & & 264 & 0.009 & 0.11 & 5.80 & 8. & 770 & 0. & 0. & 1.04 & 0.18 & 62. & 12. & & 2.6 & & 3.00 & 13.25 & 0.21 & 2.3 & 0.10 & .043 & \begin{tabular}{|l|l|}
1.10 \\
\end{tabular} & 27.3 & 24.2 & 0.82 & 523 \\
\hline & & & & & & & & & & & & & & & & & & & & & & & & & 3.4 & 080 & 472 \\
\hline 2019 & & 1 & $<0.005$ & 0.11 & 5.50 & 8.3 & 760 & & $\overline{0 .}$. & 1.00 & & & 17 & & 2. & 21.4 & 2.96 & 13.20 & 0.20 & 2.3 & 0.15 & .047 & 1.08 & 27.7 & 24.4 & 0.80 & 1095 \\
\hline & & & 0.008 & 0.11 & 5.59 & 8.7 & 780 & 1. & 0. & 1.30 & 0. & 7 & 13 & 7 & 2.4 & & 2.92 & 12.75 & 0 & 2.4 & 0.27 & .043 & \begin{tabular}{|l|l|}
1.12 \\
\end{tabular} & 31.1 & 22.8 & \begin{tabular}{|l}
0.87 \\
\end{tabular} & 704 \\
\hline & & & & & & 7. & 760 & & & & & & & & & & 2.8 & & & 2. & 0.14 & & & 37.6 & . & 0.84 & 24 \\
\hline 202 & & 726 & 0.018 & 0.12 & 5.45 & 8.8 & 750 & 1. & 0.1 & 1.25 & 0.22 & 81.2 & 14. & 75 & 2.4 & 19. & 2.92 & 12.90 & 0. & 2.6 & 0.18 & & 1.09 & 35.2 & 24.2 & 0.85 & 780 \\
\hline & & & $<0.005$ & \begin{tabular}{|l|}
0.10 \\
\end{tabular} & 10.0 & 8.8 & 790 & & & & & 11.9 & 14.3 & & 2.00 & & 3.02 & & & & 0.14 & & 1.15 & 33.8 & 23.8 & 0.89 & \\
\hline
\end{tabular}


Table 1. Location and New Trace Element Geochemical Results for 902 Stream-Sediment Samples collected in the Livengood Area, Livengood Quadrangle, Alaska IS = Insufficient Sample for Analysis

\begin{tabular}{|c|c|c|c|c|c|c|c|c|c|c|c|c|c|c|c|c|c|c|c|c|c|c|c|c|c|c|}
\hline AMPLE & $\begin{array}{l}\text { Mo } \\
\text { ppm }\end{array}$ & $\begin{array}{c}\mathrm{Na} \\
\% \\
\end{array}$ & \begin{tabular}{|l|}
$\mathrm{Nb}$ \\
$\mathrm{ppm}$
\end{tabular} & $\begin{array}{r}\mathrm{Ni} \\
\mathrm{ppm}\end{array}$ & $\begin{array}{c}\mathrm{P} \\
\mathrm{ppm}\end{array}$ & $\begin{array}{l}\mathrm{Pb} \\
\mathrm{ppm}\end{array}$ & $\begin{array}{l}\mathrm{Rb} \\
\mathrm{ppm}\end{array}$ & $\begin{array}{c}\mathrm{Re} \\
\mathrm{ppm}\end{array}$ & $\begin{array}{l}\mathrm{S} \\
\%\end{array}$ & $\begin{array}{c}\text { Sb } \\
\mathrm{ppm}\end{array}$ & $\begin{array}{c}\text { Se } \\
\mathrm{ppm}\end{array}$ & $\begin{array}{l}S^{S n^{*}} \\
\text { ppm }\end{array}$ & $\begin{array}{c}\mathrm{Sr} \\
\mathrm{ppm}\end{array}$ & $\begin{array}{l}\mathrm{Ta}^{*} \\
\mathrm{ppm}\end{array}$ & $\begin{array}{c}\mathrm{Te} \\
\mathrm{ppm}\end{array}$ & \begin{tabular}{c|} 
Th \\
ppm
\end{tabular} & $\begin{array}{l}\mathrm{Ti}^{\star} \\
\%\end{array}$ & $\begin{array}{c}\mathrm{Tl} \\
\mathrm{ppm}\end{array}$ & $\begin{array}{c}\mathrm{U} \\
\mathrm{ppm}\end{array}$ & $\begin{array}{c}\mathrm{V} \\
\mathrm{ppm}\end{array}$ & $\begin{array}{l}W^{*} \\
\text { ppm }\end{array}$ & \begin{tabular}{|c|}
$\mathrm{Y}$ \\
$\mathrm{ppm}$ \\
\end{tabular} & $\begin{array}{l}\mathrm{Zn} \\
\mathrm{ppm}\end{array}$ & $\begin{array}{l}\mathrm{Zr}^{\star} \\
\mathrm{ppm}\end{array}$ & $\begin{array}{c}\text { Pulp Wt } \\
\text { grams }\end{array}$ & $\begin{array}{c}\text { Lab } \\
\text { Report }\end{array}$ \\
\hline 1968 & 0.79 & 0.86 & 9.0 & 22.9 & 520 & 21.4 & 89.7 & $<0.002$ & 0.04 & 0.64 & $<1$ & 1.90 & 120.5 & 0.67 & $<0.05$ & 11.0 & 0.377 & 0.54 & 2.4 & \begin{tabular}{|l|}
91 \\
\end{tabular} & 1.0 & 14.7 & 90 & 73.7 & 13 & FA04030732 \\
\hline 1970 & 0.71 & .07 & 10.4 & 36.1 & 720 & 20.0 & 87.0 & $<0.002$ & 0.06 & 0.78 & $<1$ & 2.00 & 178.5 & 0.82 & $<0.05$ & 10.0 & 0.383 & 0.48 & 3.3 & 104 & 1.0 & 18.5 & 379 & 77.9 & 12 & A04030732 \\
\hline 1971 & 0.50 & 1.12 & 0.4 & 29.9 & 480 & 12.8 & 83.6 & $<0.002$ & 0.02 & 0.60 & $<1$ & 1.70 & 154.5 & 0.79 & $<0.05$ & 12.6 & 0.379 & 0.42 & 2.7 & 84 & 0.9 & 16.8 & 214 & 101.5 & & A04030732 \\
\hline & 0.55 & & 0.2 & 31.4 & 560 & 14.8 & 79.3 & $<0,002$ & 0.03 & 0.69 & $<1$ & 1.70 & 187.5 & 0.78 & $<0.05$ & 11.6 & 0.384 & 0.42 & 2.6 & 93 & 0.9 & 17.4 & 103 & 79.1 & 24 & $A 04030732$ \\
\hline 1975 & 0.58 & 1.18 & 9.7 & 32.4 & 640 & 15.9 & 78.7 & $<0.002$ & 0.04 & 0.87 & $<1$ & 1.70 & 189.5 & 0.76 & $<0.05$ & 10.3 & 0.381 & 0.45 & 2.7 & 102 & 1.0 & 17.4 & 143 & 75.1 & 21 & FA04030732 \\
\hline 1977 & 0.50 & 1.34 & 10.4 & 28.7 & 560 & 13.2 & 69.2 & $<0.002$ & 0.03 & 0.77 & $<1$ & 1.70 & 197.5 & 0.82 & $<0.05$ & 10.3 & 0.412 & 0.40 & 2.3 & 97 & 1.5 & \begin{tabular}{|l|}
16.6 \\
\end{tabular} & 130 & 69.8 & 21 & FA04030732 \\
\hline 1978 & 0.87 & 1.40 & 10.0 & 26.5 & 690 & 10.8 & 53.7 & $<0.002$ & 0.02 & 0.83 & $<1$ & 1.50 & 222.0 & 0.67 & $<0.05$ & 7.9 & 0.451 & 0.35 & 2.0 & 120 & 1.2 & \begin{tabular}{|l|}
17.3 \\
\end{tabular} & 71 & 70.2 & 14 & FA04030732 \\
\hline 198 & 1.14 & 1.34 & 11.1 & 32.6 & 850 & 12.4 & 56.3 & $<0.002$ & 0.03 & 1.03 & $<1$ & 1.70 & 218.0 & 0.74 & $<0.05$ & 7.8 & 0.579 & 0.36 & 2.2 & 156 & 1.1 & 20.8 & 92 & 77.6 & 3 & FA04030732 \\
\hline & 0.73 & .44 & $\overline{0.2}$ & 27.7 & 840 & 10.9 & 55.7 & $<0.002$ & 0.02 & 0.92 & $<1$ & 1.40 & 238.0 & 0.72 & $<0.05$ & 9.6 & 0.433 & 0.37 & 2.2 & 117 & 1.2 & 19.6 & & 73.6 & & FA04030732 \\
\hline 1982 & 0.88 & .46 & $\overline{10.1}$ & 34.1 & 860 & $\overline{12.4}$ & 63.2 & $<0.002$ & 0.03 & 1.15 & $<1$ & 1.50 & 245.0 & 0.71 & $<0.05$ & 8.2 & 0.404 & 0.40 & 2.0 & 122 & 1.0 & 18.1 & 86 & 71.9 & & FA04030732 \\
\hline 1983 & 0.82 & 1.43 & 10.2 & 31.8 & 810 & 12.6 & 62.7 & $<0.002$ & 0.02 & 1.22 & $<1$ & 1.60 & 237.0 & \begin{tabular}{|l|l|}
0.74 \\
\end{tabular} & $<0.05$ & 8.7 & 0.405 & 0.39 & 2.3 & 118 & 1.1 & 18.9 & 78 & 72.5 & 5 & FA04030732 \\
\hline & 0.79 & 1.45 & 9.5 & 32.0 & 790 & 12.1 & 64.4 & $<0.002$ & 0.03 & 1.04 & $<1$ & 1.50 & 235.0 & 0.67 & $<0.05$ & 7.6 & 0.371 & 0.38 & 2.0 & 113 & 1.3 & 17.9 & & 67.2 & & \\
\hline $198 !$ & 0.79 & 1.43 & 9.3 & 32.6 & 870 & 12.1 & 64.3 & $<0.002$ & 0.03 & 1.02 & $<1$ & 1.50 & 237.0 & 0.65 & $<0.05$ & 7.7 & 0.372 & 0.41 & 1.9 & 120 & 0.9 & 17.0 & 92 & 66.8 & & FA04030732 \\
\hline 198 & 0.65 & 1.35 & 11.4 & 27.3 & 880 & 10.6 & 52.7 & $<0.002$ & 0.02 & 0.82 & $<1$ & 1.50 & 223.0 & 0.82 & $<0.05$ & 10.4 & 0.539 & 0.33 & 2.4 & 122 & 1.4 & 19.8 & 76 & 82.9 & 7( & FA04030732 \\
\hline & 0.68 & 1.36 & 9.6 & 27.8 & 790 & 10.7 & 53.6 & $<0.002$ & 0.02 & 0.84 & $<1$ & 1.40 & 220.0 & 0.66 & $<0.05$ & \begin{tabular}{|l|}
8.0 \\
\end{tabular} & 0.435 & 0.34 & 1.9 & 116 & 1.5 & 17.7 & 76 & 69.7 & & \\
\hline 198 & 0.60 & 1.40 & 10.2 & 26.7 & 820 & 9.9 & 52.7 & $<0.002$ & 0.02 & 0.80 & $<1$ & 1.40 & 229.0 & 0.76 & $<0.05$ & 8.8 & 0.423 & 0.32 & 2.1 & 110 & 1.1 & 18.4 & 7 & 73.5 & 6 & FA04030732 \\
\hline 198 & 1.42 & 1.26 & \begin{tabular}{|l|}
9.4 \\
\end{tabular} & 35.3 & 780 & 12.7 & 58.1 & $<0.002$ & 0.03 & 1.13 & $<1$ & 1.60 & 190.5 & \begin{tabular}{|l|l|}
0.67 \\
\end{tabular} & $<0.05$ & 7.2 & 0.473 & 0.41 & 2.1 & 146 & 1.2. & 17.6 & 89 & 71.0 & & FA04030732 \\
\hline & 0.86 & 1.34 & 10.0 & 29.6 & 740 & 11.5 & 58.5 & $<0.002$ & 0.02 & 0.98 & $<1$ & 1.50 & 217.0 & 0.83 & $<0.05$ & 8.3 & 0.407 & 0.37 & 2.4 & 117 & 1.0 & 17.9 & 76 & 71.4 & & FA04030732 \\
\hline$\overline{1992}$ & 1.10 & 1.38 & 10.3 & 37.3 & 850 & 12.0 & 59.4 & $<0.002$ & 0.02 & 0.97 & $<1$ & 1.60 & 220.0 & 0.81 & $<0.05$ & 8.2 & 0.479 & 0.39 & 2.2 & 141 & 0.9 & 18.4 & 86 & 73.4 & $\overline{4 !}$ & FA04030732 \\
\hline 199 & 0.87 & 1.30 & 9.6 & 29.1 & 700 & 10.5 & 57.2 & $<0.002$ & 0.02 & 0.87 & $<1$ & 1.40 & 213.0 & \begin{tabular}{|l|l|}
0.67 \\
\end{tabular} & $<0.05$ & 7.2 & 0.390 & 0.37 & 2.0 & 121 & 1.1 & 16.8 & & 67.5 & & 30732 \\
\hline & 0.90 & .35 & 11.2 & 30.5 & 920 & 11.9 & 58.3 & $<0.002$ & 0.03 & 0.99 & $<1$ & 1.50 & 229.0 & 0.82 & $<0.05$ & 10.9 & 0.455 & 0.37 & 2.4 & 12 & 1. & 20.6 & & 83 & & 732 \\
\hline $199 !$ & 0.91 & 1.38 & 9.4 & 32.9 & 820 & 12.3 & 63.0 & $<0.002$ & 0.06 & 1.06 & $<1$ & 1.60 & 231.0 & 0.66 & $<0.05$ & 8.1 & 0.368 & 0.41 & 2.0 & 117 & 1.1 & 18.4 & 8 & 66.5 & & FA04030732 \\
\hline 1996 & 1.06 & 1.37 & 9.5 & 32.1 & 800 & 12.0 & 64.4 & $<0.002$ & 0.03 & 1.05 & $<$ & 1.60 & 224.0 & 0.69 & $<0.05$ & 7.8 & 0.379 & 0.40 & 2.0 & 118 & 1.2 & 17.5 & & 67.7 & & 30732 \\
\hline 1997 & 0.91 & 1.30 & 11.0 & 30.0 & 790 & 10.9 & 54.6 & $<0.002$ & 0.20 & 1.07 & $<1$ & 1.50 & 230.0 & 0.91 & $<0.05$ & 9.0 & 0.545 & 0.33 & 2.4 & 126 & 1.2 & 17.9 & 77 & 77.5 & & 30732 \\
\hline 1998 & 0.79 & 1.31 & 11.0 & 26.6 & 810 & 10.4 & 56.0 & $<0.002$ & 0.02 & 0.93 & $<1$ & 1.50 & 235.0 & 0.82 & $<0.05$ & 9.3 & 0.493 & 0.34 & 2.7 & 116 & 2.0 & \begin{tabular}{|l|}
18.7 \\
\end{tabular} & 7 & 80.6 & 5 & FA04030732 \\
\hline & 0.88 & 1.30 & 10.1 & 29.9 & 780 & 11.6 & 61.1 & $<0.002$ & 0.39 & 0.97 & $<1$ & 1.70 & 232.0 & 0.76 & $<0.05$ & 8.2 & 0.414 & 0.51 & 2.2 & 116 & 1. & 17.1 & & 70.2 & & 732 \\
\hline & 1.02 & 1.19 & 10.2 & 27.7 & 760 & 12.2 & 54.0 & $<0.002$ & 0.13 & 0.90 & $<1$ & 1.50 & 197.5 & 0.78 & $<0.05$ & 7.4 & 0.522 & 0.35 & 2.2 & 152 & 1. & 16.1 & 8 & 70.3 & & FA04030732 \\
\hline 200 & 0.87 & 1.42 & 10.5 & 29.5 & 760 & 11.3 & 52.2 & $<0.002$ & 0.02 & 0.90 & $<1$ & 1.50 & 241.0 & \begin{tabular}{|l|}
0.81 \\
\end{tabular} & $<0.05$ & 7.3 & 0.590 & 0.32 & 2.1 & 149 & 1. & 17.6 & 8 & 71.5 & & FA04030732 \\
\hline & & 1.34 & 10.0 & 30.4 & 810 & 12.4 & 59.1 & & 0.6 & 1.0 & $<1$ & 1.50 & 239.0 & 0.76 & $<0.05$ & 8.4 & 0.403 & 0.37 & 2.3 & 115 & 1 & 18.1 & & & & \\
\hline 200 & 0.82 & 1.40 & 11.0 & 28.4 & 800 & 11.2 & 55.5 & $<0.002$ & 0.02 & 0.95 & $<1$ & 1.50 & 251.0 & 0.83 & $<0.05$ & 9.5 & 0.523 & 0.34 & 2.4 & 124 & 1. & 19.0 & 7 & 77.3 & 6 & FA04030732 \\
\hline 2010 & 0.84 & 1.34 & 8.9 & 28.9 & 800 & 11.8 & 56.6 & $<0.002$ & 0.02 & 1.01 & $<1$ & 1.30 & 236.0 & 0.69 & $<0.05$ & 7.5 & 0.405 & 0.36 & 2.2 & 118 & 1. & \begin{tabular}{|l|}
16.4 \\
\end{tabular} & 7 & 61.2 & & FA04030732 \\
\hline & 0.71 & 1.46 & 11.2 & 28.0 & 850 & 10.7 & 52.7 & $<0.002$ & 0.02 & 0.89 & $<1$ & 1.40 & 260.0 & 0.86 & $<0.05$ & 9.3 & 0.560 & 0.32 & 2.3 & 126 & 0 & \begin{tabular}{|l|}
19.9 \\
\end{tabular} & 7 & 75.2 & & \\
\hline 201 & 0.82 & 1.41 & 11.0 & 29.5 & 840 & 11.6 & 53.8 & $<0.002$ & 0.02 & 0.94 & $<1$ & 1.50 & 251.0 & 0.82 & $<0.05$ & 9.8 & 0.533 & 0.33 & 2.6 & 126 & 1.2 & 19.2 & 76 & 77.7 & & FA04030732 \\
\hline 2013 & 0.93 & 1.38 & 16.2 & 31.9 & 1020 & 13.0 & 58.4 & $<0.002$ & 0.02 & 1.15 & $<1$ & 1.90 & 268.0 & 1.19 & $<0.05$ & 16.8 & 0.738 & 0.37 & 4.0 & 144 & 2. & \begin{tabular}{|l|}
26.8 \\
\end{tabular} & 82 & 22.5 & 5 & FA04030732 \\
\hline & & 1.19 & 9.3 & 33.2 & 810 & & 65.3 & & & 1.10 & & & 225.0 & \begin{tabular}{|l|l|}
0.68 \\
\end{tabular} & \begin{tabular}{|l|}
0.05 \\
\end{tabular} & 7.8 & 0.366 & 0.43 & 2.3 & 116 & & \begin{tabular}{|l|}
17.9 \\
\end{tabular} & & 66.7 & & FA04030732 \\
\hline 2015 & 1.25 & 1.23 & 10.4 & 24.4 & 740 & 13.0 & 56.7 & $<0.002$ & 0.03 & 0.90 & $<1$ & 1.50 & 201.0 & 0.75 & $<0.05$ & 8.4 & 0.466 & 0.36 & 2.4 & 120 & 1. & 16.6 & 7 & 80.5 & 3 & FA04030732 \\
\hline 2016 & 1.18 & 1.22 & 10.0 & 23.7 & 670 & 13.4 & 57.5 & $<0.002$ & 0.03 & 0.86 & $<1$ & 1.50 & 197.0 & 0.76 & $<0.05$ & 7.5 & 0.428 & 0.39 & 2.2 & 118 & 1.3 & \begin{tabular}{|l|}
14.5 \\
\end{tabular} & 69 & 74.0 & 3 & A04030732 \\
\hline & 1.11 & 1.22 & 9.9 & 23.7 & 620 & 13.0 & 56.8 & & 0.03 & 0.95 & $<1$ & 1.50 & 196.0 & 0.79 & $<0.05$ & 7.6 & 0.430 & 0.37 & 2.3 & 114 & & \begin{tabular}{|l|l|}
14.7 \\
\end{tabular} & 67 & 74.3 & & $=\mathrm{A} 04030732$ \\
\hline 2019 & 1.32 & 1.15 & 9.3 & 24.5 & 650 & 13.6 & 58.6 & $<0.002$ & 0.03 & 0.92 & $<1$ & 1.50 & 185.0 & \begin{tabular}{|l|l|}
0.67 \\
\end{tabular} & $<0.05$ & 7.4 & 0.396 & 0.39 & 2.2 & 115 & & \begin{tabular}{|l|l|}
14.7 \\
\end{tabular} & $7:$ & 68.0 & 28 & FA04030732 \\
\hline & 1.09 & 1.28 & 10.0 & 25.4 & 690 & 12.3 & 57.3 & $<0.002$ & 0.02 & 0.96 & $<1$ & 1.50 & 218.0 & 0.79 & \begin{tabular}{|l|}
0.05 \\
\end{tabular} & 8.0 & 0.438 & 0.32 & 2.3 & 114 & 1. & \begin{tabular}{|l|}
16.8 \\
\end{tabular} & 6 & 77.3 & 19 & $A 04030732$ \\
\hline & 1.06 & 1.30 & 106 & 25.8 & 640 & 12.0 & 58.4 & & 0.02 & 0.95 & $<1$ & 1.50 & 228.0 & 0.74 & $<0.05$ & 10.2 & & 0.36 & 2.5 & 114 & & & 0 & 16.4 & & FA04030732 \\
\hline 2022 & 1.17 & 1.24 & 10.6 & 26.4 & 700 & 12.1 & 58.4 & $<0.002$ & 0.03 & 0.98 & $<1$ & 1.60 & 210.0 & 0.77 & 0.06 & 8.9 & 0.458 & 0.37 & 2.5 & 113 & 1.2 & \begin{tabular}{|l|}
18.3 \\
\end{tabular} & 67 & 80.3 & 27 & 0732 \\
\hline 2024 & 1.22 & 1.25 & 10.4 & 27.7 & 730 & 13.6 & 59.4 & $<0.002$ & 0.03 & 1.00 & $<1$ & 1.60 & 214.0 & \begin{tabular}{|l|l|}
0.77 \\
\end{tabular} & 0.05 & 8.7 & 0.450 & 0.35 & 2.5 & 120 & 1.2 & 18.0 & 70 & 81.6 & 34 & FA04030732 \\
\hline
\end{tabular}


Table 1. Location and New Trace Element Geochemical Results for 902 Stream-Sediment Samples collected in the Livengood Area, Livengood Quadrangle, Alaska IS = Insufficient Sample for Analysis

\begin{tabular}{|c|c|c|c|c|c|c|c|c|c|c|c|c|c|c|c|c|c|c|c|c|c|c|c|c|c|c|c|}
\hline MPLE & TM E & TM N & $\begin{array}{l}\mathrm{Au} \\
\mathrm{ppm}\end{array}$ & $\begin{array}{l}\mathrm{Ag} \\
\mathrm{ppm}\end{array}$ & $\begin{array}{l}\text { Al } \\
\%\end{array}$ & $\begin{array}{c}\text { As } \\
\text { ppm }\end{array}$ & $\begin{array}{l}\mathrm{Ba}^{*} \\
\mathrm{ppm}\end{array}$ & \begin{tabular}{|c|}
$\mathrm{Be}$ \\
$\mathrm{ppm}$
\end{tabular} & $\begin{array}{c}\mathrm{Bi} \\
\mathrm{ppm}\end{array}$ & $\begin{array}{c}\mathrm{Ca} \\
\%\end{array}$ & \begin{tabular}{|c|}
$\mathrm{Cd}$ \\
$\mathrm{ppm}$
\end{tabular} & $\begin{array}{l}\text { Ce } \\
\text { ppm }\end{array}$ & $\begin{array}{l}\text { Co } \\
\text { ppm }\end{array}$ & $\begin{array}{l}\mathrm{Cr}^{\star} \\
\mathrm{ppm}\end{array}$ & $\begin{array}{l}\text { Cs } \\
\text { ppm }\end{array}$ & $\begin{array}{r}\mathrm{Cu} \\
\mathrm{ppm}\end{array}$ & $\begin{array}{c}\mathrm{Fe} \\
\% \\
\end{array}$ & $\begin{array}{c}\mathrm{Ga} \\
\mathrm{ppm}\end{array}$ & $\begin{array}{l}\mathrm{Ge} \\
\mathrm{ppm}\end{array}$ & $\begin{array}{r}\mathrm{Hf} \\
\mathrm{ppm}\end{array}$ & $\begin{array}{r}\mathrm{Hg} \\
\mathrm{ppm}\end{array}$ & $\begin{array}{c}\text { In } \\
\mathrm{ppm}\end{array}$ & $\begin{array}{l}\mathrm{K} \\
\% \\
\end{array}$ & $\begin{array}{c}\mathrm{La} \\
\mathrm{ppm}\end{array}$ & \begin{tabular}{|c|}
$\mathrm{Li}$ \\
$\mathrm{ppm}$
\end{tabular} & $\begin{array}{c}\mathrm{Mg} \\
\% \\
\end{array}$ & $\begin{array}{l}\mathrm{Mn} \\
\mathrm{ppm}\end{array}$ \\
\hline 2025 & 334 & 66131 & 0.007 & 0.14 & 5.48 & 8.2 & 740 & 1.11 & 0.16 & 1.02 & $\begin{array}{ll}0.27 \\
\end{array}$ & 59.6 & & 68 & 2.85 & 20.2 & 2.81 & 12.95 & 0.20 & 2.1 & 0.15 & 0.047 & 1.11 & 26.3 & \begin{tabular}{|l|}
25.0 \\
\end{tabular} & \begin{tabular}{|l|}
0.81 \\
\end{tabular} & 597 \\
\hline 026 & & & 007 & 0.09 & 68 & 10.2 & 870 & 1.29 & 0.15 & 30 & \begin{tabular}{|l|}
0.16 \\
\end{tabular} & 75.1 & 4. & 76 & .47 & 19.6 & 3.06 & 3.10 & 0.23 & 2.4 & 0.12 & .049 & \begin{tabular}{|l|l}
1.14 \\
\end{tabular} & 32.1 & 24.1 & \begin{tabular}{|l|l|}
0.89 \\
\end{tabular} & 648 \\
\hline 227 & & 66512 & 0.008 & .11 & 72 & 9.3 & 810 & 1.35 & 0.15 & 28 & \begin{tabular}{|l|l|}
0.33 \\
\end{tabular} & 76.4 & 19.3 & 76 & 2.57 & 20.2 & 3.16 & 12.85 & 0.23 & 2.4 & 0.29 & 0.047 & 1.15 & 31.8 & 24.0 & 0.89 & 1400 \\
\hline 228 & & & & 0.16 & & 8.4 & & 1.14 & & & & & & & & 22.0 & & 13.10 & 0.21 & 2.0 & 0.08 & & \begin{tabular}{|l}
1.12 \\
\end{tabular} & 25.9 & 24.9 & & 667 \\
\hline 029 & 1695 & 66500 & 0.013 & 0.11 & 5.46 & 8.9 & 760 & 1.21 & 0.16 & 1.15 & \begin{tabular}{|l|}
0.35 \\
\end{tabular} & 68.8 & 16.1 & 72 & 2.60 & 20.4 & 2.93 & 13.05 & 0.20 & 2.3 & 0.22 & 0.045 & 1.09 & 29.4 & 24.0 & 0.83 & 883 \\
\hline 2030 & & & 006 & 0.08 & 41 & 8.1 & 730 & 1.14 & 0.14 & 1.20 & 0.31 & 72.1 & 15.9 & 71 & 2.44 & 18.8 & 2.87 & 12.70 & 0.21 & 2.4 & 0.10 & 0.040 & 1.08 & 31.6 & 23.0 & 0.85 & 908 \\
\hline$\overline{332}$ & & & .005 & \begin{tabular}{|l|}
0.10 \\
\end{tabular} & 38 & 8.1 & 730 & 1.25 & 0.13 & 1.22 & 031 & 68.5 & 57 & & 2.37 & 20.2 & 2.81 & 12.60 & 0.19 & 2.2 & 0.23 & \begin{tabular}{|l|l|}
0.044 \\
\end{tabular} & 1.10 & 28.7 & \begin{tabular}{|l|}
23.4 \\
\end{tabular} & & 942 \\
\hline 2033 & & 66220 & .009 & 0.10 & 5.36 & 9.6 & 740 & 1.13 & 0.14 & 1.17 & 0.42 & 65.4 & 17.3 & 66 & 2.47 & 23.0 & 3.15 & 12.45 & 0.22 & 2.2 & 0.10 & \begin{tabular}{|l|l|}
0.043 \\
\end{tabular} & 1.08 & 28.0 & 23.2 & 0.85 & 1210 \\
\hline 2034 & & & .005 & \begin{tabular}{|l}
0.16 \\
\end{tabular} & 13 & 49.0 & 790 & 1.58 & 0.58 & 1.59 & \begin{tabular}{|l|l|}
0.21 \\
\end{tabular} & 100.5 & 13.5 & & 5.31 & 27.3 & 3.32 & 14.15 & 0.24 & 1.8 & 0.14 & 0.056 & 1.34 & 43.9 & 29.6 & 1.04 & 582 \\
\hline 035 & & & 0.008 & 0.11 & 5.88 & 22.2 & 330 & 1.31 & 0.26 & 1.87 & \begin{tabular}{|l|}
0.28 \\
\end{tabular} & 85.8 & 13.3 & & 2.71 & 24.8 & 3.12 & 12.90 & 0.22 & 2.0 & 0.23 & 0.048 & 1.26 & 37.3 & 24.9 & 1.10 & 531 \\
\hline 2036 & 4532 & 38 & 0.005 & 0.15 & 5.87 & 84.9 & 820 & 1.51 & 0.32 & 1.50 & \begin{tabular}{|l|}
0.23 \\
\end{tabular} & 81.9 & 13.1 & & 3.24 & 20.6 & 3.56 & 13.30 & 0.28 & 2.0 & 0.26 & \begin{tabular}{|l|}
0.050 \\
\end{tabular} & 1.26 & 35.6 & \begin{tabular}{|l|}
28.7 \\
\end{tabular} & 1.01 & 492 \\
\hline & & & .005 & 0.11 & 5.82 & & 820 & .36 & 16 & 1.76 & \begin{tabular}{|l|}
0.29 \\
\end{tabular} & 76.5 & & & 2.44 & 22.7 & 3.04 & 13.05 & 0.22 & 1.9 & 0.11 & \begin{tabular}{|l|}
0.046 \\
\end{tabular} & 1.26 & 34.2 & \begin{tabular}{|l|l|}
24.4 \\
\end{tabular} & 1.08 & 538 \\
\hline 2038 & & & .012 & 0.12 & \begin{tabular}{|l|l|}
5.82 \\
\end{tabular} & 41.0 & 660 & 1.93 & 0.93 & 2.04 & 0.20 & 149.5 & 1 & & 6.80 & 31.4 & 3.68 & 13.90 & 0.30 & 2.4 & 0.16 & \begin{tabular}{|l|l|}
0.065 \\
\end{tabular} & 1.31 & 64.5 & 34.5 & 103 & 653 \\
\hline 2040 & & 31 & .005 & 0.12 & 5.72 & 17.3 & 320 & 1.36 & 0.17 & 1.70 & \begin{tabular}{|l|}
0.28 \\
\end{tabular} & 73.2 & 13.3 & 77 & 2.60 & 24.8 & 3.06 & 13.20 & 0.24 & 2.0 & 0.10 & \begin{tabular}{|l|}
0.045 \\
\end{tabular} & 1.24 & 31.9 & \begin{tabular}{|l|}
26.8 \\
\end{tabular} & 1.04 & 451 \\
\hline 44 & & & .005 & 0.13 & 32 & 13.9 & 850 & 1.19 & 0.17 & 1.92 & \begin{tabular}{|l|}
0.24 \\
\end{tabular} & 72.2 & & & 2.4 & 18.9 & 3.07 & 14.95 & 0.18 & 2.0 & 0.25 & \begin{tabular}{|l|}
0.045 \\
\end{tabular} & 1.32 & 36.3 & \begin{tabular}{|l|l|}
25.1 \\
\end{tabular} & & 536 \\
\hline$\overline{42}$ & & & 0.030 & 0.11 & 6.35 & 15.7 & 880 & 1.19 & 0.17 & 1.86 & 0.24 & 66.4 & 12.8 & 85 & 2.52 & 20.0 & 3.11 & 15.15 & 0.19 & 2. & 0.09 & 0.046 & 1.35 & 32.9 & 26.7 & 1.0 & 516 \\
\hline & & & .014 & 0.13 & 27 & 17.8 & 370 & 1.21 & 0.17 & 1.80 & $\begin{array}{l}0.27 \\
\end{array}$ & 68.4 & 13.2 & & 2.68 & 21.4 & 3.10 & 14.90 & 0.18 & $1 . \varepsilon$ & 0.24 & 04.046 & \begin{tabular}{|l}
1.32 \\
\end{tabular} & 33.2 & 26.6 & 1.05 & 576 \\
\hline & & & & 0.13 & & 5 & 30 & 37 & & 84 & 28 & 71.8 & & & 2.5 & 20.2 & 3.20 & 5.25 & 0.19 & 1. & & .045 & 1.35 & 35.6 & 26.1 & & 668 \\
\hline 2046 & & & 0.010 & 0.13 & 6.07 & 16.4 & 840 & 1.28 & 0.19 & 1.62 & \begin{tabular}{|l|}
0.27 \\
\end{tabular} & 57.5 & 12.8 & 71 & 2.67 & 22.4 & 2.92 & 14.80 & 0.20 & 1.7 & 0.27 & 0.043 & 1.33 & 28.7 & \begin{tabular}{|l|}
26.6 \\
\end{tabular} & 1.00 & 503 \\
\hline 477 & & & .013 & 0.08 & .03 & 12.1 & 750 & 1.21 & 0.19 & 1.90 & 0.20 & 90.0 & 11.1 & 10 & 2.42 & 15.2 & 3.04 & 14.30 & 0.23 & 2.6 & 0.07 & .047 & 1.20 & 49.7 & \begin{tabular}{|l|}
23.5 \\
\end{tabular} & 1.03 & 585 \\
\hline & & & .005 & 0.12 & & 3.4 & 60 & .08 & 0.18 & 71 & 0. & 77.4 & 12. & 88 & 2.43 & 17. & 2.92 & 25 & & 2. & 34 & & & 37.5 & 24.0 & & 562 \\
\hline 20 & & 30 & 0.005 & \begin{tabular}{|l|}
0.11 \\
\end{tabular} & 5.94 & 14.1 & 780 & 1.28 & 0.19 & 1.68 & 0.24 & 66.5 & 12.2 & & 2.56 & 18.9 & 2.94 & 14.00 & 0.20 & 1.7 & 0.23 & 0.044 & \begin{tabular}{|l}
1.22 \\
\end{tabular} & 32.8 & 24.3 & 1.00 & 543 \\
\hline 64 & & & 0.007 & 0.26 & 6.30 & 7.2 & 740 & .25 & 0.14 & 1.19 & 0.37 & 56.6 & 12. & & 5.5 & 25.3 & 2.89 & 15.75 & 0.19 & 2.1 & 0.23 & .045 & 1.41 & 27.4 & 32.5 & 0.9 & 538 \\
\hline & & & 0.007 & 0.18 & 91 & 10.5 & 920 & 1.29 & 0.14 & 1.16 & 0.40 & 80.8 & & & 3.95 & 19.9 & 2.97 & 15.85 & 0.19 & 2.7 & 0.1 & \begin{tabular}{|c|}
0.049 \\
\end{tabular} & 1.32 & 37.7 & \begin{tabular}{|l|}
30.1 \\
\end{tabular} & 1. & 637 \\
\hline 67 & & & 0.008 & 0.14 & \begin{tabular}{|l|l|}
6.12 \\
\end{tabular} & 9.0 & 910 & 1.25 & 0.13 & 1.19 & 0.23 & 77.1 & 13.6 & & 3.57 & 18. & 3.11 & 15.75 & 0.20 & 2.4 & 0.19 & 0.049 & 1.36 & 37.7 & \begin{tabular}{|l|}
31.3 \\
\end{tabular} & 1.0 & 544 \\
\hline 688 & & & 0.013 & 0.17 & 10 & 9.7 & 920 & 1.45 & 1.16 & 1.23 & 0.31 & 67. & 111.8 & & $3.7 \varepsilon$ & 22.7 & 2.93 & 15.45 & 0.20 & 2. & 17 & .048 & 1.35 & 32.2 & \begin{tabular}{|l|}
30.2 \\
\end{tabular} & 0.9 & 514 \\
\hline & & & 0.006 & 0.12 & 16 & 11.0 & 880 & 1.33 & 0.15 & 1.44 & 0.31 & 71.4 & 14.5 & & 2.98 & 25.0 & 3.35 & 15.20 & 0.20 & 2.2 & 19 & 0.046 & 1.34 & 33.9 & \begin{tabular}{|l|}
29.1 \\
\end{tabular} & 1. & 553 \\
\hline 2070 & & $\overline{79}$ & 0.006 & 0.12 & $\overline{99}$ & 10.7 & 860 & 1.29 & 0.14 & 1.22 & 0.32 & 60.4 & 15.2 & 14 & 3.00 & 25.3 & 3.30 & 15.25 & 0.20 & 2. & 0.15 & 04.046 & 1.34 & 29.8 & 32.2 & 1.0 & 534 \\
\hline & & & 0.008 & 0.18 & 6.16 & 12.3 & 790 & 1.13 & 0.27 & 1.41 & 0.29 & 63. & 17.4 & & & 26. & 3.0 & 15.45 & 0.21 & 1. & 0.2 & 0.056 & 1.28 & 31.4 & 27.2 & 1.0 & 975 \\
\hline & & & 0.005 & \begin{tabular}{|l|}
0.21 \\
\end{tabular} & & 67 & 90 & 17 & 0.52 & & 025 & 76 & 17 & & $\sqrt{3}$ & 27. & 3.12 & 15.50 & 0.19 & 1. & & .053 & 1.33 & 37.5 & 28.5 & 1.0 & 918 \\
\hline 2078 & & & .005 & 0.16 & 6.07 & 22.6 & 730 & 1.21 & 0.67 & 1.30 & 0.28 & 69.8 & 15.4 & & 3.8 & 26.8 & 3.06 & 15.35 & 0.22 & 2.0 & .20 & .054 & 1.26 & 34.1 & \begin{tabular}{|l|}
27.2 \\
\end{tabular} & \begin{tabular}{|l}
1.02 \\
\end{tabular} & 884 \\
\hline & & & 0.007 & 0.32 & 57 & 14.7 & 880 & 1.24 & 0.24 & 1.26 & 0.29 & 57.4 & 14.2 & & & 32.0 & 2.97 & 16.45 & 0.20 & 1.8 & 23 & .059 & 1.44 & 28.1 & 34.3 & \begin{tabular}{|l}
0.97 \\
\end{tabular} & 1730 \\
\hline 80 & & 55076 & 0.006 & 0.29 & 46 & 33.2 & 820 & 1.38 & 0.88 & 1.27 & 0.35 & 70.7 & 17.2 & $\overline{102}$ & 5.76 & 36.0 & 3.41 & 17.05 & 0.21 & 1.9 & 0.35 & .061 & 1.40 & 33.3 & \begin{tabular}{|l|}
34.0 \\
\end{tabular} & 1.08 & 1415 \\
\hline 2081 & & 255091 & .005 & \begin{tabular}{|l|}
0.19 \\
\end{tabular} & 6.25 & 19.9 & 800 & 1.20 & 0.65 & 1.38 & \begin{tabular}{|l|l|}
0.24 \\
\end{tabular} & 69. & 13. & 97 & 4.3 & 28. & 3.07 & 15.75 & 0.20 & 1. & 0.37 & 0.053 & \begin{tabular}{|l|}
1.34 \\
\end{tabular} & 33.9 & 28.6 & 1.0 & 549 \\
\hline & & & 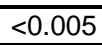 & \begin{tabular}{|l|}
0.73 \\
\end{tabular} & & & & & & & 1.51 & & & & & 24. & 3.12 & 5.55 & 0.20 & & & .085 & 1.29 & 31.7 & 37.5 & 1.1 & 681 \\
\hline & & & 0.010 & \begin{tabular}{|l|}
2.89 \\
\end{tabular} & 96 & 45.3 & 920 & $\overline{25}$ & 0.24 & 1.34 & 6.58 & 71.6 & 15.4 & 100 & 5.81 & 35. & 3.61 & 16.15 & 0.24 & 2.1 & 1.02 & 2.210 & 1.42 & 34.1 & \begin{tabular}{|l|}
32.0 \\
\end{tabular} & 1.06 & 1630 \\
\hline 2122 & & 7289287 & 0.005 & 0.12 & \begin{tabular}{|l|}
6.16 \\
\end{tabular} & 10.3 & 960 & 1.01 & 0.12 & 1.85 & \begin{tabular}{|l|}
0.33 \\
\end{tabular} & 56.6 & 20.4 & 75 & 2.13 & 34.5 & 4.67 & 15.85 & 0.22 & 2.6 & 0.10 & .057 & 0.88 & 27.7 & \begin{tabular}{|l|}
20.6 \\
\end{tabular} & \begin{tabular}{|l|l|} 
& \\
\end{tabular} & 821 \\
\hline & & & & & & & & & & & & & & & & & & & & & & .058 & 1.26 & 32.1 & \begin{tabular}{|l|}
31.9 \\
\end{tabular} & \begin{tabular}{|l|}
0.88 \\
\end{tabular} & 644 \\
\hline 2805 & 36043 & 7255849 & 0.057 & 0.56 & 6.17 & 10.0 & 680 & 1.12 & 2.17 & 0.75 & 0.65 & 63.8 & 13.6 & 89 & 6.65 & 39.0 & 3.52 & 15.85 & 0.22 & 2.2 & 0.22 & 0.084 & 1.32 & 31.1 & \begin{tabular}{|l|}
38.9 \\
\end{tabular} & \begin{tabular}{|l|}
0.82 \\
\end{tabular} & 610 \\
\hline 2806 & & & 0.016 & 0.23 & 6.35 & 11.9 & 850 & 1.39 & 0.19 & 1.3 & \begin{tabular}{|l|}
0.63 \\
\end{tabular} & 64 & 14.5 & 8 & 3. & 22.1 & 3.15 & 15.85 & 0.24 & 2.0 & 0.13 & .049 & 1.30 & 32.9 & \begin{tabular}{|l|}
29.8 \\
\end{tabular} & 0.99 & 755 \\
\hline & & & 0.010 & 0.54 & 5.37 & 20.3 & & 1. & & 2.02 & & 56. & & 8 & & 67.1 & 3.02 & & 0.24 & 1.8 & & .058 & 1.06 & 30.3 & \begin{tabular}{|l|}
36.1 \\
\end{tabular} & \begin{tabular}{|l|}
1.02 \\
\end{tabular} & 749 \\
\hline 4201 & 15992 & 7287814 & 0.013 & 0.14 & \begin{tabular}{|l|}
5.79 \\
\end{tabular} & 9.6 & 910 & 1.16 & 0.16 & 1.82 & \begin{tabular}{|l|}
0.62 \\
\end{tabular} & 53.5 & 13.2 & 75 & 4.03 & 28.7 & 3.13 & 14.50 & 0.23 & 2.0 & 0.12 & 0.046 & 1.12 & 27.0 & 26.7 & 0.90 & 605 \\
\hline
\end{tabular}


Table 1. Location and New Trace Element Geochemical Results for 902 Stream-Sediment Samples collected in the Livengood Area, Livengood Quadrangle, Alaska IS = Insufficient Sample for Analysis

\begin{tabular}{|c|c|c|c|c|c|c|c|c|c|c|c|c|c|c|c|c|c|c|c|c|c|c|c|c|c|c|}
\hline AMPLE & $\begin{array}{l}\text { Mo } \\
\text { ppm }\end{array}$ & $\begin{array}{c}\mathrm{Na} \\
\%\end{array}$ & $\begin{array}{l}\mathrm{Nb} \\
\mathrm{ppm}\end{array}$ & $\begin{array}{r}\mathrm{Ni} \\
\mathrm{ppm}\end{array}$ & $\begin{array}{c}\mathrm{P} \\
\mathrm{ppm}\end{array}$ & $\begin{array}{l}\mathrm{Pb} \\
\mathrm{ppm}\end{array}$ & $\begin{array}{l}\mathrm{Rb} \\
\mathrm{ppm}\end{array}$ & $\begin{array}{l}\mathrm{Re} \\
\mathrm{ppm}\end{array}$ & $\begin{array}{l}\mathrm{S} \\
\%\end{array}$ & $\begin{array}{l}\mathrm{Sb} \\
\mathrm{ppm}\end{array}$ & $\begin{array}{c}\text { Se } \\
\mathrm{ppm}\end{array}$ & $\begin{array}{l}S^{S n^{*}} \\
\text { ppm }\end{array}$ & $\begin{array}{c}\mathrm{Sr} \\
\mathrm{ppm}\end{array}$ & $\begin{array}{l}\mathrm{Ta}^{*} \\
\mathrm{ppm}\end{array}$ & $\begin{array}{c}\text { Te } \\
\text { ppm }\end{array}$ & $\begin{array}{c}\text { Th } \\
\text { ppm }\end{array}$ & $\begin{array}{l}\mathrm{Ti}^{\star} \\
\%\end{array}$ & \begin{tabular}{c|}
$\mathrm{Tl}$ \\
$\mathrm{ppm}$
\end{tabular} & $\begin{array}{c}\mathrm{U} \\
\mathrm{ppm}\end{array}$ & $\begin{array}{c}\mathrm{V} \\
\mathrm{ppm}\end{array}$ & $\begin{array}{l}W^{*} \\
\text { ppm }\end{array}$ & \begin{tabular}{|c|}
$\mathrm{Y}$ \\
$\mathrm{ppm}$ \\
\end{tabular} & $\begin{array}{l}\mathrm{Zn} \\
\mathrm{ppm}\end{array}$ & $\begin{array}{l}Z^{*} r^{*} \\
\mathrm{ppm}\end{array}$ & $\begin{array}{c}\text { Pulp Wt } \\
\text { grams }\end{array}$ & $\begin{array}{c}\text { Lab } \\
\text { Report }\end{array}$ \\
\hline 2025 & 1.27 & 1.15 & 9.2 & 23.7 & 610 & 12.4 & 61.7 & $<0.002$ & 0.03 & 0.84 & $<1$ & 1.50 & 191.0 & 0.65 & 0.05 & 6.9 & 0.391 & 0.39 & 2.2 & 113 & 1.3 & 14.4 & 74 & 71.0 & 27 & FA04030732 \\
\hline 2026 & 1.32 & 1.26 & 10.2 & 26.9 & 750 & 13.0 & 56.9 & $<0.002$ & 0.03 & 1.05 & $<1$ & 1.60 & 216.0 & 0.70 & 0.05 & 8.4 & 0.437 & 0.36 & 2.5 & 118 & 1.1 & \begin{tabular}{|l|}
17.0 \\
\end{tabular} & 66 & 72.9 & 35 & FA04030732 \\
\hline 2027 & 1.31 & 1.24 & \begin{tabular}{|l|}
9.8 \\
\end{tabular} & 28.6 & 740 & 13.1 & 59.4 & $<0.002$ & 0.03 & 0.99 & $<1$ & 1.60 & 217.0 & 0.76 & $<0.05$ & 8.3 & 0.427 & 0.36 & 2.3 & 118 & 1.2 & 17.2 & 78 & 70.7 & 22 & FA04030732 \\
\hline 2028 & 1.09 & 1.19 & 8.6 & 23.7 & 690 & 11.3 & 60.8 & $<0.002$ & 0.04 & 0.87 & $<1$ & 1.50 & 212.0 & 0.62 & $<0.05$ & 6.5 & 0.367 & 0.39 & 2.1 & 108 & 0.9 & 15.7 & 70 & 63.0 & 23 & FA04030732 \\
\hline 2029 & 1.27 & 1.15 & 9.5 & 26.0 & 700 & 12.7 & 58.1 & $<0.002$ & 0.03 & 1.04 & $<1$ & 1.70 & 199.5 & 0.70 & 0.05 & 7.3 & 0.409 & 0.36 & 2.3 & 112 & 1.3 & 16.0 & 74 & 71.0 & 20 & FA04030732 \\
\hline 2030 & 1.07 & 1.19 & 10.1 & 25.8 & 670 & 12.2 & 57.0 & $<0.002$ & 0.03 & 0.90 & $<1$ & 1.50 & 204.0 & 0.72 & $<0.05$ & 8.2 & 0.429 & 0.37 & 2.4 & 110 & 1.2 & 16.6 & 67 & 75.6 & 25 & FA04030732 \\
\hline 2032 & 1.06 & 1.24 & 9.3 & 26.3 & 690 & 11.6 & 56.9 & $<0.002$ & 0.03 & 0.91 & $<1$ & 1.50 & 214.0 & 0.71 & 0.05 & 7.4 & 0.400 & 0.33 & 2.2 & 106 & 1.0 & \begin{tabular}{|l|}
16.8 \\
\end{tabular} & 71 & 72.5 & 24 & FA04030732 \\
\hline 2033 & 1.18 & 1.13 & 8.6 & 26.9 & 740 & 12.5 & 56.4 & $<0.002$ & 0.03 & 0.96 & $<1$ & 1.40 & 199.0 & 0.64 & 0.05 & 7.1 & 0.394 & 0.34 & 2.1 & 110 & 0.9 & 17.2 & 78 & 69.4 & 43 & FA04030732 \\
\hline 2034 & 0.85 & 1.37 & 16.0 & 29.8 & 1190 & 15.5 & 85.0 & $<0.002$ & 0.02 & 1.39 & $<1$ & 2.50 & 290.0 & 1.20 & $<0.05$ & 13.0 & 0.442 & 0.51 & 3.1 & 114 & 1.4 & 18.3 & 74 & 54.6 & 32 & FA04030732 \\
\hline 2035 & 0.67 & 1.38 & 11.0 & 33.9 & 900 & 13.1 & 64.4 & $<0.002$ & 0.02 & 1.16 & $<1$ & 1.70 & 258.0 & 0.79 & $<0.05$ & 10.9 & 0.440 & 0.37 & 2.8 & 113 & 1.2 & 18.2 & 74 & 64.0 & 36 & FA04030732 \\
\hline 2036 & 1.11 & 1.30 & 11.2 & 31.9 & 960 & 14.4 & 70.8 & $<0.002$ & 0.03 & 1.18 & $<1$ & 1.70 & 241.0 & 0.79 & $<0.05$ & 10.4 & 0.418 & 0.39 & 2.8 & 114 & 1.4 & 17.4 & 77 & 62.2 & 50 & FA04030732 \\
\hline 2037 & 0.78 & 1.40 & 10.1 & 36.7 & 850 & 12.4 & 61.3 & $<0.002$ & 0.02 & 1.18 & $<1$ & 1.50 & 251.0 & 0.75 & $<0.05$ & 8.5 & 0.414 & 0.34 & 2.3 & 113 & 1.0 & 18.0 & 79 & 59.3 & 71 & FA04030732 \\
\hline 2038 & 1.12 & 1.49 & 37.6 & 27.6 & 2040 & 15.4 & 96.4 & $\mid<0.002$ & 0.03 & 1.32 & $<1$ & 6.70 & 356.0 & 2.98 & 0.05 & 20.6 & 0.614 & 0.54 & 11.6 & 122 & 2.7 & 25.8 & 72 & 70.7 & 2 & FA04030733 \\
\hline 2040 & 0.75 & 1.35 & 10.0 & 34.8 & 850 & 13.0 & 62.8 & $<0.002$ & 0.03 & 1.15 & $<1$ & 1.60 & 252.0 & 0.77 & $<0.05$ & 8.9 & 0.402 & 0.37 & 2.6 & 110 & 1.0 & 17.3 & 74 & 63.4 & 60 & FA04030733 \\
\hline 2041 & 0.69 & 1.56 & 11.2 & 32.4 & 900 & 11.6 & 59.4 & $<0.002$ & 0.02 & 1.17 & $<1$ & 1.80 & 262.0 & 0.82 & $<0.05$ & 9.4 & 0.443 & 0.40 & 2.4 & 114 & 1.5 & 18.3 & 82 & 65.1 & & FA04030733 \\
\hline 2042 & 0.70 & 1.52 & 10.5 & 32.4 & 880 & 12.0 & 61.1 & $<0.002$ & 0.02 & 1.12 & $<1$ & 1.60 & 258.0 & 0.79 & $<0.05$ & 8.4 & 0.424 & 0.39 & 2.4 & 114 & 1.3 & 17.4 & 88 & 64.4 & 71 & FA04030733 \\
\hline 2043 & 0.76 & 1.49 & \begin{tabular}{|l|}
10.6 \\
\end{tabular} & 33.3 & 890 & 12.6 & 62.9 & $<0.002$ & 0.03 & 1.14 & $<1$ & 1.90 & 255.0 & \begin{tabular}{|l|}
0.81 \\
\end{tabular} & $<0.05$ & 9.0 & 0.412 & 0.40 & 2.4 & 114 & 1.2 & 17.7 & 83 & 62.0 & 82 & FA04030733 \\
\hline 2045 & 0.86 & 1.51 & 11.2 & 32.3 & 930 & 12.0 & 62.2 & $<0.002$ & 0.03 & 1.16 & $<1$ & 1.80 & 260.0 & 0.84 & $<0.05$ & 9.1 & 0.444 & 0.38 & 2.4 & 118 & 1.2 & 17.9 & & 65 & & 30733 \\
\hline 2046 & 0.70 & 1.42 & \begin{tabular}{|l|}
9.1 \\
\end{tabular} & 32.9 & 780 & 11.9 & 62.8 & $<0.002$ & 0.03 & 1.10 & $<1$ & 1.70 & 239.0 & 0.64 & $<0.05$ & 7.6 & 0.360 & 0.40 & 2.2 & 108 & 1.1 & 16.2 & 86 & 56.2 & 70 & FA04030733 \\
\hline 2047 & 0.67 & 1.46 & 20.3 & 28.4 & 1000 & 11.4 & 58.0 & $<0.002$ & 0.02 & 1.05 & $<1$ & 2.50 & 251.0 & 1.66 & $<0.05$ & 13.6 & 0.596 & 0.37 & 3.2 & 119 & 2.0 & 20.5 & 70 & 86.1 & 48 & FA04030733 \\
\hline 2048 & 0.62 & 1.42 & 12.2 & 29.8 & 860 & 11.0 & 57.7 & $<0.002$ & 0.02 & 1.03 & $<1$ & 1.80 & 236.0 & 0.90 & $<0.05$ & 10.0 & 0.448 & 0.38 & 2.6 & 111 & 1.4 & 18.7 & 76 & 70.6 & 98 & FA04030733 \\
\hline 2050 & 0.59 & 1.38 & 10.8 & 30.4 & 880 & 11.1 & 58.1 & $<0.002$ & 0.38 & 1.19 & $<1$ & 1.90 & 232.0 & 0.87 & $<0.05$ & 9.8 & 0.398 & 0.39 & 2.2 & 108 & 1.3 & 16.5 & 73 & 60.6 & 77 & FA04030733 \\
\hline 2064 & 0.68 & 0.96 & 8.9 & 53.5 & 700 & 10.5 & 72.9 & $<0.002$ & 0.04 & 1.02 & $<1$ & 1.70 & 150.0 & 0.61 & $<0.05$ & 7.3 & 0.395 & 0.59 & 2.0 & 136 & 1.4 & 19.0 & 98 & 78.1 & $2 \mathrm{2c}$ & FA04030733 \\
\hline 2065 & 1.26 & 1.10 & 12.4 & 68.3 & 630 & 11.6 & 70.4 & $<0.002$ & 0.03 & 1.37 & $<1$ & 1.80 & 159.0 & 0.97 & \begin{tabular}{|l|}
0.05 \\
\end{tabular} & 9.0 & 0.423 & 0.56 & 2.4 & 130 & 1.1 & 19.6 & 91 & 97.0 & 24 & FA04030733 \\
\hline 2067 & 1.18 & 1.16 & 11.6 & 59.2 & 680 & 11.5 & 68.4 & $<0.002$ & 0.02 & 1.23 & $<1$ & 1.80 & 171.5 & 0.82 & $<0.05$ & 9.2 & 0.450 & 0.51 & 2.3 & 136 & 1.0 & 18.5 & 98 & 89.3 & 41 & FA04030733 \\
\hline 2068 & 1.10 & 1.16 & 10.2 & 51.3 & 700 & 11.9 & 68.7 & $<0.002$ & 0.03 & 1.22 & $<1$ & 1.80 & 182.5 & 0.72 & 0.05 & 8.1 & 0.404 & 0.57 & 2.5 & 128 & 1.1 & 18.1 & 90 & 84.9 & 2 & FA04030733 \\
\hline 2069 & 1.08 & 1.28 & 10.4 & 56.3 & 820 & 12.0 & 63.4 & $<0.002$ & 0.02 & 1.24 & $<1$ & 1.60 & 203.0 & 0.72 & $<0.05$ & 8.3 & 0.420 & 0.44 & 2.2 & 128 & 1.1 & 18.6 & 99 & 78.1 & 7 & FA04030733 \\
\hline 2070 & 1.11 & 1.19 & \begin{tabular}{|l|}
10.1 \\
\end{tabular} & 61.1 & 730 & 12.3 & 65.3 & $<0.002$ & 0.02 & 1.25 & $<1$ & 1.60 & 182.0 & 0.75 & $<0.05$ & 7.8 & 0.417 & 0.44 & 2.1 & 132 & 1.1 & 17.6 & 101 & 82.5 & 30 & FA04030733 \\
\hline 2075 & 0.86 & 1.24 & 9.2 & 36.9 & 750 & 11.6 & 65.2 & $<0.002$ & 0.03 & 1.37 & $<1$ & 2.10 & 198.0 & 0.67 & 0.05 & 7.7 & 0.406 & 0.41 & 2.0 & 122 & 1. & 16.3 & 84 & 65.5 & 5 & A04030733 \\
\hline 2077 & 0.87 & 1.22 & 9.2 & 36.8 & 770 & 11.6 & 67.4 & $<0.002$ & 0.03 & 1.32 & $<1$ & 2.20 & 193.0 & 0.69 & 0.05 & 7.9 & 0.412 & 0.45 & 2.1 & 126 & 1.5 & \begin{tabular}{|l|}
16.2 \\
\end{tabular} & 78 & 63.2 & 4 & FA04030733 \\
\hline 2078 & 0.86 & 1.23 & 10.0 & 36.4 & 740 & 12.4 & 66.6 & $<0.002$ & 0.03 & 1.52 & $<1$ & 2.40 & 187.0 & 0.70 & 0.05 & 8.6 & 0.437 & 0.47 & 2.2 & 122 & 1.9 & 16.2 & 71 & 68.3 & 39 & FA04030733 \\
\hline 2079 & 0.91 & 1.22 & \begin{tabular}{|l|}
9.0 \\
\end{tabular} & 38.3 & 740 & 11.4 & 75.1 & $<0.002$ & 0.02 & 1.42 & $<1$ & 2.40 & 192.0 & 0.65 & 0.05 & 7.3 & 0.368 & 0.48 & 2.0 & 124 & 1.2 & \begin{tabular}{|l|}
15.7 \\
\end{tabular} & 77 & 64.2 & 35 & FA04030733 \\
\hline 2080 & 1.02 & 1.12 & 10.2 & 44.6 & 810 & 13.1 & 78.7 & $<0.002$ & 0.03 & 1.74 & $<1$ & 2.90 & 177.5 & 0.75 & 0.06 & 8.1 & 0.431 & 0.55 & 2.1 & 137 & 1.9 & 17.0 & 82 & 66.8 & 27 & FA04030733 \\
\hline 2081 & 0.69 & 1.24 & 9.9 & 37.1 & 720 & 12.2 & 70.4 & $<0.002$ & 0.03 & 1.50 & $<1$ & 2.50 & 192.5 & 0.76 & 0.06 & 8.1 & 0.431 & 0.47 & 2.1 & 124 & 1.6 & 16.4 & 75 & 63.3 & 48 & FA04030733 \\
\hline 2083 & 0.49 & 1.36 & 9.6 & 35.5 & 730 & 40.4 & 61.9 & $<0.002$ & 0.02 & 3.83 & $<1$ & 1.90 & 199.0 & 0.70 & $<0.05$ & 8.2 & 0.447 & 0.43 & 1.9 & 128 & 1.0 & \begin{tabular}{|l|}
16.5 \\
\end{tabular} & 176 & 58.7 & & $=\mathrm{A} 04030733$ \\
\hline 2085 & 0.81 & 1.22 & 10.4 & 43.5 & 980 & 215.0 & 75.4 & $<0.002$ & 0.04 & 13.60 & $<1$ & 3.80 & 197.5 & 0.76 & 0.05 & 8.0 & 0.464 & 0.51 & 2.1 & 134 & 1.6 & 19.3 & 320 & 74.1 & 39 & FA04030733 \\
\hline 2122 & 1.00 & 1.63 & 12.2 & 33.2 & 620 & 11.3 & 43.2 & $<0.002$ & 0.02 & 0.89 & $<1$ & 1.70 & 201.0 & 0.85 & $<0.05$ & 6.2 & 0.852 & 0.25 & 1.7 & 203 & 0.9 & \begin{tabular}{|l|}
19.8 \\
\end{tabular} & 95 & 90.3 & 55 & FA04030733 \\
\hline 2804 & 1.09 & 1.03 & 10.2 & 31.8 & 670 & 14.2 & 74.9 & & 0.03 & 1.19 & $<1$ & 3.90 & 160.0 & 0.77 & \begin{tabular}{|l|}
0.07 \\
\end{tabular} & 8.1 & 0.432 & 0.57 & 2.2 & & & \begin{tabular}{|l|l|}
14.4 \\
\end{tabular} & 91 & 72.2 & & FA04030733 \\
\hline 2805 & 1.09 & 0.83 & 10.0 & 33.3 & 790 & 15.1 & 76.4 & \begin{tabular}{|c|}
$<0.002$ \\
\end{tabular} & 0.03 & 1.28 & $<1$ & 3.70 & 162.0 & 0.73 & 0.06 & 8.0 & 0.454 & 0.74 & 2.1 & 137 & 2.7 & 13.9 & 97 & 74.7 & 28 & FA04030733 \\
\hline 2806 & 0.82 & 1.26 & 10.2 & 32.7 & 690 & 20.0 & 67.7 & $<<0.002$ & 0.02 & 1.25 & $<1$ & 2.00 & 196.0 & 0.77 & 0.05 & 8.8 & 0.426 & 0.45 & 2.2 & 122 & 1.4 & 16.2 & 86 & 71.6 & 42 & FA04030733 \\
\hline 3171 & 0.97 & 0.86 & 7.4 & 49.2 & 860 & 19.1 & 56.5 & $<0.002$ & 0.07 & 2.92 & 1 & 1.50 & 213.0 & 0.56 & 0.05 & 6.1 & 0.346 & 0.44 & 2.2 & 122 & 0.8 & \begin{tabular}{|l|}
24.8 \\
\end{tabular} & 105 & 61.5 & 1 & FA04030733 \\
\hline 4201 & 0.95 & 1.12 & 9.0 & 32.9 & 740 & 12.3 & 63.3 & \begin{tabular}{|c|}
$<0.002$ \\
\end{tabular} & 0.05 & 16.00 & $<1$ & 1.60 & 189.0 & 0.61 & 0.05 & 7.4 & 0.435 & 0.43 & 2.1 & 134 & 1.0 & 17.8 & 108 & 66.5 & 19 & FA04030733 \\
\hline
\end{tabular}


Table 2. Limits, digestive methods, and analytical methods for trace-element geochemical analysis.

Analytical methods: AAS = Atomic Absorption Spectroscopy, ICP-MS = Inductively Coupled Plasma-Mass Spectroscopy, ICP-AES = Inductively Coupled Plasma-Atomic Emission Spectroscopy. $\quad$ *-Possible Incomplete Digestion.

\begin{tabular}{|c|c|c|c|c|}
\hline Element & Digestion Method & Analytical Method & Lower Detection Limit & Upper Detection Limit \\
\hline $\mathrm{Au}$ & Fire Assay & AAS & $0.005 \mathrm{ppm}$ & 10 ppm \\
\hline $\mathrm{Ag}$ & $\mathrm{HF}-\mathrm{HNO}_{3}-\mathrm{HClO}_{4}$ acid digestion \& $\mathrm{HCl}$ leach & ICP-AES \& ICP-MS & $0.01 \mathrm{ppm}$ & $100 \mathrm{ppm}$ \\
\hline $\mathrm{Al}$ & $\mathrm{HF}-\mathrm{HNO}_{3}-\mathrm{HClO}_{4}$ acid digestion \& $\mathrm{HCl}$ leach & ICP-AES & $0.01 \%$ & $25 \%$ \\
\hline As & $\mathrm{HF}-\mathrm{HNO}_{3}-\mathrm{HClO}_{4}$ acid digestion \& $\mathrm{HCl}$ leach & ICP-AES \& ICP-MS & $0.2 \mathrm{ppm}$ & $10,000 \mathrm{ppm}$ \\
\hline $\mathrm{Ba}^{*}$ & $\mathrm{HF}-\mathrm{HNO}_{3}-\mathrm{HClO}_{4}$ acid digestion \& $\mathrm{HCl}$ leach & ICP-AES & $10 \mathrm{ppm}$ & $10,000 \mathrm{ppm}$ \\
\hline $\mathrm{Be}$ & $\mathrm{HF}-\mathrm{HNO}_{3}-\mathrm{HClO}_{4}$ acid digestion \& $\mathrm{HCl}$ leach & ICP-AES \& ICP-MS & $0.05 \mathrm{ppm}$ & $1000 \mathrm{ppm}$ \\
\hline $\mathrm{Bi}$ & $\mathrm{HF}-\mathrm{HNO}_{3}-\mathrm{HClO}_{4}$ acid digestion \& $\mathrm{HCl}$ leach & ICP-AES \& ICP-MS & $0.01 \mathrm{ppm}$ & $10,000 \mathrm{ppm}$ \\
\hline $\mathrm{Ca}$ & $\mathrm{HF}-\mathrm{HNO}_{3}-\mathrm{HClO}_{4}$ acid digestion \& $\mathrm{HCl}$ leach & ICP-AES & $0.01 \%$ & $25 \%$ \\
\hline $\mathrm{Cd}$ & $\mathrm{HF}-\mathrm{HNO}_{3}-\mathrm{HClO}_{4}$ acid digestion \& $\mathrm{HCl}$ leach & ICP-AES \& ICP-MS & $0.02 \mathrm{ppm}$ & 500 ppm \\
\hline $\mathrm{Ce}$ & $\mathrm{HF}-\mathrm{HNO}_{3}-\mathrm{HClO}_{4}$ acid digestion \& $\mathrm{HCl}$ leach & ICP-MS & $0.01 \mathrm{ppm}$ & 500 ppm \\
\hline Co & $\mathrm{HF}-\mathrm{HNO}_{3}-\mathrm{HClO}_{4}$ acid digestion \& $\mathrm{HCl}$ leach & ICP-AES \& ICP-MS & $0.1 \mathrm{ppm}$ & $10,000 \mathrm{ppm}$ \\
\hline $\mathrm{Cr}^{*}$ & $\mathrm{HF}-\mathrm{HNO}_{3}-\mathrm{HClO}_{4}$ acid digestion \& $\mathrm{HCl}$ leach & ICP-AES & $1 \mathrm{ppm}$ & $10,000 \mathrm{ppm}$ \\
\hline Cs & $\mathrm{HF}-\mathrm{HNO}_{3}-\mathrm{HClO}_{4}$ acid digestion \& $\mathrm{HCl}$ leach & ICP-MS & $0.05 \mathrm{ppm}$ & 500 ppm \\
\hline $\mathrm{Cu}$ & $\mathrm{HF}-\mathrm{HNO}_{3}-\mathrm{HClO}_{4}$ acid digestion \& $\mathrm{HCl}$ leach & ICP-AES & $0.2 \mathrm{ppm}$ & $10,000 \mathrm{ppm}$ \\
\hline $\mathrm{Fe}$ & $\mathrm{HF}-\mathrm{HNO}_{3}-\mathrm{HClO}_{4}$ acid digestion \& $\mathrm{HCl}$ leach & ICP-AES & $0.01 \%$ & $25 \%$ \\
\hline $\mathrm{Ga}$ & $\mathrm{HF}-\mathrm{HNO}_{3}-\mathrm{HClO}_{4}$ acid digestion \& $\mathrm{HCl}$ leach & ICP-MS & $0.05 \mathrm{ppm}$ & 500 ppm \\
\hline $\mathrm{Ge}$ & $\mathrm{HF}-\mathrm{HNO}_{3}-\mathrm{HClO}_{4}$ acid digestion \& $\mathrm{HCl}$ leach & ICP-MS & $0.05 \mathrm{ppm}$ & 500 ppm \\
\hline $\mathrm{Hf}$ & $\mathrm{HF}-\mathrm{HNO}_{3}-\mathrm{HClO}_{4}$ acid digestion \& $\mathrm{HCl}$ leach & ICP-MS & $0.1 \mathrm{ppm}$ & 500 ppm \\
\hline $\mathrm{Hg}$ & Cold Vapor Nitric Aqua Regia digestion & AAS & $10 \mathrm{ppm}$ & $100 \mathrm{ppm}$ \\
\hline In & $\mathrm{HF}-\mathrm{HNO}_{3}-\mathrm{HClO}_{4}$ acid digestion \& $\mathrm{HCl}$ leach & ICP-MS & $0.005 \mathrm{ppm}$ & 500 ppm \\
\hline $\mathrm{K}$ & $\mathrm{HF}-\mathrm{HNO}_{3}-\mathrm{HClO}_{4}$ acid digestion \& $\mathrm{HCl}$ leach & ICP-AES & $0.01 \%$ & $10 \%$ \\
\hline La & $\mathrm{HF}-\mathrm{HNO}_{3}-\mathrm{HClO}_{4}$ acid digestion \& $\mathrm{HCl}$ leach & ICP-MS & $0.5 \mathrm{ppm}$ & 500 ppm \\
\hline $\mathrm{Li}$ & $\mathrm{HF}-\mathrm{HNO}_{3}-\mathrm{HClO}_{4}$ acid digestion \& $\mathrm{HCl}$ leach & ICP-MS & $0.2 \mathrm{ppm}$ & 500 ppm \\
\hline $\mathrm{Mg}$ & $\mathrm{HF}-\mathrm{HNO}_{3}-\mathrm{HClO}_{4}$ acid digestion \& $\mathrm{HCl}$ leach & ICP-AES & $0.01 \%$ & $15 \%$ \\
\hline $\mathrm{Mn}$ & $\mathrm{HF}-\mathrm{HNO}_{3}-\mathrm{HClO}_{4}$ acid digestion \& $\mathrm{HCl}$ leach & ICP-AES & $5 \mathrm{ppm}$ & $10,000 \mathrm{ppm}$ \\
\hline Mo & $\mathrm{HF}-\mathrm{HNO}_{3}-\mathrm{HClO}_{4}$ acid digestion \& $\mathrm{HCl}$ leach & ICP-AES & $0.05 \mathrm{ppm}$ & $10,000 \mathrm{ppm}$ \\
\hline $\mathrm{Na}$ & $\mathrm{HF}-\mathrm{HNO}_{3}-\mathrm{HClO}_{4}$ acid digestion \& $\mathrm{HCl}$ leach & ICP-AES & $0.01 \%$ & $10 \%$ \\
\hline $\mathrm{Nb}$ & $\mathrm{HF}-\mathrm{HNO}_{3}-\mathrm{HClO}_{4}$ acid digestion \& $\mathrm{HCl}$ leach & ICP-MS & $0.1 \mathrm{ppm}$ & 500 ppm \\
\hline $\mathrm{Ni}$ & $\mathrm{HF}-\mathrm{HNO}_{3}-\mathrm{HClO}_{4}$ acid digestion \& $\mathrm{HCl}$ leach & ICP-AES \& ICP-MS & $0.2 \mathrm{ppm}$ & $10,000 \mathrm{ppm}$ \\
\hline $\mathrm{P}$ & $\mathrm{HF}-\mathrm{HNO}_{3}-\mathrm{HClO}_{4}$ acid digestion \& $\mathrm{HCl}$ leach & ICP-AES & $10 \mathrm{ppm}$ & $10,000 \mathrm{ppm}$ \\
\hline $\mathrm{Pb}$ & $\mathrm{HF}-\mathrm{HNO}_{3}-\mathrm{HClO}_{4}$ acid digestion \& $\mathrm{HCl}$ leach & ICP-AES \& ICP-MS & $0.5 \mathrm{ppm}$ & $10,000 \mathrm{ppm}$ \\
\hline $\mathrm{Rb}$ & $\mathrm{HF}-\mathrm{HNO}_{3}-\mathrm{HClO}_{4}$ acid digestion \& $\mathrm{HCl}$ leach & ICP-MS & $0.1 \mathrm{ppm}$ & 500 ppm \\
\hline $\mathrm{Re}$ & $\mathrm{HF}-\mathrm{HNO}_{3}-\mathrm{HClO}_{4}$ acid digestion \& $\mathrm{HCl}$ leach & ICP-MS & $0.002 \mathrm{ppm}$ & $50 \mathrm{ppm}$ \\
\hline $\mathrm{S}$ & $\mathrm{HF}-\mathrm{HNO}_{3}-\mathrm{HClO}_{4}$ acid digestion \& $\mathrm{HCl}$ leach & ICP-AES & $0.01 \%$ & $10 \%$ \\
\hline $\mathrm{Sb}$ & $\mathrm{HF}-\mathrm{HNO}_{3}-\mathrm{HClO}_{4}$ acid digestion \& $\mathrm{HCl}$ leach & ICP-MS & $0.05 \mathrm{ppm}$ & $1,000 \mathrm{ppm}$ \\
\hline $\mathrm{Se}$ & $\mathrm{HF}-\mathrm{HNO}_{3}-\mathrm{HClO}_{4}$ acid digestion \& $\mathrm{HCl}$ leach & ICP-MS & $1 \mathrm{ppm}$ & $1,000 \mathrm{ppm}$ \\
\hline $\mathrm{Sn}^{*}$ & $\mathrm{HF}-\mathrm{HNO}_{3}-\mathrm{HClO}_{4}$ acid digestion \& $\mathrm{HCl}$ leach & ICP-MS & $0 . .2 \mathrm{ppm}$ & 500 ppm \\
\hline $\mathrm{Sr}$ & $\mathrm{HF}-\mathrm{HNO}_{3}-\mathrm{HClO}_{4}$ acid digestion \& $\mathrm{HCl}$ leach & ICP-AES \& ICP-MS & $0.2 \mathrm{ppm}$ & $10,000 \mathrm{ppm}$ \\
\hline $\mathrm{Ta}^{*}$ & $\mathrm{HF}-\mathrm{HNO}_{3}-\mathrm{HClO}_{4}$ acid digestion \& $\mathrm{HCl}$ leach & ICP-MS & $0.05 \mathrm{ppm}$ & $100 \mathrm{ppm}$ \\
\hline Te & $\mathrm{HF}-\mathrm{HNO}_{3}-\mathrm{HClO}_{4}$ acid digestion \& $\mathrm{HCl}$ leach & ICP-MS & $0.05 \mathrm{ppm}$ & 500 ppm \\
\hline Th & $\mathrm{HF}-\mathrm{HNO}_{3}-\mathrm{HClO}_{4}$ acid digestion \& $\mathrm{HCl}$ leach & ICP-MS & $0.2 \mathrm{ppm}$ & 500 ppm \\
\hline $\mathrm{Ti}^{*}$ & $\mathrm{HF}-\mathrm{HNO}_{3}-\mathrm{HClO}_{4}$ acid digestion \& $\mathrm{HCl}$ leach & ICP-AES \& ICP-MS & $0.005 \%$ & $10 \%$ \\
\hline $\mathrm{Tl}$ & $\mathrm{HF}-\mathrm{HNO}_{3}-\mathrm{HClO}_{4}$ acid digestion \& $\mathrm{HCl}$ leach & ICP-MS & $0.02 \mathrm{ppm}$ & 500 ppm \\
\hline$U$ & $\mathrm{HF}-\mathrm{HNO}_{3}-\mathrm{HClO}_{4}$ acid digestion \& $\mathrm{HCl}$ leach & ICP-MS & $0.1 \mathrm{ppm}$ & 500 ppm \\
\hline $\mathrm{V}$ & $\mathrm{HF}-\mathrm{HNO}_{3}-\mathrm{HClO}_{4}$ acid digestion \& $\mathrm{HCl}$ leach & ICP-AES & $1 \mathrm{ppm}$ & $10,000 \mathrm{ppm}$ \\
\hline$W^{*}$ & $\mathrm{HF}-\mathrm{HNO}_{3}-\mathrm{HClO}_{4}$ acid digestion \& $\mathrm{HCl}$ leach & ICP-AES \& ICP-MS & $0.1 \mathrm{ppm}$ & $10,000 \mathrm{ppm}$ \\
\hline $\mathrm{Y}$ & $\mathrm{HF}-\mathrm{HNO}_{3}-\mathrm{HClO}_{4}$ acid digestion \& $\mathrm{HCl}$ leach & ICP-MS & $0.1 \mathrm{ppm}$ & $500 \mathrm{ppm}$ \\
\hline $\mathrm{Zn}$ & $\mathrm{HF}-\mathrm{HNO}_{3}-\mathrm{HClO}_{4}$ acid digestion \& $\mathrm{HCl}$ leach & ICP-AES & $2 \mathrm{ppm}$ & $10,000 \mathrm{ppm}$ \\
\hline$Z r^{*}$ & $\mathrm{HF}-\mathrm{HNO}_{3}-\mathrm{HClO}_{4}$ acid digestion \& $\mathrm{HCl}$ leach & ICP-MS & $0.5 \mathrm{ppm}$ & $500 \mathrm{ppm}$ \\
\hline
\end{tabular}

